\title{
SELF-REFERENCE ELICITS SLOWER RECALL FOR PERSONALITY \\ CONGRUENT ADJECTIVES: AN ITEM-SPECIFIC PROCESSING EFFECT
}

\author{
by
}

Glen Howell

A thesis submitted to the Faculty of Graduate and Postdoctoral Affairs in partial fulfillment of the requirements for the degree of

\author{
Doctor of Philosophy \\ in \\ Psychology \\ Carleton University \\ Ottawa, Ontario \\ (C) 2015
}

Glen Howell 


\begin{abstract}
The fact that people remember more personality trait adjectives in a memory test after making self-reference judgments, relative to other types of judgments (e.g., otherreference and semantics), has been attributed to greater elaborative (i.e., item-specific) and organizational (i.e., relational) processing. The present research asked whether personality self-concept renders more item-specific processing when the self-referenced materials are personality congruent. Previous attempts to address this question were equivocal, as brief recall tests were used and amount recalled was the indicator of itemspecific processing. Research shows that item-specific processing produces slower recall than does relational processing, whereas the latter protects against item loss on a second recall test. The present studies therefore tracked recall across two consecutive tests. Results demonstrated an uncrossed double dissociation in self-reference recall speed, wherein self-reported orderliness was associated with slower recall only when the adjectives described orderliness (Study 1; $\mathrm{N}=98$ ), and self-reported openness was associated with slower recall only when the adjectives described openness (Study 2; $\mathrm{N}=$ 92). In Study $3(\mathrm{~N}=163)$, participants who viewed their friend as highly orderly recalled orderliness adjectives encoded in an other-reference task more slowly, indicating that tapping the self-concept was not necessary to observe the association. However, the openness-recall speed relationship of Study 2 failed to replicate. In all studies, item loss was not related to personality or recall speed, suggesting relational processing differences were not responsible for the findings. Likewise, demand characteristics were likely not responsible either because personality measures in Study 2 were taken during mass testing. Recall speed was not associated with an indirect personality measure (i.e.,
\end{abstract}


Implicit Association Test), suggesting item-specific information was propositional, not associative. Theoretical considerations were centered on whether item-specific information rendered multiple memory traces, which is consistent with the slowing of recall predicted by a random search model of memory and cue-overload theory. Overall, convergent validity was fairly weak and predictive validity was poor, but methodological issues were identified and statistical refinements were suggested. The present studies nevertheless successfully linked individual differences in personality as represented in memory to the item-specific processing that occurred during self- and other-reference encoding. 


\section{Acknowledgements}

An undertaking such as putting together a dissertation is never a solo effort. I have been helped in numerous ways over the years from friends, family, and great staff at Carleton University. I hold a debt of gratitude to my supervisor, John Zelenski, for his unwavering encouragement, open-mindedness, timely and insightful feedback, and all the interesting conversations we had around research. The contributions and encouragement of my committee members, Johanna Peetz and Craig Leth-Steensen, have not gone unnoticed. Thank you for joining me on this ride and for your thought-provoking feedback and discussion around the research. A thank you as well to Daniel Burns for providing expertise and time in evaluating the work presented here as external examiner. Andrew Brook deserves thanks, not once, but twice, for providing expertise and time as internalexternal examiner for both my MA and $\mathrm{PhD}$ defence. It has been an honour to have such a great supervisor and committee.

On a less academic front, my wife, Robyn Howell, has been an amazing support over the years and I could not have succeeded without her. Likewise, my parents (Janet and David Howell), brothers (Graham and John Howell), and sister (Jennifer Edgar) have been especially supportive during the difficult times in graduate school. Similarly, Deanna Whalen and Jordan Schoenherr deserve special note for talking things through with me during challenging transitions (Deanna Messervey, Larry Grandmaison, and colleagues from National Defence as well). Thank you Etelle Bourassa for being incredibly kind and supportive. A final thank you to my fellow Happy Lab mates for making graduate school extra fun and memorable: Raelyne Dopko, Karin Sobocko, Steve Jones, Colin Capaldi, Stephanie Pineau, Zack Van Allen, among others. 


\section{Table of Contents}

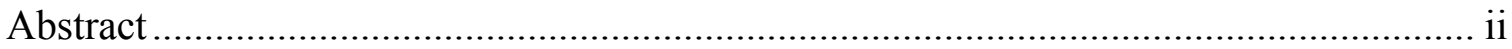

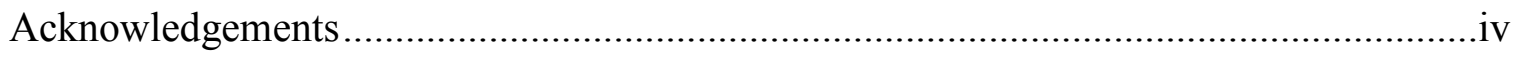

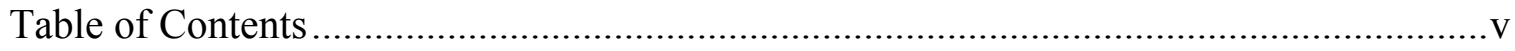

List of Tables .................................................................................................... vii

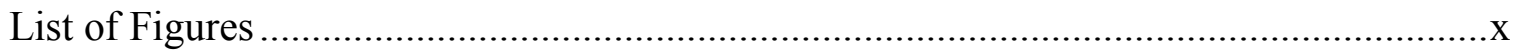

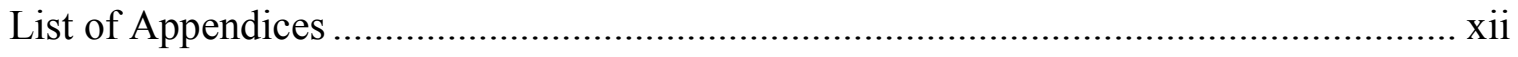

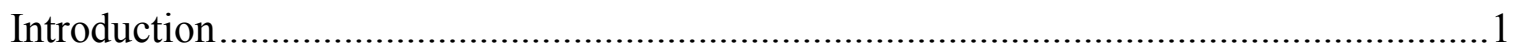

Levels of Processing and Organization in Memory ................................................... 10

The Self-Reference Effect................................................................................. 17

Measures of Elaborative and Organizational Processing.........................................22

Does Self-Reference Recall Performance Tell Us Something About Personality?...........30

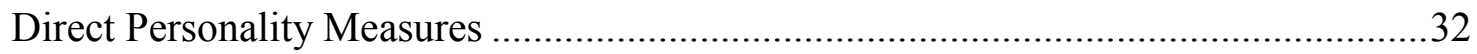

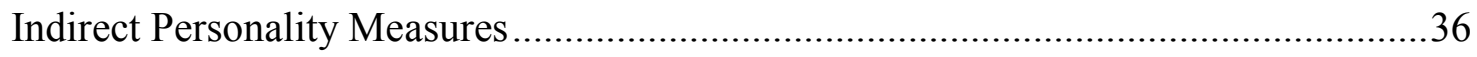

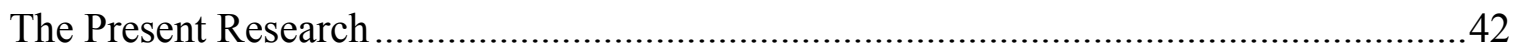

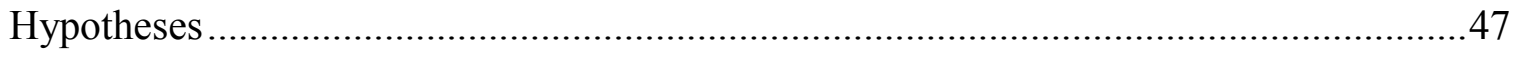

Self- and Other Reference Effects ......................................................................4

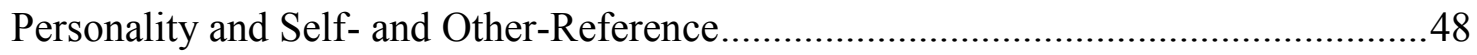

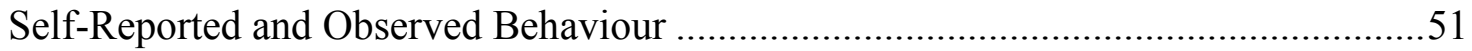

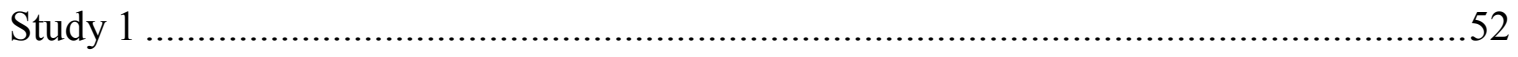

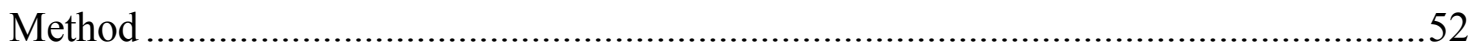

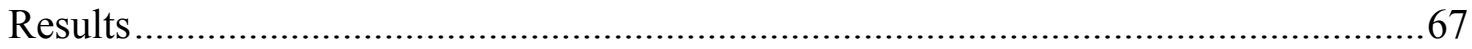




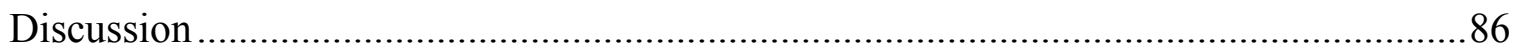

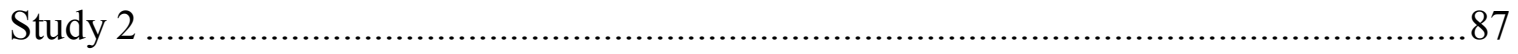

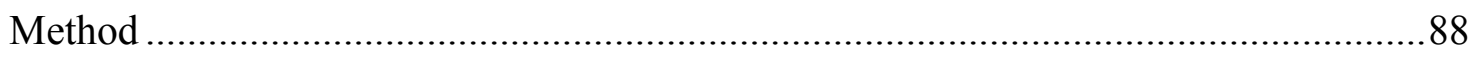

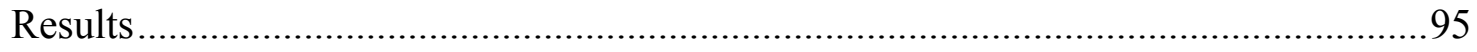

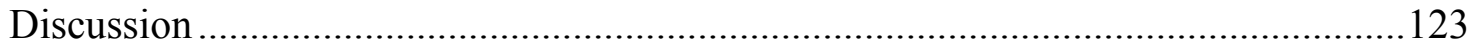

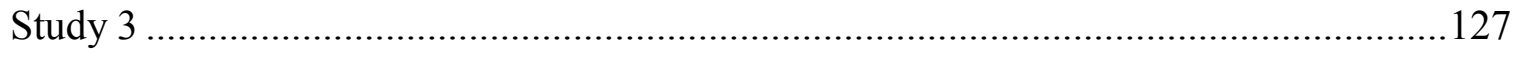

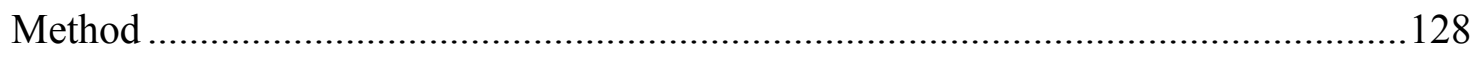

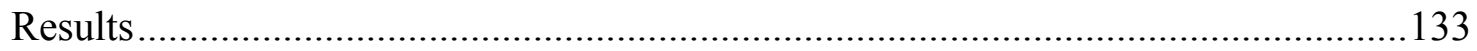

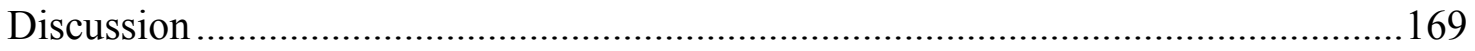

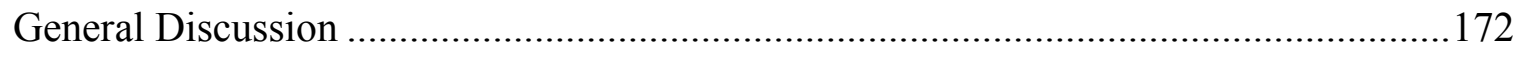

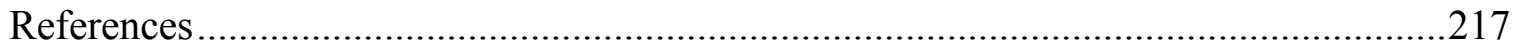

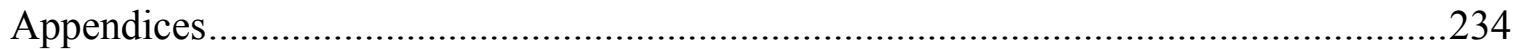




\section{List of Tables}

Table 1 Levels-of-Processing Items for the Orderliness and Impulse Control Facets of

Conscientiousness

Table 2 Mean Psycholinguistic Properties for the Orderliness and Impulse Control

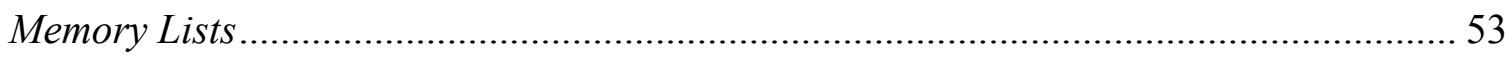

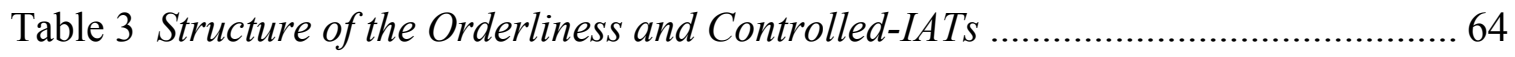

Table 4 Partial Correlations Among Direct and Indirect Measures of Conscientiousness

and Self-Reference Memory Processes in Study 1 ................................................... 76

Table 5 Zero-Order Correlations Between Direct and Indirect Personality Measures and

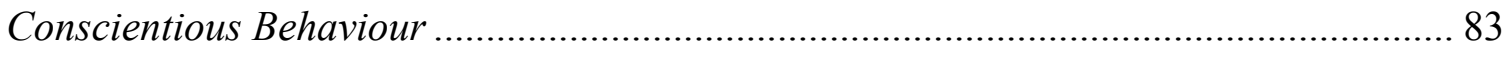

Table 6 Levels-of-Processing Items for the Imaginativeness and Orderliness Facets .... 89

Table 7 Mean Psycholinguistic Properties for the Imaginativeness and Orderliness

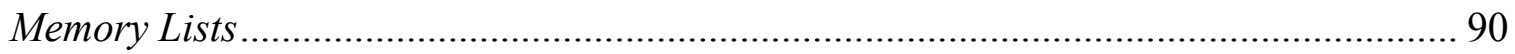

Table 8 Zero-Order Correlations Among Direct and Indirect Personality Measures and

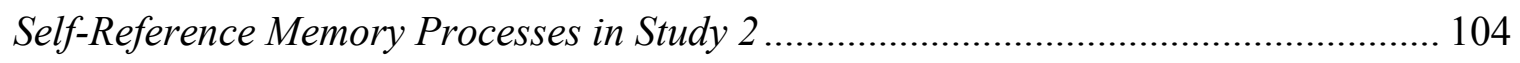

Table 9 Zero-Order Correlations Among Direct and Indirect Personality Measures and

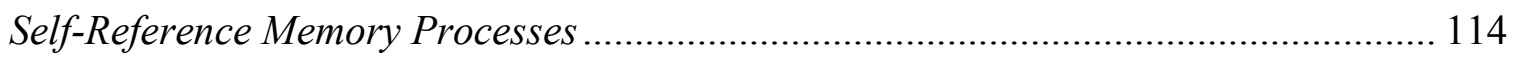

Table 10 Zero-Order Correlations Between Direct and Indirect Personality Measures

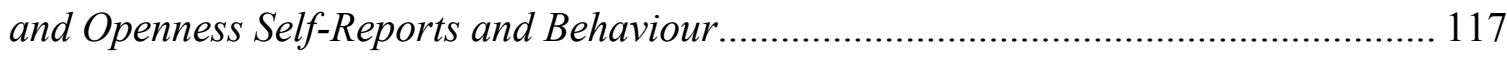

Table 11 Means and Standard Deviations of the Direct and Indirect Personality

Measures as a Function of Openness Behaviour in Study 2..................................... 119 
Table 12 Means and Standard Deviations of the Direct and Indirect Personality Measures as a Function of Self-Reported Openness Attitudes and Behaviours in Study 2

Table 13 Levels-of-Processing Items for the Trust, Imaginativeness, and Orderliness Facets.

Table 14 Mean Psycholinguistic Properties for the Trust, Imaginativeness, and

Orderliness Memory Lists.

Table 15 Zero-Order Correlations Among Direct and Indirect Measures of Openness and Self-Reference Memory Processes in Study 3 143

Table 16 Zero-Order Correlations Among Conscientiousness and Orderliness and SelfReference Measures of Openness

Table 17 Zero-Order Correlations Among the Direct and Self-Reference Measures of

Openness as a Function of Friend's Level of Orderliness ........................... 150 Table 18 Zero-Order Correlations Between Direct, Indirect, and Self-Reference Memory Measures and Self-Reported Openness Attitudes

Table 19 Means and Standard Deviations of the Direct, Indirect, and Self-Reference Memory Measures as a Function of Self-Reported Openness Attitudes and Behaviours 157 Table 20 Zero-Order Correlations Among Direct Measures of Orderliness and OtherReference Memory Processes in Study 3

Table 21 Zero-Order Correlations Among Participants' Best Friend's Openness and Imaginativeness and Direct, Indirect, and Other-Reference Measures of Orderliness 163 Table 22 Zero-Order Correlations Among the Direct, Indirect, and Other-Reference Measures of Orderliness as a Function of Participants' Level of Openness 165 
Table 23 Zero-Order Correlations Between Direct and Other-Reference Measures of Orderliness and Other-Reported Orderliness Attitudes ............................................ 168 


\section{List of Figures}

Figure 1. Cumulative recall as a function of various combinations of item-specific and relational processing (adapted from Burns \& Schoff, 1998) ...................................... 25

Figure 2. Cumulative recall in Study 1 as a function of encoding task........................ 70

Figure 3. Cumulative recall in Study 1 as a function of orderliness, presented separately

for the self-reference and combined semantic, rhyming, and structural tasks ................ 81

Figure 4. Cumulative recall in Study 2 as a function of encoding task........................ 98

Figure 5. Cumulative recall in Study 2 as a function of openness presented separately for the self-reference and combined semantic, rhyming, and structural tasks. 108 Figure 6. Cumulative recall in Study 1 as a function of openness presented separately for the self-reference task and the combined semantic, rhyming, and structural tasks. ....... 110 Figure 7. Cumulative recall in Study 2 as a function of imaginativeness presented separately for the self-reference and combined semantic, rhyming, and structural tasks 113 Figure 8. Cumulative recall in Study 2 as a function of orderliness presented separately for the self-reference and combined semantic, rhyming, and structural tasks.............. 116 Figure 9. Cumulative recall in Study 3 as a function of encoding task...................... 138 Figure 10. Cumulative recall in Study 3 as a function of openness presented separately for the self-reference and semantic tasks. 147 Figure 11. Cumulative recall in Study 3 as a function of openness at the medium level of best friend's orderliness presented separately for the self-reference and semantic tasks 153 Figure 12. Cumulative recall in Study 3 as a function of orderliness presented separately for the other-reference and semantic task 162 
Figure 13. Cumulative recall in Study 3 as a function of orderliness for the medium openness group presented separately for the other-reference and semantic tasks .......... 167 Figure 14. A social knowledge structure depicting the self-concept as a collection of associations (connections) between the "ME" node and traits (e.g., "intelligent"). Copied from Greenwald et al. (2002) for illustrative purposes................................................. 196 


\section{List of Appendices}

Appendix A: Procedure for Creating the Levels-of-Processing Lists ...........................234

Appendix B: Rhyming and Semantic Orienting Questions ....................................237

Appendix C: Reading Span Stimuli and Order of Presentation..................................238

Appendix D: Procedure for Creating Recognition Memory Lists ................................241

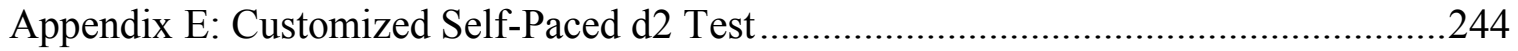

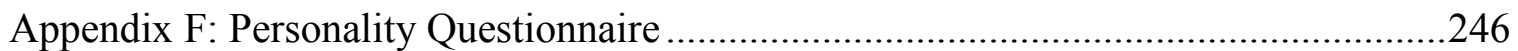

Appendix G: Behaviour Report Questionnaire ..................................................249

Appendix H: Presentation Order for the Levels-of-Processing Task ..........................252

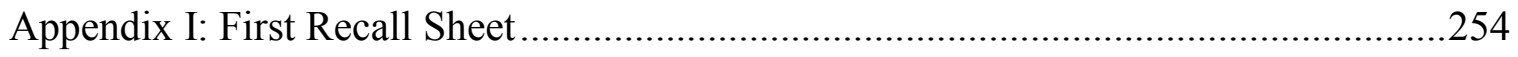

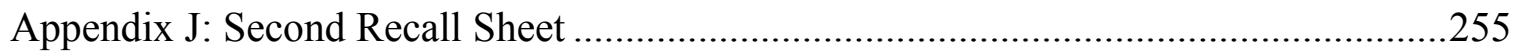

Appendix K: Zero-Order Correlations Among the Memory-Based, Direct, and Indirect

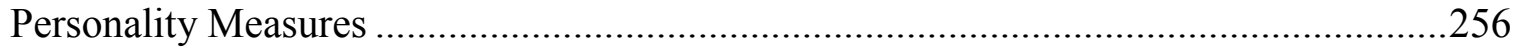

Appendix L: Memory List and Recognition Items for Study 2. ................................257

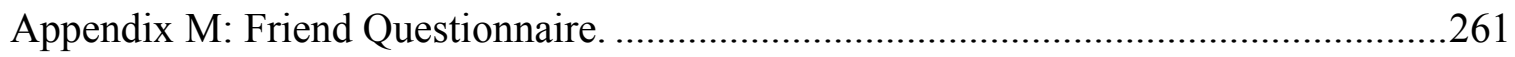

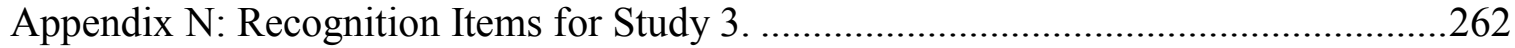




\section{Introduction}

Experimental psychologists have long been interested in understanding what constitutes the self (James, 1890). Researchers within various sub-disciplines of psychology have adopted different approaches in pursuit of this goal. In the lexical approach to studying personality traits, researchers have repeatedly found that a small number of broad traits comprise the various dimensions of disposition that are relevant to the self (e.g., Goldberg, 1990; Lee \& Ashton, 2004; Norman, 1963). One particularly well-studied taxonomy postulates five broad traits that are each composed of narrower facets (i.e., the Big Five: extraversion, neuroticism, conscientiousness, agreeableness, and openness to experience; McCrea \& Costa, 1987). In another sub-discipline, social cognitive researchers have made a distinction between the self-report method of personality assessment traditionally used in the lexical approach (a direct measure) and performance on a cognitive task designed to measure the degree of association between the mental representations of the self (self-concept) and traits (an indirect measure; e.g., Implicit Association Test, IAT; Greenwald, McGhee, \& Schwartz, 1998; see Schnabel \& Asendorpf, 2010 for a review). They found support for the idea that both types of measures are important for a complete understanding of how personality traits can explain various behaviours. In yet another sub-discipline, memory researchers have shown that engaging the self-concept in a cognitive task can have strong effects on information processing, including free recall and recognition (e.g., Symons \& Johnson, 1997). The present research aimed to link the three approaches noted above in a novel way. Specifically, it focused on linking direct and indirect personality trait measures and trait related behaviours with the basic memory processes that occur while engaging the 
self-concept in a cognitive task. In doing so, it had the potential to produce a new personality assessment tool that is rooted in memory research.

In a classic memory study that engages the self-concept, Rogers, Kuiper, and Kirker (1977; Rogers, 1977) found that judging whether personality trait adjectives relate to one's self (as one would do on a personality self-report; e.g., Goldberg, 1990) yields better free recall in a surprise memory test and better recognition than making any other type of judgment (e.g., rhyming or semantic judgments). They argued that this memorial benefit, known as the self-reference effect (SRE), stems from relating the words to the self-concept, which generates an elaborate memory trace upon encoding (elaborative processing). Memory researchers have argued that elaborative processing provides itemspecific information (i.e., idiosyncratic retrieval cues) that support recall and recognition of particular items that have been encoded (Burns, 1993; Burns \& Hebert, 2005; Einstein \& Hunt, 1980; Hunt \& Einstein, 1981; Hunt \& McDaniel, 1993; McCabe et al., 2004; see also Craik \& Tulving, 1975). In contrast to the elaborative processing hypothesis of the SRE, Klein and Kihlstrom (1986) argued that it might be due solely to the fact that judging words for self-descriptiveness provides a means to organize the list of words into categories (organizational processing; e.g., words that describe me vs. those that do not), something that other comparison encoding tasks have not encouraged. Memory researchers (e.g., Raajimakers \& Shiffrin, 1981; Slamecka, 1968) propose that organizational processing provides a small set of categories that narrow the search of memory during free recall and can act as a retrieval plan. In a similar vein, Einstein and Hunt (1980; Hunt \& Einstein, 1981; Hunt \& McDaniel, 1993) suggest that organizational processing exploits the common characteristics of the encoded words to provide 
relational information that is available in semantic memory (e.g., a common category label). In a clever set of studies, Klein and Loftus (1988) showed that both elaborative and organizational processing are important factors in explaining the SRE, a conclusion that was supported in a meta-analytic review (Symons \& Johnson, 1997). However, although elaborative processing has much support as a causal factor in producing the SRE, research supporting that conclusion has not used a suitable measure of elaborative processing, which is an issue that was addressed in the present thesis.

Specifically, researchers have argued that appealing to a measure of elaborative processing that depends on amount recalled to explain the SRE renders a circular causeand-effect relationship. Specifically, because the SRE is evidenced by a greater amount recalled, one cannot propose that a construct measured by amount recalled (e.g., elaboration) causes the SRE (Baddeley, 1978; Eysenck, 1978; Klein, Loftus, \& Schell, 1994). As an example, if researchers want to prove that extraverted behaviour causes positive emotions, they cannot operationally define a measure of extraverted behaviour that is based on positive emotions. If they did, they would essentially be making the unhelpful claim that positive emotions cause positive emotions. However, if some other measure of extraverted behaviour that is independent from positive emotions was devised, such as the likelihood of talking with a stranger in a given context, they could rightfully claim that extraverted behaviour operationally defined in that way causes positive emotions, so long as likelihood of talking with a stranger has been manipulated (e.g., in an act introverted versus act extraverted manipulation; Zelenski, Santoro, \& Whelan, 2011) and measures of positive emotions increase when participants act extraverted. 
Fortunately, measures of elaborative and organizational processing that are largely independent of amount recalled have been devised. Specifically, in what is known as a repeated-testing paradigm (Burns, 1993; Klein, Loftus, Kihlstrom, \& Aserson, 1989), participants are asked to recall previously encoded items in two subsequent memory tests. Items that are remembered on the second test but not the first (item gains) are suggestive of elaborative processing, in so far as the item-specific information made available from elaborated encodings take sufficient time to render successful retrieval of list items, which may not occur until the second recall test. In contrast, items that are recalled on the first test and are forgotten on the second (item losses) are suggestive of a lack of organizational processing. In particular, the relational information acquired during encoding (i.e., a category that organizes the encoded items) or a retrieval plan protect against item losses. Klein et al. (1994) found that self-reference encoding rendered fewer item losses than other encoding tasks, and marginally more item gains, supporting the previous conclusion that elaborative and organizational processing are key factors in explaining the SRE using a measure that was largely independent from amount recalled.

However, a criticism has been levelled against the item gain measure of elaborative processing. In particular, Burns and Hebert (2005; see also Burns \& Schoff, 1998; Wixted \& Rohrer, 1994) have shown that item gains depend on the amount of time allowed for recall in each memory test. For instance, with two 5-minute tests, there may be significant item gains. However, all other things being equal, with two 10 -minute tests, all items recalled in minutes 6 through 10 would be recalled on the first test, leaving few items to-be-gained on the second test. The authors proposed examining cumulative recall curves to circumvent the problem (Bousfield \& Sedgewick, 1944; see Wixted \& 
Rohrer, 1994 for an excellent review). In a plot of the amount of unique items recalled as a function of time (e.g., in minutes), the best fitting curve, or cumulative recall curve, is described by two parameters: the rate of approach to asymptote and the asymptote. The rate of approach to asymptote in particular provides insight into the amount of elaborative processing engaged during encoding. More precisely, shallow recall curves (low rate of approach) generally indicate that item gains happened more slowly throughout the recall task, which suggests more item-specific information was encoded. In contrast, steep recall curves (high rate of approach) generally indicate that most recalled words were produced early in the recall task, which suggests more relational information was encoded by organizational processes. The relational information provides a few common characteristics of the encoded words (e.g., categories) that can dramatically constrain the search of memory (e.g., Raajimakers \& Shiffrin, 1981) rendering faster recall.

Under certain conditions when a high amount of item-specific and relational information has been encoded, the cumulative recall curve can appear steep initially, reflecting the effective deployment of relational information, but can also appear shallow later in the recall task, reflecting the continued successful retrieval of new items from item-specific information (Burns \& Schoff, 1998). The estimate of rate of approach in this case tends to be high, suggesting relatively fast recall overall, despite the contribution of item-specific information. Given the complex interplay between item-specific and relational information and how it impacts the time course of free recall, Burns and Hebert (2005; Burns \& Schoff, 1998) suggested examining a plot of cumulative recall in addition to the rate of approach to asymptote to better understand the time course of recall. Analysis of cumulative recall curves has been fruitful in research on false memory 
(Burns, Jenkins, \& Dean, 2007; Burns et al., 2006), proactive interference (Burns, 1989; Wixted \& Rohrer, 1993), free recall (Burns \& Hebert, 2005; Burns \& Schoff, 1998), and the survival processing effect (Burns, Hwang, \& Burns, 2011). Within the literature on the SRE, however, the use of cumulative recall curves, as a way to measure elaborative and organizational processing, has not been systematically examined. Thus, the present research took a cumulative recall approach to examine the relative contributions of elaborative and organizational processing on the recall advantage of the SRE, using measures that were largely independent of amount recalled.

Despite the fact that personality trait adjectives are typically used as materials in studies on the SRE (Symons \& Johnson, 1997), few studies have examined whether free recall and recognition of trait adjectives relates to a structured aspect of self-concept (i.e., individual differences). Markus (1977) suggested that personality dimensions may be mentally represented in self-schemata (i.e., a structured cognitive representation of the self), and self-schemata influence information processing (see also Bower \& Gilligan, 1979; Rogers et al., 1977). Most studies on the SRE have selected from a broad range of trait adjectives without examining performance as a function of specific personality trait dimensions. A few exceptions, however, are notable. Sullivan (1998) compared selfreport measures of the Big Five dimensions to recall and recognition performance following a self-reference task on trait adjectives associated with the Big Five. The results showed that personality self-reports were not related to the amount recalled of the respective trait adjectives and were largely unrelated to subsequent recognition. In contrast, Katz (1987) found that individuals who scored high on a remote association test, which is indicative of high creativity (a facet of openness to experience), recalled more 
adjectives describing creativity than adjectives describing other traits relative to those who scored low on the remote association test. Moreover, the difference in amount recalled only occurred following self-reference encoding (i.e., not semantic or structural encoding).

The latter study is supportive of the main tenet of the present thesis, but the former study is not supportive. Amount recalled, however, is not a viable measure of elaborative processing when the to-be-explained effect is the amount recalled. A plausible explanation for the better recall is that highly creative individuals were better able to organize the adjectives describing creativity during self-reference or that they elaborated more on those adjectives during encoding, or both. Moreover, it is unlikely that participants were given enough time for recall to reach an approximation of their asymptotic level. In Katz's (1987; Experiment 2) research, participants encoded 118 trait adjectives and were only given 5 minutes for recall. In Sullivan's (1998) research, participants encoded 120 trait adjectives and were given a maximum of 10-minutes for recall. Roediger and Thorpe (1978; see also Wixted \& Rohrer, 1994) have shown that recall can persist well beyond 5 or even 10 minutes. Burns (1993) suggested using a 15 to 20 minute recall period in order to allow item-specific information to be fully exhausted. When amount recalled is assessed at only one time point (i.e., the end of a 5-minute test), erroneous conclusions may be made about recall differences between experimental conditions, unless enough time to reach an approximation of asymptotic recall is provided (especially when the rate of approach to asymptote differs greatly between conditions). Hence, given the limitations of previous research, it is not clear whether elaborative processing of trait adjectives during self-reference encoding relates to 
personality as represented in the self-concept. The main goal of the present research was therefore to examine whether individual differences on a personality trait are related to variation in cumulative recall following self-reference encoding of respective trait adjectives.

In terms of personality assessment, social cognitive researchers have made a distinction between self-report (a direct measure) and performance on a cognitive task (an indirect measure). Following the development of indirect measures of attitude and evaluation (e.g. Implicit Association Test; IATs; Greenwald, McGhee, \& Schwartz, 1998), researchers sought to construct and validate IATs that measure the Big Five personality traits (Grumm \& Collani, 2007; Schmukle, Back, \& Egloff, 2008). In line with the common interpretation of IAT effects (Hofmann, Gawronski, Gschwendner, Le, \& Schmitt, 2005), they suggested that Big Five IATs measure the relative strength of associations in memory between the self (vs. others) and a particular pole of a trait (e.g., conscientiousness) relative to the other pole (e.g., undirectedness). Researchers have found that Big Five IATs have moderate test-retest reliability, satisfactory internal consistency, poor to moderate convergent validity, and fair divergent validity.

Additionally, research has shown that Big Five IATs predict relevant spontaneous behaviours (Steffens \& Schulze-König, 2006) and have incremental validity over direct measures of personality in predicting trait related behaviours (Schmukle, Back, \& Egloff, 2008; Back, Schmukle, \& Egloff, 2009). Furthermore, Egloff and Schmukle (2002) validated an anxiety IAT and showed it had incremental validity over a direct measure of trait anxiety in predicting anxiety related behaviours elicited by perceived task failure and public speaking. In addition, Asendorpf, Banse, and Mucke (2002) found that a shyness 
IAT, but not a self-report measure, predicted shyness behaviours that are difficult to control while self-reported shyness, but not the shyness IAT, predicted shyness behaviours that are more easily controlled (i.e., a double dissociation). Thus, beyond establishing the predictive validity of IATs as indirect measures of personality selfconcept, these studies suggest that personality IATs are able to predict spontaneous inthe-moment behaviours that are not captured by a direct measure of personality traits.

Research on personality self-concept therefore suggested that the present studies obtain validated direct and indirect personality measures. It may be possible that the hypothesized elaborative processing measures of personality self-concept are more related to the strength of associations in memory between the self and trait representations (i.e., IAT effects) than to the outcome of propositional reasoning that has been proposed as the source of information considered in a direct personality measure (Schnabel \& Asendorpf, 2010; see also Gawronski \& Bodenhausen, 2006, 2011). Furthermore, behavioural indicators of personality traits were included to evaluate the predictive validity of the memory-based personality measures.

In sum, the main tenet of my thesis is that individual differences in personality traits should be related to individual differences in the rate of approach to asymptote for cumulative recall curves after self-reference encoding of corresponding personality trait adjectives. In three studies, personality was assessed directly (i.e., self-reports) and indirectly (i.e., IATs). Trait relevant behaviour was measured to assess predictive validity. I hypothesized that people who are high on a personality trait would exhibit greater elaborative processing of corresponding trait adjectives, as evidenced by shallower cumulative recall curves, relative to those who are lower on that trait. 
Moreover, in a test of divergent validity, I hypothesized that self-reference judgments would be key for these relationships to hold. That is, other encoding tasks (e.g., semantic or phonetic) were not expected to render such patterns of data. Finally, if the rate of approach to asymptote indeed captures differences in personality self-concept, I hypothesized that it would predict trait relevant behaviour. Three studies addressed these hypotheses to provide strong evidence that personality self-concept is tapped by elaborative processing when making a self-reference judgment, a point that was first made but not tested in the first demonstration of the SRE (Rogers et al., 1977; see also Bower \& Gilligan, 1979 and Markus, 1977).

Moving forward, the levels-of-processing and organization approaches to memory research are reviewed in more depth, followed by the literature on personality trait assessment, including direct and indirect measures. Then, the present studies are outlined followed by statements of specific hypotheses.

\section{Levels of Processing and Organization in Memory}

Early memory research can be divided into separate paradigms. Craik and Lockheart's (1972) levels-of-processing framework emphasized the importance of the type of judgment people make about stimuli for subsequent memory performance. They proposed that perception of stimuli initiates a low level of processing (e.g., physical aspects of the stimuli) and depending on factors such as task demands, the nature of the stimuli, and available processing capacity, deeper levels of processing (e.g., phonetics or semantics) may result. For instance, if a task requires one to determine whether words contain two or more of the letter "e", a low level of perceptual analysis is sufficient to complete the task, and deeper processing into the semantics of the words is not likely to 
occur. In contrast, when the task requires one to determine some aspect of the words' semantics (e.g., a category or synonym judgment), the processing must proceed beyond a perceptual analysis to a deeper level where semantic information is available. The level of processing achieved determines the durability of the memory trace, with deeper levels providing better memory. Furthermore, when a semantic level is achieved, learned cognitive structures become part of the memory trace. Hence, when participants make a judgment about the meaning of a word (semantic encoding), the levels-of-processing framework suggests superior memory performance should result relative to making a judgment about the word's phonetics (phonetic encoding) or graphemes (structural encoding).

In a series of ten experiments, Craik and Tulving (1975) tested the idea that deeper processing provides superior memory by manipulating the type of judgment (orienting task) participants were required to make when viewing stimuli. The results clearly showed that semantic encoding provides superior free recall and recognition than phonetic or structural encoding. It was originally suggested that the amount of time to accomplish the orienting task could provide a measure of depth-of-processing that is independent from the to-be-explained phenomenon (i.e., the greater amount recalled in deeper processing conditions). Furthermore, the levels-of-processing framework suggested that as processing proceeds through discrete levels from shallow to deep, response times should be linearly related to depth and hence memory performance. Although semantic tasks required more time to accomplish than shallower tasks, further results showed that the amount of time to accomplish the processing task could not account for the memorial benefit provided by deep processing. Specifically, a simple 
semantic task that took less time to accomplish relative to a complex structural task still rendered better memory. Based on this finding, the authors suggested revisions to the levels-of-processing framework.

Specifically, Craik and Tulving (1975) suggested the mechanism responsible for the levels-of-processing effect is cognitive elaboration, which could be structural, phonetic, or semantic. Elaborative processing need not occur in discreet stages. It was suggested that semantic tasks yield more elaborate memory traces than shallower tasks, and in turn more retrieval cues for successful free recall and recognition. However, Baddeley (1978) and Eysenck (1978) were quick to point out the circularity in the causeand-effect relationship between depth-of-processing (or cognitive elaboration) and superior recall. In particular, the levels-of-processing effect is evidenced by a higher amount recalled in semantic than phonetic or structural tasks, and the cause, elaborative processing is also evidenced by the amount recalled. Measures of elaborative processing that are largely independent of amount recalled are discussed in an upcoming section. In contrast to the levels-of-processing paradigm, much research points to the importance of organization for memory (Bower, 1970; Mandler, 1967). Research on organizational processing suggests memory performance can be greatly impacted by the semantic associations among the presented words. That is, when words belong to the same semantic category, people may come to associate the encoded words with a higherorder category (e.g., people may associate punctual, efficient, and prompt with the more general category conscientiousness). Research also suggests that organizational processing can generate a hierarchical retrieval scheme that participants may use to narrow the search of memory when asked to recall previously processed words (Bower, 
Clark, Lesgold, \& Winzenz, 1969; Raajimakers \& Shiffrin, 1981; Slamecka, 1968). The amount of organizational processing engaged has traditionally been measured mathematically by the degree to which members of the same category are listed together during free recall (category clustering, e.g., Bousfield \& Bousfield, 1966; Roenker, Thompson, \& Brown, 1971).

Hyde and Jenkins (1969) examined recall performance under conditions where participants were informed about the memory test (intentional learning) and when they were not informed (incidental learning). Prior to recall, participants engaged in one of three different encoding tasks: semantic, structural, or a control condition that did not require making a judgment. They were presented a list of words that contained highly associated word pairs. Of particular interest was the amount of organization evidenced in the recalled words and impact of intentional versus incidental learning on recall. Organization was evidenced by the presence of both members of the highly associated word pairs listed in order. The results across three studies showed that shallower encoding tasks yielded worse recall than control and semantic encoding under both intentional and incidental encoding instructions (i.e., a levels-of-processing effect in intentional and incidental learning). Furthermore, there was greater organization in people's recall when they encoded items semantically or in the control condition relative to the shallower structural encoding tasks.

Hyde and Jenkins (1969) argued that the various encoding tasks affect the amount of organization in recall as well as the total amount recalled, whether participants know they will be tested or not. In line with Tulving's $(1962,1966)$ idea, the data supported the notion that organization during storage (whether covert or not) may be responsible for the 
encoding task differences in organized recall. Specifically, when items are encoded semantically, the individual traces for associated words activate common structures in semantic memory (e.g., a category). During recall, the reactivation of the structures (categories) by memory trace information would then make readily available the word associate for immediate recall (hence, organized recall). When the task is structural, however, the words are likely only processed at a grapheme level (i.e., groups of letters), where the individual traces for associated word pairs would likely only activate a common grapheme category (e.g., words that contain the letter "e"). During recall, the reactivation of the grapheme category would not be able to facilitate retrieval of the specific word associates that were processed. Hence, the orienting task performed on stimuli effects the use of organizational processing for recall. The study is a classic example of how organizational processing makes use of semantic memory whereby associations among the presented words can be exploited, whether by a common category (Bower, 1970) or retrieval scheme (Bower et al., 1969).

Einstein and Hunt (1980; Hunt \& Einstein, 1981) presented a set of elegant experiments that brought together research on levels-of-processing (Craik \& Lockheart, 1972; Craik \& Tulving, 1975) and organization in memory (e.g., Bower, 1970). They showed that elaborative and organizational processing have an additive effect on recall and recognition, which provided strong evidence that these processes are qualitatively different. In the first experiment, they crossed three orienting tasks common to the levelsof-processing framework (semantic: pleasantness rating; non-semantic: rhyme rating; or none) with three orienting tasks common to research on organizational processing (semantic: taxonomic category-sorting, non-semantic: first letter sorting; or none) in a 
between-subjects design. During encoding, participants completed up to two orienting tasks. For instance, participants assigned to the pleasantness rating and taxonomic category-sorting condition (pleasantness-taxonomic condition) performed a semantic elaborative and semantic organizational processing task on all words. That is, they rated the words for pleasantness and sorted the words into taxonomic categories, both of which involve semantic memory. The memory list was composed of 36 concrete nouns taken as six exemplars from six taxonomic categories (e.g., fruit, animals, kitchen utensils, etc.), under the constraint that the six nouns belonging to each category started with an $1, \mathrm{c}, \mathrm{s}$, $\mathrm{b}, \mathrm{m}$, or $\mathrm{t}$, so the words could be sorted by first letter by those assigned the non-semantic organizational task.

Einstein and Hunt (1980) found that the pleasantness-taxonomic condition provided the best recall and recognition out of all nine conditions. Furthermore, the semantic tasks promoting either elaborative (pleasantness rating) or organizational (taxonomic category-sorting) processing yielded higher recall and better recognition than their non-semantic counterparts (i.e., than the rhyme rating and first letter sorting tasks; the typical levels-of-processing effect). Moreover, semantic organizational processing (taxonomic category-sorting) produced higher category clustering in free recall than did semantic elaborative processing (pleasantness rating). In sum, the data supported the conclusion that elaborative and organizational processing have an additive effect on recall and recognition, and are supported by different mechanisms (based on systematic differences in recall, recognition, and category clustering). In general, the authors proposed that elaborative processing yields item-specific information that can be useful for distinctiveness in memory (high correct recognition, a low rate of false alarms, and 
low category clustering) and organizational processing yields relational information that organizes the list items by a higher-order category and may be used for a retrieval scheme (lower correct recognition, more false alarms, but higher category clustering). In this way, the two types of processing and the information activated in memory independently contribute to recall in a cooperative manner.

Einstein and Hunt's (1980) first experiment used an organized list of items (i.e., six exemplars from six different taxonomic categories) that participants would likely have viewed as having a categorical structure (e.g., fruit, animals, kitchen utensils, etc.). The authors hypothesized that a well-structured list (a related list) would benefit less from organizational processing than it would from elaborative processing, because the obvious category structure provides relational information that is redundant to that provided by an organizational processing task. Likewise, a list of words from diverse categories without an obvious structure (an unrelated list) would benefit more from organizational than from elaborative processing, because the disparate words would encourage acquisition of individual-item information making an elaborative processing task relatively redundant. Hence, they predicted that word list structure would interact with the encoding task (elaborative vs. organizational) in free recall and recognition performance. To explore this hypothesis, the authors conducted a second experiment where they crossed word list structure (unrelated and related lists) with type of semantic processing (pleasantness or taxonomic category-sorting) in a between-subjects design.

As predicted, Einstein and Hunt (1980) found that elaborative processing (i.e., pleasantness ratings) yielded higher recall for a related word list $(M=19.58)$ than for an unrelated one $(M=15.75)$. Moreover, organizational processing (i.e., taxonomic 
category-sorting) yielded higher recall for the unrelated word list $(M=18.58)$ than for the related one $(M=16.17)$. In addition, the degree to which categorically related items were recalled together (i.e., category clustering) was negligible when participants engaged in elaborative processing on the unrelated word list (because the task and word list only encouraged acquisition of individual-item information), while the three other conditions showed significant clustering but to varying degrees (because either the task or word list encouraged acquisition of relational information). Specifically, organizational processing on the related list showed the most clustering while elaborative processing on the related list and organizational processing on the unrelated list showed intermediate clustering. The authors argued that a categorical list structure promotes acquisition of relational information, supported by the finding that the organizational task and the related list each provided similar amounts of clustering. In sum, the authors put forward a new framework that integrated research on levels-of-processing and organization in memory. The implication is that the relative quantities of individual-item and relational information elicited by the combination of the encoding task and word list structure explains differential performance on recall and recognition. The framework also further describes how elaborative and organizational processing work together to provide the individual-item and relational information available in memory (see Hunt \& McDaniel, 1993 for an excellent review).

\section{The Self-Reference Effect}

Rogers et al.'s (1977) seminal research introduced and examined the impact of a self-reference encoding task on incidental free recall following the standard levels-ofprocessing manipulation. In the first experiment, participants judged a series of 40 
personality trait adjectives for self-reference ("Describes you?"), semantics (synonym judgment), phonetics (rhyming judgment), or structure ("Big letters?"). The authors demonstrated that people remember a higher proportion of personality trait adjectives after self-reference encoding $(M=.30)$ relative to semantic $(M=.13)$, phonetic $(M=.07)$, or structural $(M=.03)$ encoding. In the second experiment, they used different comparison encoding tasks thought to encourage more processing and tested participants in groups. They still found that self-reference $(M=.32)$ produced a higher proportion recalled than encoding semantics $(M=.19)$, phonetics $(M=.20)$, or structure $(M=.20)$. These findings were particularly striking because research on levels-of-processing had consistently shown that semantic encoding tasks produce the highest free recall performance. Rogers et al. argued that the observed SRE is due to associating the memory list items to the self-concept rendering a highly elaborate memory trace and effective set of retrieval cues (Craik \& Tulving, 1975). Very importantly, their research suggested that the levels-of-processing framework and self-reference encoding offer a useful methodology to examine cognitive biases associated with the mental representations of the self (i.e., self-concept) as manifested through memory performance (see also Markus, 1977).

In contrast to Rogers et al.'s (1977) elaborative processing explanation for the SRE, Klein and Kihlstrom (1986) explored whether it may be due to organizational processing. They argued that the self-reference task promotes organization of memory list items into salient self-related categories (e.g., describes me versus does not describe me), whereas the comparison encoding tasks that have been used in previous studies on the SRE have only promoted elaboration (e.g., pleasantness ratings, thinking of a definition, 
etc). In their fifth experiment, participants were presented 64 nouns that specified various occupations, half of which required a college education. Participants were assigned one of four conditions representing the factorial cross between encoding task (semantic or self-reference) and whether the task encouraged organization (unorganized or organized). In the tasks that encouraged organization, the presented nouns (e.g., surgeon) were preceded by the question "Does this job require a college education?" in the semantic task (i.e., semantic taxonomic sorting) and "Have you ever wanted to be a?" followed by the word surgeon in the self-reference task (i.e., organization by perceived career aspirations). In the tasks that did not encourage organization, the presented nouns were preceded by idiosyncratic questions (e.g., "Does this person perform operations?" followed by the word surgeon) in the semantic task and by idiosyncratic sentence frames (e.g., "I place complete trust in my XXXX?" followed by the word doctor) in the selfreference task. The focus in these later tasks was on processing the idiosyncratic meaning of each word (or the personal opinion of the participant) without encouraging organization of the lists. Klein and Kihlstrom (1986; Experiment 5) found that selfreference encoding did not produce better recall than did semantic encoding $(M=.52 \mathrm{vs}$. $M=.51)$. Moreover, a SRE could be observed when comparing the organized selfreference condition $(M=.55)$ to the unorganized semantic condition $(M=.46)$, supporting the authors' argument that previous studies have been comparing these two conditions where organization is a confounding variable. In sum, they concluded that organizational processing alone could account for the SRE.

However, in subsequent research, Klein and Loftus (1988) adopted the additive effects logic from Einstein and Hunt's (1980; Hunt \& Einstein, 1981) work and showed 
that both organizational and elaborative processing independently contribute to the SRE, depending on the categorical structure of the to-be-remembered items. When the word list encouraged organizational processing (i.e., many items from the same category), selfreference encoding provided a mnemonic advantage relative to encoding tasks that encouraged organization but not relative to tasks that encouraged elaboration. In contrast, when the word list encouraged elaborative processing (i.e., all items from different categories), self-reference encoding provided a mnemonic advantage over encoding tasks that encouraged elaboration, but not over encoding tasks that encouraged organization. In essence, both organizational and elaborative processing in combination account for the SRE, but the categorical structure of the word list can determine which process becomes more evidenced in the mnemonic advantage.

A meta-analytic review (Symons \& Johnson, 1997) supported the idea that elaborative and organizational processing contribute to the SRE. In particular, following the logic put forward by Einstein and Hunt (1980) and Klein and Loftus (1988), Symons and Johnson found that across all relevant studies $(n=129)$, the SRE was significant when the comparison task encouraged organizational or elaborative processing (and the size of these two SREs did not differ). More importantly, the SRE was significantly smaller when the comparison task evoked both organizational and elaborative processing (e.g., the other-referent task; "Describes your best friend?") relative to tasks that evoked only one type of processing, and it was still statistically significant. Based on these results, Symons and Johnson (1997) supported Klein and Loftus' dual-process explanation of the SRE, but speculated that the robust difference in recall between selfreference and semantic encoding may be due to the fact that the: 
typical SR [self-reference] task (a) uses trait domains as stimulus items more than $80 \%$ of the time, (b) taps trait domains likely to have been elaborated on many times using SR, (c) is likely to promote an SR mode in retrieval because of this practiced elaboration of traits, and (d) taps trait domains that are, because of the two points just mentioned (b and c) likely to be highly organized along selfrelated (or at least person-referent) dimensions (p. 387).

Symons and Johnson's (1997) speculation suggests it may be possible that personality trait information that is frequently accessed, highly elaborated on, and well organized in the self-concept may explain variation in the elaborative and organizational processing involved in the SRE. If individual differences in self-concept with respect to these personality trait dimensions explains variation in the encoding of item-specific or relational information that gives rise to the SRE, one would expect to find evidence of more elaborate processing during self-reference for those with a well-developed selfconcept with respect to the particular trait domain represented by the stimulus materials (e.g., trait conscientiousness adjectives) relative to those with a less well-developed selfconcept with respect to that trait dimension. For example, a highly conscientious individual may show evidence of greater elaborative processing in recall following selfreference encoding of conscientiousness adjectives than those who display average or below average conscientiousness. Suggestive of that possibility, when comparing selfreference to semantic encoding tasks, Symons and Johnson found that trait adjectives yielded a larger SRE than nouns (i.e., personality adjectives are more self-relevant in general than are nouns) and adults exhibited a larger SRE than children, whose selfconcept is presumably less well-developed (e.g., Halpin, Puff, Mason, \& Marston, 1984). 
A main goal of the present research was therefore to examine whether individual differences in personality self-concept relate to measures of the SRE, in particular by appealing to the causal underlying processes of elaboration and organization in memory.

\section{Measures of Elaborative and Organizational Processing}

As was briefly discussed earlier, measures of elaborative processing have been heavily criticized (e.g., Baddeley, 1978; Eysenck, 1978) for being circular, in that both the cause and effect are evidenced by the same measure (i.e., amount recalled). Specifically, the self-reference effect is evidenced by a higher amount recalled and measures of elaborative processing are also evidenced by amount recalled. As a result, concerted efforts have been put forward to devise measures of elaboration and organization that are independent from amount recalled.

Within the literature on the SRE, Klein et al. (1994) demonstrated alternative measures of elaborative and organizational processing that require participants to undergo two subsequent free recall tasks (see also Burns, 1993; Klein, Loftus, Kihlstrom, \& Aseron, 1989). With two recall tasks, they measured the percentage of items recalled on the second task that were not recalled on the first task (item gain) and the percentage of items recalled on the first task that were not recalled on the second task (item loss). Item gain was argued to be due to elaborative processing, in which the set of idiosyncratic retrieval cues (i.e., item-specific information) acquired during encoding are not exhausted on the first recall test, and therefore when given a second test, those cues have an opportunity to elicit successful retrieval of new list items. In contrast, a lack of item loss was argued to be due to organizational processing, wherein the small set of retrieval cues that encoded the similarity of the items (e.g., category labels or shared attributes) used in 
the first recall task are still available in memory when facing the second recall task (e.g., a retrieval scheme), thus protecting against item loss. Klein et al. (1994; Experiment 1) found a SRE using the repeated-testing paradigm. The new measures suggested that organizational processing was responsible for the effect, but there was also a trend for an increase in item gain, which suggested that elaborative processing may have also contributed to the effect ( $n=20$ in their study provided limited power to detect even medium-sized effects).

Although the repeating-testing approach was indeed an improvement over the amount recalled measure of elaborative processing, Burns and Hebert (2005; Burns \& Schoff, 1998) levelled a serious criticism against it. Specifically, item gain clearly depends on the amount of time researchers allow for recall in each memory test. A more precise measure of when recall happens during a memory test (i.e., the time course of recall) is provided by cumulative recall curves (Burns \& Schoff, 1998; Wixted \& Rohrer, 1994). In a plot of the amount of unique items recalled as a function of time (e.g., in minutes), cumulative recall curves are best described by the rate of approach to asymptote and the level of asymptotic recall. Specifically, the curve is best described by the following exponential function,

$$
n(t)=n(\propto)\left(1-e^{-\lambda t}\right)
$$

where $n(t)$ is number of items recalled at time t, $n(\propto)$ is the asymptotic level of recall, and $\lambda$ is the rate of approach to asymptotic recall. The rate of approach provides insight into the amount of elaborative processing engaged during encoding. More precisely, a shallow curve (a low rate of approach) generally suggests that items were recalled more slowly throughout the recall period. In contrast, a steep curve (a high rate of approach) 
generally indicates that most recalled words were produced relatively early in the recall period, which suggests the use of effective relational information acquired by organizational processing (i.e., by using relevant categories to narrow the search of memory). The fact that research typically shows that the rate of approach to asymptote is negatively related to asymptotic recall (see Wixted \& Rohrer, 1994), with shallow recall curves typically showing higher asymptotic recall, rightfully puts into question whether the measure is actually independent from amount recalled. However, Burns and Schoff (1998; see also Burns \& Hebert, 2005; Wixted \& Rohrer, 1994) have found many qualitatively different exceptions to the common finding that the rate of approach is negatively related to asymptotic recall, all of which were motivated by hypotheses derived from the item-specific relational memory framework (Hunt \& McDaniel, 1993). A review of their work comparing cumulative recall curves for conditions in which itemspecific and relational processing were manipulated helps to form an understanding of the impact of those processes on the time course of recall (see Figure 1). 

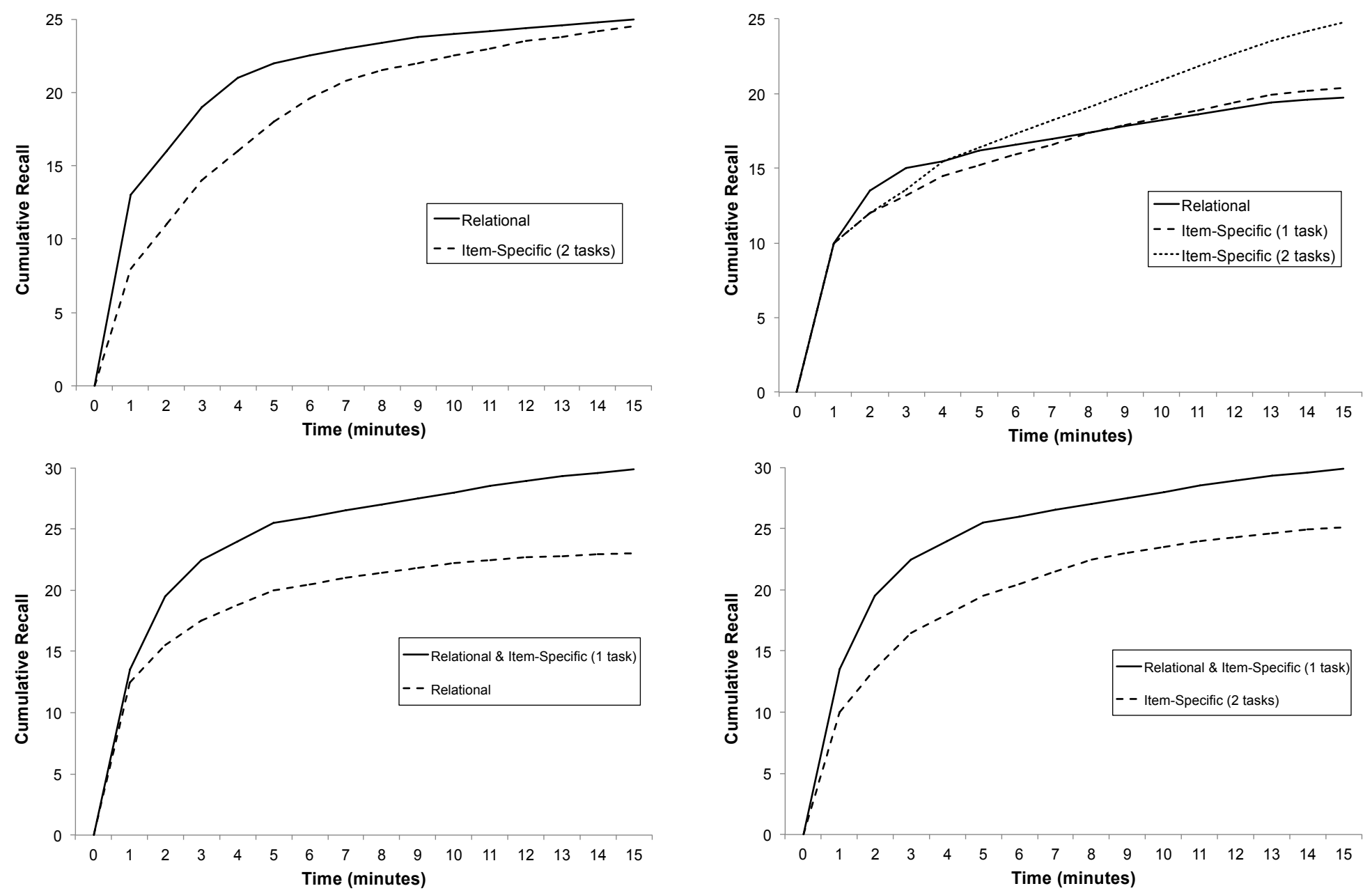

Figure 1. Cumulative recall as a function of various combinations of item-specific and relational processing (adapted from Burns \& Schoff, 1998). Top left $=$ Experiment 1; Top right $=$ Experiment 3 ; Bottom left $=$ Experiment 4; Bottom right $=$ Experiment 5 . 
In Burns and Schoff's (1998) first experiment, participants encoded a list of items belonging to one of seven ad hoc categories (i.e., an unrelated list) through relational processing (category sorting task) or item-specific processing (pleasantness rating and then bringing to mind an associated word) and were given one free recall task lasting 15 minutes (see top left panel in Figure 1 for the pattern of cumulative recall results). The rate of approach to asymptote was significantly higher in the relational processing condition (i.e., faster recall), but asymptotic recall did not differ between conditions (hence, recall speed was not negatively associated with amount recalled). Burns and Schoff's second experiment replicated this pattern with the item-specific condition engaging in pleasantness rating and then bringing to mind two associated words instead of one. Interestingly, the results of both experiments showed that if a long recall period is provided, the recall advantage of relational processing over item-specific processing on an unrelated list of items (as was observed in Einstein \& Hunt, 1980) vanishes, because the item-specific group is allowed enough time for successful retrieval of list items later in the recall period (i.e., if recall was stopped at minute 5, there would be a clear recall advantage for the relational processing condition; however, if it was stopped at minute 15 , the recall advantage would disappear).

Burns and Schoff's (1998) third experiment compared a relational processing condition to two item-specific conditions, with participants in the first item-specific condition instructed to form an image of each word and those in the second condition instructed to form an image of each word and then bring to mind an associate. The top right panel of Figure 1 presents the qualitative pattern observed in the cumulative recall results. Analysis of rate of approach showed that recall was fastest following relational 
processing, slower following the item-specific condition with one encoding task, and slowest following the item-specific condition with two encoding tasks. Furthermore, the item-specific condition with two tasks had a significantly higher level of asymptotic recall than both the relational and item-specific condition with only one encoding task, and the latter two conditions did not differ, showing yet again that recall speed can be slow without rendering higher recall. Thus far, one can see in the plots of cumulative recall that relational processing produces faster recall, in that item gains occur relatively early in the recall period, whereas item-specific processing produces slower recall, with item gains occurring relatively late in the recall period.

Burns and Schoff's (1998) fourth experiment compared a relational processing condition (category sorting) to a condition that combined relational and item-specific processing (category sorting and pleasantness rating). The qualitative pattern of the results is presented in the bottom left plot of Figure 1, where a clear recall advantage can be seen in the combined relational and item-specific condition. The rate of approach to asymptote, however, did not differ between the conditions (i.e., another exception to the commonly observed negative relationship between recall speed and amount recalled). An additional analysis showed that significantly more items were gained from minute 5 to 15 in the combined condition than in the relational processing only condition. These results suggest that item-specific processing in the context of relational information leads to continuously effective retrieval of list items throughout the recall period, most notably early-on (e.g., minutes 2 and 3) as well as in the later portion (e.g., minutes 10 through 15). The fact that the two conditions differed in amount of item-specific processing but not in the rate of approach to asymptote highlights the importance of looking more 
closely at the pattern of cumulative recall to interpret differences in item-specific processing. In this case, the additional recall of items both early and late in the recall period may have obscured the typical slowing of recall associated with increased levels of item-specific processing (but the item gain measure from minute 5 to 15 was able to capture this difference).

Burns and Schoff's (1998) fifth experiment compared an item-specific processing condition (pleasantness rating) to a condition that combined relational and item-specific processing (category sorting and pleasantness rating). The qualitative pattern of the results is presented in the bottom right plot of Figure 1. The rate of approach to asymptote and level of asymptotic recall were both higher in the relational and itemspecific condition than in the item-specific only condition (i.e., recall speed and amount recalled was positively related). Furthermore, a similar number of items were gained in the two conditions between minutes 5 and 15. These results suggest that relational information in the context of item-specific information serves to speed up successful retrieval of list items only in the early portion of the recall period (e.g., minutes 1 through 3). Hence, the fifth experiment showed that the addition of relational information when there was item-specific processing served to increase recall speed (as one would expect), whereas in the fourth experiment the addition of item-specific processing when there was relational information did not significantly impact recall speed (contrary to what would be expected). This can be understood as relational information generally impacting item retrieval early in the time course of recall and item-specific information generally impacting item retrieval early and late in the time course of recall. 
The results of Burns and Schoff's (1998) experiments are clear exceptions to the common finding that higher levels of recall are negatively associated with recall speed, suggesting that recall speed can be a measure of elaborative processing that is independent from amount recalled. It is also clear that a measure of item gain may or may not detect a difference between encoding conditions depending on when during the time course of recall the measure is taken (e.g., for the top left plot of Figure 1, a measure of item gain from minute 5 to 15 would show that the item-specific condition rendered much higher item gain than the relational condition, however if item gain was compared later in the recall trial, say at minute 13 to 15 , the difference would be much smaller and perhaps not be significant). Their research highlights that cumulative recall is affected by a complex interplay of item-specific and relational processing, and that researchers must examine not only the estimates of the best fitting cumulative recall curves but also plots of cumulative recall to understand the interplay. A final note is that Burns and colleagues have been successful in applying cumulative recall curve analysis to various areas of memory research (false memory: Burns, Jenkins, \& Dean, 2007; Burns et al., 2006; proactive interference: Burns, 1989; free recall: Burns \& Hebert, 2005; Burns \& Schoff, 1998; and the survival processing effect: Burns, Hwang, \& Burns, 2011), supporting the utility of the approach more generally.

In sum, although Klein et al. (1994) introduced the item gain and item loss measures to better understand the nature of the SRE, and indeed these circumvented the circularity of previous measures of elaborative processing (i.e., amount recalled), Burns' (1993; Burns \& Hebert, 2005; Burns \& Schoff, 1998) criticism of the item gain measure and his empirical findings cannot be ignored. Within the literature on the SRE, 
researchers have not yet conducted a detailed examination of cumulative recall as a way to better understand the contributions of elaborative and organizational processing to free recall, and how those measures vary as a function of orienting tasks such as semantic, self-reference, and other-reference. Given that the evidence strongly suggests selfreference encoding elicits item-specific and relational processing, Burns and Schoff's (1998) patterns of cumulative recall will be informative for interpreting personality differences in recall speed and asymptotic recall of corresponding self-referenced adjectives. To my knowledge, the present research comprises the first set of studies to take this new approach in an effort to explore the nature of the SRE.

\section{Does Self-Reference Recall Performance Tell Us Something About Personality?}

The initial demonstration of the SRE inspired researchers due to its potential for a new cognitive paradigm that could be adopted to study the self-concept (Rogers et al., 1977; or self-schemata, Markus, 1977). Studies in numerous disciplines in psychology continued to explore that possibility ranging from clinical applications (e.g., Bradely, Mogg, Galbraith, \& Perrett, 1993; Young \& Martin, 1981) to neuroscientists attempting to locate the self in the brain (e.g., Craik et al., 1999; Kelly et al., 2002; Kircher et al., 2000; Klein, 2012a provides a recent review). Klein and colleagues' influential work reviewed earlier on the processing involved in the SRE, however, not only cast doubt on whether the SRE is relevant to self-concept (e.g., it could be exclusively explained by organizational processing, Klein \& Kihlstrom, 1986), but changed the focus from selfconcept to memory processes (Symons \& Johnson, 1997). I argue that these foci should be considered together, as information processing must act on some memory structure. In 
a retrospective review of the levels-of-processing framework and the research it inspired, Lockheart and Craik (1990) stated:

The self-reference phenomenon comprises precisely the kind of data needed to build a comprehensive theory of memory encoding; and it was the levels framework that stressed that its explanation was to be found in an analysis of the particular meaning-extracting operations involved in performing the orienting task. Obviously such an analysis, if it is to isolate those principles that are important for remembering, may require considerable research into the structure of the self-concept. (p. 93)

Einstein and Hunt (1980) also highlighted the importance of considering the structure of memory when introducing their framework that combined levels-of-processing and organization in memory, and they stated:

The guiding framework for this research involves consideration of encoding process and structure at encoding (Hunt, Elliot, \& Spence, 1979). The structural representations of relational and individual-item information are available in semantic memory and the information activated as the representation of a particular event is determined by the encoding process. Both processes and structure are necessary considerations. (p. 597)

The present research puts forward a concerted effort to bring together the causal memory processes involved in the SRE and the structure of self-concept. In doing so, it employed a novel analysis of processing by examining cumulative recall curves to assess individual differences in cognitive elaboration and the item-specific information it activates in memory. To connect those individual differences with research on the self-concept, I turn 
to the personality and social cognitive literatures to provide some insight on the particular dimensions of personality represented in the self-concept (i.e., structure), and how those dimensions may be measured.

\section{Direct Personality Measures}

Personality researchers have long assumed that the language people use to describe themselves and other people provide insight into dispositional personality traits (e.g., Allport \& Odbert, 1936, Norman, 1967, Goldberg, 1993). Following this lexical approach to discovering traits, people indicate to what extent a large corpus of personality adjectives (e.g., organized, precise, and orderly) describe who they are (self-report) or who someone else is (other-report) using a rating scale (e.g., from 1 [Strongly Disagree] to 5 [Strongly Agree]). Researchers employ factor analytic techniques to determine whether a small number of latent factors adequately account for the pattern of intercorrelations among the ratings. For instance, a series of items within a personality questionnaire that purport to measure one latent personality trait (e.g., conscientiousness) should receive consistent values on the response scale within individuals. For example, someone who is highly conscientious should respond consistently at the high end of the response scale across items describing conscientiousness whereas someone with an average standing on the trait should respond consistently in the middle. In essence, proponents of the lexical approach strive to determine whether a small number of latent personality traits exist by examining ratings of self- or other-descriptiveness for a series of personality adjectives, decisions that inevitably come from ones conception of one's self or of someone else. 
Over at least 50 years of rigorous research (e.g., since Tupes and Christal, 1961), personality psychologists have repeatedly found support for a five-factor model, suggesting there are five broad dispositional traits (i.e., the Big Five; Goldberg, 1993 provides an excellent historical review). Nevertheless, some critics have argued and found support for more or fewer broad factors. For example, De Raad and Peabody (2005) found that only three traits appear consistently across a variety of cultures, suggesting the three-factor model is more generally applicable. Contrariwise, Lee and Ashton (2004) have found support for a six-factor model. Perhaps the biggest critic is Block (1995, 2010), who has argued forcefully that the general approach is limited. Notwithstanding the critics, the Big Five has surfaced in numerous studies implementing self-reports and other-reports obtained with different samples within different cultures therefore supporting its significance. It also surfaces when people rate dispositional phrases (e.g., "like order and regularity") for self- or other-descriptiveness in a standard questionnaire format, which was popularized by McCrea and Costa (1987). Although there is some variation in how the five factors have been labeled, McCrea and Costa's labels capture the general essence: extraversion, neuroticism, conscientiousness, agreeableness, and openness to experience, with the latter label otherwise called intellect or culture. Researchers generally agree that these broad traits are superordinate to a constellation of narrower facets (e.g., conscientiousness can be more specifically characterized by self-efficacy, orderliness, dutifulness, achievement-striving, selfdiscipline, and cautiousness; Goldberg et al., 2006). Across the various studies that support a different number of factors, there is some consistency in the interpretation of observed factor structures. Conscientiousness, for example, surfaces in the three-, five-, 
and six-factor models. The consistency across studies is highly suggestive of stable psychological personality trait dimensions.

Aside from the statistical component of personality trait assessment (i.e., factor structure), construct validity must be established through convergent, divergent, and predictive validity. That is, similar traits should be related, dissimilar traits should be unrelated, and perhaps more importantly, traits should predict behaviour that is deemed relevant by theory or through an a priori analysis (e.g., a sample of individuals agree the behaviour is relevant to the trait). Indeed, a great deal of personality research has been conducted in an effort to predict behaviour, whether self-reported (e.g., "do you enjoy reading classic literature?") or observed (e.g., lateness to an appointment). For example, Paunonen and Ashton (2001a) asked whether 717 students' final grade in a personality psychology course relates to conscientiousness and openness to experience, and the respective facets. They found that conscientiousness was significantly related to obtained final grade $(r=.21)$, as was the facet of achievement $(r=.26$; the difference between these correlations was not significant). On the other hand, openness to experience was not related to obtained final grade $(r=-.04)$, but the facet of understanding was significantly related to final grade ( $r=.23$; the difference between these correlations was significant). This latter result in particular bolstered the authors' argument that facets may be better predictors of behaviour than broad traits under certain circumstances (see also Ashton, 1998, Paunonen, 1998, 2003; Paunonen \& Ashton, 2001b, Aviv, Zelenski, Rallo, \& Larsen, 2002). Nevertheless, the predictive validity of broad traits such as conscientiousness, and narrower facets, may be established through their prediction of relevant behaviour. 
Another line of research relating behaviour and personality examines participation in a university subject pool. Aviv, Zelenski, Rallo, and Larsen (2002) found that conscientiousness, and a subset of its facets (order, dutifulness, and self-discipline), were related to the week during the semester when individuals participated in lab experiments. Specifically, conscientious individuals participated earlier in the semester relative to those who were less conscientious ( $r=-.14$; and the facets: order, $r=-.18$; dutifulness, $r$ $=-.15$; self-discipline, $r=-.13$; see also Witt, Donnellan, \& Orlando, 2011; and more recently, Ebersole et al., 2015). In a similar vein, Zelenski, Rusting, and Larsen (2003) found that conscientiousness was related to the time of day when individuals participated in an experiment, with more conscientious individuals participating earlier in the day than those who are less conscientious $(r=-.23)$. Finally, Back, Schmukle, and Egloff (2006) examined the relationship between personality and punctuality when attending an experiment (i.e., time of arrival, earliness, and lateness). They found that conscientiousness predicted all three punctuality measures above and beyond gender and age, and independent from the other Big Five traits.

On the whole, observed behaviour is a more persuasive criterion for predictive validity of personality traits than is self-reported behaviour. When the same individual self-reports both the personality and behaviour measures, an observed relationship may be due to demand characteristics of the study. For instance, when participants realize or are told the study concerns the relationship between conscientiousness and academic performance, they may distort their personality and behaviour responses so the two coincide. Furthermore, the nature and wording of the questions from the two measures may promote consistent responding (e.g., when a participant is 20 minutes late and the 
personality question asks “are you punctual?"). Another concern more generally is socially desirable responding (e.g., responding in a way that conforms with social norms or appears favourable; Crowne \& Marlowe, 1960; Paulhus, 1984). However, if the behavioural measure is observed (or inter-subjectively judged) or an observer assesses personality (e.g., other-reports), these concerns may be reduced. Of course, if one collects observed behaviour and other personality reports, the concern may be eliminated entirely depending on the specific details of the procedure (e.g., raters who are blind to the study's purpose). An interesting alternative to observer personality reports, however, has been developed in the social cognitive literature.

\section{Indirect Personality Measures}

Personality self-reports in the social cognition literature have been dubbed a direct measure of personality (e.g., De Houwer, 2006), thought to provide access to an explicit personality self-concept (Schnabel \& Asendorpf, 2010). Researchers have been concerned about two potential sources of bias when personality is measured directly. Firstly, the human ability to introspect (consciously bring to mind past experiences or beliefs) does not allow access to all potentially relevant sources of information when making self-descriptiveness judgments. Secondly, as mentioned above, there is evidence to suggest that measurements may be distorted by demand characteristics and selfpresentation biases (e.g., impression management and socially desirable responding). In an effort to circumvent these issues, social cognitive researchers have developed indirect personality measures, which are thought to provide access to an implicit personality selfconcept. The most widely studied indirect personality measure is the IAT (Greenwald, McGhee, \& Schwartz, 1998). 
In the case of an IAT designed to measure conscientiousness (conscientiousnessIAT, e.g., Grumm \& Collani, 2007), participants undergo a series of categorization trials organized into blocks. In the first block, participants classify stimuli (e.g., me, self, I, others, them, their) into one of two categories (concepts), labeled "Me" and "Not Me" for example. In the next block, participants classify trait adjectives (e.g., organized, efficient, punctual, sloppy, disorderly, careless) into one of two contrast categories (attributes). Typically, the two poles of one personality trait dimension serve as the attributes (e.g., "Conscientious" and "Not Conscientious"). In a series of subsequent blocks, participants categorize instances of the concepts and attributes using only two response keys, and the response key assignments change across blocks (e.g., press the left response key when you see stimuli from the "Me" or "Conscientious" categories, and the right response key when you see stimuli from the "Not Me" or "Not Conscientious" categories). In another block, the response key assignment for the attributes is reversed (e.g., press the left response key for "Me" and "Not Conscientious" stimuli and the right response key for "Not Me" and "Conscientious" stimuli). The categorization response time difference for the attribute stimuli presented in the two blocks described above provides what is known as the IAT effect (Greenwald, Nosek, \& Banaji, 2003, present various scoring algorithms). When the IAT effect is large, participants' categorization is faster when they classify attribute stimuli ("Conscientious" adjectives) with the same response key as one concept ("Me") relative to the other ("Not Me"). In this way, the IAT purports to measure the relative strength of associations in memory between the concepts ("Me" vs. "Not Me") and the attributes ("Conscientious" vs. "Not Conscientious"). In this case, when the effect is large, the individual has high implicit conscientiousness. 
Recent efforts have been made to examine the validity of Big Five IATs for measurement of implicit personality self-concept (Grumm \& Collani, 2007; Schmukle, Back, \& Egloff, 2008). The results generally show that they have satisfactory internal consistency, poor to moderate convergent validity (i.e., low to medium correlations with self-reports), and fair divergent validity (i.e., the IATs tend to be uncorrelated), and moderate test-retest reliability. The poor to moderate convergent validity in particular suggests that indirect measures may tap a different aspect of personality self-concept than do direct measures. In support of that notion, researchers have found that Big Five IATs have incremental validity over self-reports in predicting trait related behaviour and in predicting relevant behaviours that are difficult to control. Specifically, Egloff and Schmukle (2002) validated an anxiety IAT and found it had incremental validity over a self-report measure in predicting anxiety related behaviours elicited by perceived task failure and public speaking. Asendorpf, Banse, and Mucke (2002) found that a shyness IAT, but not a self-report, predicted shyness behaviours that are difficult to control while self-reported shyness, but not the IAT, predicted shyness behaviours that are more easily controlled (i.e., a double dissociation). Steffens and Schulze-König (2006; see also Steffens, 2004) administered a test of attention and concentration (d2 test; Bates \& Lemay, 2003) in which participants crossed out the letter "d" when it appeared with two marks among a sheet filled with "d"s and "p"s with 1 to 3 marks. They found that a conscientiousness-IAT predicted fewer mistakes on the $\mathrm{d} 2$ test, but self-reported conscientiousness was unrelated to performance. Furthermore, Schmukle, Back, and Egloff (2008; see also Back, Egloff, \& Schmukle, 2009) reported incremental validity of Big Five IATs for predicting various self-reported behaviours. For instance, frequency of 
window cleaning was related to a conscientiousness-IAT $(r=.24)$ after controlling for self-reported conscientiousness and gender. Beyond establishing the predictive validity of Big Five IATs as implicit measures of personality self-concept, these studies suggest that personality IATs are able to predict behaviours that are not captured by a direct personality measure, supporting their construct validity.

From a theoretical standpoint, it is important to understand what direct and indirect personality tests measure. Dual-process models such as Gawronski and Bodenhausen's (2006, 2011, see also Strack \& Deutsch, 2004) association-propositional evaluation (APE) model offers an account (Schnabel \& Asendorpf, 2010, provide an overview of how the account is relevant to self-concept research). They suggest that indirect tests such as IATs measure the relative activation of associations in memory when people are presented a stimulus. In contrast, direct tests measure the outcome of propositional reasoning, where a subset of the propositions considered are brought to mind by activated associations (e.g., upon seeing the questionnaire item "like order", a "Me-Disorderly" association may be activated giving rise to the proposition "I am disorderly"). Other propositions, however, may come to mind and factor into the propositional reasoning process, yielding a response that may or may not be congruent with activated associations. For instance, in a job interview, the propositions "I need this job" and "they want an employee who is organized" may come to mind, which could lead to negation of the proposition "I am disorderly" when presented the item "like order" on a questionnaire. The negation of such a proposition in favour of the other two would ultimately lead to a high value on the self-report measure of conscientiousness (i.e., a self-presentation bias). An indirect measure, however, would capture the "Me- 
Disorderly" association that gave rise to the negated proposition ("I am disorderly") and yield low implicit conscientiousness. Hence, direct and indirect measures of conscientiousness in this case would be dissociated. In this way, the APE framework provides an explanation for the low to moderate convergent validity between direct and indirect personality measures (Grumm \& Collani, 2007; Schmukle, Back, \& Egloff, 2008).

Aside from inconsistent correlations between implicit and explicit measures, the APE model (Gawronski \& Bodenhausen, 2006, 2011) also provides an explanation for the incremental validity and double dissociations observed between implicit and explicit personality self-concept in predicting behaviour. In particular, the model suggests Big Five IATs measure the relative strength of associations between the self-concept and the Big Five dimensions while self-report measures are based on the outcome of propositional reasoning, which may or may not be congruent with the propositions implied by the activation of associations in memory. When individuals are placed in a context where they must act spontaneously (or in a less controlled manner), it may be unlikely that they could engage in propositional reasoning to guide their behaviour. Hence, implicit measures would predict those behaviours and explicit measures that are taken under conditions where individuals are given all the time required to select their responses would be less likely to predict those behaviours. In support of that notion, Hofmann et al.'s (2005) meta-analysis found that the correlation between implicit and explicit measures increases with the spontaneity with which explicit measures are taken. In contrast, when individuals are put in a context where they can deliberate before behaving, the explicit measures would better predict those behaviours than the implicit 
measures (e.g., Asendorpf, Banse, \& Mucke, 2002). Hence, in this way, the APE model provides an explanation for a fair amount of data on explicit and implicit personality selfconcept and the behaviours predicted by those measures.

A review of the literature on personality self-concept suggests that the present research would profit from collecting both direct and indirect measures. It was not clear a priori whether measures of elaborative processing in a self-reference task would be related to a corresponding direct or indirect measure. Nevertheless, when participants make a self-descriptiveness judgment in a self-reference task, presumably the same type of processing and structure would be involved in rating adjectives on a direct personality measure (e.g., Goldberg, 1993). If that is the case, I expected the number of endorsed trait adjectives (e.g., conscientiousness adjectives) in the self-reference task to be highly correlated with a validated direct measure. However, it was not clear whether the measure of elaborative processing (i.e., the steepness of recall curves) would be related to the outcome of the propositional reasoning that lead to the self-descriptiveness judgment (i.e., a direct measures) or to the activation of associations in memory elicited by the stimuli (i.e., an indirect measure). If the IAT is in fact a proxy measure of how strongly the self-concept is related to a personality trait, I expected that people who demonstrate a strong implicit trait would also have shallow recall curves for corresponding trait adjectives encoded self-referentially. However, if the propositions elicited by the activation of associations are more important cues for recall of self-referenced adjectives, I expected a direct measure would predict the steepness of those recall curves. It was also possible that both direct and indirect measures would independently relate to the curves. On an exploratory basis, and in an effort to bring together the literature on personality 
self-concept and assessment, the present research included both types of personality measures. Moreover, behavioural indicators were included to assess the predictive validity of the memory-based personality measures.

\section{The Present Research}

To test the ideas put forward, I conducted three studies that examined the main tenet of the thesis including replications and extensions. In all three studies, there was a levels-of-processing task with repeated-testing of free recall, a recognition test, direct and indirect trait measures, and measures of trait related attitudes and behaviours. The levelsof-processing task in the first study closely followed Rogers et al.'s (1977) procedure. Participants were presented one of four different orienting questions designed to induce shallow to deep levels of processing of trait adjectives (structural, rhyming, semantic, and self-reference). All adjectives described two facets of conscientiousness, namely orderliness and impulse control (De Raad \& Peabody, 2005). The adjectives that described the positive pole of orderliness were assigned to the self-reference task, those describing the positive pole of impulse control were assigned to the semantic task, and the adjectives describing the negative pole of orderliness and impulse control were assigned to the rhyming and structural encoding tasks respectively.

The adjectives in all studies were carefully selected using a multi-faceted approach. Specifically, adjectives were selected from lexical research on the factor structure of traits (De Raad \& Peabody, 2005; Goldberg, 1990; Johnson \& Ostendorf, 1993; Trapnell \& Wiggins, 1990) and research on Big Five IATs (Schmukle, Back, \& Egloff, 2008; Steffens, 2004; Steffens \& Schulze-König, 2006). In addition, the latent semantic association between the adjectives and the facets was taken into account 
(Landauer, Foltz, \& Laham, 1998). To eliminate confounding variables on recall and recognition among the words presented within each encoding task, the four lists of adjectives assigned to the encoding tasks were equated for word frequency, word length, and meaningfulness. In addition, the adjectives between the positive poles and the adjectives between the negative poles of the facets were equated for likeableness. Hence, any observed differences in recall and recognition between the self-reference and semantic orienting tasks was not due to such extraneous variables.

Following the levels-of-processing task, participants completed a 3-minute selfpaced $\mathrm{d} 2$ test of concentration and attention (Brickencamp \& Zillmer, 1998). Steffens and Schuzle-König (2006; Steffens, 2004) found that a direct measure of conscientiousness was unrelated to the number of cancellation errors made in a self-paced $\mathrm{d} 2$ test, but reported a negative relationship between number of errors committed and a conscientiousness-IAT. Hence, aside from acting as a brief filler activity typical of studies examining free recall, it served as a behavioural indicator of implicit conscientiousness. Immediately following the d2, two consecutive 5-minute surprise memory tests were administered (e.g., Burns, 1993; Klein et al. 1994). Every minute, participants made a mark beside the last word they recalled, which allowed for calculation of recall over time by fitting cumulative recall curves (e.g., Burns \& Hebert, 2005; Burns \& Schoff, 1998). This procedure also allowed for an assessment of item gains, item losses, and the cumulative recall curve parameters presented in Equation 1.

Following free recall, participants engaged in a complex reading span task in which they judged whether a series of sentences made sense or not. After making their judgment, a capitalized letter was briefly presented. After a certain number of judgments, 
participants were cued to recall the capitalized letters in the presented order. This task has been shown to capture an important aspect of working memory capacity that describes participants' general ability to store information in memory while completing a secondary processing task (Conway et al., 2005). Individual differences in this measure of working memory capacity have been shown to influence the parameters of cumulative recall curves (Unsworth \& Engle, 2007). In particular, people who have a high working memory capacity display a higher approach to asymptote and higher asymptotic recall than those with low working memory capacity. The authors suggested that participants with a low working memory capacity are not able to constrain their memory search for recall cues as well as those with a high working memory capacity. Hence, the complex reading span measure served as a control variable for analyses involving the rate of approach to asymptote and a filler task between the recall and recognition tests.

The recognition memory test was composed of the trait adjectives presented in the level-of-processing task (old items), semantically related adjectives that were not presented (lures), and adjectives that described physical features (unrelated items; e.g., descriptors along the dimensions of height, weight, and strength). The recognition data allowed for a secondary assessment of the SRE, and more importantly, a secondary assessment of the item-specific and relational information encoded during the levels-ofprocessing task. In particular, encoding item-specific information has been shown to prevent false recognition of lures (Einstein \& Hunt, 1980; Hunt \& Einstein, 1981; McCabe et al., 2004).

For an indirect personality measure, an IAT was completed. In Study 1, there were two IATs. The first IAT was constructed using the orderliness adjectives presented 
in the level-of-processing task (orderliness-IAT). The second was constructed using the impulse control adjectives (impulse control-IAT). For a direct personality measure, a questionnaire comprised of validated scales measuring conscientiousness, orderliness, impulse control, extraversion, neuroticism, and openness to experience was completed, along with another questionnaire tapping self-reported attitudes and behaviours associated with these measures (e.g., Puanonen, 1998, 2003). In addition, the time of day, week within the semester, and lateness were recorded as measures of conscientious behaviour (Aviv, Zelenski, Rallo, \& Larsen, 2002; Back, Egloff, \& Schmukle, 2009; Zelenski \& Larsen, 2003).

Study 2 was largely a replication of the Study 1, with a few important changes. It was designed to determine whether any observed relationship among measures of elaborative processing and personality in Study 1 was due to orderliness or conscientiousness behaviour per se (as opposed to differences in the representations comprising personality self-concept). Given the nature of these traits, it was possible that highly orderly people put forward a more consistently high level of effort in the memory tasks than those who were less orderly. Put another way, orderly individuals may have approached the task differently than disorderly individuals. To rule out that possibility, the trait adjectives encoded in the self-reference condition described imaginativeness, a facet of openness to experience (Goldberg et al., 2006), which allowed for comparisons of direct and indirect measures of orderliness to the rate of approach to asymptote following self-reference encoding of imaginativeness adjectives. If orderly people indeed adopted the aforementioned strategy, I expected that measures of orderliness would relate to the rate of approach parameter for the self-reference task and to false recognition. In 
contrast, if such a relationship does not exist, the results would cast doubt on whether the observed relationship in Study 1 was due to an orderly approach to recall and recognition per se. Moreover, these methodological changes allowed for a generalization of the observed relationships with orderliness adjectives in Study 1 to imaginativeness adjectives in Study 2 (i.e., imaginativeness was expected to relate to the rate of approach parameter for the self-referenced imaginativeness adjectives).

Another potential concern regarding Study 1 was that the direct measures of personality were administered at the end of the study, when participants may have determined that the study was about personality measurement. This realization may have rendered a demand characteristic, which may have distorted direct measures of personality and self-reported behaviour. Therefore, in Study 2, the direct personality measures were collected in the mass-testing questionnaire administered early in the Fall 2012 semester, instead of in the lab. Any observed association between the direct measures and the critical variables of interest (i.e., recall curves, item loss, false recognition, IATs, attitudes, and behaviour) in Study 2 were therefore likely not due to demand characteristics of the materials and procedure. Moreover, this change determined whether the relationships of interest could be obtained when the measures were separated in time and space.

Study 3 was a close replication of the first two studies, but offered an important extension. A new encoding task that has been shown to produce especially high free recall and recognition replaced the two shallow tasks (i.e., the structural and rhyming encoding tasks). Specifically, the other-reference task was included, which required participants to encode the trait adjectives in relation to a close other (i.e., "Describes my 
best friend?"). In Symons and Johnson's (1997) meta-analytic review, they found that the SRE is diminished, but still significant, when comparing recall and recognition from selfreference to an other-reference task. The authors suggested that the other-reference task yields both elaborative and organizational processing, particularly when the task requires tapping into knowledge of an intimate other. This encoding task was particularly well suited for Study 3 because direct personality measures for the other-referent individual (i.e., the best friend) were fairly easily obtained from the participant. Perhaps most importantly, the other-reference task tested the generality of the relationship between elaborative processing and personality by tapping into a memory structure other than the self-concept. Study 3 therefore had the potential to provide converging evidence that the encoding task (self-reference or other-reference) taps a knowledge structure that can be evaluated by the extent to which item-specific information is evidenced in free recall and recognition. Hence, it allowed for an assessment of whether the self-concept (or selfschemas; Markus, 1977) necessarily has special properties, as has been suggested in past research on the SRE (Katz, 1987; see Klein, 2012a for a recent discussion and review; see Bower \& Gilligan, 1979 for an alternate view), or if it is just a more complex memory structure (i.e., with more item-specific and relational information).

\section{Hypotheses}

\section{Self- and Other Reference Effects}

I expected a main effect of encoding task on proportion recalled and correct recognition in all three studies. The main effect was expected to show that self-reference encoding produces a higher proportion recalled and correct recognition of adjectives presented in the levels-of-processing task than all other encoding tasks (including the 
other-reference task in Study 3; Symons \& Johnson, 1997). The other-reference task was expected to yield a higher proportion recalled and correct recognition than the semantic task, whereas the semantic task was expected to produce a higher proportion recalled and correct recognition relative to the structural and phonetic tasks in Study 1 and 2 (i.e., a levels-of-processing effect).

Predictions concerning item gain and item loss assumed that the levels-ofprocessing and self-reference effects were due to higher involvement of elaborative and organizational processing in deeper encoding conditions (Symons \& Johnson, 1997; Klein \& Loftus, 1989; Klein et al., 1994). Specifically, I expected a main effect of encoding task on item gain, wherein self-reference would produce higher item gain than semantic encoding, which in turn would produce higher item gain than structural and phonetic encoding. The other-reference task in Study 3 was expected to yield lower item gain than the self-reference task but higher item gain than the semantic task. The main effect of encoding task on item loss was expected the follow the same pattern as the item gain results, except in the opposite direction (i.e., item loss would be highest for the structural and phonetic tasks, relatively lower in the semantic task, even lower in the other-reference task, and finally, lowest in the self-reference task).

\section{Personality and Self- and Other-Reference}

The core idea put forward required an examination of the inter-correlations among measures of elaborative and organizational processing and direct and indirect personality measures. Zero-order and partial (controlling for working memory capacity) correlations among these measures were examined. Across the three studies, I hypothesized significant negative partial correlations of measures of elaborative 
processing of self-referenced trait adjectives (rate of approach to asymptote and false recognition) with corresponding direct or indirect personality trait measures. The pattern of inter-correlations was expected to only surface following self- or other-reference encoding and when the encoded trait adjectives matched a directly or indirectly measured trait (of the self or a best friend, respectively). That is, self- and other-reference judgments were hypothesized to be necessary for the patterns to emerge and the patterns were only expected to emerge when the encoded trait adjectives matched the personality measures (i.e., self- or other-reports of matching traits).

More specifically, in Study 1, I expected negative partial correlations between the direct or indirect personality measures and the rate of approach to asymptote of selfreferenced adjectives and false recognition. People who self-reported as being highly orderly and conscientious or showed strong orderliness-IAT effects were expected to recall the self-referenced orderliness adjectives more slowly across the two recall tasks and falsely recognize fewer conscientiousness adjectives than people who were lower on the direct or indirect measures (i.e., because more item-specific information concerning the trait was expected to be available within the self-concept). Furthermore, these patterns were expected to emerge after statistically controlling for participants' working memory capacity. In Study 2, when the imaginativeness adjectives were self-referenced instead of the orderliness ones, I expected the same pattern of partial correlations to emerge, but only with the direct or indirect measures of openness and imaginativeness, not conscientiousness or orderliness. That is, the pattern of significant partial correlations from Study 1 among the measures of orderliness and recall and recognition following self-reference were not expected to be significant, whereas those relationships were 
expected to be significant when direct and indirect measures of imaginativeness were examined, supporting the convergent and divergent validity of the memory-based measures (i.e., an uncrossed double dissociation in self-reference recall speed between conscientiousness and openness across the two studies).

For Study 3, I hypothesized that the pattern of inter-correlations examined in Study 2 among measures of elaborative processing and personality would hold for the imaginativeness adjectives encoded in the self-reference task (i.e., slower recall and less false recognition of imaginativeness adjectives would be related to higher direct or indirect measures of the participants' openness and imaginativeness). Furthermore, I hypothesized that higher direct assessments of the participants' best friend's orderliness would be related to a slower rate of approach to asymptote and less false recognition of the orderliness adjectives encoded in the other-reference task. Importantly, participants' direct and indirect assessment of their best friend's orderliness were not expected to be related to the measures of elaborative processing from the self-reference task, nor was it expected that the direct and indirect assessment of their own imaginativeness would be related to the measures of elaborative processing from the other-reference task (i.e., an uncrossed double dissociation among self- and other-reference recall speed and participants' openness and their assessment of their best friend's conscientiousness). These two hypotheses served to establish further divergent validity for the memory-based personality measures (i.e., supporting the idea that congruence between the tapped concept, whether self or other, and the trait is mandatory for the hypothesized relationships to hold). ${ }^{1}$

\footnotetext{
${ }^{1}$ There were additional hypothesis tests that could not be conducted for Study 1, 2, and 3. In particular, the amount of correctly recalled adjectives from the structural, rhyming, and semantic tasks was not high
} 
It was not clear a priori whether the measures of elaborative or organizational processing would be related to one type of personality trait measure (e.g., direct) more than another (e.g., indirect). Therefore, this aspect of the research was considered exploratory, although theoretically these analyses were meaningful for bringing together the personality, social cognition, and memory literatures (e.g., Gawronski \& Bodenhausen's, 2011, APE model and Einstein \& Hunt's, 1980, memory framework that combines research on elaborative and organizational processing). In particular, if the measures of elaborative processing relate to direct personality measures, but not indirect ones, the results would suggest that the item-specific information elicited by selfreference encoding is propositional rather than associative. In contrast, if the measures of elaborative processing are related to indirect personality measures, but not direct ones, the results would suggest that the item-specific information is associative not propositional. Of course, it may be both associative and propositional.

\section{Self-Reported and Observed Behaviour}

Across all three studies, I hypothesized that the measures of elaborative processing following self-reference encoding would predict participants' self-reported and observed trait-related behaviour. In Study 3, I hypothesized that the measures of

enough to adequately fit cumulative recall curves (i.e., Equation 1) to individual participant data, thus the partial correlations among the rate of approach parameter for trait adjectives judged in these task and direct and indirect trait measures were not examined. I hypothesized that these relationships would not be significant (because the encoding task did not require access to the self-concept). Given that recall was inadequate in these tasks, cumulative recall for the combined structural, rhyming, and semantic tasks were compared graphically to the cumulative recall from the self-reference task (i.e., without use of inferential tests). Additionally, the best fitting curve (Equation 1) for cumulative recall performance of the combined tasks was estimated for participants who were relatively low, medium, or high on the trait reflected by the self-referenced adjectives (e.g., low, medium, and high orderliness in Study 1). I only expected slower recall (lower rate of approach) to be related to participants' direct or indirect personality measures (of themselves or their best friend) when a self-reference (or other-reference) judgment was made. 
elaborative processing following other-reference encoding would predict participants' reports of their best friends' trait-related attitudes and behaviours.

\section{Study 1}

\section{Method}

Participants. Ninety-eight undergraduate psychology students were recruited from the Carleton University Experiment Sign-Up System (SONA). Sixty-nine participants were female (70.4\%; one did not report their gender). All participants were included in analyses, except when explicitly stated otherwise.

\section{Materials.}

Levels-of-processing. Forty trait conscientiousness adjectives were taken from De Raad and Peabody's (2005) study. The adjectives were divided into four lists that describe the positive and negative pole of orderliness and the positive and negative pole of impulse control. Table 1 presents the fours lists and Appendix A presents the precise method used to create the lists.

Table 1

Levels-of-Processing Items for the Orderliness and Impulse Control Facets of Conscientiousness

Memory List

\begin{tabular}{cccc} 
Orderly & Controlled & Disorderly & Impulsive \\
\hline Punctual & Cautious & Disorderly & Impulsive \\
Organized & Careful & Negligent & Rash \\
Efficient & Prudent & Careless & Reckless \\
Particular & Deliberate & Lax & Hasty \\
Meticulous & Moderate & Confused & Frivolous \\
Strict & Restrained & Chaotic & Excessive
\end{tabular}


Precise

Accurate

Measured

Prompt
Disciplined

Composed

Serious

Practical
Sloppy

Forgetful

Contradictory

Unpredictable
Insatiable

Restless

Exaggerated

Lavish

Note. Items in bold have been used in previous research on conscientiousness-IATs.

All lists were equated in terms of word frequency, word length, and

meaningfulness. Not surprisingly, the orderly and controlled lists were rated significantly more likeable than the disorderly and impulsive lists, based on the available likeableness norms from Anderson's (1968) list of personality trait adjectives. The lists were equated in terms of their latent semantic association (LSA) to the impulsivity concept. In contrast, the orderly and controlled lists had a stronger LSA to the orderliness concept than to the impulsive list $\left(M_{\mathrm{diff}}=.083, S E=.023, p=.014\right.$ and $\left.M_{\mathrm{diff}}=.11, S E=.032, p=.035\right)$.

Given that the main focus was on differences in recall between the self-reference and semantic encoding tasks (i.e., the orderly and controlled lists), these differences in LSA were not considered important. Overall, the orderly and controlled lists were equated in terms of word frequency, word length, likableness, meaningfulness, impulsivity LSA, and orderliness LSA. Table 2 presents descriptive and inferential statistics for the psycholinguistic properties of the adjective lists.

Table 2

Mean Psycholinguistic Properties for the Orderliness and Impulse Control Memory Lists

\begin{tabular}{cccccc}
\hline & Orderly & Controlled & Disorderly & Impulsive & $F$ \\
\hline Word Frequency & $2.33(.47)$ & $2.42(.79)$ & $2.07(.52)$ & $2.08(.34)$ & 1.01 \\
Word Length & $8.1(1.45)$ & $8.5(1.43)$ & $8.6(3.03)$ & $7.9(2.23)$ & .24 \\
Likeableness $^{\mathrm{a}}$ & $413^{\mathrm{b}}(80.77)$ & $377^{\mathrm{c}}(36.60)$ & $189^{\mathrm{d}}(66.38)$ & $236^{\mathrm{e}}(55.56)$ & $19.22^{* * *}$
\end{tabular}


Meaningfulness $^{\mathrm{a}} \quad 366^{\mathrm{b}}(18.83) \quad 347^{\mathrm{c}}(20.86) \quad 375^{\mathrm{d}}(9.44) \quad 352^{\mathrm{e}}(25.14) \quad 2.64$

$\begin{array}{llllll}\text { Impulsivity LSA } & 0.104(.058) & 0.112(.045) & 0.089(.063) & 0.101(.066) & .26\end{array}$

Orderliness LSA $\quad 0.192(.095) \quad 0.169(.062) \quad 0.121(.052) \quad 0.085(.039) \quad 5.37^{* *}$

Note. Standard deviation is presented within parentheses. LSA = latent semantic association.

${ }^{\mathrm{a}}$ Calculated from Anderson's (1968) personality trait norms. ${ }^{\mathrm{b}}$ missing data for organized, particular, and measured. ${ }^{\mathrm{c}}$ missing data for restrained ${ }^{\mathrm{d}}$ missing data for disorderly, lax, confused, chaotic, and contradictory . ${ }^{\mathrm{e}}$ missing data for hasty, excessive, insatiable, exaggerated, and lavish.

${ }^{* *} p<.01{ }^{* * *} p<.001$.

For trials in the level-of-processing task, an orienting question appeared in the center of the computer screen followed by a trait adjective. The orienting question for the structural encoding task was "Two or more e's?" and the orienting question for the selfreference task was “Describes Me?". The orienting question posed for the rhyming and semantic encoding tasks changed every trial, but followed the template "Rhymes with XXXX?" and "Means the same as XXXX?", where XXXX was a different word each trial. Half of the words that replaced the XXXX for the rhyming task were in fact rhymes or near rhymes of the presented trait adjective taken from various disparate nonpersonality trait categories, and half for the semantic task were synonyms or near synonyms taken from various personality trait categories. Approximately half of the stimuli in the structural task required a "Yes" response (4 out of 10). Half of the questions for the rhyming and semantic task required a "Yes" response. Given the subjective nature of the self-reference task, the number of "Yes" and "No" responses could not be 
controlled. The procedure used to obtain the orienting questions for the rhyming and semantic encoding tasks is described in Appendix B.

Finally, to buffer against the known effect of primacy and recency in free recall (e.g., Glazner \& Cunitz, 1966), different color words were selected for the first four trials (green, cyan, blue, and magenta) and for the last four trials (red, brown, yellow, and orange). Latent semantic analysis showed that these color words were highly associated to one another, but largely unrelated to the word lists.

Working memory. A complex reading span task was administered (Conway et al., 2005). Participants read sentences of 10 to 15 words and indicated whether each sentence made sense or not. In total, 33 sentences were constructed such that changing one noun in the sentence rendered it senseless (e.g., "Just because there's a ring on his finger doesn't mean he's married" vs. "Just because there's a lake on his finger doesn't mean he's married"). In addition, participants were required to remember a capitalized letter that was presented after they made a judgment about a sentence. They were asked to keep track of the letters and the order that the letters were presented. After judging whether two, three, four, five, six, or seven sentences made sense or not, participants were asked to recall the capitalized letters in order. In an effort to reduce extraneous strategies for remembering the letters, the letters presented within each set of sentences did not rhyme and were orthographically dissimilar (e.g. Z and O vs. D and P). Appendix C shows the sentences, the letters, and the exact presentation order.

Recognition memory. In addition to the 40 old conscientiousness adjectives from the levels-of-processing task, 40 new conscientiousness lures and 20 categorically unrelated adjectives were selected for the recognition memory test. The 40 lures were 
selected from the remaining conscientiousness adjectives in the De Raad and Peabody (2005) study. The 20 categorically unrelated items were selected from categories of adjectives describing physical appearance, including height, weight, and strength. Appendix D presents the steps taken in selecting the lures and unrelated stimuli.

Implicit Association Test. The orderliness-IAT was created from the orderly and disorderly memory list adjectives, as well as "Me" (I, self, my, me, and mine) and "Not Me" (they, them, their, theirs, and others) words. The "Me" and "Not Me" labels have been used in recent research conducted by Peters and Gawronkski (2011) and they seem a better match to the question posed in the self-reference task (i.e., "Describes $M e$ ?") than the "Self" and "Others" labels used in the reviewed IAT studies. The exemplars of the "Me" and "Not Me" concepts were selected from Peters and Gawronski's study and they are similar to those used in the reviewed studies. The controlled-IAT was created from the controlled and impulsive memory list adjectives, as well as the "Me" and "Not Me" words. Greenwald, Nosek, and Banji's (2003) twelve-step improved scoring algorithm was used to compute the $D_{3}$ variant of the IAT effect for each participant and separately for the orderliness- and controlled-IATs.

Conscientious behaviour. A d 2 test of concentration and attention (Brickenkamp, 1981; Brickenkamp \& Zillmer, 1998) served as a behavioural measure of conscientiousness. For the standard d2 test (e.g., Bates \& Lemay, 2003), participants are presented a single piece of paper that consists of 14 rows of 47 characters. They are instructed to cross-out each " $d$ " that appears with exactly two marks around it. The characters are "d"s and "p"s with 1 to 4 marks configured above or below the character. Participants are given 20 seconds per line and asked to continue to the next line without 
taking a break. For the present study, a customized self-paced $\mathrm{d} 2$ test was created (Steffens, 2004; Steffens \&Schulze-König, 2006). Nine rows of 47 characters were systematically generated rending exactly a 3 -minute test $(20$ seconds $\mathrm{x} 9$ rows $=180$ seconds). The characters were "d"s and "p"s with 1 to 3 marks positioned in one of four locations around the letter (top left, top right, bottom left, and bottom right), with no more than two marks in one location. Participants were asked to cross-out "d"s that appear with exactly two marks (e.g., d' ), and to ignore all other characters (e.g., ', $\left.\mathrm{p}^{\prime}, \mathrm{p} \mathrm{d}_{1}\right)$. Appendix E presents the customized $\mathrm{d} 2$ test that was administered as well as the details concerning its development. To provide measures of errors committed in the d2 test (Steffens \& Schulze-König, 2006; Steffens, 2004), omission error, commission error, total error, and percent error were calculated following Bates and Lemay (2004).

As further measures of conscientious behaviour, the exact time when participants arrived at the laboratory was recorded to determine the degree of lateness for the experimental session (arrival time minus scheduled time, Back, Schmukle, \& Egloff, 2009). In addition, the week within the semester and the time of day were also recorded (Aviv, Zelenski, Rallo, \& Larsen, 2002; Zelenski, Rusting, \& Larsen, 2003). As all these variables were incidental to the experiment, they were taken outside of the experimental procedure (except for lateness, which was recorded shortly after participants' arrival, to ensure accurate measurement). Hence, participants' awareness that these variables were measured did not affect the resultant measurements.

Personality self-report. A questionnaire with items taken from the International Personality Item Pool (IPIP; Goldberg et al. 2006) provided a measure of the Big Five and its facets that have been validated against Costa and McCrae's (1992) NEO- 
Personality-Inventory-Revised (NEO-PI-R). The 10-item orderliness facet scale $(\alpha=.83)$ of conscientiousness and the 10-item immoderation facet scale of neuroticism $(\alpha=.78)$ were selected as direct measures of the orderliness and impulse control facets. ${ }^{2}$ In addition, two 10-item scales measuring broader conscientiousness $(\alpha=.86)$ and neuroticism $(\alpha=.84)$ were included. As some studies have suggested that extraversion is related to better incidental recall of adjectives (and nouns) with positive valence (e.g., Rusting \& Larsen, 1998; Zelenski, 2007), a 10-item measure of extraversion $(\alpha=.88)$ was included. Finally, to examine divergent validity and to include a wider variety of items than the particular ones of interest in the present study, a 10-item facet scale of openness $(\alpha=.75)$ was included. Appendix F presents the 60 -item personality questionnaire.

Behaviour self-report. A second questionnaire assessed various self-reported behaviours. The questionnaire consisted of a subset of items from the Behavior Report Form (BRF; Paunonen, 1998, 2003). All items from the BRF that were shown to have replicable relationships with conscientiousness, neuroticism, extraversion, or openness as measured by the NEO-PI-R were selected for the present study. In addition, a measure of conscientious behaviour from one of the reviewed studies (Schmukle, Back, and Egloff, 2008) was included. Specifically, a single item asked how frequently participants clean the inside of their windows at home each year. In addition, a question asked whether participants enjoy reading classic literature. Schmukle, Back, and Egloff (2008) found that this item relates to direct and indirect measures of openness to experience. Appendix G presents the 20-item self-reported behaviour questionnaire.

\footnotetext{
${ }^{2}$ Although impulse control appears as a facet of conscientiousness in the De Raad and Peabody (2005) study, some researchers have found that its items load more highly onto neuroticism (e.g., McCrea \& Costa, 1997).
} 
Design. The levels-of-processing task was a one-way (encoding task: structural, rhyming, semantic, self-reference) repeated-measures design. When recall was examined, the design became factorial with recall trial (first and second) as an additional repeatedmeasures variable. As the primary goal of the present study was to examine individual differences, participants received the exact same order of presentation of tasks and items within tasks (Grumm \& Collani, 2007; Steffens \& Schulze-König, 2006; Banse, Seise, \& Zerbes, 2001). Researchers have argued that changing procedural details across participants introduces error variance and decreases the power to detect correlations (Banse, Seise, \& Zerbes, 2001; Steffens \& Schulze-König, 2006). Furthermore, Zelenski (2007) argued that fixing the order of presentation of items and tasks in research on individual differences might raise questions concerning the generalizability of results, but not the validity of observed individual differences (see also Gawronski, 2002). Therefore, the experimental procedure reflects the static order of presentation of tasks and items within tasks. When randomization was used, participants underwent the same randomized order.

Procedure. Prior to participants' arrival, they were assigned a numeric ID number that uniquely identified their data on the computer tasks and questionnaires, without containing any personal information (e.g., their name). When they arrived, they were immediately escorted to a small testing room (6111B HCI or 6111C HCI). While participants provided informed consent, the researcher recorded their arrival time on a sheet with their ID number. Once participants provided consent, the researcher explained that there would be a series of cognitive tasks administered on the computer or with paper and pen. It was further explained that each task had a set of standardized instructions that 
everyone would see. The researcher's involvement would therefore be to guide participants through the experiment by starting the computerized tasks and providing the paper-and-pen tasks. The reason for the researcher's minimal involvement was to avoid additional sources of variation (error variance) introduced by their differential involvement in the procedure across participants. Nevertheless, participants were encouraged to ask questions if they needed clarification of the instructions.

The levels-of-processing task was administered first on a computer. To create an incidental learning situation typical of SRE experiments (e.g., Klein \& Kilhstrom, 1986; Rogers et al., 1977), participants were asked to answer the orienting questions as quickly and accurately as possible with no mention that there would be a memory test. The judgments required a yes or no response, which was made by pressing the button labeled "YES" on the far left of the response pad or the button labeled "NO" on the far right. Given that some of the words were relatively infrequent in the English language, participants were asked to press the button labeled "UNSURE" in the middle of the response pad, if they were completely unsure. They were shown on the computer screen the four orienting questions along with one example for clarity ("If the question is "Two or more e's?" and the word that appears is "teal", you should press the 'NO' key"). Furthermore, they were asked to respond "YES" if the presented word mostly rhymes or mostly means the same as the word presented in the rhyming and semantic orienting questions respectively.

Trials proceeded in the same fashion as described in Rogers et al.'s (1977) seminal study. Specifically, an orienting question specific to the encoding task was displayed for $3000 \mathrm{~ms}$ proceeded by a blank screen for $500 \mathrm{~ms}$. Then, a trait adjective 
appeared and it did not disappear until the participant pressed one of the three response buttons. Finally, a $2000 \mathrm{~ms}$ inter-trial interval elapsed before the next orienting question appeared. Response time and the selected response were recorded.

The first four trials consisted of the color words selected to buffer against primacy, where each color word was paired with one of the four encoding tasks, allowing participants to practice each task once before starting the critical trials. The 40 trait adjectives were then presented, starting with the 10 adjectives from the impulsive list presented after the structural orienting question (i.e., "Two or more e's?"). Next, the 10 adjectives from the disorderly list were presented after the rhyming orienting question (e.g., "Rhymes with Quarterly?"), followed by the 10 adjectives from the controlled list presented after the semantic orienting question (e.g., "Means the same as Guarded?"). The last 10 adjectives were from the orderly list presented after the self-reference orienting question (i.e., "Describes me?"). Finally, the four color words selected to buffer against recency were presented in the same manner as the four color words presented at the beginning of the task. The order of presentation of the adjectives within each encoding task was randomized. The assignment of a yes or no response to both the rhyming and semantic encoding tasks was quasi-randomized, under the constraint that half required a yes response. Appendix H presents the 48 trials with the assigned encoding task, stimuli, and correct responses.

Following the levels-of-processing task, participants were handed the $\mathrm{d} 2$ test. The instructions asked them to cross out all characters that appeared as a "d" with exactly two primes, and to ignore all other characters. Examples of characters they should and should not cross-out were presented. They were asked to start on the left-hand side of the first 
line and continue to the right, from top to bottom. After 3 minutes timed using a stopwatch, the researcher called out "Stop" and asked participants to circle the last character they looked at. The $\mathrm{d} 2$ test sheets were then collected.

For the first free recall task, participants were handed a sheet of paper with instructions and 24 numbered lines (see Appendix I). The instructions told participants "this is a surprise memory test" and asked them to recall as many words as possible that were presented in the judgment task and to write them down one-at-a-time on the sheet, starting on the line numbered "1.". Every minute the researcher called out "Now", and participants placed a slash “/” beside the last recalled word. After 5 minutes timed using a stopwatch, the sheets were collected. After a brief pause, participants were handed a second free recall sheet, much like the first one (see Appendix J). The instructions were the same; except participants were asked to recall the words they listed in the first recall task, as well as new ones. They were given another 5 minutes, with the researcher calling out "Now" every minute.

Following the second recall task, participants engaged in a computerized complex reading span task (Conway et al., 2005). Here, participants were asked to read sentences of 10 to 15 words and indicate as quickly and accurately as possible whether the sentence makes sense. Once participants responded using the "YES" or "NO" response button, a capitalized letter appeared for $1000 \mathrm{~ms}$. After a varying number of sentences, participants were asked to recall the capitalized letters in order. There were two sets of practice trials: The first set contained three sentences with only the yes-no judgment, and the next set contained three sentences with the yes-no judgment and capitalized letter, followed by a recall trial. After the practice trials, there were 27 sentences and 6 recall trials. 
Specifically, there was a recall trial after reading sets of two, three, four, five, six and seven sentences in that order. Participants were not told how many sentences there would be in a set. Participants were given as much time as they required to type the letters they saw in order, using the keyboard and terminating the recall trial by pressing the "Return" key (Unsworth, Redick, Heitz, Broadway, \& Engle, 2009 used a similar procedure).

Participants then completed a computerized recognition test. The instructions explained there would be a series of words presented one-at-a-time and their task was to indicate whether the word was presented in the judgment task completed at the beginning of the experiment. The 100 recognition memory items were presented in one randomized order. Participants were instructed to press the "YES" button when they believe the item was presented in the judgment task or press the "NO" button when they believe the item was not presented in that task. There was a $500 \mathrm{~ms}$ inter-stimulus-interval.

Following the recognition test, the IATs were administered. The initial set of instructions explained there would be a series of 13 short categorization tasks. Before each task, a new set of instructions for the next task appeared. The instructions clearly emphasized that speed of categorization was most important, and it would be okay to make some mistakes due to fast responding. If mistakes were made, they were shown an error message in red that asked them to redo the trial. The IATs consisted of the typical seven-block structure (Greenwald, McGhee, \& Schwartz, 1998; Greenwald, Nosek, Banaji, 2003), and the two IATs were completed in a relatively seamless way with appropriate pauses for instructions (Grumm \& Collani, 2007; Schmukle, Back, \& Egloff, 2008). The exact order of the IAT blocks and the number of trials within each block is presented in Table 3. The category labels associated with the left and right response 
buttons were displayed at the top left and top right of the screen, as a reminder of the category-response key associations for the current task.

Table 3

Structure of the Orderliness and Controlled-IATs

\begin{tabular}{ccccc}
\hline Block & Task & Trials & Left key & Right key \\
\hline 1 & Target Discrimination & 20 & Me & Not Me \\
2 & Attribute Discrimination & 40 & Orderly & Disorderly \\
3 & Combined Discrimination_1 & 40 & $\mathrm{Me}+$ Orderly & Not Me + Disorderly \\
4 & Combined Discrimination_2 & 80 & $\mathrm{Me}+$ Orderly & Not Me + Disorderly \\
\multicolumn{5}{c}{ Reverse Attribute } \\
5 & Discrimination & 40 & Disorderly & Orderly \\
6 & Combined Discrimination_3 & 40 & $\mathrm{Me}+$ Disorderly & Not Me + Orderly \\
7 & Combined Discrimination_4 & 80 & $\mathrm{Me}+$ Disorderly & Not Me + Orderly \\
8 & Attribute discrimination & 40 & Impulsive & Controlled \\
9 & Combined Discrimination_1 & 40 & $\mathrm{Me}+$ Impulsive & Not Me + Controlled \\
10 & Combined Discrimination_2 & 80 & $\mathrm{Me}+$ Impulsive & Not Me + Controlled \\
& Reverse Attribute & & & Impulsive \\
11 & Discrimination & 40 & Controlled & Not Me + Impulsive \\
12 & Combined Discrimination_3 & 40 & $\mathrm{Me}+$ Controlled & Not Me + Impulsive \\
\hline 13 & Combined Discrimination_4 & 80 & $\mathrm{Me}+$ Controlled & Non
\end{tabular}

The first block consisted of two presentations of the "Me" and "Not Me" words for a total of 20 trials. Participants categorized "Me" words using the left response button (labeled "YES") and "Not Me" words using the right response button (labeled "NO").

The second block consisted of two presentations of the orderly and disorderly adjectives for a total of 40 trials. Participants pressed the left response key for accurate categorization of the orderly words and the right response key for the disorderly words. The third block consisted of 40 trials and was a combination of blocks 1 and 2 (with only 
one presentation of the words from block 2). The same key assignments learned in blocks one and two were implemented. Note that there were twice as many "Me" and "Not Me" words to equate the number of "Me" and "Not Me" versus "Orderly" and "Disorderly" categorizations in a combined block, which was true for all subsequent combined blocks. The fourth block was identical to the third block, except that all items were presented twice for a total of 80 trials. The fifth block was the exact same as the second block except that the category-response key assignments were reversed, such that participants were required to press the left response key for accurate categorization of the "Disorderly" words and the right response key for "Orderly" words. The sixth and seventh blocks were the exact same as the third and fourth blocks, except that the new category-response key assignment learned in the fifth block persisted throughout. Finally, blocks eight through thirteen followed the same pattern of category-response key assignments as blocks two through seven, except that the "Controlled" and "Impulsive" words replaced the "Orderly" and "Disorderly" words, respectively. In total, there were 660 trials.

IAT trials proceeded as follows. Stimuli were presented in black lower case letters in the center of the computer screen against a white background. The categorization response options associated with the left and right response keys (e.g., "Me "and "Not Me" in block 1) appeared at the top left and top right of the screen respectively. When a combined discrimination block was presented (e.g., block 3), the category labels assigned to the left response key appeared at the top left of the screen and the category labels assigned to the right response key appeared at the top right of the screen. The category labels remained on the screen until the trial was complete, which occurred when 
participants pressed one of the two response keys. There was a $250 \mathrm{~ms}$ inter-trial interval. If an error was made, participants were presented a screen stating "Error, please wait and then try again" in red in the center of the screen for 3 seconds, after which the trial was repeated. A single randomized order of trials within each block was implemented. When stimuli were presented twice within a block (e.g., blocks 4, 7, 10, and 13), the randomized order for the first presentation of stimuli was duplicated for the second presentation. $^{3}$

Once the IAT was completed, participants were escorted to an empty conference room $(6111 \mathrm{E} \mathrm{HCI})$ where they were presented the self-report personality questionnaire. They were told explicitly that the questionnaire was designed to assess their personality. The questionnaire was completed in a different context than the computerized tasks, in an effort to minimize potential transfer effects of the memory and IAT tasks on self-reported personality. Once participants completed the personality questionnaire, they were presented the behaviour report questionnaire. The instructions asked participants to describe themselves by answering the questions as accurately as possible. The items within the personality questionnaire were randomized while the items in the behaviour questionnaire were quasi-randomized, under the constraint that items with a similar response format were grouped. Finally, participants were debriefed, thanked, and granted course credit. The average completion time was 76 minutes $(S D=7$ minutes $)$.

\footnotetext{
${ }^{3}$ Two related technical issues with the IAT were experienced throughout the duration of data collection. First, the program (SuperLab) froze at some point during the IAT for nine participants $(9.2 \%)$. In the event of a crash, the program was restarted at the beginning of the block of trials where the program crashed. The first crash happened for the fifth participant. Thereafter, participants were told that if a blank screen appeared during the task and the response buttons would not allow the program to continue, they would need to retrieve the experimenter. Second, it was brought to the researchers' attention that occasionally blank screens would appear between trials, which required participants to press a response key to proceed to the next trial. Thereafter, participants were told that if they experienced that problem, they would need to press one of the response keys to continue. This issue generally only persisted for one block of trials, but it did occur more than once within the procedure for some participants.
} 


\section{Results}

Self-reference effect. A series of one-way (encoding task: structural, rhyming, semantic, self-reference) repeated-measures analyses of variance (ANOVAs) were conducted on response time (RT) and accuracy (proportion correct) for the judgments made in the levels-of-processing task. Proportion recalled following the levels-ofprocessing task was submitted to a 4(encoding task: structural, rhyming, semantic, selfreference) x 2(recall trial: first, second) repeated-measures ANOVA. Item gain ${ }^{4}$ and item $\operatorname{loss}^{5}$ was submitted to separate one-way (encoding task: structural, rhyming, semantic, self-reference) repeated-measures ANOVAs. Proportion of correct recognition was submitted to one-way (encoding task: structural, rhyming, semantic, self-reference) repeated-measures ANOVA. Lastly, proportion of yes responses in the recognition test was submitted to one-way (item type: old, lure, unrelated) repeated-measures ANOVA. All degrees of freedom were based on the Greenhouse-Geisser adjustment for violation of the assumption of sphericity.

The ANOVA on RTs revealed a significant main effect of encoding task, $F(2.34$, $224.21)=39.15, \eta^{2}=.29, p<.001$. The structural $(M=1,150 \mathrm{~ms}, S E=59 \mathrm{~ms})$ and rhyming $(M=1,125 \mathrm{~ms}, S E=33 \mathrm{~ms})$ tasks were executed faster than the semantic $(M=$ $1,554 \mathrm{~ms} S E=49 \mathrm{~ms})$ and self-reference $(M=1,594 \mathrm{~ms} S E=63 \mathrm{~ms})$ tasks, $M_{\mathrm{diff}}=-404$

\footnotetext{
${ }^{4}$ Item gain was calculated as the proportion of items that were not recalled on the first recall trial but were then recalled on the second recall trial (i.e., the denominator was 10, reflecting the total number of list items). For example, if a participant recalled two self-referenced items on the first recall trial, and then recalled two new items on the second trial, item gain was $2 / 10=.20$.

${ }^{5}$ Item loss was calculated as the proportion of items that were recalled during the first recall trial and that were not recalled on the second recall trial. Calculation of item loss in this manner takes into account differences in the overall level of recall between conditions (Burns, 1993). One issue with the item loss measure is that participants who failed to recall an item by the end of the first recall task technically could not lose an item in the second recall trial (i.e., item loss was impossible). Results were calculated in two ways: 1) by inputting a score of zero in this case and 2) by excluding participants that did not recall an adjective by the end of the first recall trial.
} 
$\mathrm{ms}, S E=69 \mathrm{~ms}, p<.001$ and $M_{\mathrm{diff}}=-445 \mathrm{~ms}, S E=69 \mathrm{~ms}, p<.001$ respectively. All other pairwise comparison were not statistically significant, $p \mathrm{~s}>.50$. Hence, as is typically observed (Craik \& Tulving, 1975), the deeper processing tasks took longer to complete than the shallow ones. Of particular note is that the decisions made in the selfreference and semantic tasks took an equivalent amount of time to complete.

For the ANOVA on accuracy, the self-reference task was excluded because there was no basis with which to judge the accuracy of self-reference responses. The main effect of encoding task was significant, $F(1.57,150.21)=132.62, \eta^{2}=.58, \mathrm{p}<.001$. The responses provided in the structural $(M=.97, S E=.007)$ and rhyming $(M=.96, S E=$ $.007)$ tasks were more accurate than those provided in the semantic $(M=.77 S E=.014)$ task, $M_{\text {diff }}=.20, S E=.016, p<.001$ and $M_{\text {diff }}=.19, S E=.015, p<.001$ respectively. All other pairwise comparisons were not statistically significant, $p \mathrm{~s}>.50$. To check whether the incorrect responses in the semantic task were due to selecting the unsure response option more often, the analysis was rerun after deleting all unsure responses $(1.8 \%)$. The main effect of encoding task was nearly eliminated, $F(1.59,192)=3.97, \eta^{2}=.04, p=$ .029. The responses provided in the rhyming task $(M=.98, S E=.008)$ were marginally more accurate than those provided in the semantic task $(M=.95, S E=.009), M_{\text {diff }}=.031$, $S E=.013, p=.054$. All other pairwise comparisons were not statistically significant, $p \mathrm{~s}>$ .50. Hence, aside from participants selecting unsure responses more often in the semantic task (some of the synonym judgments may have been ambiguous), participants performed the encoding tasks very accurately.

Analysis of proportion recalled uncovered a significant main effect of encoding task, $F(1.87,169.28)=400.03, \eta^{2}=.81, p<.001$, representing a strong levels-of- 
processing effect. The self-reference task yielded higher recall $(M=.38, S E=.015)$ than did the semantic $(M=.07, S E=.007)$, rhyming $(M=.02, S E=.005)$, and structural $(M=$ $.04, S E=.007)$ tasks, $p \mathrm{~s}<.001$. In turn, the semantic task yielded higher recall than did the rhyming and structural tasks, $p \mathrm{~s}<.01$. Finally, the structural task yielded higher recall than did the rhyming task, $p<.01$. The main effect of recall trial was marginally significant, $F(1,96)=3.63, \eta^{2}=.036, p=.06$, indicating that participants tended to recall more items on the second recall trial than the first trial (i.e., hyperamnesia; more item gains than item losses, e.g., Klein, et al., 1989). Finally, the Encoding Task x Recall Trial interaction was not significant, $F(1.76,169.28)=.69, \eta^{2}=.007, p=.49$. Other than replicating the levels-of-processing effect, these data reveal that the semantic and shallow encoding tasks produced very poor recall (less than one adjective on average for each task). Therefore, for the present study, cumulative recall curve parameters were only examined for self-referenced adjectives. Figure 2 presents cumulative recall as a function of encoding task. 


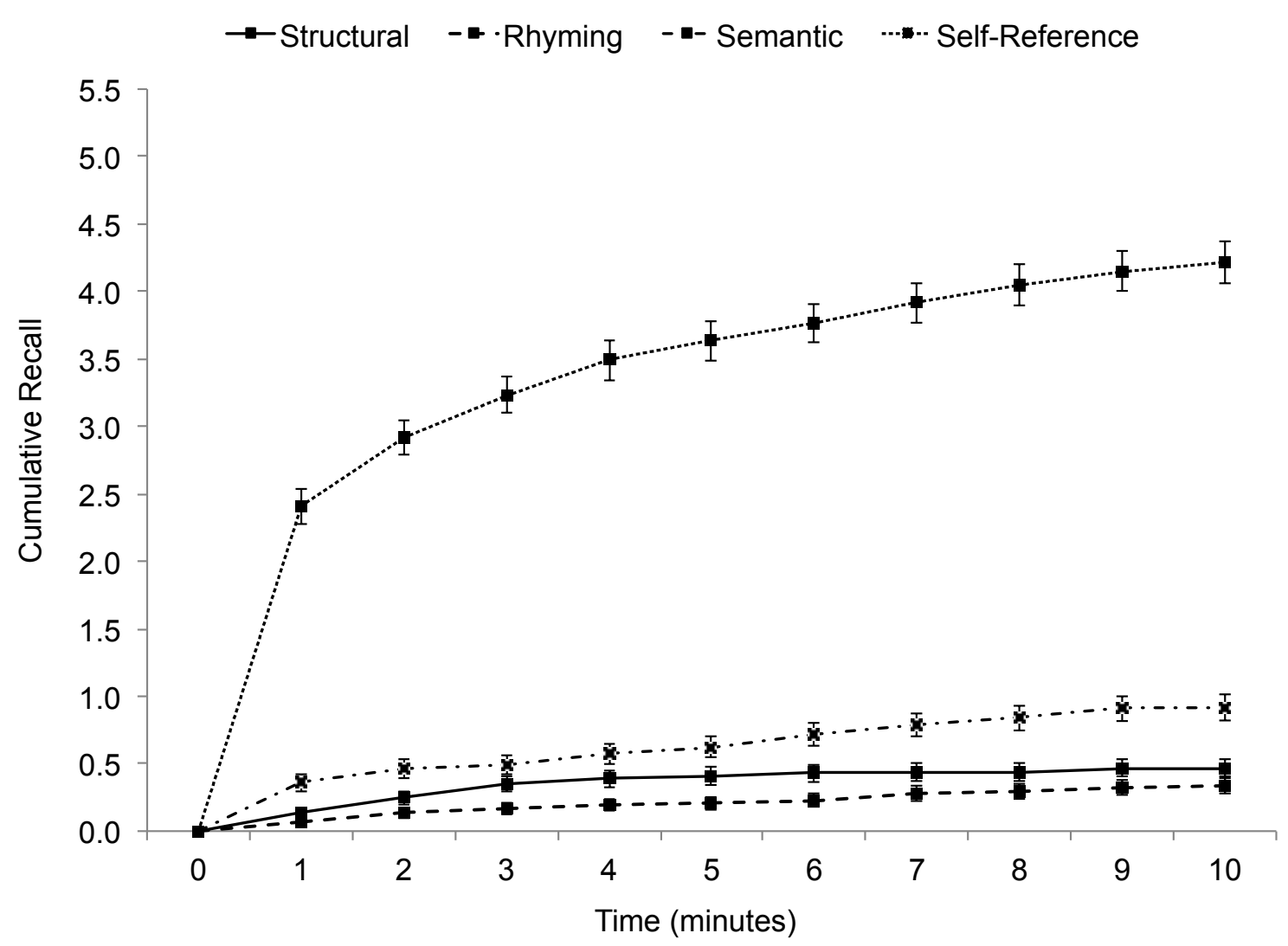

Figure 2. Cumulative recall in Study 1 as a function of encoding task. Error bars represent standard error of the mean.

For the ANOVA on item gain, there was a significant main effect of encoding task, $F(1.79,171.58)=22.12, \eta^{2}=.19, p<.001$. As was expected, item gain was higher following self-reference $(M=.066, S E=.009)$ than semantic $(M=.029, S E=.005)$, rhyming $(M=.011, S E=.003)$, and structural $(M=.007, S E=.003)$ encoding, $p \mathrm{~s}<.01$. Item gain was also higher following semantic than rhyming and structural encoding, $p \mathrm{~s}<$ .05 . Finally, item gain did not differ between the structural and rhyming tasks, $p=.35$. These results suggest that the self-reference task lead to more elaborative processing than did the other encoding tasks.

The ANOVA on item loss revealed a statistically significant main effect of encoding task, $F(2.32,222.83)=6.07, \eta^{2}=.06, p=.002$. Follow-up comparisons 
showed that the proportion of item loss was significantly lower following structural $(M=$ $.04, S E=.02)$ encoding than it was for semantic $(M=.14, S E=.03)$ and self-reference $(M=.12, S E=.02)$ encoding, $M_{\text {diff }}=.10, S E=.04, p=.004$ and $M_{\text {diff }}=.08, S E=.03, p=$ .006 , respectively. Furthermore, item loss was significantly lower following rhyming $(M$ $=.04, S E=.02)$ encoding than semantic and self-reference encoding, $M_{\mathrm{diff}}=.11, S E=$ $.04, p=.006$ and $M_{\text {diff }}=.09, S E=.02, p<.001$, respectively. Although the semantic task elicited more item loss than did the self-reference task, the difference did not reach statistical significance, $M_{\text {diff }}=.02, S E=.04, p=.60$. However, it should be noted that the rate of item loss in the structural, rhyming, and semantic tasks was artificially reduced by the number of participants who did not recall any of the adjectives presented in theses tasks (making item loss impossible). Specifically, there were 65 participants (66.3\%) who did not recall any structurally encoded adjectives by the end of the first recall task, 82 participants $(83.7 \%)$ who did not recall any for the rhyming task, and 52 participants $(53.1 \%)$ who did not recall any for the semantic task, whereas there was only 1 participant $(0.1 \%)$ who did not recall any for the self-reference task. The rate of item loss after excluding theses participants in each task increased for the structural $(M=.13)$, rhyming $(M=.23)$, and semantic $(M=.31)$ tasks, but not for the self-reference task $(M=$ .12), which had the nominally lowest rate of item loss, as was expected. An additional test comparing the rate of item loss between the semantic and self-reference tasks among the 45 participants $(45.9 \%)$ who recalled adjectives encoded in both tasks by the end of the first recall trial revealed significantly more item loss in the semantic task than the self-reference task $(M=.31, S E=.06$ vs. $M=.15, S E=.03), F(1,44)=5.97, \eta^{2}=.12, p$ $=.02$. These results suggest that the self-reference task elicited more organizational 
processing than did the semantic task, confirming that the self-reference effect was in part due to organizational processing. Given the very low proportion recalled following semantic, rhyming, and structural encoding, the item loss data for these tasks are subject to a floor effect. As such, these data must be interpreted with caution. Nevertheless, given the relatively high proportion recalled in the self-reference task by the end of the first recall trial, individual differences in item loss for self-referenced adjectives provided a measure of participants' engagement in organizational processing during self-reference encoding (Klein et al., 1994), which is presented in the next section.

The main effect of encoding task on correct recognition of the levels-ofprocessing items was statistically significant, $F(2.68,254.60)=164.83, \eta^{2}=.63, p<$ .001 , presenting a strong levels-of-processing effect. Specifically, trait adjectives subjected to self-reference encoding $(M=.88, S E=.013)$ were better recognized as having been presented in the levels-of-processing task than were those encoded in the semantic $(M=.74, S E=.016)$, rhyming $(M=.48, S E=.021)$, and structural $(M=.53, S E$ $=.020)$ tasks, $p \mathrm{~s}<.001$. In turn, the trait adjectives presented in the semantic task were better recognized than those presented in the rhyming and structural tasks, $p \mathrm{~s}<.001$. Lastly, the trait adjectives presented in the structural task were better recognized than those presented in the rhyming task, $p=.013$, both of which were close to change accuracy (i.e., .50 correct). This pattern of data mirrors the free recall results.

Lastly, the main effect of item type on proportion of yes responses in the recognition task was significant, $F(1.82,172.99)=1262.29, \eta^{2}=.93, p<.001$. Participants responded yes to unrelated items $(M=.06, S E=.006)$ less often than to lures $(M=.23, S E=.011)$ and old items $(M=.66, S E=.013), p s<.001$. In turn, they 
responded yes to lures on average less often than to old items, $p<.001$. The difference between the unrelated items and the lures suggests significant false recognition of the conscientiousness adjectives that were not presented in the levels-of-processing task. Individual differences in this tendency will be examined in the next section as an additional measure of item-specific processing (e.g., Einstein \& Hunt, 1980; McCabe et al., 2004).

In sum, the memory results showed that participants completed the levels-ofprocessing task accurately, but that some of the synonym judgments in the semantic task may have been ambiguous. Indeed, although Rogers et al. (1977; Experiment 1) reported similar proportions of recall as that observed in the present study for the structural $(M=$ .03 vs. $M=.02)$ and rhyming $(M=.07$ vs. $M=.04)$ tasks, recall was almost double for the semantic task $(M=.13$ vs. $M=.07)$. Nevertheless, the results confirmed a strong levels-of-processing effect on recall and recognition, with self-reference rendering higher recall and correct recognition than semantic encoding, and semantic encoding rendering higher recall and correct recognition than the structural and rhyming tasks (replicating the free recall results of Rogers et al., 1977). Furthermore, item gain was significantly higher and item loss was significantly lower in the self-reference task than it was in the semantic task, suggesting elaborative and organizational processing contributed to the selfreference effect (Symons \& Johnson, 1997). ${ }^{6}$

\footnotetext{
${ }^{6}$ Although Klein et al. (1994, Experiment 1) concluded in their study that the self-reference effect was due to better organizational processing, as evidenced by fewer item losses in a second recall trial, they reported a marginal increase in item gain following self-reference encoding relative to semantic encoding. Given that their experiment only included 20 participants, a firm conclusion that elaborative processing did not influence recall was not warranted as there was not enough power (.37) to detect even a medium effect size (Cohen's $f=.25$ ) at $\alpha=.05$.
} 
Personality and self-reference. Partial correlations controlling for working memory capacity were computed to address the hypotheses concerning the measures of elaborative and organizational processing after making self-reference judgments (Appendix K presents the zero-order correlations). Normality of the distributions was examined for conscientiousness, orderliness, number of yes responses in the selfreference task (labeled encoding-orderliness), rate of approach to asymptote, amount recalled (labeled orderliness recall), correct recognition, false recognition, orderlinessIAT, item gain, item loss, and working memory capacity. Because the sample size was fairly large, the absolute value of skewness and kurtosis and a visual inspection of the distributions were the key determinants for contemplating a transformation or elimination of outliers (Tabachnick \& Fidell, 2007). The working memory capacity distribution was reflect-inverse transformed and two outliers were excluded $(z<-3.00)$. The distribution for the estimated rate of approach to asymptote parameter from the best fitting cumulative recall curve $^{7}$ (i.e., the $\lambda$ in Equation 1) was $\log$ transformed and two outliers were excluded $(z<-3.30)$. Finally, the distributions of item gain and item loss for selfreferenced adjectives were severely positively skewed (reflecting a low level of item gains and losses), but were improved by an inverse transformation. All other distributions were approximately normal without outliers. In the following analyses, the transformed variables were examined and outliers were only excluded when the respective variable was involved in a particular partial correlation. Due to technical error, five cases had

\footnotetext{
${ }^{7}$ The curve fitting toolbox in MATLAB was used to fit Equation 1 to the cumulative recall data for each participant. Specifically, the parameters in Equation 1 that best accounted for the number of unique selfreferenced adjectives recalled by minute $0,1,2,3,4,5,6,7,8,9$, and 10 was estimated for each participant. Generally, the best fitting parameters accounted for most of the variation in the cumulative recall data $\left(R^{2}>.90\right)$, suggesting that indeed the exponential cumulative recall function could successfully account for the cumulative recall data in the present study.
} 
missing data for the orderliness-IAT, one case had missing data for encoding-orderliness, and one case had missing data for the recognition task. As a result, the degrees of freedom varied from 86 to 92 across the following analyses.

Table 4 below displays a partial correlation matrix with the direct and indirect personality measures as well as the elaborative and organizational processing measures for self-referenced orderliness adjectives, controlling for working memory capacity. ${ }^{8}$ As one would expect, the direct measure of conscientiousness and orderliness derived from self-report ratings of dispositional phrases were most highly correlated. Additionally, the self-reference judgments made regarding the orderliness adjectives in the levels-ofprocessing task (i.e., encoding-orderliness) also correlated fairly highly with the direct measures of conscientiousness and orderliness, suggesting a similar source of knowledge was tapped when making personality judgments for all three measures (i.e., personality self-concept was tapped during self-reference encoding). It is noteworthy that McCrea and Costa (1987) reported similar correlation sizes among the same factors derived from ratings of trait adjectives and dispositional phrases.

\footnotetext{
${ }^{8}$ The two substantive differences between the zero-order and partial correlation matrices are the improved relationship between encoding-orderliness and the rate of approach $(r=-.14$ to $r=-.26)$ and a weakened relationship between inversed item gain and the rate of approach $(r=.58$ to $r=.41)$.
} 
Table 4

Partial Correlations Among Direct and Indirect Measures of Conscientiousness and Self-Reference Memory Processes in Study 1

\begin{tabular}{|c|c|c|c|c|c|c|c|c|c|c|}
\hline Measure & 1 & 2 & 3 & 4 & 5 & 6 & 7 & 8 & $9^{\mathrm{a}}$ & $10^{\mathrm{a}}$ \\
\hline 1. Conscientiousness & - & & & & & & & & & \\
\hline 2. Orderliness & $.64^{* * *}$ & - & & & & & & & & \\
\hline 3. Encoding-Orderliness ${ }^{\text {a }}$ & $.45^{* * *}$ & $.43^{* * *}$ & - & & & & & & & \\
\hline 4. Orderliness Recall & .10 & .02 & .02 & - & & & & & & \\
\hline 5. Rate of Approach & $-.22^{*}$ & $-.23^{*}$ & $-.26^{*}$ & $-.34^{* * *}$ & - & & & & & \\
\hline 6. Correct Recognition & .05 & -.05 & .00 & $.41^{* * *}$ & .05 & - & & & & \\
\hline 7. False Recognition & $-.14^{++}$ & -.11 & -.13 & -.05 & .13 & .11 & - & & & \\
\hline 8. Orderliness-IAT & $.19^{+}$ & $.16^{++}$ & $.22^{*}$ & .01 & -.12 & $-.24^{*}$ & $-.17^{++}$ & - & & \\
\hline 9. Inverse Item gain ${ }^{b}$ & -.07 & -.05 & .06 & $-.39^{* * *}$ & $.41^{* * *}$ & -.07 & -.04 & .05 & - & \\
\hline 10. Inverse Item loss ${ }^{b}$ & .04 & .03 & .05 & $.31^{* *}$ & -.01 & .02 & .01 & -.03 & $-.17^{++}$ & - \\
\hline
\end{tabular}

Note. Controlling for working memory capacity. $d f \mathrm{~s}=85-94$

${ }^{a}$ Unsure responses were coded as No ${ }^{b}$ Inverse transformed. Must be interpreted accordingly.

${ }^{++} p<.20 .{ }^{+} p<.10 .{ }^{*} p<.05 .{ }^{* *} p<.01 .{ }^{* * *} p<.001$. 
Of particular interest in the present study were the significant negative partial correlations between the rate of approach parameter and the direct measures of conscientiousness, orderliness, and encoding-orderliness. As hypothesized, individuals who were highly conscientious or orderly recalled the self-referenced orderliness adjectives more slowly over the 10 minutes of recall than did their counterparts. Correct recognition of the self-referenced adjectives was not associated with the direct measures or the rate of approach parameter. However, as one would expect, higher correct recognition was significantly associated with higher orderliness recall. The data on false recognition provided some converging evidence that highly orderly individuals encoded more item-specific information when they related the orderliness adjectives to the selfconcept, but the consistent negative correlations between false recognition and the direct personality measures did not reach a conventional level of statistical significance, nor did the positive correlation between the rate of approach and false recognition (which one would expect to be significant if both measures were tapping item-specific processing). The indirect measure of orderliness (orderliness-IAT) was positively related to the three direct measures, except the relationship only reached a conventional level of statistical significance for the encoding-orderliness measure (the adjectives which comprised the IAT stimuli). Interestingly, the orderliness-IAT was significantly negatively related to correct recognition, suggesting that stronger self-orderliness associations were related to worse performance in recognizing previously self-referenced orderliness adjectives. Curiously, false recognition also tended to be negatively related to orderliness-IAT effects, suggesting that stronger self-orderliness associations in memory were related to 
better rejection of orderliness lures in the recognition task (and better rejection was hypothesized to be due to item-specific information).

The final two rows of Table 4 present the item gain and item loss partial correlations. Item gain and item loss were not associated with the direct or indirect personality measures. Higher item gain was associated with a slower rate of approach to asymptote. This relationship was expected because the rate of approach is a more precise measure of item gain, which uses information from all 10 minutes of recall as opposed to only one datum representing the number of unique items gained in the second recall task (i.e., minutes 6 though 10). Participants who gained more items in the second recall task also recalled significantly more self-referenced orderliness adjectives, suggesting that item-specific information was driving recall. In terms of item loss, participants who forgot fewer self-referenced items in the second recall task recalled significantly more orderliness adjectives, suggesting that relational information was also driving recall. Importantly, the null relationships between item loss and rate of approach, false recognition, and direct personality measures suggest that organizational processing was not driving the negative relationship between the direct personality measures and the memory-based ones. In particular, if a shallower rate of approach was due to poor organization of the material encoded under self-reference among highly conscientious and orderly individuals, item loss would have been positively related to the rate of approach parameter and to the direct personality measures (i.e., more item losses would have been related to shallower curves and higher orderliness), both of which were not the case. 
Personality and cumulative recall. Figure 3 below plots cumulative recall for participants who were low $(n=33)$, medium $(n=33)$, or high $(n=27)$ on the direct measure of orderliness, as defined by approximate tertiles, with the first tertile representing the low orderliness group. The three cumulative recall curves at the top of Figure 3 are approximately equal from the minute 6 onwards (i.e., when the second recall trial started), showing why the item gain measure was not related to orderliness (i.e., the personality differences in recall speed were mostly observed in the first recall task). In particular, the largest difference in cumulative recall occurred at minutes 1 and 2 . This pattern of cumulative recall could be obtained either by the high orderliness group processing less relational information than their counterparts (i.e., which would slow down recall early in the first task, due to a less effective retrieval plan or limited relational information to narrow the search for item-specific cues) or the high orderliness group processing more item-specific information (i.e., which would also slow down recall early in the first task, due to additional item-specific cues to sample from, however item gain would also be expected later in the recall period). Given that item loss was not related to any of the direct personality measures and that more item gain can be seen from minutes 3 to 10 for the high orderliness group relative to the low and medium groups, the more supported conclusion is that high orderliness individuals processed more itemspecific information than did their counterparts. ${ }^{9}$

\footnotetext{
${ }^{9}$ Group level estimates for the best fitting cumulative recall curve (Equation 1) were calculated for the orderliness groups' self-reference task performance in Study 1 as presented in Figure 3. The low orderliness group's asymptote was $\mathrm{n}(\alpha)=3.99(95 \%$ CI $[3.79,4.19])$ at a rate of $\lambda=.82(95 \%$ CI [.60, $1.05])$; the medium orderliness group's asymptote was $\mathrm{n}(\alpha)=3.90(95 \%$ CI $[3.69,4.11])$ at a rate of $\lambda=.88$ $(95 \% \mathrm{CI}[.61,1.15])$; and the high orderliness group's asymptote was $\mathrm{n}(\alpha)=4.13(95 \% \mathrm{CI}[3.88,4.40])$ at a rate of $\lambda=.49(95 \% \mathrm{CI}[.37, .61])$. These fits suggest that the high orderliness group approached asymptote more slowly than did the medium and low orderliness groups (who did not differ from one another), supporting convergent validity.
} 
Although the exponential function describing cumulative recall (Equation 1) did not adequately fit the recall data from the semantic, rhyming, and structural tasks for individual participants, Figure 3 nevertheless presents a plot of cumulative recall as a function of these three tasks combined. The three curves presented at the bottom of Figure 3 appear as though the rate of approach to asymptote for the combined tasks was approximately equal among those who were low, medium, or high with respect to the direct measure of orderliness. ${ }^{10}$ Although these data must be interpreted with caution, they suggest that making a self-reference judgment was necessary for the negative relationship between the rate of approach and orderliness to hold, thus providing preliminary evidence for divergent validity. ${ }^{11}$

\footnotetext{
${ }^{10}$ Group level estimates for the best fitting cumulative recall curve (Equation 1) were calculated for the orderliness groups' combined task performance in Study 1 as presented in Figure 3. The low orderliness group's asymptote was $n(\alpha)=1.89(95 \%$ CI $[1.70,1.96])$ at a rate of $\lambda=.26(95 \%$ CI $[.22, .31])$; the medium orderliness group's asymptote was $\mathrm{n}(\alpha)=1.57(95 \% \mathrm{CI}[1.37,1.78])$ at a rate of $\lambda=.22(95 \% \mathrm{CI}$ $[.16, .29])$; and the high orderliness group's asymptote was $\mathrm{n}(\alpha)=1.96(95 \% \mathrm{CI}[1.78,2.14])$ at a rate of $\lambda$ $=.36(95 \% \mathrm{CI}[.26, .46])$. These fits suggest that the high orderliness group approached asymptote more quickly, not slowly, than did the medium and low orderliness groups, supporting divergent validity.

${ }^{11}$ In addition, the controlled-IAT data were collected along with the self-report measures of immoderation and neuroticism in an effort to examine divergent validity by examining the interrelationships among those measures and the rate of approach to asymptote for recall of the controlled list encoded in the semantic task. Relationships between the direct and indirect measures and the rate of approach to asymptote were expected to establish divergent validity, in that without a self-reference judgment the rate of approach would not relate to personality measures. Unfortunately, due to very poor recall following semantic encoding, these relationships could not be appropriately examined.
} 


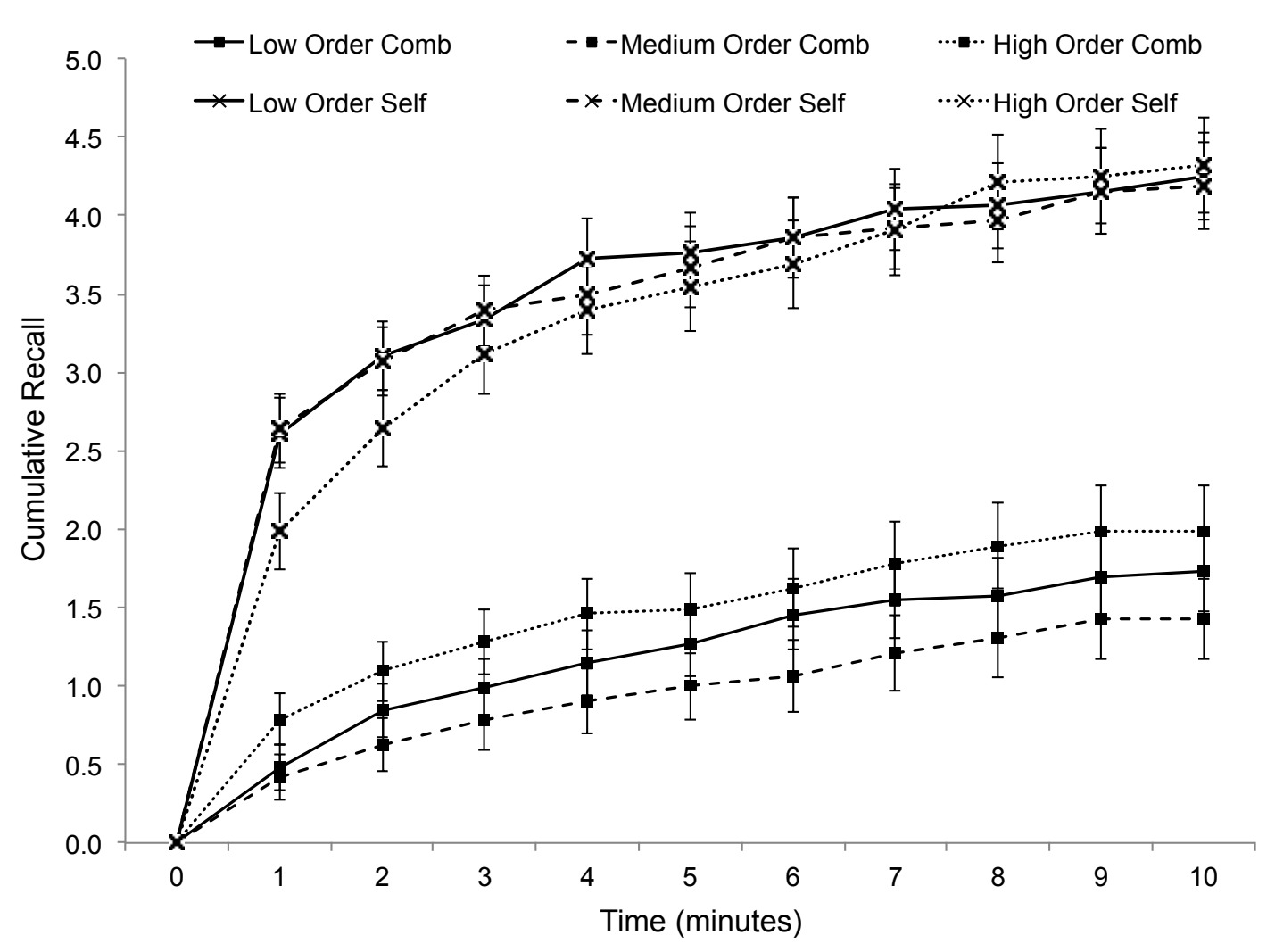

Figure 3. Cumulative recall in Study 1 as a function of orderliness, presented separately for the self-reference and combined semantic, rhyming, and structural tasks. Error bars represent standard error of the mean. Values have been adjusted for reading span differences. Order $=$ Orderliness. Comb $=$ Combined. Self $=$ Self-reference.

In sum, much of the data comparing the direct and indirect personality measures to free recall performance following self-reference encoding suggested that individuals who were highly conscientious and orderly encoded more item-specific information when they made self-reference decisions in the levels-of-processing task, which slowed down recall. Although the largest differences in cumulative recall were early in the first recall task (i.e., minutes 1 and 2), which suggested that the recall speed difference may have been due to relational information, further results showed that conscientiousness and orderliness were not associated with item loss, and they appeared to make larger item 
gains later in the recall period (i.e., minutes 3 through 10). Hence, the most compelling explanation for the slower recall is that participants who were highly orderly and conscientious tapped an explicit personality self-concept that activated more itemspecific information when they encoded the orderliness adjectives, resulting in a larger set of cues to search during retrieval (see Wixted \& Rohrer, 1994).

Self-reported and observed behaviour. Correlations among the measures of conscientious behaviour and personality were examined to address the hypotheses concerning the predictive validity of the memory-based measures of personality selfconcept. Before computing the correlations, the measures of conscientious behaviour were examined for normality and outliers using the criteria adopted in the previous section. The arrival time, lateness, and high school GPA distributions were approximately normal. Histograms of the measures of error made in the $\mathrm{d} 2$ test showed four outlying cases $(z>4.59)$. Once these cases were removed, one of the distributions (omission errors) was log transformed and one was inverse transformed (commission errors). In addition, the distribution of self-reported frequency of window cleaning was log transformed. All distributions were approximately normal after the transformations and after outlying cases were filtered. In the following analyses, outliers and missing data identified in the previous section (with the exception of working memory outliers) were also excluded. Zero-order correlations were computed and only measures of conscientious behaviour that were predicted by personality are presented in Table 5. 
Table 5

Zero-Order Correlations Between Direct and Indirect Personality Measures and Conscientious Behaviour

\begin{tabular}{lcccc}
\hline \multicolumn{1}{c}{ Personality Measure } & $\begin{array}{c}\text { Omission } \\
\text { Error }\end{array}$ & Lateness & $\begin{array}{c}\text { Window } \\
\text { Cleaning }\end{array}$ & $\begin{array}{c}\text { High School } \\
\text { GPA }\end{array}$ \\
\hline 1. Conscientiousness & .10 & $-.23^{*}$ & $.18^{+}$ & $.18^{+}$ \\
2. Orderliness & $.19^{+}$ & $-.14^{++}$ & $.36^{* * *}$ & .11 \\
3. Encoding-Orderliness & $.15^{++}$ & -.05 & $.14^{++}$ & .04 \\
4. Orderliness Recall & -.01 & .00 & .11 & $.19^{+}$ \\
5. Rate of Approach & $.19^{+}$ & .04 & $-.21^{*}$ & -.01 \\
6. Correct Recognition & $-.14^{++}$ & $-.14^{++}$ & .04 & .10 \\
7. False Recognition & $-.24^{*}$ & $.21^{*}$ & -.02 & $-.19^{+}$ \\
8. Orderliness-IAT & $.16^{++}$ & $-.17^{+}$ & -.04 & .06 \\
9. Inverse Item gain ${ }^{\text {a }}$ & $.29^{* *}$ & $.15^{++}$ & -.06 & .04 \\
10. Inverse Item loss ${ }^{\text {a }}$ & -.07 & -.04 & .04 & .10 \\
\hline
\end{tabular}

Note. $n=93-97$.

${ }^{a}$ Inverse transformed. Must be interpreted accordingly.

${ }^{++} p<.20 .{ }^{+} p<.10 .{ }^{*} p<.05 .{ }^{* *} p<.01 .{ }^{* * *} p<.001$.

The week within the semester was not significantly correlated with any of the personality measures $(|r s|<.07)$ nor was the participants' arrival time $(|r s|<.16$ with encoding-orderliness at $r=.15, p=.15$ ) or the number of commission errors made in the $\mathrm{d} 2$ test $(|r \mathrm{~s}|<.04)$. However, as can be seen in Table 5, the number of omission errors in the $\mathrm{d} 2$ test was marginally correlated with self-reported orderliness and the rate of approach parameter. That is, the higher participants' orderliness on a direct measure, the more omission errors that were committed in the $\mathrm{d} 2$ test (i.e., failing to circle a " $\mathrm{d}$ " with 
exactly two primes around it) which is in the opposite direction than was expected. ${ }^{12}$ The significant negative relationship between false recognition and omission error also suggests higher orderliness rendered more omission errors, with higher false recognition hypothesized to be indicative of lower orderliness (i.e., less item-specific information concerning orderliness available in the self-concept). In contrast, the number of omission errors was marginally positively related to the rate of approach parameter in the correct direction. That is, faster recall of the orderliness adjectives was related to more omission errors in the $\mathrm{d} 2$ test, with faster recall hypothesized to be an indicator of lower orderliness. Furthermore, higher correct recognition of the orderliness adjectives tended to be related to fewer omission errors. Hence, two of the measures are counter to expectation, whereas the rate of approach parameter and correct recognition correlated with omission errors in the predicted direction, providing some degree of predictive validity.

Further results showed that self-reported conscientiousness was negatively correlated with lateness, whereas false recognition was positively correlated with lateness. Hence, as was expected, both measures suggested that higher conscientiousness (higher self-reports and lower false recognition) was predictive of earlier arrival times (relative to scheduled times). ${ }^{13}$ Self-reported frequency of window cleaning was significantly correlated in the predicted direction with orderliness and the rate of

\footnotetext{
${ }^{12}$ The encoding-orderliness and orderliness-IAT were approaching marginal significance, also in the opposite direction than what was expected. In particular, there was a tendency for a greater number of omission errors to be related to higher orderliness based on the number of endorsements of orderliness trait adjectives in the self-reference task. Also, there was a tendency for a greater number of omission errors to be related to stronger associations between the self-concept and orderliness as measured by the orderlinessIAT.

${ }^{13}$ Additionally, the direct and indirect measures of orderliness and two memory-based measures were associated with lateness in the predicted direction, but did not reach a conventional level of statistical significance. Specifically, higher orderliness, orderliness-IAT, correct recognition, and item gain was associated with earlier arrival times.
} 
approach parameter (i.e., higher orderliness and lower rate of approach correlated with higher frequency of window cleaning). ${ }^{14}$ Finally, self-reported high school GPA was marginally positively correlated with conscientiousness and orderliness recall, and marginally negatively correlated with the false recognition measure, all in the predicted direction.

The lack of predictive validity for the orderliness-IAT across the measures of conscientious behaviour is particularly noteworthy. It may have been due to a programming issue, wherein blank screens between trials would inexplicably appear occasionally, such that participants were required to press a response key to continue (see Footnote 2). Although participants reported the issue relatively infrequently, the fact that cases were treated unequally in this respect and that response times for trials proceeding a blank screen could be more variable than when trials were unaffected, the IAT results should be interpreted with caution. That is, the marginal predictive validity presented in Table 5 and correlations with direct measures (and the memory-bases ones) presented in Table 4 may have been significant without the additional error variance from the programming issue.

Lastly, it is also noteworthy that the recognition memory test for the levels-ofprocessing stimuli was administered after the two recall trials. Although previous researchers have accepted this methodological issue (e.g., Einstein \& Hunt, 1980), it should be acknowledged that the measures of correct recognition and false recognition in the present research were likely affected by having attempted to retrieve list items in the two free recall tasks participants underwent prior to making recognition judgments.

\footnotetext{
${ }^{14}$ Additionally, the direct measure of conscientiousness and encoding orderliness were associated with higher self-reported window cleaning in the predicted direction, but did not reach statistical significance.
} 
Indeed, research on the testing effect has shown that participants given a free recall test (as opposed to encoding the material a second time) impacted subsequent recognition memory performance by improving recollection while leaving familiarity of the material unaffected (Chan \& McDermott, 2007). This methodological issue may have obscured the correct and false recognition measures, affecting their convergent and predictive validity.

\section{Discussion}

Study 1 found a self-reference effect on free recall and recognition memory performance. The item gain and item loss measures from the second recall trial suggested that the self-reference effect was due to increased levels of elaborative and organizational processing. The partial correlations among the direct and indirect measures of orderliness and measures of elaborative processing supported the idea that self-reference encoding of trait adjectives taps explicit personality self-concept. In particular, the results showed that highly conscientious and orderly participants engaged in more elaborative processing when making self-reference decisions of orderliness adjectives relative to those who were lower on that trait. The more elaborate processing was evidenced by slower recall and marginally less false recognition, suggesting that highly orderly participants encoded more item-specific information. The item-specific information arguably came from a more embellished explicit personality self-concept with respect to orderliness. The direct and indirect measures of orderliness were not associated with item loss, implying that highly orderly participants' self-concept with respect to orderliness did not provide additional relational information, and that the negative personality-recall speed relationship was not due to individual differences in relational processing. 


\section{Study 2}

A possible alternative explanation for the Study 1 results was that the negative personality-recall speed relationships were due to how conscientious and orderly people approach a task more generally, rather than being due to more elaborate processing. That is, highly orderly and conscientious participants may have approached recall more carefully, or slowly. To rule out that possibility and to extend the results to a facet of another Big Five trait, the self-reference task in Study 2 required participants to encode adjectives that were strongly associated with imaginativeness (a facet of openness to experience). Given that the direct measure of openness was unrelated to the direct and indirect personality measures, and self-reference recall speed reported in Study 1, it was a good candidate to use in Study 2 (see Appendix K for the zero-order correlations). I expected that the direct and indirect measures of openness and imaginativeness would be negatively correlated with the rate of approach parameter for the self-referenced imaginativeness adjectives and to false recognition of imaginative lures, and that those in turn would predict attitudes and behaviours associated with openness and imaginativeness (i.e., replicating Study 1 with a different trait). Furthermore, in a test of divergent validity, I expected that self-reported orderliness would not be associated with the rate of approach parameter for the self-referenced adjectives and to false recognition of the lures (i.e., casting doubt on the alternative explanation reported above for Study 1).

Study 2 also reduced the demand characteristics of Study 1 by taking the selfreported personality measurements in the Fall 2012 mass-testing questionnaire, instead of in the lab at the end of the experimental procedure. Unlike the orderly and controlled adjective lists in Study 1, the orderliness and imaginative lists in Study 2 were 
categorically distinct. The separation of the lists in semantic space was incorporated to invoke a relational cue and improve recall following the semantic and shallow encoding tasks in an effort to adequately fit cumulative recall curves (Equation 1) to individual participant data. That is, by separating the list in semantic space, participants may notice the categorical structure of the list and use that knowledge as part of a retrieval plan to narrow the search of memory (i.e., the memory list was more like a related list with two categories than an unrelated list comprised of unique items from disparate categories). This change to the stimulus materials was expected to speed up recall. Furthermore, given that there were new adjectives assigned to the semantic task (i.e., the orderliness adjectives instead of the controlled ones), new synonym judgments were created that appeared to be less ambiguous to the experimenter and research assistant. The unsure response option was removed. Lastly, the programming issue with the IAT was addressed.

\section{Method}

Participants. Ninety-two undergraduate psychology students who were not tested in Study 1 were recruited from SONA. Sixty-four participants were female (69.6\%). Only students who completed mass-testing at the beginning of the Fall academic term were eligible to participate.

\section{Materials.}

Levels-of-processing. The orderly and disorderly lists from Study 1 were combined with two new lists of 10 adjectives representing imaginativeness and unimaginativeness. The orderliness and imaginativeness lists were maximally separated in latent semantic space (i.e., distinct orderliness and imaginativeness categories) to 
encourage more organizational processing and use of relational information during recall. In doing so, some of the orderliness adjectives used in Study 1 were exchanged for new ones due to their relatively high association to the imaginativeness concept. Specifically, the orderly adjective "meticulous" was exchanged for "careful", and the disorderly adjectives "chaotic" and "contradictory" were exchanged for "rash" and "impulsive". Table 6 presents the memory lists. Appendix L presents the steps taken in creating the memory lists.

Table 6

Levels-of-Processing Items for the Imaginativeness and Orderliness Facets Memory Lists

\begin{tabular}{cccc} 
Imaginative & Orderly & Unimaginative & Disorderly \\
\hline Curious & Punctual & Passionless & Disorderly \\
Eccentric & Organized & Realistic & Negligent \\
Visionary & Efficient & Superficial & Careless \\
Reflective & Particular & Uninspired & Lax \\
Artistic & Careful & Indifferent & Confused \\
Inventive & Strict & Conventional & Rash \\
Poetic & Precise & Unromantic & Sloppy \\
Imaginative & Accurate & Mundane & Forgetful \\
Creative & Measured & Commonplace & Impulsive \\
Deep & Prompt & Traditional & Unpredictable \\
\hline
\end{tabular}

Note. Items in bold have been used in previous research on conscientiousness- or openness-IATs.

The imaginative and orderly lists were equated in terms of word frequency, word length, likeableness, and meaningfulness. In addition, these lists differed markedly with respect to their LSA to the imaginativeness and orderliness concepts. Specifically, the 
imaginative list had a significantly higher LSA to the imaginativeness concept than did the orderly list. Likewise, the orderly list had a significantly higher LSA to the orderliness concept than did the imaginative list. Hence, the lists that were encoded in the self-reference and semantic tasks were categorically distinct (unlike the orderly and controlled lists in Study 1; see Table 2), which was hypothesized to encourage organizational processing making salient relational information to aid recall. Table 7 presents the psycholinguistic properties of the memory lists.

Table 7

Mean Psycholinguistic Properties for the Imaginativeness and Orderliness Memory Lists

\begin{tabular}{cccccc}
\hline & Imaginative & Orderly & Unimaginative & Disorderly & $F$ \\
\hline Word Frequency & $2.31(.67)$ & $2.53(.60)$ & $1.76(.60)$ & $2.20(.49)$ & $2.97^{*}$ \\
Word Length & $8.10(2.02)$ & $7.80(1.32)$ & $10.30(1.42)$ & $7.90(2.92)$ & $3.46^{*}$ \\
Likeableness $^{\mathrm{a}}$ & $416.00^{\mathrm{b}}$ & $419.00^{\mathrm{c}}$ & $246.00^{\mathrm{d}}$ & $205.57^{\mathrm{e}}$ & \\
& $(83.73)$ & $(76.30)$ & $(124.96)$ & $(70.28)$ & $10.48^{* * *}$ \\
Meaningfulness $^{\mathrm{a}}$ & $357.00^{\mathrm{b}}$ & $363.14^{\mathrm{c}}$ & $344.00^{\mathrm{d}}$ & $370.86^{\mathrm{e}}$ & \\
& $(13.25)$ & $(17.08)$ & $(21.73)$ & $(15.00)$ & 2.66 \\
Imaginative LSA & $.27(.13)$ & $0.12(.07)$ & $.19(.09)$ & $0.09(.06)$ & $8.31^{* * *}$ \\
Orderliness LSA & $.10(.03)$ & $0.21(.09)$ & $.10(.05)$ & $0.10(.06)$ & $6.76^{* * *}$ \\
\hline
\end{tabular}

Note. Standard deviation is presented within parentheses. LSA = latent semantic association.

${ }^{a}$ Calculated from Anderson's (1968) personality trait norms. ${ }^{b}$ missing data for poetic, deep, reflective, and visionary. ${ }^{\mathrm{c}}$ missing data for organized, particular, and measured. ${ }^{\mathrm{d}}$ missing data for traditional, mundane, uninspired, passionless, commonplace ${ }^{\mathrm{e}}$ missing data for disorderly, lax, confused, and rash.

${ }^{*} p<.05 .^{* *} p<.01{ }^{* * *} p<.001$. 
The orienting question for the structural encoding task was changed to "One or more s?", such that the correct response for one-half of the questions was "Yes". The five orienting questions that required a "Yes" within the rhyming and semantic tasks were changed such that the word replacing the "XXXX" was indeed a close rhyme or synonym of the particular trait adjective. The words that replaced the "XXXX" leading to a "No" response were the same as those used in Study 1. Lastly, "Unsure" was not offered as a response option.

Working memory. The same as Study 1.

Recognition memory. In addition to the 40 old orderliness and imaginativeness adjectives, 40 lures and 20 unrelated adjectives were carefully selected. The 40 lures were selected in the same way as the 40 old items, in that 20 adjectives were selected to maximally represent the imaginativeness concept and 20 were selected to maximally represent the orderliness concept. As in Study 1, the 20 unrelated lures were selected from the pool of adjectives describing physical dimensions of height, weight, and strength. The ones that carried the lowest LSA to the trait adjective concepts were selected. Appendix L presents the lures and descriptive and inferential statistics for word frequency, word length, meaningfulness, likeableness, LSA to the orderliness concept, and LSA to the imaginativeness concept.

Implicit Association Test. An imaginativeness-IAT was created from the imaginative and unimaginative lists, along with the "Me" and "Not Me" concepts from Study 1.

Imaginativeness behaviour. Zhiyan and Singer's (1996) research on daydreaming and personality has shown that positive constructive daydreaming is highly related to 
openness to experience $(r=.52)$. Positive constructive daydreaming has been characterized by a positive attitude toward daydreaming and a view that daydreaming is useful for problem solving. In an effort to obtain an in-lab measure of open and imaginative behaviour, a daydreaming task was created. Participants were asked to solve a "lumberjack puzzle" composed of six identical wooden pieces each with one jagged side. The puzzle could be configured into one solid mass. Participants were shown the puzzle pieces and a picture of the solution. Before solving the puzzle, they listened to a passage played on a compact disk player while looking outside at the trees. They were told that the passage was designed to set the stage for a potential daydream that could help them solve the puzzle. It combined general aspects of the visual scene outside with the puzzle pieces. Participants were told that they are free to continue in the daydream once the passage ended. The amount of time participants spent in the daydream after the passage ended was measured. They were then presented the puzzle pieces and solution. After two minutes, the researcher explained that the quality of the daydream that may have been explored was of particular interest, not their ability to solve to puzzle. Participants were asked to rate the vividness of the daydream on a scale from 1(vague impressions) to 7 (vivid and detailed). I hypothesized that participants who were highly open to experience and imaginative would engage in the daydream for more time and they would report a more vivid daydream than those who were less open and imaginative.

On an exploratory basis, participants were asked if they wondered or were curious about anything while they were engaged in the experiment. The researcher recorded the number of curiosities and the content. Lastly, the researcher recorded whether 
participants decided to take the debriefing form as a final behavioural measure of openness.

Attitude and behaviour self-report. The questionnaire used in Study 1 was supplemented with some additional questions that have been shown to relate to openness to experience. Research has shown a very high correspondence between self-reported openness and how open one's ideal romantic partner would be $(r=.54$ and $r=.81$; Figueredo, Sefcek, \& Jones, 2006). One item therefore asked to what extent participants' ideal romantic partner would be open to new experiences. In addition, they were asked to report the likelihood (as a percentage) that they would vote for the conservative political party if an election were called today.

The questions Paunonen (2003) identified as being related to openness in multiple studies were included. In particular, one item asked if participants play a musical instrument and one measured their self-perceived intelligence relative to their peers. In addition, one item asked whether they were pursuing a degree in liberal or non-liberal arts. Finally, participants were asked if they enjoy reading classic literature. Schmukle, Back, and Egloff (2008) found that an openness-IAT $(r=.25)$ and self-reported openness $(r=.34)$ predicted whether participants read classic literature. Moreover, the partial correlation between classic literature and the openness-IAT was significant, after controlling for self-reported openness and gender $(r=.21)$.

Personality self-report. The 40 IPIP items measuring conscientiousness $(\alpha=.79)$, orderliness $(\alpha=.84)$, openness $(\alpha=.79)$, and extraversion $(\alpha=.87)$ from Study 1 were included in the Fall 2012 mass-testing questionnaire. In addition, 9 IPIP items measuring imaginativeness ( 1 item overlapped the openness scale and therefore was only included 
once; $\alpha=.80)$ and 10 filler items measuring sociability $(\alpha=.85)$ were included. In total, the questionnaire consisted of 59 items.

Design. The same as Study 1.

Procedure. For the levels-of-processing task, the disorderly, unimaginative, orderly, and imaginative lists were assigned to the structural, rhyming, semantic, and selfreference encoding tasks respectively. The order of presentation of the encoding tasks, orienting questions, and adjectives was kept consistent with Study 1 where possible. The presentation order for the adjectives in the disorderly list presented in the structural task was the same as in Study 1, except the two new adjectives replaced the two that were removed. The order of presentation of the adjectives in the unimaginative list in the rhyming task was quasi-randomized, such that one-half were assigned a "No" response and one-half a "Yes" response, keeping the order of responses the same as in Study 1. Furthermore, the orienting questions within the rhyming task were presented in the exact same order as in Study 1, except the new word replacing the "XXXX" in the orienting questions that required a "Yes" response was specific to the adjective assigned to that trial (e.g., "Realistic" was assigned to the first trial of the rhyming task and it required a "Yes" response, so a new rhyme word "Ballistic" replaced the "XXXX" in the orienting question). A similar procedure was applied to the adjectives in the orderly list presented in the semantic task, except that the order of presentation was not quasi-randomized to keep the order the same as in Study 1. The order of presentation of the adjectives in the imaginative list presented in the self-reference task was randomized. Finally, the primacy and recency trials were identical to those from Study 1, except the first trial required a 
"No" response for the word "Green" because the structural orienting task was changed from "Two or more e's?" to "One or more s?".

For the recognition task, one randomized order of the items was presented. Following recognition, the imaginativeness-IAT was conducted using the seven-block structure from Study 1 (i.e., Blocks 1 through 7 in Table 3). Additionally, to address the programming issue identified in Study 1, the IAT was reprogrammed in an updated version of SuperLab (version 4.5) and administered on a newer iMac computer. After the IAT, participants completed the behaviour self-report questionnaire in the conference room. The daydreaming task was then administered. Lastly, the curiosity measure was taken, and participants were presented the debriefing form. They specifically were told that the form explains the nature of the research and that they are welcome to take it or leave it upon departure. All other aspects of the procedure were the same as in Study 1.

\section{Results}

Hypotheses were tested in the same manner as in Study 1 with the following exception: zero-order correlations were computed instead of partial correlations because the reading span measure of working memory capacity did not correlate with the variables of interest in Study 1. In addition, to establish divergent validity and assess the alternative explanation for the main results of Study 1, zero-order correlations between orderliness and conscientiousness and the direct, indirect, and memory-based measures of openness and imaginativeness were examined.

Self-reference effect. Two participants were excluded from analyses due to chance accuracy (i.e., .50 correct) on at least two of the three encoding tasks where accuracy was applicable (i.e., structural, rhyming, and semantic). 
The main effect of encoding task on RTs for the judgments made in the levels-ofprocessing task was significant, $F(1.91,170.08)=10.53, \eta^{2}=.11, p<.001$. The structural $(M=1,187 \mathrm{~ms}, S E=71 \mathrm{~ms})$ and rhyming $(M=1,134 \mathrm{~ms}, S E=42 \mathrm{~ms})$ tasks were executed faster than the semantic $(M=1,313 \mathrm{~ms} S E=47 \mathrm{~ms})$ task, $M_{\text {diff }}=-126 \mathrm{~ms}$, $S E=59 \mathrm{~ms}, p=.04$ and $M_{\text {diff }}=-178 \mathrm{~ms}, S E=35 \mathrm{~ms}, p<.001$, respectively. The structural and rhyming tasks were also executed faster than the self-reference task $(M=$ $1,378 \mathrm{~ms} S E=47 \mathrm{~ms}), M_{\mathrm{diff}}=-190 \mathrm{~ms}, S E=65 \mathrm{~ms}, p<.01$ and $M_{\mathrm{diff}}=-243 \mathrm{~ms}, S E=41$ ms, $p<.001$, respectively. Lastly, the semantic task was executed faster than the selfreference task, $M_{\text {diff }}=-65 \mathrm{~ms}, S E=30 \mathrm{~ms}, p=.04$, and the difference in RT between the structural and rhyming tasks was not significant, $p>.05$. Of note is that RT in the semantic task was $241 \mathrm{~ms}$ faster than in Study 1, suggesting the synonym judgments were less ambiguous.

For the ANOVA on accuracy, the main effect of encoding task was significant, $F(1.51,134.09)=50.40, \eta^{2}=.36, \mathrm{p}<.001$. The responses provided in the structural $(M$ $=.83, S E=.02)$ task were less accurate than those provided in the rhyming $(M=.97, S E$ $=.007)$ and semantic tasks $(M=.93 S E=.007), M_{\text {diff }}=-.14, S E=.02, p<.001$ and $M_{\text {diff }}$ $=.10, S E=.02, p<.001$, respectively. The responses made in the rhyming task were more accurate than the responses made in the semantic task, $M_{\mathrm{diff}}=.04, S E=.01, p<$ .001. Importantly, accuracy in the semantic task improved relative to Study 1 (i.e., $M=$ .93 vs. $M=.77$ ), providing further evidence that the synonym judgments were less ambiguous.

The two-way repeated-measures ANOVA on proportion recalled revealed a significant main effect of encoding task, $F(2.52,224.27)=267.08, \eta^{2}=.75, p<.001$, 
representing a strong levels-of-processing effect. Specifically, the self-reference task yielded higher recall $(M=.40, S E=.015)$ than did the semantic $(M=.15, S E=.012)$, rhyming $(M=.04, S E=.007)$, and structural $(M=.06, S E=.008)$ tasks, $p$ s $<.001$. In turn, the semantic task yielded higher recall than did the rhyming and structural tasks, $p \mathrm{~s}$ $<.001$. The structural task yielded higher recall than did the rhyming task, $p=.047$. The main effect of recall trial was marginally significant, $F(1,96)=3.63, \eta^{2}=.036, p=.057$, indicating that participants tended to recall more items on the second recall trial than the first trial (i.e., hyperamnesia; e.g., Klein et al., 1989). Finally, the Encoding Task x Recall Trial interaction was not significant, $F(2.15,191.13)=.34, \eta^{2}=.004, p=.73$. Other than replicating the levels-of-processing effect, these results reveal that recall in the semantic task doubled from $M=.07$ in Study 1 to $M=.15$ in Study 2. The increase in recall may have been due to removal of the unsure response option, implementing clearer synonym judgments, or separation of the word lists in semantic space, which was predicted to elicit organizational processing to aid recall. The overall amount recalled in the semantic and shallow encoding tasks, however, was still too low to fit cumulative recall curves to those data. Figure 4 presents cumulative recall as a function of time for each encoding task. 


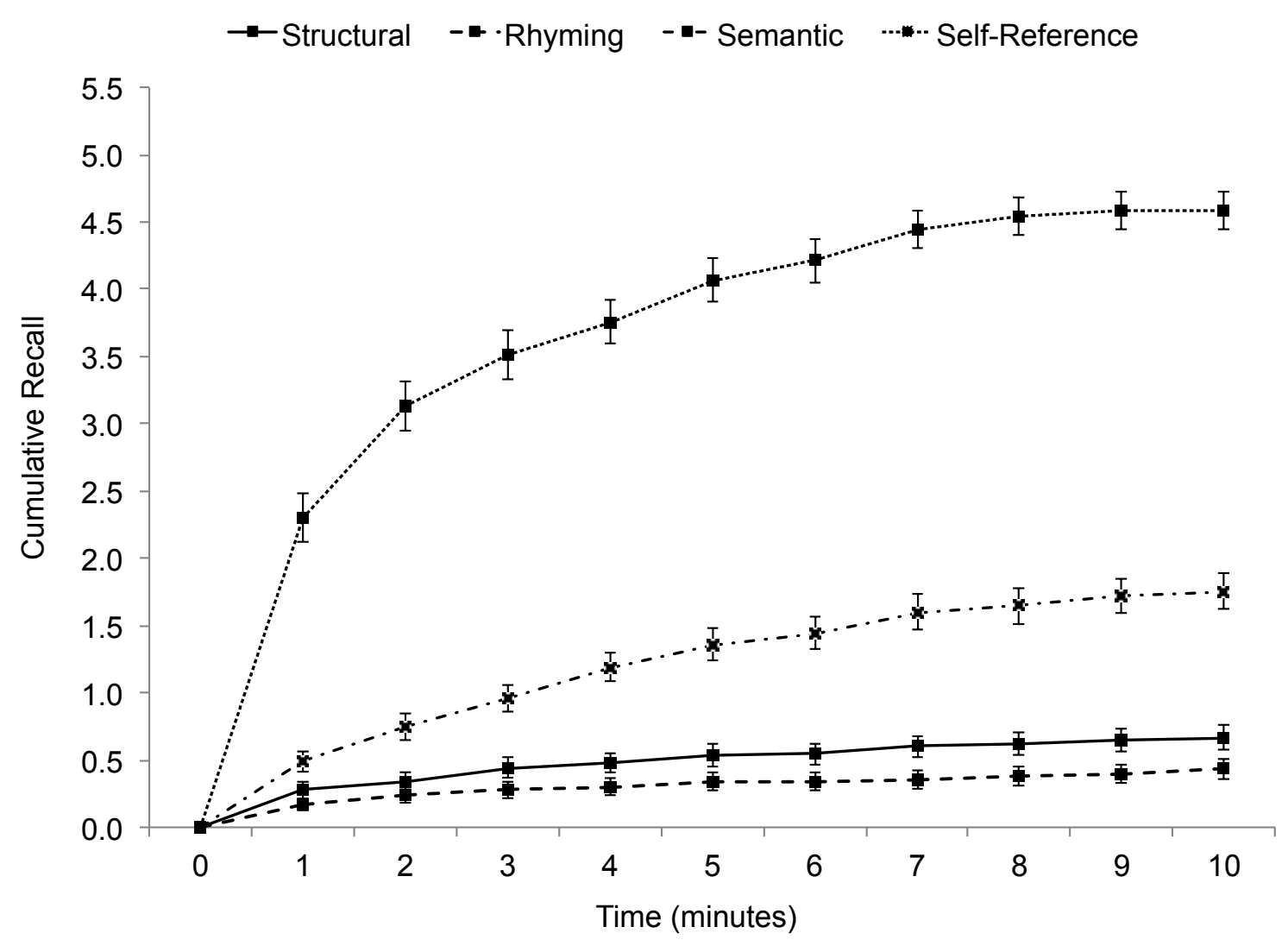

Figure 4. Cumulative recall in Study 2 as a function of encoding task. Error bars represent standard error of the mean.

Analysis of item gain revealed a significant main effect of encoding task, $F(2.30$, $205.01)=8.04, \eta^{2}=.08, p<.001$. Item gain was higher following self-reference $(M=$ $.046, S E=.009)$ and semantic $(M=.044, S E=.005)$ encoding than it was following rhyming $(M=.009, S E=.003)$ and structural $(M=.016, S E=.004)$ encoding, $p \mathrm{~s}<.01$. Item gain did not differ between the structural and rhyming tasks, $p=.22$. Unlike Study 1, the self-reference task did not elicit higher item gain than did the semantic task, $p=$ .93 , suggesting similar amounts of item-specific information were driving recall. ${ }^{15}$

\footnotetext{
${ }^{15}$ However, when openness (low, medium, high) was added as a between-subjects variable in the ANOVA, the Encoding Task $x$ Openness interaction was marginally significant, $F(4.70,204.22)=1.97, \eta^{2}=.04, p=$ .09. Customized interaction contrasts showed that participants who were highly open to experience gained more self-referenced adjectives in the second recall trial than semantic adjectives relative to those who reported medium openness $\left(M_{\text {contrast }}=.074, S E=.03, p=.01\right)$. The contrast comparing the high and low
} 
Analysis of item loss revealed a statistically significant main effect of encoding task, $F(2.25,196.09)=4.30, \eta_{\mathrm{p}}^{2}=.05, p=.01$. Follow-up comparisons showed that the proportion of item loss was lower following rhyming $(M=.03, S E=.02)$ encoding than it was following semantic $(M=.16, S E=.03)$ and self-reference $(M=.09, S E=.02)$ encoding, $M_{\mathrm{diff}}=.13, S E=.04, p=.003$ and $M_{\mathrm{diff}}=.06, S E=.02, p=.02$, respectively. The difference in item loss between the structural $(M=.07, S E=.03)$ and semantic tasks was marginally significant, $M_{\text {diff }}=.09, S E=.05, p=.06$. Most importantly, although the semantic task elicited more item loss than did the self-reference task, the difference was only marginally significant, $M_{\mathrm{diff}}=.07, S E=.04, p=.07$. However, it should be noted that the rate of item loss in the structural, rhyming, and semantic tasks was artificially reduced by the number of participants who did not recall any of the adjectives presented in theses tasks (making item loss impossible). Specifically, there were 52 participants (57.8\%) who did not recall any structurally encoded adjectives by the end of the first recall task, 64 participants $(71.1 \%)$ who did not recall any for the rhyming task, and 22 participants $(24.4 \%)$ who did not recall any for the semantic task, whereas there were only 3 participants $(0.3 \%)$ who did not recall any for the self-reference task. The rate of item loss after excluding theses participants increased for the structural $(M=.17)$, rhyming $(M=.12)$, and semantic $(M=.21)$ tasks, but not for the self-reference task $(M=$ .09), which had the nominally lowest rate of item loss, as was expected (Klein et al., 1994). An additional test among the 64 participants who recalled adjectives encoded in the semantic and self-reference tasks by the end of the first recall trial revealed

openness groups, however, did not reach statistical significance $\left(M_{\text {contrast }}=.037, S E=.030, p=.22\right)$, $F(2,87)=3.15, \eta_{\mathrm{p}}^{2}=.07, p=.05$. Hence, the self-reference task elicited higher item gain (relative to the semantic task) among participants who were highly open to experience (relative to those who were moderately open to experience), suggesting that the extent to which elaborative processing drove the selfreference effect was moderated by the degree to which the stimulus materials were self-relevant. 
significantly higher item loss in the semantic task $(M=.20, S E=.05)$ than the selfreference task $(M=.09, S E=.02), F(1,62)=3.62, \eta^{2}=.08, p=.02$. Hence, these results suggest that self-reference encoding elicited more relational information than did semantic encoding, confirming that the self-reference effect was in part due to organizational processing. ${ }^{16}$

The ANOVA on correct recognition revealed a significant main effect of encoding task, $F(2.48,220.48)=228.09, \eta^{2}=.72, p<.001$. Specifically, trait adjectives subjected to self-reference encoding $(M=.96, S E=.008)$ were better recognized as having been presented in the levels-of-processing task than were those encoded in the semantic $(M=.84, S E=.015)$, rhyming $(M=.53, S E=.020)$, and structural $(M=.47, S E$ $=.023)$ tasks, $p \mathrm{~s}<.001$. In turn, the trait adjectives presented in the semantic task were better recognized than those presented in the rhyming and structural tasks, $p \mathrm{~s}<.001$. Lastly, the trait adjectives presented in the rhyming task were better recognized than those presented in the structural task, $p<.01$, although both were close to chance accuracy (i.e., $M=.50$ ). This pattern of data mirrors the free recall results, with the exception that the structural task produced slightly higher recall but slightly worse correct recognition than did the rhyming task.

Another ANOVA on proportion of yes responses in the recognition task revealed a significant main of item type, $F(1.99,176.83)=1952.40 \eta^{2}=.96, p<.001$. Participants responded yes to old items $(M=.70, S E=.011)$ more often than to lures $(M$ $=.20, S E=.012)$ and unrelated items $(M=.03, S E=.005), p s<.001$. In turn, participants

\footnotetext{
${ }^{16}$ Note that the rate of item loss for the semantic and self-reference tasks decreased relative to Study $1(M=$ .31 vs. $M=.20$ for the semantic task and $M=.15$ vs. $M=.09$ for the self-reference task). These results suggest that there was indeed more organizational processing of the adjectives, as was expected after separating the memory lists into distinct categories (i.e., a related list instead of an unrelated one).
} 
responded yes to lures more often than to unrelated items, $p<.001$. The difference between the unrelated items and the lures suggests significant false recognition of the adjectives that were not presented in the levels-of-processing task.

Lastly, an interaction contrast tested the difference in correct and false recognition in the self-reference task to the difference in correct and false recognition in the semantic task. The interaction contrast was statistically significant, $M_{\text {difference }}=-.11, S E=0.02, F(1$, $89)=20.90, \eta^{2}=.19, p<.001$. Follow-up comparisons revealed that the proportion of false recognition did not differ between the self-reference $(M=.24, S E=.02)$ and semantic $(M=.23, S E=.02)$ tasks, $M_{\text {difference }}=-.01, S E=0.02, p=.61$, whereas correct recognition differed significantly between the self-reference $(M=.96, S E=.01)$ and semantic $(M=.84, S E=.02)$ tasks, $M_{\text {difference }}=-.12, S E=0.02, p<.001$. Thus, the processing differences involved in the self-reference effect impacted correct recognition but did not impact false recognition. Alternatively, these result may be explained by a testing effect, wherein engaging in retrieval of list items during free recall prior to taking the recognition test enhanced correct recognition but did not impact false recognition (e.g., by increasing recollection of list items but leaving familiarity relatively unaffected; Chan \& McDermott, 2007).

To summarize, the memory results showed that participants completed the levelsof-processing task accurately, including the semantic task. The changes made to the synonym judgments in the semantic task lead to faster RTs, higher accuracy, and recall doubled from $M=.07$ in Study 1 to $M=.15$ in Study 2, which was close to the level of free recall reported in Rogers et al.’s (1977; Experiment 1) synonym judgment task (i.e., $M=.13)$. Furthermore, the results confirmed a strong self-reference effect on recall and 
recognition. Unlike Study 1, item gain did not differ between the semantic and selfreference tasks, suggesting, likely incorrectly, that elaborative processing did not drive the self-reference effect. Analysis of item loss revealed that self-reference encoding yielded more organizational processing than did the semantic task, suggesting the selfreference effect was being driven by relational information. Relative to Study 1 , separating the memory lists in semantic space nominally increased the log transformed rate of approach to asymptote for the self-referenced adjectives (Study 2: $M=.33,95 \%$ CI $[.26, .39]$ vs. Study $1: M=.06,95 \%$ CI $[-.24, .36])$ and decreased item loss in the semantic and self-reference tasks (see Footnote 16), suggesting participants encoded the additional relational information. It is possible that the item gain difference was not significant in Study 2 because the additional item-specific information encoded in the self-reference task elicited successful retrieval of list items earlier in the 10 minutes of recall, due to encoding and use of more effective relational information (Burns \& Hebert, 2005; Burns \& Schoff, 1998).

Personality and self-reference. Normality of the distributions and outliers were examined under the same criteria as Study 1 (Tabachnick \& Fidell, 2007). The distribution for the estimated rate of approach to asymptote was log transformed, while the item gain and item loss distributions were inverse transformed. There was one outlier in the encoding-openness distribution $(z=-3.18)$, two outliers in the false recognition $(z \mathrm{~S}$ $=3.12)$ and inverse transformed item gain distributions $(z \mathrm{~s}=4.58)$, and one outlier in the inverse transformed item loss distribution $(z=-3.70)$. In the following analyses, the transformed variables were examined and outliers were only excluded when the respective variable or combinations of variables were involved in a particular correlation. 
Table 8 displays a zero-order correlation matrix with the direct and indirect personality measures as well as the elaborative and organizational processing measures for self-referenced openness adjectives. As one would expect, the direct measure of openness and imaginativeness derived from self-report ratings of dispositional phrases were significantly positively correlated. Additionally, the self-reference judgments made regarding the openness adjectives in the levels-of-processing task (i.e., encodingopenness) correlated positively with the direct measures of openness and imaginativeness, suggesting a similar source of knowledge was tapped when making personality judgments for all three measures. 
Table 8

Zero-Order Correlations Among Direct and Indirect Personality Measures and Self-Reference Memory Processes in Study 2

\begin{tabular}{|c|c|c|c|c|c|c|c|c|c|c|}
\hline Measure & 1 & 2 & 3 & 4 & 5 & 6 & 7 & 8 & $9^{\mathrm{a}}$ & $10^{\mathrm{a}}$ \\
\hline 1. Openness & - & & & & & & & & & \\
\hline 2. Imaginativeness & $.40^{* * *}$ & - & & & & & & & & \\
\hline 3. Encoding-Openness & $.36^{* * *}$ & $.33^{* *}$ & - & & & & & & & \\
\hline 4. Openness Recall & $.20^{+}$ & $.20^{+}$ & -.01 & - & & & & & & \\
\hline 5. Rate of Approach & $-.24^{*}$ & .04 & -.03 & -.11 & - & & & & & \\
\hline 6. Correct Recognition & .05 & -.02 & -.03 & $.22^{*}$ & $.18^{+}$ & - & & & & \\
\hline 7. False Recognition & .05 & .04 & .11 & $-.14^{++}$ & .03 & .06 & - & & & \\
\hline 8. Openness-IAT & -.01 & -.04 & $-.17^{++}$ & .01 & -.03 & $-.23^{*}$ & .03 & - & & \\
\hline 9. Inverse Item gain ${ }^{a}$ & -.09 & $-.16^{++}$ & .02 & $-.18^{+}$ & $.30^{* *}$ & .09 & $-.17^{++}$ & .01 & - & \\
\hline 10. Inverse Item loss ${ }^{a}$ & -.07 & $-.19^{+}$ & $-.22^{*}$ & $-.19^{+}$ & .08 & .06 & -.02 & .03 & $.16^{++}$ & - \\
\hline
\end{tabular}

Note: $n=86$ to $90 .^{\text {a }}$ Inverse transformed. Must be interpreted accordingly. Two-tailed tests.

${ }^{++} p<.20 .{ }^{+} p<.10 .^{*} p<.05 .{ }^{* *} p<.01 .^{* * *} p<.001$. 
Of particular interest was the relationship between the rate of approach to asymptote and the direct and indirect personality measures. As was hypothesized, openness was negatively related to the rate of approach parameter, extending the findings from Study 1 to a different Big Five personality trait. Unlike Study 1, however, the more specific facet (imaginativeness) and encoding-openness were not associated with the rate of approach parameter. Further results showed that correct recognition was not associated with the direct measures of personality. As one would expect, correct recognition was associated with openness recall. Unlike what was observed in Study 1, there was a marginally positive association between correct recognition and the rate of approach parameter, with higher correct recognition being associated with faster recall. This result suggests that correct recognition of the self-referenced adjectives may have been driven somewhat more by relational cues than by item-specific information. As was discussed in the previous section, separating the semantic and self-reference memory lists into two distinct categories instead of one seemed to speed-up recall and reduce item loss, both indicating the use of more effective relational information. This information appears to have helped participants make correct recognition judgments.

Unlike the consistent trends observed in Study 1, Table 8 shows that false recognition was not associated with the direct measures of openness and imaginativeness. Hence, Study 2 did not support the hypothesis that people who are highly open or imaginative would falsely recognize fewer openness lures in the recognition test, due to activation of more item-specific information in their personality self-concept during selfreference encoding. Despite that, the data did suggest that higher openness recall was marginally associated with less false recognition of openness lures, indicating that the 
processing that lead to higher recall may have also been involved in preventing false recognition (which was hypothesized to be item-specific information. The indirect measure of openness (openness-IAT) was not consistently related to the three direct measures, except that encoding-openness tended to be related to the openness-IAT in the opposite direction than was expected. That is, participants who endorsed more of the openness adjectives in the self-reference task tended to have weaker self-openness associations in the IAT. Moreover, weaker openness-IAT effects were significantly associated with higher correct recognition scores. Of particular interest was that the openness-IAT was not associated with the rate of approach parameter or to openness recall, suggesting that the item-specific and relational processing engaged during selfreference did not tap the relative strength of associations in the implicit personality selfconcept (Schnabel \& Asendorpf, 2010; Gawronski \& Bodenhaussen, 2011).

The final two rows of Table 8 present the inverse item gain and inverse item loss correlations. The direct measure of openness and encoding-openness were not related to item gain. However, the direct measure of imaginativeness was marginally related to item gain and openness recall. More specifically, participants who were highly imaginative recalled marginally more openness adjectives by the end of the second recall task and tended to gain more items in the second recall trial than their counterparts, suggesting that they engaged in more item-specific processing. Interestingly, the direct measure of imaginativeness was also marginally related to item loss, with higher imaginativeness being associated with more item loss, indicating that highly imaginative participants engaged in less organizational processing than did other participants. This result further suggests that the higher recall noted among highly imaginative participants was not due 
to relational processing, but instead was due to item-specific processing, a point I return to toward the end of the next section.

Personality and cumulative recall. To better understand the time course of recall as a function of openness, Figure 5 plots cumulative recall for participants who were low $(n=30)$, medium $(n=33)$, or high $(n=27)$ on the direct measure of openness.

Comparing cumulative recall for self-referenced adjectives between the low and medium openness groups (i.e., the solid and coarsely dashed lines at the top of Figure 5), recall was approximately equivalent at minute 1 , the medium openness group recalled nominally more items than the low openness group at minute 2 , and the recall difference between the groups persisted throughout the recall period. This pattern of cumulative recall was likely due to differences in relational information, given that the recall advantage occurred earlier in the first recall task (i.e., minute 2) and persisted throughout. Unfortunately, the item loss data did not corroborate this explanation. However, if the recall difference was due to item-specific information, one would have expected the item gains to be more gradual over the time course of recall. Furthermore, comparing the cumulative recall of the high openness group to the medium and low openness groups provides more insight concerning the relative contributions of relational and item-specific information. More precisely, the pattern of recall over time for the highly open individuals shows that they recalled nominally fewer adjectives than other participants at minute 1 but more adjectives by minute 10, with their cumulative recall exceeding the low openness group by minute 2 and the medium group by minute 7 . This pattern suggests persistent item gains among the high openness group across the recall period relative to both the low and medium groups. If the high openness group encoded more 
relational information than the other groups, they would have had higher cumulative recall early in the first recall task (e.g., minute 1 or 2), which clearly did not occur. Thus, the pattern of cumulative recall suggests that high levels of openness yielded more itemspecific information when encoding the openness adjectives self-referentially, replicating Study 1 with a different trait. ${ }^{17}$

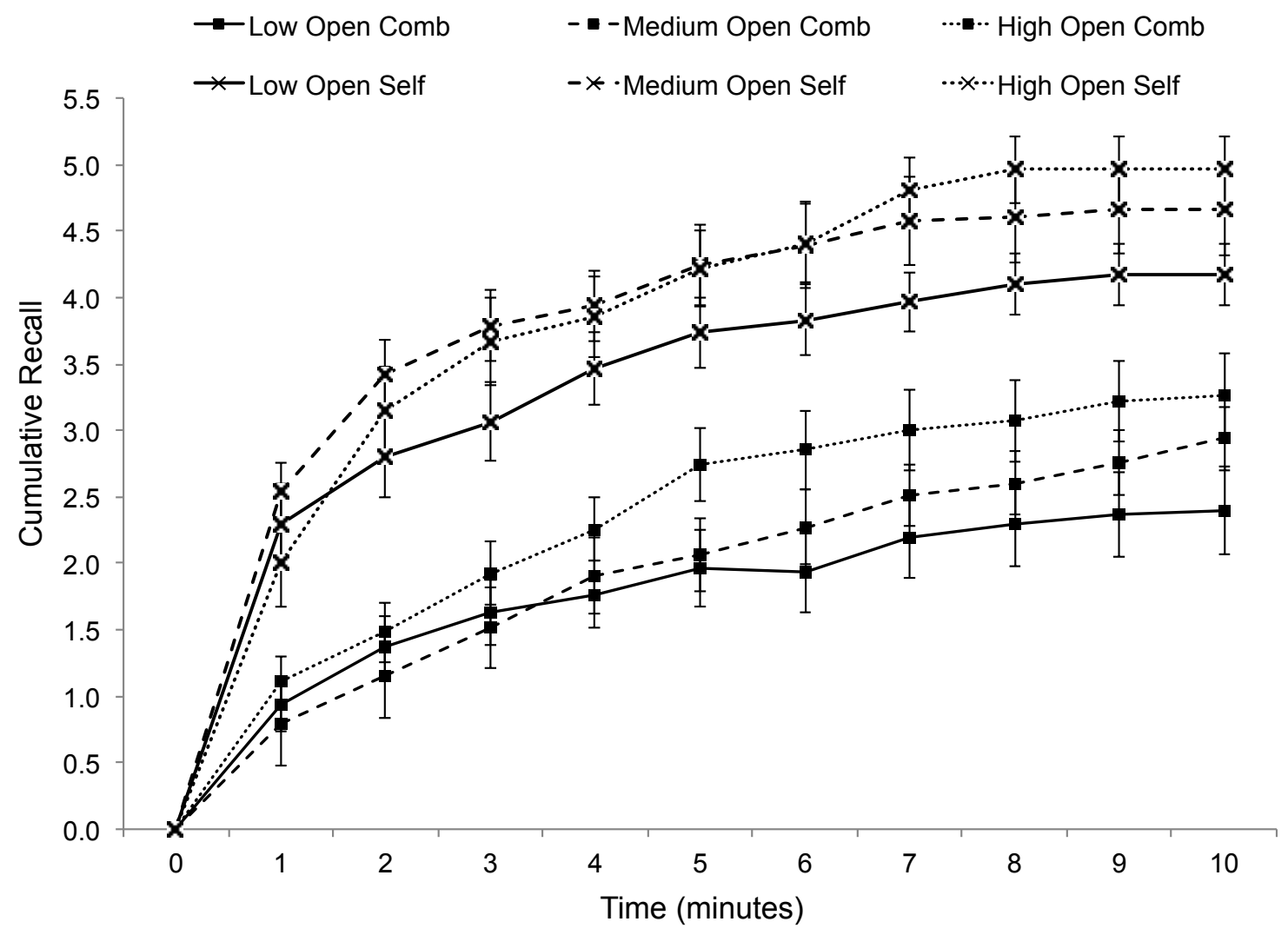

Figure 5. Cumulative recall in Study 2 as a function of openness presented separately for the self-reference and combined semantic, rhyming, and structural tasks. Error bars

\footnotetext{
${ }^{17}$ Group level estimates for the best fitting cumulative recall curve (Equation 1) were calculated for the openness groups' self-reference task performance in Study 2 as presented in Figure 5. The low openness group's asymptote was $\mathrm{n}(\alpha)=4.02,(95 \%$ CI $[3.79,4.26])$ at a rate of $\lambda=.61,(95 \%$ CI $[.45, .76])$; the medium openness group's asymptote was $\mathrm{n}(\alpha)=4.53(95 \%$ CI $[4.35,4.71])$ at a rate of $\lambda=.69(95 \% \mathrm{CI}$ $[.56, .82])$; and the high openness group's asymptote was $\mathrm{n}(\alpha)=4.94(95 \% \mathrm{CI}[4.70,5.18])$ at a rate of $\lambda=$ $.45(95 \%$ CI $[.37, .53])$. These fits confirm that the high openness group approached asymptote more slowly than did the low and medium openness groups (who did not differ from one another).
} 
represent standard error of the mean. Open $=$ Openness. Comb $=$ Combined. Self $=$ Selfreference.

Another note about Figure 5 is that an examination of cumulative recall for the low, medium, and high openness groups for the combined tasks (i.e., the three lines in the bottom of Figure 5) revealed a different pattern than for self-reference recall. That is, the high openness group recalled nominally more adjectives from the combined tasks than did the low and medium groups throughout the entire recall period (as opposed to fewer adjectives initially with incremental item gain throughout the recall period). Similar to Study 1, although these data suggest that tapping into the self-concept may be necessary for the relationship between rate of approach and openness to hold, the data should be interpreted with caution. $^{18}$

The zero-order correlations among the memory-based measures and openness from Study 1 (presented in Appendix K) showed that highly open participants recalled more orderliness adjectives than did their less open counterparts $(r=.31, p<.01)$. Furthermore, there was a tendency for highly open participants to gain more items in the second recall task $(r=-.16, p<.20)$ and the rate of approach tended to be slower $(r=-$ $.12, p>.20)$. These findings question the construct validity of the personality-recall speed measure, and thus a closer inspection of cumulative recall was warranted. Figure 6 plots

\footnotetext{
${ }^{18}$ Group level estimates for the best fitting cumulative recall curve (Equation 1) were calculated for the openness groups' combined task performance in Study 2 as presented in Figure 5. The low openness group's asymptote was $\mathrm{n}(\alpha)=2.35(95 \% \mathrm{CI}[2.19,2.52])$ at a rate of $\lambda=.40(95 \% \mathrm{CI}[.30, .49])$; the medium openness group's asymptote was $\mathrm{n}(\alpha)=3.21(95 \% \mathrm{CI}[2.96,3.46])$ at a rate of $\lambda=.22(95 \% \mathrm{CI}$ $[.18, .25])$; and the high openness group's asymptote was $\mathrm{n}(\alpha)=3.44(95 \% \mathrm{CI}[3.22,3.66])$ at a rate of $\lambda=$ $.29(95 \%$ CI $[.24, .34])$. These fits suggest that the medium and high openness groups approached asymptote more slowly than did the low group. Although these results somewhat conflict with predictions of divergent validity, the medium openness group has the slowest rate of approach, not the high openness group.
} 
cumulative recall as a function of low $(n=34)$, medium $(n=38)$, and high $(n=26)$ openness for the self-referenced orderliness adjectives in Study 1.

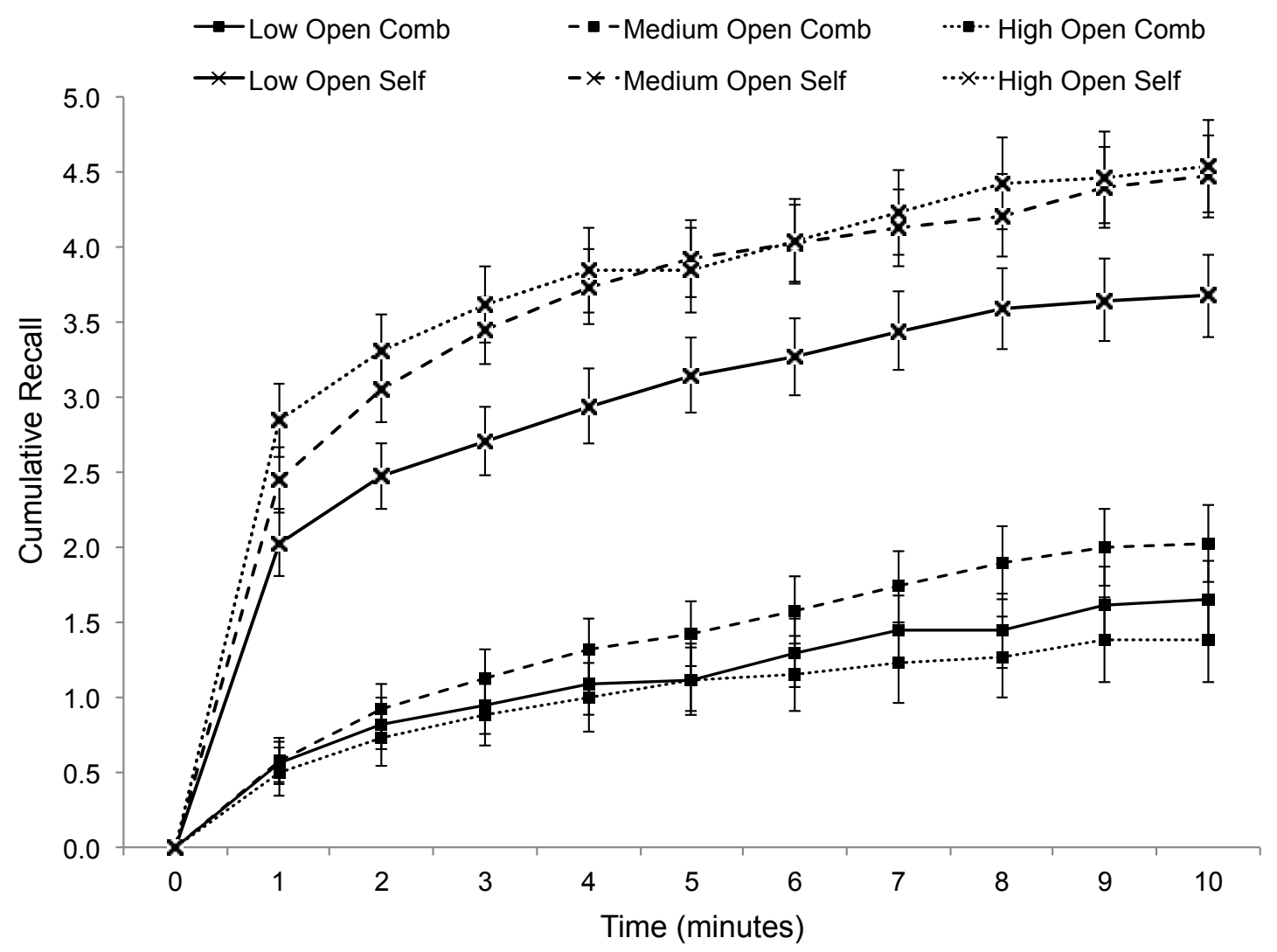

Figure 6. Cumulative recall in Study 1 as a function of openness presented separately for the self-reference task and the combined semantic, rhyming, and structural tasks. Error bars represent standard error of the mean. Open $=$ Openness. Comb $=$ Combined. Self $=$ Self-reference.

The time course of recall for the low and medium openness groups in Study 1 was similar to that observed in Study 2. In particular, Figure 6 shows that the medium openness group recalled slightly more items than the low openness group at minute 1 , the difference between the groups was much larger by minute 3 , and the difference persisted throughout the rest of the recall period. In contrast, there was a striking difference in the time course of recall for the high openness group between the studies. Specifically, recall 
was relatively much slower in Study 2 when the openness adjectives were self-referenced than in Study 1 when the orderliness adjectives were self-referenced. In Study 1, the high openness group recalled more adjectives than the other groups at minute 1, whereas in Study 2 the high openness group recalled fewer adjectives than the other groups at minute 1. Furthermore, the difference in cumulative recall between the low and high openness groups in Study 1 was approximately equivalent throughout the entire recall period (Figure 6), whereas in Study 2 the high openness group recalled nominally fewer adjectives at minute 1 and had incremental item gain until minute 8 when the difference in recall levelled off (Figure 5). This striking difference in recall speed among open participants between Study 1 and 2 along with the null relationships between openness and item loss provide further support for the main tenet of my thesis. That is, participants who were highly open recalled self-referenced adjectives more slowly than their less open counterparts, but that was only the case when the adjectives described openness (Study 2), otherwise a presumably normal time course of recall occurred (i.e., the much faster recall for the highly open participants in Study 1). These results support the elaborative processing explanation, in that the additional item-specific information that was activated when making self-reference decisions about personality congruent adjectives slowed down recall in Study 2, but not in Study1. ${ }^{19,20}$

\footnotetext{
${ }^{19}$ Group level estimates for the best fitting cumulative recall curve (Equation 1) were calculated for the openness groups' combined task performance in Study 1 as presented in Figure 6 . The low openness group's asymptote was $\mathrm{n}(\alpha)=1.67(95 \% \mathrm{CI}[1.46,1.89])$ at a rate of $\lambda=.28(95 \% \mathrm{CI}[.19, .37])$; the medium openness group's asymptote was $\mathrm{n}(\alpha)=2.20(95 \% \mathrm{CI}[1.98,2.42])$ at a rate of $\lambda=.24(95 \% \mathrm{CI}$ $[.18, .29])$; and the high openness group's asymptote was $\mathrm{n}(\alpha)=1.38(95 \%$ CI $[1.29,1.47])$ at a rate of $\lambda=$ $.35(95 \%$ CI $[.28, .42])$. These fits suggest that the high openness group approached asymptote more quickly, not slowly, than did the low and medium openness groups (who did not differ from one another).

${ }^{20}$ Group level estimates for the best fitting cumulative recall curve (Equation 1) were calculated for the openness groups' self-reference task performance in Study 1 as presented in Figure 6. The low openness group's asymptote was $\mathrm{n}(\alpha)=3.48(95 \%$ CI $[3.25,3.72])$ at a rate of $\lambda=.62(95 \%$ CI $[.43, .80])$; the medium openness group's asymptote was $\mathrm{n}(\alpha)=4.21(95 \%$ CI $[4.00,4.41])$ at a rate of $\lambda=.68(95 \% \mathrm{CI}$
} 
A final note about cumulative recall in Study 2 is that imaginativeness may not have correlated significantly with the rate of approach parameter because an unusual combination of item-specific and relational information was driving recall. To explore that possibility, Figure 7 below presents cumulative recall separately for participants who reported low $(n=33)$, medium $(n=30)$, or high $(n=27)$ imaginativeness. Unlike what has been seen thus far, participants who were highly imaginative recalled self-referenced items very quickly in the first recall task, but started to approach asymptote toward the end of the first recall task (i.e., at minute 5), signifying the use of relational information (in contrast to what the item loss results presented in Table 8 suggested, which was that imaginativeness was marginally related to more item loss; i.e., less relational information). However, in the second recall task, they also gained more items than those who scored low (and to a less extent, medium) on imaginativeness, signifying the use of additional item-specific information.

$[.52, .83])$; and the high openness group's asymptote was $\mathrm{n}(\alpha)=4.21(95 \% \mathrm{CI}[3.96,4.46])$ at a rate of $\lambda=$ $.87(95 \%$ CI $[.58,1.16])$. These fits suggest that the high openness group approached asymptote more quickly, not slowly, than did the low and medium openness groups (who did not differ from one another). 


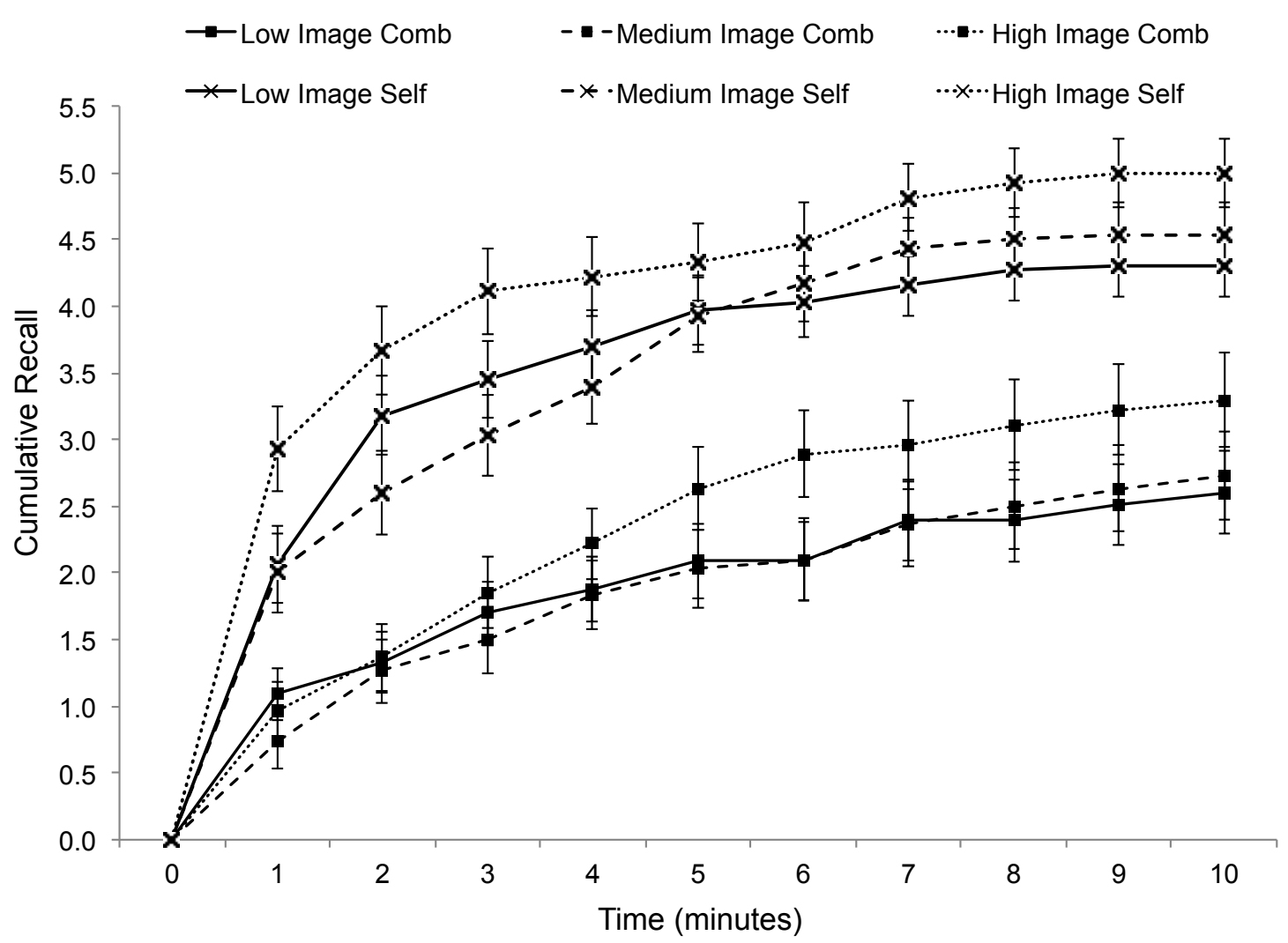

Figure 7. Cumulative recall in Study 2 as a function of imaginativeness presented separately for the self-reference and combined semantic, rhyming, and structural tasks. Error bars represent standard error of the mean. Image $=$ Imaginativeness. $\mathrm{Comb}=$ Combined. Self $=$ Self-reference.

Divergent Validity. A second set of correlations that included the direct measures of conscientiousness and orderliness were run to examine whether the results from Study 1 were due to the careful (and perhaps slow) nature of people who are highly conscientious and orderly. If the main results from Study 1 were due to conscientious or orderly behaviour per se and not the elaboration of adjectives when making self-reference decisions, I hypothesized that the direct measures of conscientiousness and orderliness would be significantly negatively related to the rate of approach and positively related to item gain in Study 2. Table 9 shows that conscientiousness and orderliness were not 
associated with the rate of approach, nor were they associated with encoding-openness, openness recall, correct recognition, the openness-IAT, item gain, or item loss. There was a significant negative association between orderliness and false recognition, wherein orderly participants falsely recognized fewer openness lures. These results provide evidence that the main cumulative recall findings of Study 1 were not due to conscientious and orderly behaviour per se.

Table 9

Zero-Order Correlations Among Direct and Indirect Personality Measures and SelfReference Memory Processes

\begin{tabular}{lcc}
\hline \multicolumn{1}{c}{ Measure } & 1 & 2 \\
\hline 1. Conscientiousness & - & \\
2. Orderliness & $.44^{* * *}$ & - \\
3. Encoding-Openness & .04 & -.07 \\
4. Openness Recall & .00 & -.01 \\
5. Rate of Approach & -.14 & .03 \\
6. Correct Recognition & -.08 & .02 \\
7. False Recognition & -.05 & $-.21^{*}$ \\
8. Openness-IAT & -.09 & -.06 \\
9. Inverse Item gain ${ }^{\text {a }}$ & .06 & .10 \\
10. Inverse Item loss ${ }^{\text {a }}$ & .01 & -.09 \\
\hline
\end{tabular}

Note: $d f \mathrm{~s}=88$ to 90 .

${ }^{a}$ Inverse transformed. Must be interpreted accordingly.

${ }^{++} p<.20 .{ }^{+} p<.10 .{ }^{*} p<.05 .{ }^{* *} p<.01 .{ }^{* * *} p<.001$. 
To corroborate the rate of approach and item gain results presented in Table 9, Figure 8 plots cumulative recall for participants who were low $(n=31)$, medium $(n=30)$, or high $(n=29)$ on the direct measure of orderliness. The time course of recall for the self-referenced adjectives (i.e., the top three lines in Figure 8) was similar between the high and low orderliness groups, with the low orderliness group recalling nominally more adjectives at minute 1 , the high orderliness group recalling nominally more adjectives at minute 2 , followed by a convergence of their cumulative recall from minute 3 onwards. The medium orderliness group had the lowest level of cumulative recall from minute 3 onwards. Clearly, the high orderliness group did not recall the self-referenced orderliness adjectives more slowly than did the low and medium groups. ${ }^{21}$ It was particularly illuminating to compare the pattern of cumulative recall in Figure 8 to that observed in Study 1 (Figure 3), where the rate of approach was negatively associated with orderliness. Specifically, in Study 1, the high orderliness group was clearly slower to recall the orderliness adjectives than were the low and medium orderliness groups, whose time course of recall was almost identical. An examination of cumulative recall in Study 2 as a function of orderliness corroborates the null relationships between orderliness and rate of approach and item gain in Table 9. These results support the divergent validity of the rate of approach measure of orderliness under self-reference encoding. ${ }^{22}$

\footnotetext{
${ }^{21}$ Group level estimates for the best fitting cumulative recall curve (Equation 1) were calculated for the orderliness groups' self-reference task performance in Study 2 as presented in Figure 8. The low orderliness group's asymptote was $\mathrm{n}(\alpha)=4.56(95 \% \mathrm{CI}[4.32,4.81])$ at a rate of $\lambda=.59(95 \% \mathrm{CI}[.45$, $.73])$; the medium orderliness group's asymptote was $\mathrm{n}(\alpha)=4.12(95 \% \mathrm{CI}[3.82,4.41])$ at a rate of $\lambda=.53$ $(95 \% \mathrm{CI}[.38, .69])$; and the high orderliness group's asymptote was $\mathrm{n}(\alpha)=4.75(95 \% \mathrm{CI}[4.60,4.90])$ at a rate of $\lambda=.60(95 \% \mathrm{CI}[.52, .68])$. These fits suggest that the rate of approach was similar among the orderliness groups. Clearly, the high orderliness group did not recall the self-referenced openness adjective more slowly than the medium and low groups, as they did in Study 1 when the adjectives described orderliness, which supports divergent validity.

${ }^{22}$ Group level estimates for the best fitting cumulative recall curve (Equation 1) were also calculated for the orderliness groups' combined task performance in Study 2 as presented in Figure 8. The low orderliness
} 


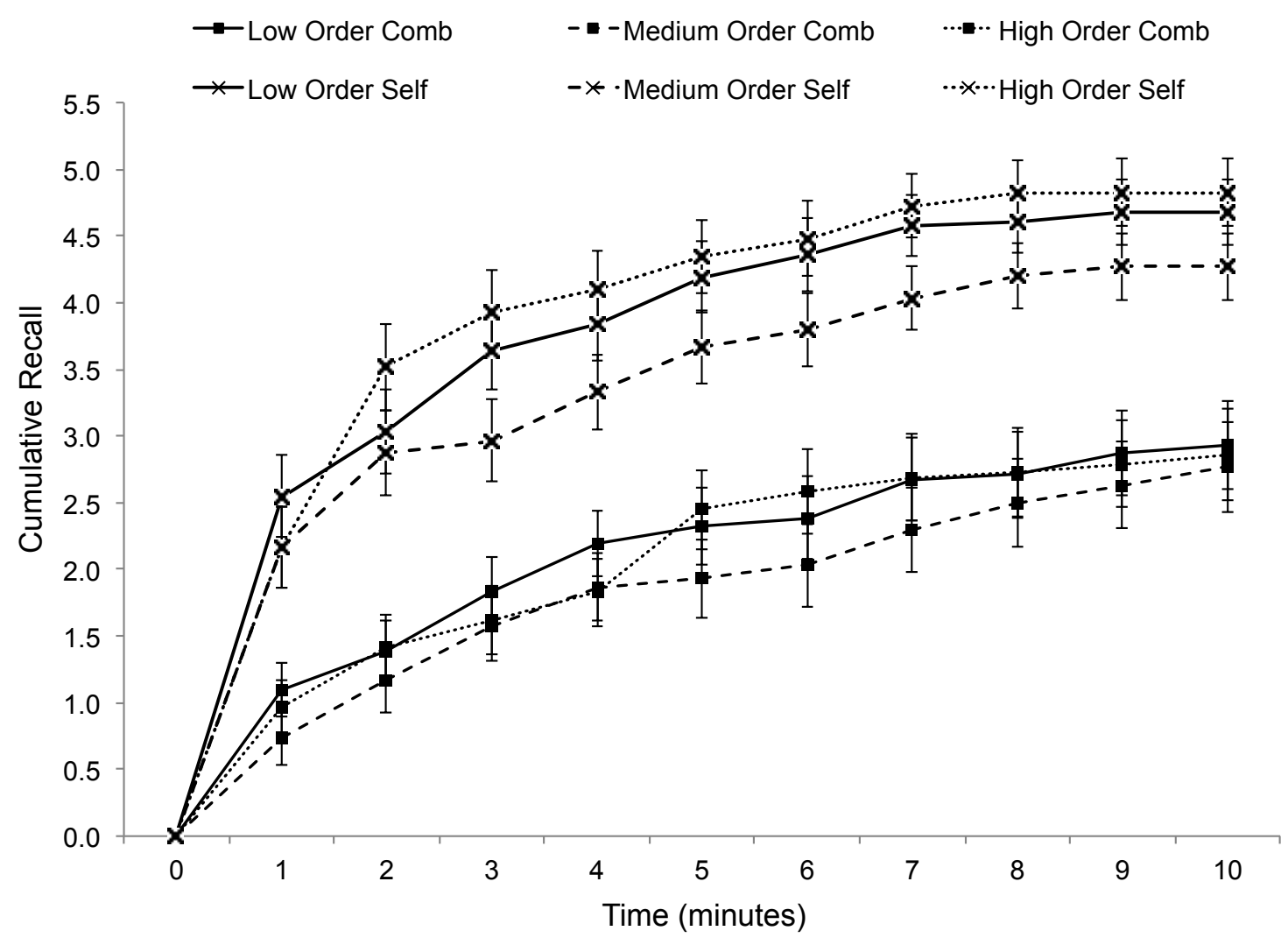

Figure 8. Cumulative recall in Study 2 as a function of orderliness presented separately for the self-reference and combined semantic, rhyming, and structural tasks. Error bars represent standard error of the mean. Order $=$ Orderliness. Comb $=$ Combined. Self $=$ Self-reference.

Self-reported and observed behaviour. The self-report items that have been shown to relate to a direct measure of openness across a variety of different openness scales (i.e., reading classic literature, playing a musical instrument, general intelligence, and pursuing a liberal arts degree; Paunonen, 2003) were included in analyses. An item asking whether participants would want their romantic partner to be open to new

group's asymptote was $\mathrm{n}(\alpha)=2.95(95 \%$ CI $[2.74,3.16])$ at a rate of $\lambda=.33(95 \%$ CI $[.26, .40])$; the medium orderliness group's asymptote was $\mathrm{n}(\alpha)=2.92(95 \% \mathrm{CI}[2.62,3.22])$ at a rate of $\lambda=.24(95 \% \mathrm{CI}$ $[.18, .30])$; and the high orderliness group's asymptote was $\mathrm{n}(\alpha)=3.04(95 \%$ CI $[2.74,3.34])$ at a rate of $\lambda$ $=.29(95 \%$ CI $[.21, .36])$. These fits suggest that the medium orderliness group approached asymptote more slowly than did the low, and to a lesser extent, the high orderliness group (whereas the medium and high groups did not differ from one another), supporting divergent validity. 
experiences and one asking the probability of voting conservative were also included.

The daydreaming task administered in the laboratory provided a measure of openness behaviour (i.e., time spent daydreaming) and self-reported vividness of the daydream. Additionally, whether participants decided to take the debriefing sheet and whether they exhibited any spontaneous curiosities during the experimental session were examined as openness behaviour.

Before computing correlations, the measures were assessed for normality and outliers using the criteria adopted in previous sections. Daydreaming duration was positively skewed and kurtotic, but a log transformation rendered a normal distribution without outliers. Self-reported general intelligence had one outlier $(z=-3.73)$. In the following analyses, the encoding-openness, false recognition, and item gain outliers identified in the previous section were also excluded when the respective variable or combination of variables were involved in a particular correlation. Table 10 presents the zero-order correlations among the personality and memory-based measures and the measures of openness attitudes and behaviours.

Table 10

Zero-Order Correlations Between Direct and Indirect Personality Measures and Openness Self-Reports and Behaviour

\begin{tabular}{lccccc}
\hline \multicolumn{1}{c}{$\begin{array}{c}\text { Personality } \\
\text { Measure }\end{array}$} & $\begin{array}{c}\text { Daydream } \\
\text { Duration }\end{array}$ & $\begin{array}{c}\text { Daydream } \\
\text { Vividness }\end{array}$ & $\begin{array}{c}\text { General } \\
\text { Intelligence }\end{array}$ & $\begin{array}{c}\text { Romantic } \\
\text { Partner }\end{array}$ & $\begin{array}{c}\text { Voting } \\
\text { Conservative }\end{array}$ \\
\hline 1. Openness & .13 & .07 & .06 & $.23^{*}$ & $-.37^{* * *}$ \\
2. Imaginativeness & $.17^{++}$ & $.26^{*}$ & -.02 & $.22^{*}$ & -.06 \\
$\begin{array}{l}\text { 3. Encoding- } \\
\text { Openness }\end{array}$ & .11 & $.23^{*}$ & .06 & .11 & $-.22^{*}$
\end{tabular}




\begin{tabular}{|c|c|c|c|c|c|}
\hline 4. Openness Recall & $.23^{*}$ & .12 & $.21^{*}$ & .08 & $-.16^{++}$ \\
\hline $\begin{array}{l}\text { 5. Rate of } \\
\text { Approach }\end{array}$ & -.11 & .00 & .02 & -.12 & $.28^{* *}$ \\
\hline $\begin{array}{l}\text { 6. Correct } \\
\text { Recognition }\end{array}$ & $.24^{*}$ & -.01 & .05 & .04 & .00 \\
\hline $\begin{array}{l}\text { 7. False } \\
\text { Recognition }\end{array}$ & -.01 & .07 & $-.18^{++}$ & .02 & -.03 \\
\hline 8. Openness-IAT & .03 & -.12 & .06 & .13 & .08 \\
\hline $\begin{array}{l}\text { 9. Inverse Item } \\
\text { gain }^{\text {a }}\end{array}$ & .11 & -.10 & $.27^{*}$ & -.11 & .06 \\
\hline $\begin{array}{l}\text { 10. Inverse Item } \\
\operatorname{loss}^{\text {a }}\end{array}$ & -.04 & -.02 & .03 & .13 & -.03 \\
\hline
\end{tabular}

Note. $n=85$ to 90 . Two-tailed tests.

${ }^{a}$ Inverse transformed. Must be interpreted accordingly. ${ }^{++} p<.20 .{ }^{+} p<.10 .{ }^{*} p<.05 .^{* * *} p<.01 .^{* * * *} p<.001$.

Concerning in-lab openness behaviour, the results show that imaginativeness was marginally associated with longer daydreaming duration. Furthermore, higher openness recall and correct recognition were significantly associated with longer daydreaming duration. Although rate of approach to asymptote and item gain were associated with longer daydream duration in the predicted direction, the correlations did not reach statistical significance. These results provide preliminary support for daydreaming duration as a valid measure of imaginative behaviour. They also suggest that the itemspecific information elicited from the self-reference task that gave rise to higher openness recall and correct recognition were predictive of an in-lab measure of openness behaviour. Although imaginativeness and encoding-openness were significantly associated with higher daydreaming vividness, the other direct, indirect, and memory- 
based measures were not associated with daydreaming vividness. Further results of in-lab behaviour presented in Table 11 showed that participants who decided to take the debriefing sheet at the end of the experimental session were significantly more open to experience $\left(M_{\text {diff }}=-.37, t(86)=-2.59, p=.01\right)$, they gained more openness adjectives in the second recall task $\left(M_{\text {diff }}=.04, t(78.72)=2.76, p=.007\right)$, they falsely recognized more openness lures $\left(M_{\text {diff }}=-.07, t(83)=-2.05, p=.04\right)$, and they endorsed more openness adjectives as self-descriptive in the self-reference task $\left(M_{\text {diff }}=-.93, t(84)=-\right.$ $2.18, p=.03)$. Participants who showed spontaneous curiosity about the study during the experimental session were more highly open to experience $\left(M_{\text {diff }}=-.35, t(87)=-2.39, p=\right.$ $.02)$ and they tended to correctly recognize more openness adjectives $\left(M_{\text {diff }}=-.02\right.$, $t(68.68)=-1.64, p=.11)$ than those who did not show any curiosity. Although not all personality and memory-based measures predicted in-lab behaviours, the results provide some degree of support for the construct validity of the memory-based measures.

Table 11

Means and Standard Deviations of the Direct and Indirect Personality Measures as a Function of Openness Behaviour in Study 2

\begin{tabular}{lcccc}
\hline & \multicolumn{2}{c}{ Debriefing Sheet } & \multicolumn{2}{c}{ Spontaneous Curiosity } \\
\hline \multicolumn{1}{c}{ Personality Measure } & No $(\mathrm{n}=31)$ & Yes $(\mathrm{n}=57)$ & No $(\mathrm{n}=62)$ & Yes $(\mathrm{n}=27)$ \\
\hline 1. Openness & $3.37(.65)$ & $3.74(.64)^{*}$ & $3.48(.64)$ & $3.84(.65)^{*}$ \\
2. Imaginativeness & $3.56(.58)$ & $3.76(.61)^{++}$ & $3.64(.62)$ & $3.79(.57)$ \\
3. Encoding-Openness & $5.83(1.88)$ & $6.77(1.91)^{*}$ & $6.55(1.91)$ & $6.46(2.04)$ \\
4. Openness Recall & $4.65(1.62)$ & $4.56(1.23)$ & $4.52(1.36)$ & $4.81(1.33)$ \\
5. Rate of Approach & $.33(.28)$ & $.33(.33)$ & $.34(.33)$ & $.30(.26)$ \\
6. Correct Recognition & $.97(.07)$ & $.95(.07)$ & $.95(.07)$ & $.97(.05)^{+}$ \\
7. False Recognition & $.18(.14)$ & $.25(.17)^{*}$ & $.22(.15)$ & $.23(.18)$
\end{tabular}




$\begin{array}{lcccc}\text { 8. Openness-IAT } & .47(.25) & .48(.24) & .47(.24) & .46(.23) \\ \text { 9. Inverse Item gain }{ }^{\text {a }} & .98(.03)^{* *} & .96(.05) & .97(.05) & .97(.05) \\ \text { 10. Inverse Item loss }{ }^{\text {a }} & .92(.11) & .93(.10) & .92(.11) & .94(.09)\end{array}$

Note. $d f=84$ to 87 . Two-tailed tests.

${ }^{a}$ Inverse transformed. Must be interpreted accordingly. ${ }^{++} p<.20 .{ }^{+} p<.10 .^{*} p<.05 .^{* *} p<.01$.

Table 12 displays the correlations among self-reported attitudes and behaviours that have been shown to relate to openness in past research. The results show that higher openness recall was significantly associated with higher self-reported general intelligence, and less false recognition tended to be associated with higher self-reported general intelligence. In contrast, although item gain was significantly associated with general intelligence, the association was not in the predicted direction (i.e., higher item gain was hypothesized to be associated with higher general intelligence). Further results showed that the direct measures of openness and imaginativeness were related to participants' preference for a romantic partner who is open to experience, but was not associated with the other indirect and memory-based measures. As was predicted, directly measured openness, encoding-openness, higher openness recall (approaching marginal), and slower recall speed were significantly associated with a lower probability of voting for the conservative party. Participants who indicated that they enjoy reading classic literature were more highly open to experience $\left(M_{\text {diff }}=-.37, t(88)=-2.63, p=.01\right)$ and gained marginally more openness adjectives in the second recall task $\left(M_{\text {diff }}=.02\right.$, $t(49.02)=1.56, p=.13)$. Participants who reported that they play a musical instrument were more open to experience than those who reported that they do not play an instrument $\left(M_{\text {diff }}=-.29, t(88)=-2.12, p=.04\right)$. Participants who indicated that they are 
enrolled, or have been enrolled, in a liberal arts program recalled the openness adjectives more slowly over the recall period $\left(M_{\text {diff }}=-.17, t(33.04)=-2.07, p=.05\right)$ and gained more items in the second recall task $\left(M_{\text {diff }}=-.03, t(61.53)=-3.00, p=.004\right)$ than did those who were enrolled in a non-liberal arts program. 
Table 12

Means and Standard Deviations of the Direct and Indirect Personality Measures as a Function of Self-Reported Openness Attitudes and Behaviours in Study 2

\begin{tabular}{|c|c|c|c|c|c|c|}
\hline \multirow[b]{2}{*}{ Personality Measure } & \multicolumn{2}{|c|}{ Classic Literature } & \multicolumn{2}{|c|}{ Musical Instrument } & \multicolumn{2}{|c|}{ Liberal Arts Degree } \\
\hline & $\begin{array}{c}\text { No } \\
(n=59)\end{array}$ & $\begin{array}{c}\text { Yes } \\
(n=31)\end{array}$ & $\begin{array}{c}\text { No } \\
(n=53)\end{array}$ & $\begin{array}{c}\text { Yes } \\
(n=37)\end{array}$ & $\begin{array}{c}\text { No } \\
(n=25)\end{array}$ & $\begin{array}{c}\text { Yes } \\
(n=65)\end{array}$ \\
\hline 1. Openness & $3.47(.69)$ & $3.84(.53)^{*}$ & $3.48(.62)$ & $3.77(.68)^{*}$ & $3.69(.76)$ & $3.57(.62)$ \\
\hline 2. Imaginativeness & $3.65(.65)$ & $3.75(.52)$ & $3.61(.59)$ & $3.79(.61)^{++}$ & $3.76(.70)$ & $3.66(.56)$ \\
\hline 3. Encoding-Openness & $6.37(2.02)$ & $6.75(1.78)$ & $6.34(2.11)$ & $6.75(1.65)$ & $6.71(2.13)$ & $6.43(1.87)$ \\
\hline 4. Openness Recall & $4.51(1.43)$ & $4.74(1.21)$ & $4.51(1.48)$ & $4.70(1.18)$ & $4.72(1.37)$ & $4.54(1.36)$ \\
\hline 5. Rate of Approach & $.31(.31)$ & $.36(.31)$ & $.34(.32)$ & $.30(.29)$ & $.45(.38)^{*}$ & $.28(.26)$ \\
\hline 6. Correct Recognition & $.95(.08)$ & $.96(.06)$ & $.96(.07)$ & $.96(.06)$ & $.96(.07)$ & $.96(.06)$ \\
\hline 7. False Recognition & $.22(.15)$ & $.25(.17)$ & $.22(.16)$ & $.23(.17)$ & $.20(.14)$ & $.24(.17)$ \\
\hline 8. Openness-IAT & $.47(.25)$ & $.47(.21)$ & $.47(.21)$ & $.47(.28)$ & $.48(.23)$ & $.45(.27)$ \\
\hline 9. Inverse Item gain ${ }^{\text {a }}$ & $.97(.04)^{++}$ & $.96(.05)$ & $.97(.05)$ & $.97(.05)$ & $.99(.03)^{* *}$ & $.96(.05)$ \\
\hline 10. Inverse Item loss ${ }^{a}$ & $.93(.10)$ & $.93(.11)$ & $.92(.11)$ & $.94(.10)$ & $.93(.10)$ & $.93(.11)$ \\
\hline
\end{tabular}

Note. $d f=86$ to 88 . Two-tailed tests.

${ }^{\text {a }}$ Inverse transformed. Must be interpreted accordingly.

${ }^{++} p<.20 .{ }^{+} p<.10 .{ }^{*} p<.05 .^{* * *} p<.01$. 
Aside from the item gain and general intelligence correlation, the other relationships that surfaced as marginally significant or statistically significant were in the predicted direction. Given that the ten measures of openness attitudes and behaviours were examined against ten personality and memory-based measures (i.e., one-hundred inferential tests), five relationships were expected to surface as statistically significant even though the relationships did not exist in the sampled population (i.e., five Type 1 errors were expected). Out of all tests that were conducted, nineteen surfaced as statistically significant at $\alpha=.05$, and all except for one were in the predicted direction. The main relationships of interest were concerning personality measures and recall speed (rate of approach and item gain). Of these, four of twenty relationships surfaced as statistically significant in the predicted direction (the in-lab behaviour of taking the debriefing sheet, voting conservative, and taking a liberal arts program), whereas only two would be expected by chance. In sum, the memory-based personality measures were associated with some in-lab and self-reported measures of openness behaviour, however effect sizes were small and recall speed and item gain were not consistently associated with the behaviour criteria. Hence, the predictive validity was fairly weak overall.

\section{Discussion}

The significant negative openness-recall speed correlation and an examination of cumulative recall support the main tenet of my thesis, which is that self-reference encoding of trait adjectives taps a structured aspect of self-concept through elaborative processing. In particular, the Study 2 results suggest that highly open participants engaged in more elaborate processing when making self-reference decisions of openness adjectives relative to those who were lower on that trait, resulting in more item-specific 
information to aid recall, but slowing down recall speed. Item loss in the second recall trial was not associated with openness or recall speed, suggesting that the significant openness-recall speed association was not due to relational information acquired through organizational processing. An examination of a plot of cumulative recall supported these conclusions.

These results demonstrated a conceptual replication of the Study 1 findings that conscientiousness and orderliness were related to slower recall speed of corresponding self-referenced adjectives, and were not related to item loss in the second recall trial. Importantly, further correlational analysis and examination of cumulative recall uncovered that conscientiousness and orderliness in Study 2 were not related to recall speed of the self-referenced openness adjectives, which cast doubt on the alternative explanation that conscientious and orderly people simply approach the task more carefully (i.e., slowly). Furthermore, additional analysis and examination of cumulative recall uncovered that openness was not associated with recall speed of the self-referenced orderliness adjectives in Study 1. Hence, between the two studies, there was an uncrossed double dissociation in self-reference recall speed between the target (and non-target) traits supporting convergent and divergent validity, and suggesting personality congruence may be necessary to observe the hypothesized relationships. Furthermore, group-level estimates of cumulative recall curve parameters presented in footnotes not only supported the uncrossed double dissociation noted above, but also suggested that recall performance in the combined tasks was not related to participants' standing on the target and (non-target) traits in the same way, supporting divergent validity (i.e., the hypothesized personality-recall speed associations were only observed under self- 
reference encoding, suggesting the self-concept must be tapped to observe the relationships). The self-report measures of personality in Study 2 were taken during the Fall 2012 mass-testing session at the start of the academic term instead of at the end of the experimental session, which reduced the impact of demand characteristics on the relationship between the direct personality measures and recall speed.

While Study 2 was a successful conceptual replication and extension of Study 1, some results did not replicate and there were some unexpected findings. In particular, the trends noted in Study 1 among the direct measures of personality and false recognition and the orderliness-IAT were negligible in Study 2. Hence, the additional item-specific information elicited by the self-reference task among people high on a corresponding trait did not seem to consistently prevent false recognition, and the item-specific information did not seem to be akin to the relative strength of associations contained in the implicit personality self-concept, but instead seemed to be akin to the propositions of the explicit personality self-concept. Although the overall level of recall of self-referenced adjectives in Study 1 was not related to orderliness or conscientiousness, openness and imaginativeness in Study 2 tended to be associated with a higher proportion recalled. It is also important to note that openness in Study 1 was significantly associated with higher recall of the orderliness adjectives (but not recall speed).

It is possible that the higher recall among open and imaginative participants was trait specific, in that there is something about people who are open to experience and imaginative that leads them to encode more information, especially in a self-reference task. In support of this idea, the observed results in Study 2 were similar to that of Katz (1989), who found that creativity was related to higher levels of recall of creative- 
relevant adjectives following a self-reference task, but not to creative-irrelevant adjectives encoded in a self-reference task or to the adjectives presented in other encoding tasks. However, unlike what Katz (1989) found, it should be noted that orderliness recall in Study 1 was significantly positively related to openness and imaginativeness, suggesting that open and imaginative people recall more adjectives whether the adjectives match the trait (i.e., openness) or not (i.e., orderliness). An examination of cumulative recall in Study 2 suggested that the medium openness group encoded more relational information than did the low openness group (i.e., the recall difference was fully realized by minute 2 ). Furthermore, examination of cumulative recall in Study 1 as a function of openness suggested that participants who reported low levels of openness encoded less relational information than did those who were higher on the trait (i.e., the recall difference was mostly evidenced at minute 1 as opposed to a slowly increasing difference over the recall period). Unfortunately, the item loss data in Study 1 and 2 did not support the idea that openness was related to processing more relational information (i.e., the item loss and direct personality correlations were null).

Of particular note in Study 2 was the different pattern of results that emerged for the imaginativeness facet, with those high on the facet gaining and losing marginally more adjectives in the second recall trial than those low on the facet. Examination of cumulative recall suggested that participants who were highly imaginative engaged in more relational and item-specific processing than those who were less imaginative. Specifically, highly imaginative participants' recall was fast early in the first recall trial, indicating the use of effective relational information to narrow the search of memory, but there also tended to be higher levels of item gain during the second recall trial, indicating 
that the encoded item-specific information had not been exhausted toward the end of the recall period. It is possible that the unusual combination of item-specific and relational information obscured the relationship between imaginativeness and recall speed. It should be noted, however, that the item loss results showed that highly imaginative people lost marginally more items in the second recall trial, suggesting that any relational information that was encoded by organizational processing produced a less effective retrieval plan (which is contrary to the pattern of cumulative recall observed in Figure 7). Given that openness is a very broad construct encompassing a wide range of attitudes (e.g., political, intellectual, religious; McCrea \& Sutin, 2007) whereas imaginativeness is quite specific in that it describes those who engage in fantasy and innovative thinking, it is possible that open and imaginative participants processed the openness adjectives differently in the self-reference task. For example, highly imaginative participants may have noticed and explored the categorical relations among the items as a way to engage fantasy and creative thinking even more so than did open people, which would explain the high level of relational processing observed in the early portion of cumulative recall broken down by imaginativeness (but would not explain the item loss data).

\section{Study 3}

Study 3 replicated and extended the previous two studies by implementing the other-reference task in addition to the self-reference task in a within-subjects design. Participants encoded the orderliness adjectives by making other-reference judgments (i.e., "Describes my best friend?") and the imaginativeness adjectives by making self-reference judgments (i.e., same as in Study 2). In addition, participants completed the semantic orienting task on a new list of adjectives describing trust, a facet of agreeableness 
(Goldberg et al., 2006). They also completed direct personality measures for themselves as well as for the other-referent individual. The openness-IAT from Study 2 was implemented to obtain an indirect measure of participants' openness. Other-reference recall and recognition performance tested the generality of the relationship between elaborative processing and personality by tapping into a memory structure other than personality self-concept. Study 3 was therefore designed to determine whether the steepness of recall curves (and false recognition) following other-reference encoding of orderliness adjectives relates to the other-referents' orderliness (but not the participants' orderliness). Furthermore, it simultaneously tested whether the steepness of recall curves (and false recognition) following self-reference encoding of imaginativeness adjectives relates to the participants' imaginativeness (but not the other-referents' imaginativeness; i.e., an uncrossed double dissociation in recall speed between the tasks and traits). Lastly, predictive validity of the memory-based measures was tested in the same way as in the previous two studies, with additional questions designed to measure the other-referents' trait-related attitudes and behaviours. Given that the other-reference and self-reference tasks were expected to produce relatively high levels of recall, and very elaborate processing for some participants, the duration of the two recall tasks was increased to 7 minutes each, for a total of 14 minutes of recall.

\section{Method}

Participants. One hundred and sixty three undergraduate psychology students who were not tested in the previous studies were recruited from SONA. One hundred and nine participants were female $(66.9 \%)$. 


\section{Materials.}

Friend questionnaire. A one-page questionnaire was developed to assess the closeness of the participant and their selected friend and to help ensure a friend was brought to mind (see Appendix M). The questionnaire asked for the friend's first name, how frequently they communicate with their friend (Daily, Weekly, Monthly, or Yearly), how many years they have known their friend (open-ended question), how well they know their friend (using a seven point Likert-type scale from 1 [Not Well] to 7 [Extremely Well]), and how close they are with their friend (based on Aron, Aron, \& Smollan's, 1992 inclusion of other in the self scale).

Levels-of-processing. The orderly and imaginative lists from Study 2 were combined with a new list of adjectives representing the positive pole of trust, a facet of agreeableness (Goldberg et al., 2006). The trust adjectives were selected, using the same approach from Study 2, to maximally separate the lists in latent semantic space to provide a relational cue and improve recall. Each list was composed of 13 adjectives to keep the number of trials among the studies approximately the same. The orderly and imaginative lists from the previous studies were therefore supplemented with three new adjectives each (see Table 13).

Table 13

Levels-of-Processing Items for the Trust, Imaginativeness, and Orderliness Facets

\begin{tabular}{ccc}
\hline & Memory List & \\
Trust & Orderliness & Imaginativeness \\
\hline Trusting & Punctual & Curious \\
Honest & Organized & Eccentric \\
Loyal & Efficient & Visionary
\end{tabular}




$\begin{array}{ccc}\text { Generous } & \text { Particular } & \text { Deep } \\ \text { Principled } & \text { Careful } & \text { Artistic } \\ \text { Sympathetic } & \text { Strict } & \text { Inventive } \\ \text { Friendly } & \text { Precise } & \text { Poetic } \\ \text { Cooperative } & \text { Accurate } & \text { Imaginative } \\ \text { Humane } & \text { Measured } & \text { Creative } \\ \text { Gentle } & \text { Prompt } & \text { Reflective } \\ \text { Agreeable } & \text { Systematic } & \text { Romantic } \\ \text { Compassionate } & \text { Reliable } & \text { Inspired } \\ \text { Faithful } & \text { Thorough } & \text { Gifted }\end{array}$

Note. Items in bold have been used in previous research on agreeableness-, conscientiousness-, or openness-IATs.

The three lists were equated in terms of word frequency, word length, and meaningfulness. The trust adjectives had a tendency to be more likeable than the orderly and imaginative adjectives, but the latter two did not differ in likeableness. ${ }^{23}$ More importantly, the latent semantic association of the lists with their respective trait concept (e.g., the trust adjectives with the trust concept) was significantly higher than its latent semantic association to the other concepts (e.g., the trust list with the orderly and imaginative concepts; see Table 14). The other-reference orienting question asked "Describes my best friend?" and the semantic and self-reference tasks were the same as those used in Study 1 and 2.

\footnotetext{
${ }^{23}$ The trust adjectives were encoded in the semantic task and therefore were expected to be poorly recalled despite additional likeableness.
} 
Table 14

Mean Psycholinguistic Properties for the Trust, Imaginativeness, and Orderliness Memory Lists

\begin{tabular}{ccccc}
\hline & Trust & Orderly & Imaginative & $F$ \\
\hline Word Frequency & $2.43(.67)$ & $2.44(.59)$ & $2.41(.64)$ & .01 \\
Word Length & $8.38(2.36)$ & $8.00(1.29)$ & $7.92(1.85)$ & .22 \\
Likeableness $^{\mathrm{a}}$ & $494.33^{\mathrm{b}}(41.26)$ & $424.20^{\mathrm{c}}(73.89)$ & $419.29^{\mathrm{d}}(76.93)$ & $3.60^{*}$ \\
Meaningfulness $^{\mathrm{a}}$ & $367.78^{\mathrm{b}}(15.54)$ & $361.60^{\mathrm{c}}(19.46)$ & $355.71^{\mathrm{d}}(19.77)$ & 1.26 \\
Imaginative LSA & $.14(.05)$ & $.12(.06)$ & $.29(.12)$ & $15.23^{* * *}$ \\
Orderliness LSA & $.09(.05)$ & $.22(.09)$ & $.09(.03)$ & $17.24^{* * *}$ \\
Trust LSA & $.22(.06)$ & $.09(.04)$ & $.10(.05)$ & $26.76^{* * *}$ \\
\hline
\end{tabular}

Note. Standard deviation is presented within parentheses.

${ }^{\mathrm{a}}$ Calculated from Anderson's (1968) personality trait norms. ${ }^{\mathrm{b}}$ missing data for principled, humane, compassionate, and faithful. ${ }^{\mathrm{c}}$ missing data for organized, particular, and measured. ${ }^{\mathrm{d}}$ missing data for poetic, deep, reflective, visionary, inspired, and gifted. ${ }^{*} p<.05{ }^{* * *} p<.001$.

Working memory. The same as in Study 1 and 2.

Recognition memory. In addition to the 39 old orderly, imaginative, and trust adjectives, 39 lures were carefully selected using the same procedure as in Study 2. Specifically, 13 adjectives for each facet that maximally separated the trait concepts in latent semantic space were selected. The 20 unrelated items were the same as those used in Study 2. The recognition lures for the three facets were equated in terms of word frequency, word length, and meaningfulness. The latent semantic association of the lists with their respective trait concept (e.g., trust list with the trust concept) was significantly higher than with the other trait concepts (e.g., trust list with the orderliness and 
imaginativeness concepts). Appendix $\mathrm{N}$ presents the lures and descriptive and inferential statistics for word frequency, word length, meaningfulness, likeableness, LSA to the trust concept, LSA to the orderliness concept, and LSA to the imaginativeness concept.

Implicit Association Test. The imaginativeness-IAT from Study 2 was administered.

Personality self-report. The 50 IPIP items measuring conscientiousness $(\alpha=.80)$, orderliness $(\alpha=.76)$, openness $(\alpha=.78)$, extraversion $(\alpha=.84)$, and neuroticism $(\alpha=.84)$ from Study 1 were combined with the 9 unique IPIP items measuring imaginativeness ( $\alpha$ $=.81)$ from Study 2 . An additional 20 IPIP items measuring agreeableness $(\alpha=.74)$ and trust $(\alpha=.84)$ were included to yield a 79-item personality questionnaire.

Personality other-report. The phrasing of instructions and items in the personality self-report questionnaire were altered to refer to the participants' friend. Scale reliabilities were as follows: conscientiousness $(\alpha=.89)$, orderliness $(\alpha=.89)$, openness $(\alpha=.81)$, extraversion $(\alpha=.89)$, neuroticism $(\alpha=.74)$, imaginativeness $(\alpha=.83)$, agreeableness $(\alpha$ $=.84)$, and trust $(\alpha=.88)$.

Behaviour self-report. The questionnaire used in Study 2 was administered.

Behaviour other-report. An adaptation of the behaviour self-report questionnaire used in Study 2 was administered. The phrasing of the instructions and questions were directed toward the participants' friend.

Design. The levels-of-processing task was a one-way (encoding task: semantic, other-reference, self-reference) repeated-measures design.

Procedure. Participants were initially asked to bring a close friend to mind and to then complete the friend questionnaire. 
For the levels-of-processing task, the trust, orderly, and imaginative lists were assigned to the semantic, other-reference, and self-reference encoding tasks respectively. The order of presentation of the 13 adjectives in the trust list presented in the semantic task was randomized. The seven orienting questions that lead to a "No" response were taken from Study 1 and 2. The six orienting questions that lead to a "Yes" response were created by replacing the "XXXX" with a synonym or near synonym of the presented trust adjective. The order of presentation of the orderly adjectives in the other-reference task was the same as in Study 2, with the three new adjectives randomly assigned to the last three trials. Likewise, the imaginative adjectives presented in the self-reference task were the same as in Study 2, with the three new adjectives randomly assigned to the last three trials. Finally, the primacy and recency trials were identical to those from Study 1, except that the semantic task was applied to the first two trials and the other-reference task was applied to the third trial.

For the recognition task, one randomized order of the items was presented. Following recognition, the imaginativeness-IAT was completed following the exact procedure used in Study 2 (i.e., the thirteen blocks in Table 3). After the IAT, participants completed the personality and behaviour self-report questionnaires (as was done in Study 1) and then the personality and behaviour other-report questionnaires. Afterward, participants were presented the debriefing form. All other aspects of the procedure are the same as in Study 1.

\section{Results}

Hypotheses were tested in the same manner as in Study 1 and 2, with the exception that the inferential tests were one-tailed because hypotheses were directional 
(nevertheless, the focus was predominately on effect size). Normality and outliers were determined using the same criteria adopted in Study 1 and 2.

Close friend. Three-quarters $(73.0 \%)$ of participants indicated that they communicate with their friend on a daily basis ( $22 \%$ weekly and $4.3 \%$ monthly), have known their friend a median of 6 years (IQR $=3$ to 10 years), know their friend extremely well $(M d n=7, I Q R=6$ to 7$)$, and are very close with their friend $(M d n=6$, $I Q R=5$ to 7$)$

Self- and other-reference effects. Three participants were excluded from analyses due to chance accuracy (i.e., .50 correct) on the semantic encoding task (two with $M=.46$ correct and one with $M=.54$ correct). One participant was excluded for using inappropriate response keys (instructions were to use the keys labeled Yes and No, but the participant also consistently used several other keys). Two participants' levels-ofprocessing data were lost due to experimenter error.

Accuracy in the semantic task was high $(M=.91 S D=.09)$. The main effect of encoding task on RTs was significant, $F(1.85288 .19)=8.97, \eta^{2}=.05, p<.001$. The semantic $(M=1,527 \mathrm{~ms}, S E=41 \mathrm{~ms})$ and self-reference $(M=1,533 \mathrm{~ms}, S E=55 \mathrm{~ms})$ tasks were executed faster than was the other-reference task $(M=1,687 \mathrm{~ms} S E=61 \mathrm{~ms})$, $M_{\text {diff }}=-160 \mathrm{~ms}, S E=49 \mathrm{~ms}, p=.001$ and $M_{\text {diff }}=-154 \mathrm{~ms}, S E=39 \mathrm{~ms}, p<.001$, respectively. The difference in mean RT between the semantic and self-reference tasks was not significant, $p>.05$. Hence, similar to Study 1 , the semantic and self-reference tasks were executed with approximately the same amount of time. The present findings showed that the other-reference task took significantly longer to complete than did the semantic and self-reference tasks. 
The two-way repeated-measures ANOVA on proportion recalled yielded a significant main effect of encoding task, $F(1.92,299.26)=213.06, \eta^{2}=.58, p<.001$, representing a strong levels-of-processing effect. The self-reference task yielded a higher proportion recalled $(M=.36, S E=.011)$ than did the semantic $(M=.13, S E=.007)$ and other-reference $(M=.29, S E=.009)$ tasks, $p \mathrm{~s}<.001$. In turn, the other-reference task yielded a higher proportion recalled than did the semantic task, $p<.001$. The main effect of recall trial was significant, $F(1,156)=14.40, \eta^{2}=.085, p<.001$, with higher recall on the second trial $(M=.27 \mathrm{SE}=.007)$ than the first trial $(M=.25 \mathrm{SE}=.006)$. Finally, the Encoding Task $x$ Recall Trial interaction was not significant, $F(1.98,308.17)=.46$, $\eta^{2}=.003, p=.63$. These data show that the self-reference effect held against an encoding condition that required participants to make judgments about a best friend, and that there was a significant other-reference effect (replicating the meta-analytic results of Symons \& Johnson, 1997)

Analysis of item gain revealed a statistically significant main effect of encoding task, $F(1.97,307.18)=3.24, \eta^{2}=.02, p=.04$. Follow-up tests showed that item gain was significantly higher following self-reference $(M=.058, S E=.005)$ and otherreference $(M=.054, S E=.005)$ than it was for semantic $(M=.041, S E=.005)$ encoding, $M_{\text {diff }}=.017, S E=.007, p=.02$ and $M_{\text {diff }}=.013, S E=.007, p=.05$, respectively. Item gain did not differ between the self- and other-reference tasks, $p=.61$. These results suggest that the self- and other-reference tasks elicited a similar amount of elaborative processing, both of which were higher than that elicited by the semantic task.

The main effect of encoding task for the analysis of item loss was not significant, $F(1.69,261.34)=1.68, \eta^{2}=.01, p=.19$. Although the proportion of item loss was lower 
following self-reference $(M=.14, S E=.02)$ and other-reference $(M=.12, S E=.02)$ than it was following semantic $(M=.17, S E=.03)$ encoding, the differences did not reach statistical significance. However, it should be noted that 33 participants $(20.2 \%)$ did not recall any semantically encoded adjectives by the end of the first recall task, which made item loss on the second recall trial impossible. When these participants were excluded from analysis, there was a significant main effect of encoding task, $F(1.64,200.59)=$ $3.89, \eta^{2}=.03, p=.03$. Follow-up comparisons showed that the proportion of item loss was lower following other-reference $(M=.13, S E=.02)$ encoding than it was following semantic $(M=.21, S E=.03)$ encoding, $M_{\text {diff }}=.08, S E=.03, p=.02$. Although selfreference $(M=.15, S E=.02)$ yielded fewer item losses than did semantic encoding, the difference reached marginal significance, $M_{\text {diff }}=.06, S E=.03, p=.08$. Nevertheless, as was expected, these results suggest that the self- and other-reference tasks elicited more relational processing than did the semantic task. Furthermore, the results suggest that the self- and other-reference tasks elicited relational processing of similar effectiveness.

Given that the self- and other-reference tasks did not differ significantly in item gain or item loss but the self-reference task clearly elicited higher recall, the estimates of the rate of approach to asymptote and asymptotic recall were compared among the three encoding tasks. The results showed that the best fitting cumulative recall curve for the semantic task had an estimated asymptote of $M=2.10(95 \%$ CI $[1.96,2.24])$ at a rate of $M=.21(95 \% \mathrm{CI}[.17, .24])$; the best fitting curve for the other-reference task had an estimated asymptote of $M=3.96(95 \% \mathrm{CI}[3.78,4.14])$ at a rate of $M=.43(95 \% \mathrm{CI}[.34$, $.52])$; and the best fitting curve for the self-reference task had an estimated asymptote of 
$M=5.12(95 \%$ CI $[4.90,5.34])$ at a rate of $M=.44(95 \%$ CI $[.36, .53]) .{ }^{24}$ Hence,

although the self- and other-reference tasks differed in asymptotic recall, the tasks did not differ in rate of approach to asymptote.

A final source of evidence to hint at what caused the higher recall in the selfreference task was an examination of the time course of recall. Figure 9 presents a plot of cumulative recall for each encoding task across the 14-minute recall period. It appeared as though the difference in cumulative recall between the self- and other-reference tasks was smallest at minute 1 , larger minutes 2 through 5 , and was approximately equal minute 6 onwards. Thus, the full recall advantage was realized by minute 6 with approximately parallel curves from then on, indicating that similar amounts of itemspecific information lead to successful retrieval of list items later in the recall period. Hence, the pattern of cumulative recall suggests that the self-reference task elicited more effective relational information than did the other-reference task (i.e., the item gains occurred fairly early in the first recall task, suggesting that the relational information was more effective at eliciting successful retrieval of list items).

\footnotetext{
${ }^{24}$ The estimates for the asymptote and the rate of approach to asymptote and their $95 \%$ confidence intervals were based on the average proportion recalled at each minute of recall, from minute 0 to 14 . Hence, these are group level estimates, as opposed to individual level estimates. I decided to fit the group level data due to the fact that many participants did not recall any semantically encoded adjectives and the estimates are directly comparable to Figure 9.
} 


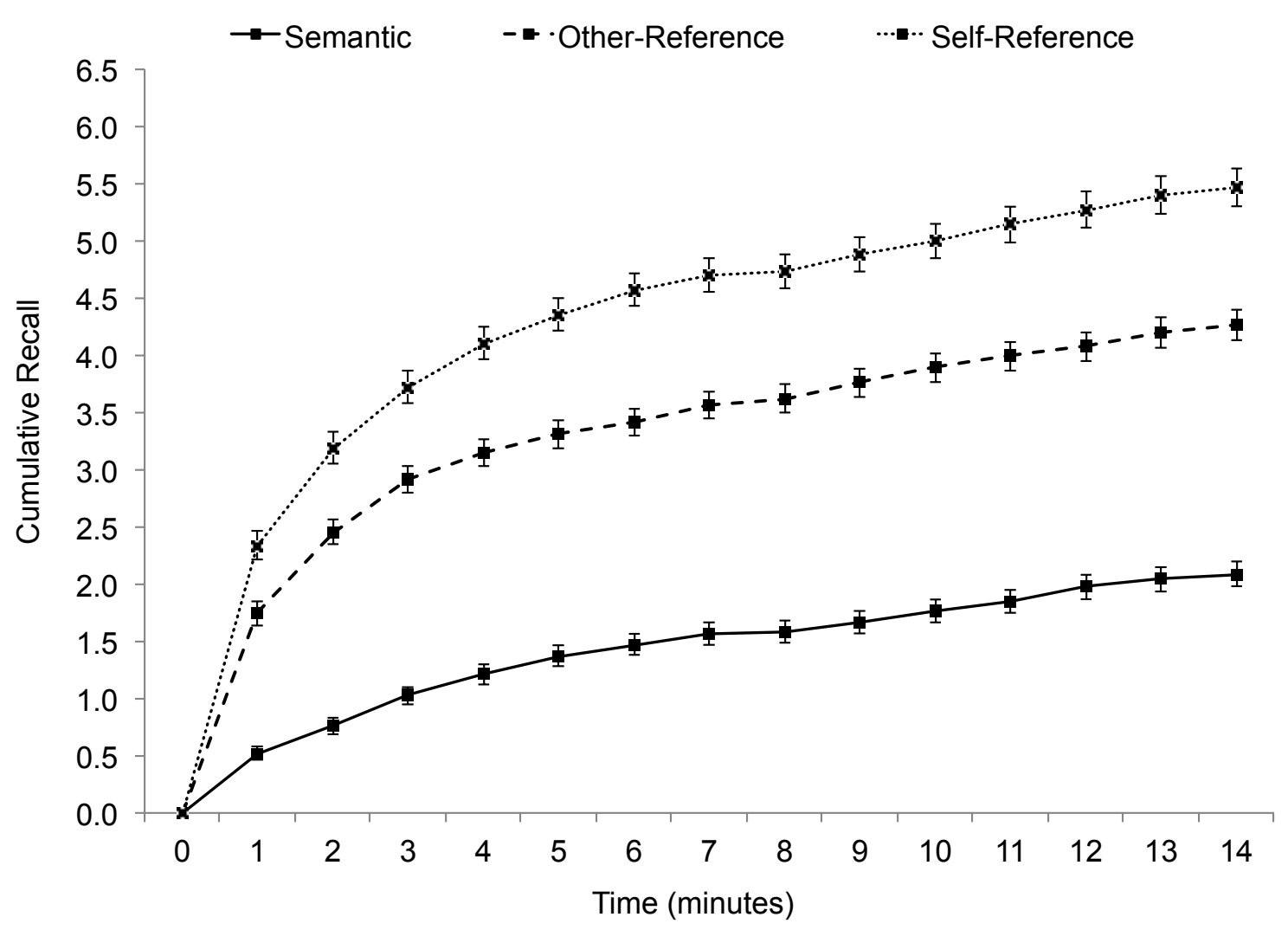

Figure 9. Cumulative recall in Study 3 as a function of encoding task. Error bars represent standard error of the mean.

Recognition test performance was analyzed in a two-way within-subjects ANOVA with encoding task (semantic, other-reference, self-reference) and item type (correct recognition, false recognition) as repeated-measures variables. Although the main effect of encoding task and item type were statistically significant, $F(1.61,256.10)$ $=35.99, \eta^{2}=.19, p<.001$ and $F(1,157)=2127.14, \eta^{2}=.93, p<.001$ respectively, particular interest was on the significant Encoding Task x Item Type interaction, $F(1.50$, $233.29)=143.24, \eta^{2}=.48, p<.001$. Simple effects analysis showed that correct recognition differed by encoding task, $F(2,156)=109.48, \eta^{2}=.58, p<.001$. Follow-up comparisons showed that trait adjectives subjected to self-reference encoding $(M=.91$, $S E=.009)$ were better recognized as having been presented in the levels-of-processing 
task than were those subjected to semantic $(M=.67, S E=.016)$ or other-reference $(M=$ $.84, S E=.011)$ encoding, $p s<.001$. Furthermore, the trait adjectives subjected to otherreference encoding were better recognized than were those subjected to semantic encoding, $p<.001$. This pattern of data mirrored the free recall results. The simple effect of encoding task on false recognition was also statistically significant, $F(2,156)=36.13$, $\eta^{2}=.32, p<.001$. Specifically, participants falsely recognized more lures of the adjectives presented in the semantic $(M=.27, S E=.02)$ and other-reference $(M=.28, S E$ $=.01)$ tasks than lures of the adjectives presented in the self-reference $(M=.19, S E=$ $.01)$ task, $p \mathrm{~s}<.001$. False recognition did not differ between the semantic and otherreference tasks, $p=.57$. These results suggest that the self-reference task elicited more item-specific processing than did the other-reference task (and the semantic task), resulting in more distinctive cues to support both correct recognition of list items and successful rejection of semantically related lures.

Lastly, there was a significant main effect of item type on proportion of yes responses in the recognition task, $F(1.93,300.50)=2814.69 \eta^{2}=.95, p<.001$. Participants responded yes to old items $(M=.81, S E=.009)$ more often than to lures $(M$ $=.24, S E=.011)$ and unrelated items $(M=.02, S E=.005), p s<.001$. In turn, participants responded yes to lures more often than to unrelated items, $p<.001$, suggesting there was significant false recognition of lures.

To summarize, the memory results confirmed a self-reference and an otherreference effect on recall and recognition (Symons \& Johnson, 1997). Item gain in the second recall trial was significantly higher for adjectives subjected to self- and otherreference encoding than it was for adjectives subjected to semantic encoding, indicating 
that differences in elaborative processing drove the self- and other-reference effects. Additionally, item loss was higher for adjectives subjected to semantic encoding than it was for adjectives subjected to self- and other-reference encoding, suggesting that a relational processing difference also drove the self- and other-reference effects. Hence, the memory results confirmed that differences in elaborative and organizational processing elicited by the self- and other-reference tasks produced the higher level of recall relative to the semantic task (Symons \& Johnson, 1997), using measures of those processes that are not subject to circularity or affected by arbitrary recall test length (Burns \& Herbert, 2006).

Although the rate of approach, item gain, and item loss results did not offer a clear explanation for why recall was higher in the self-reference task, an examination of cumulative recall suggested that the self-reference task elicited better relational processing than did the other-reference task (i.e., item gains comprising the recall advantage were early in the first recall trial). In contrast, the rate of false recognition was significantly lower for lures of the self-referenced adjectives than it was for lures of the other-referenced and semantically encoded adjectives. In addition, there was higher correct recognition for the self-referenced adjectives than the other-referenced and semantically encoded adjectives, which suggests that the processing involved in the selfreference effect rendered distinctive information (i.e., it was capable of supporting higher correct recognition and better rejection of lures). The results also suggested that the otherreference effect was driven by item-specific information that lead to a high level of correct recognition, but it did not prevent participants from false recognition, meaning the set of cues were less distinctive than were those from the self-reference task. 
Personality and self-reference. The distributions for openness, imaginativeness, encoding-openness, openness recall, imaginativeness-IAT, and false recognition were approximately normal. The estimated rate of approach to asymptote parameter was log transformed. The distributions of item gain and item loss for self-referenced adjectives were severely positively skewed, but were improved by an inverse transformation. There were five outliers in the log-transformed rate of approach distribution $(z \mathrm{~s}<-3.66)$ and three in the correct recognition distribution $(z \mathrm{~s}<-3.88)$. In the following analyses, the transformed variables were examined and outliers were only excluded when the respective variable was involved in a particular correlation.

Table 15 below displays a zero-order correlation matrix with the direct and indirect personality measures as well as the elaborative and organizational processing measures for self-referenced openness adjectives. As was expected, the direct measure of openness and imaginativeness derived from self-report ratings of dispositional phrases were significantly positively correlated. Additionally, the self-reference judgments made regarding the openness adjectives in the levels-of-processing task (i.e., encodingopenness) correlated positively with the direct measures of openness and imaginativeness, suggesting a similar source of knowledge was tapped when making personality judgments on all three measures. Further results showed that participants who were highly open to experience recalled significantly more openness adjectives by the end of the 14-minute recall period, and the same result surfaced for self-reported imaginativeness, but not for encoding-openness. These results replicated the trends noted in Study 2 and the finding in Study 1 (see Appendix K) that openness was associated with significantly higher recall of the self-referenced orderliness adjectives. In contrast, the 
estimated rate of approach to asymptote was not associated with any of the direct or indirect personality measures, thus failing to replicate the openness-recall speed relationship observed in Study 2. As was expected, higher openness recall was associated with slower recall speed. 
Table 15

Zero-Order Correlations Among Direct and Indirect Measures of Openness and Self-Reference Memory Processes in Study 3

\begin{tabular}{|c|c|c|c|c|c|c|c|c|c|c|}
\hline Measure & 1 & 2 & 3 & 4 & 5 & 6 & 7 & 8 & $9^{\mathrm{a}}$ & $10^{\mathrm{a}}$ \\
\hline 1. Openness & - & & & & & & & & & \\
\hline 2. Imaginativeness & $.59^{* * *}$ & - & & & & & & & & \\
\hline 3. Encoding-Openness & $.42^{* * *}$ & $.55^{* * *}$ & - & & & & & & & \\
\hline 4. Openness Recall & $.26^{* * *}$ & $.32^{* * *}$ & .06 & - & & & & & & \\
\hline 5. Rate of Approach & .03 & -.04 & .05 & $-.17^{*}$ & - & & & & & \\
\hline 6. Correct Recognition & $.37^{* * *}$ & $.29^{* * *}$ & $.23^{* *}$ & $.22^{* *}$ & .03 & - & & & & \\
\hline 7. False Recognition & .03 & -.05 & .03 & $-.14^{*}$ & -.01 & -.02 & - & & & \\
\hline 8. Openness-IAT & .03 & .07 & $.20^{* *}$ & -.03 & $.07^{++}$ & -.02 & -.02 & - & & \\
\hline 9. Inverse Item gain ${ }^{a}$ & -.05 & -.04 & .09 & $-.35^{* * *}$ & $.43^{* * *}$ & .02 & -.01 & .01 & - & \\
\hline 10. Inverse Item loss ${ }^{\mathrm{a}}$ & -.03 & -.01 & $-.24^{* *}$ & -.02 & $.07^{++}$ & -.01 & $-.18^{*}$ & .01 & -.01 & - \\
\hline
\end{tabular}

Note: $n=156$ to 163 . One-tailed significance tests.

${ }^{a}$ Inverse transformed. Must be interpreted accordingly.

${ }^{++} p<.20 .{ }^{+} p<.10 .^{*} p<.05 .{ }^{* *} p<.01 .{ }^{* * *} p<.00$ 
Further results presented in Table 15 showed that higher levels of correct recognition of the openness adjectives were significantly associated with higher openness, imaginativeness, and encoding-openness, whereas false recognition of semantically related lures was not associated with these direct personality measures. Given that the two recall tasks occurred before the recognition task, these data must be interpreted with caution, especially given that the direct measure of openness and imaginativeness were also related to higher recall. Specifically, the act of recalling (i.e., retrieving) more of the openness adjectives among highly open participants may have inand-of itself provided additional item-specific cues for them to use in the recognition test, thereby improving correct recognition by increasing recollection but leaving familiarity unaffected (i.e., a testing effect; see Chan \& McDermott, 2007). In support of that idea, openness in Study 1 was associated with significantly higher recall of the orderliness adjectives as well as marginally higher correct recognition (see Appendix K). Furthermore, higher levels of openness recall were associated with higher correct recognition and lower false recognition in the present study and in Study 2 (Table 8). However, although openness and imaginativeness were marginally associated with higher recall in Study 2, correct recognition and false recognition were not associated with those direct measures, which is inconsistent with the testing effect explanation offered above. Methodological limitations and inconsistencies in results aside, the recognition memory results taken at face value indicate that highly open and imaginative participants were better able to recognize the items from the self-reference task and that the processing that lead to higher levels of correct recognition did not render a set of distinctive cues, in that the cues did not also help them correctly reject semantically-related lures. 
The last three rows of Table 15 show that the openness-IAT was positively correlated with encoding-openness, however it did not relate to the other direct measures or the memory-based ones, again failing to replicate the trends observed in Study 1. Item gain was not associated with any of the direct or indirect measures. As was expected, item gain was positively associated with openness recall and negatively associated with recall speed. Item loss was significantly associated with false recognition in the positive direction, indicating that participants who lost more items in the second recall task also falsely recognized more lures in the recognition test, which is inconsistent with the expectation that they both measure organizational processing (i.e., higher degrees of organizational processing were expected to lead to higher false recognition and fewer item losses). Item loss was also significantly associated with encoding-openness in the positive direction. Specifically, participants who were high on the encoding-openness measure lost more items in the second recall trial (i.e., they engaged in less relational processing). Lastly, as was expected, higher item gain was associated with slower recall speed.

To summarize, the negative openness-recall speed relationship observed in Study 2 did not replicate. In contrast, the trends noted in Study 2 that highly open and imaginative participants recalled more openness adjectives were stronger and statistically significant. Higher directly measured openness, imaginativeness, and encoding-openness were also associated with higher correct recognition. However, whether these relationships were due to a testing effect or a genuine difference in the encoding of itemspecific information was unclear. 
Personality and cumulative recall. Figure 10 below presents a plot of cumulative recall for participants who were low $(\mathrm{n}=54)$, medium $(\mathrm{n}=56)$, or high $(\mathrm{n}=$ 48) on the direct measure of openness. Unlike what was observed in Study 2, the rate of approach to asymptote was similar among the openness groups (i.e., the three lines in the top of Figure 10 increase at similar rates over time). Clearly, however, the openness groups differed in amount recalled across the entire recall period, with higher levels of openness being associated with higher recall. Although there did not appear to be differences in item-specific processing (e.g., the slope of the lines is similar and the lines do not cross at any point in time), the pattern of cumulative recall suggests that participants who were higher in openness engaged in more organizational processing, allowing for a more effective search of memory for item-specific cues, eliciting higher recall early in the first recall task. For example, at minute 1, the high openness group had nominally higher cumulative recall than did the medium openness group, and the medium group had nominally higher cumulative recall than did the low openness group. Although the differences in cumulative recall among the groups increased somewhat over the recall period, the differences did not elicit significant item gain or slower recall according to the rate of approach parameter. Hence, it seems most likely that the difference in recall was due to more effective relational processing among open participants. Unfortunately, the item loss measure did not corroborate this explanation. That is, if open participants engaged in more effective relational processing, then the item loss measure would have been negatively associated with openness (which was not the case; see Table 15). It is important to note that these results are similar to the time course of recall of the orderliness adjectives that were self-referenced as a function of openness in Study 1 (see 
Figure 6) and to the pattern observed in Study 2 (see Figure 5), with the exception of the high openness groups' slow recall (which was predicted).

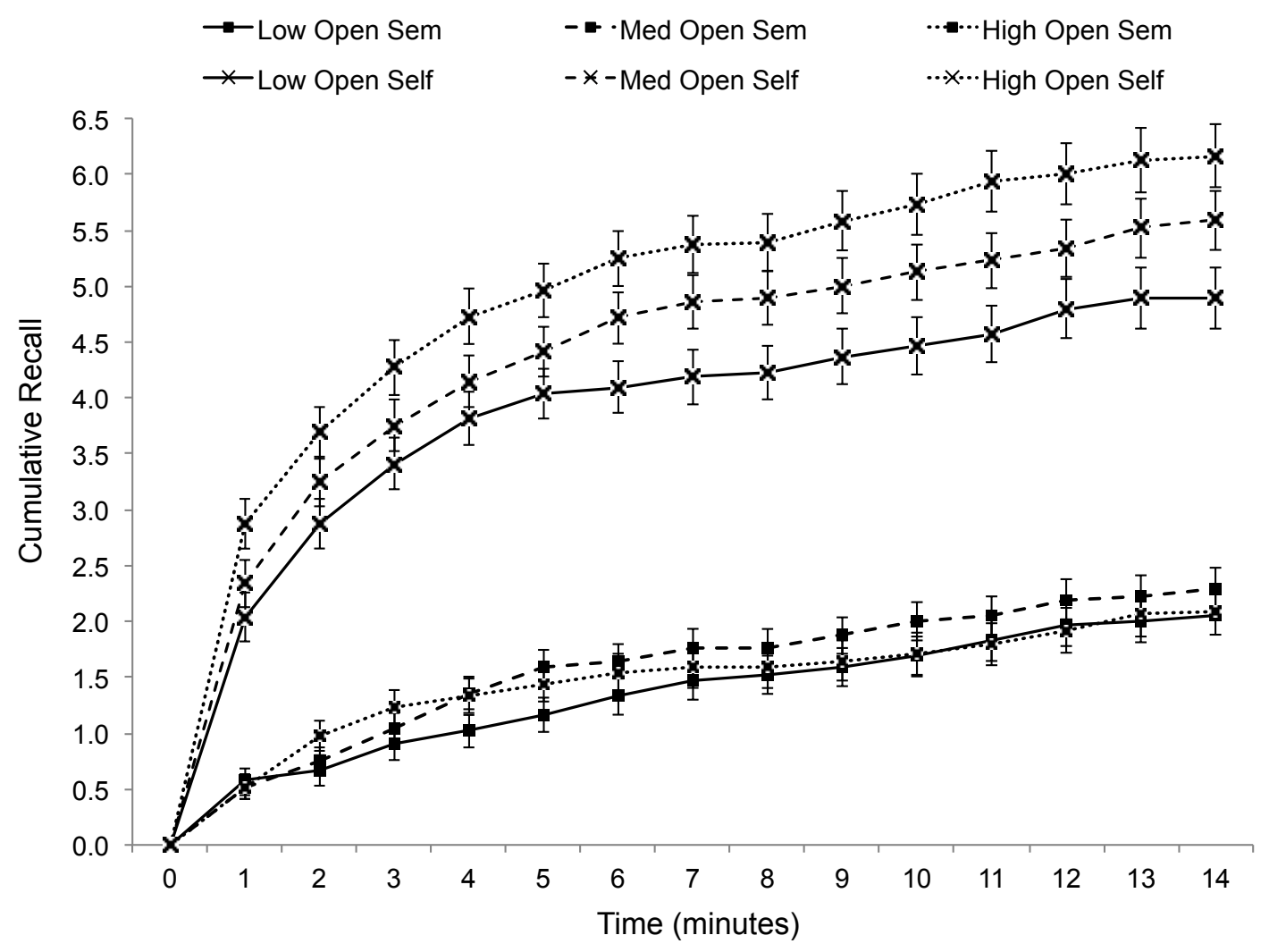

Figure 10. Cumulative recall in Study 3 as a function of openness presented separately for the self-reference and semantic tasks. Error bars represent standard error of the mean. Open $=$ Openness. Sem $=$ Semantic. Self $=$ Self-reference.

Divergent Validity. To test the hypotheses concerning divergent validity, correlations were examined between the self-reported personality and memory-based measures from the self-reference task and participants' personality self-reports of conscientiousness and orderliness as well as their reports of their friend's conscientiousness, orderliness, openness, and imaginativeness. While it was hypothesized that there would be a significant negative relationship between participants self-reported openness and recall speed of self-referenced openness adjectives (which did not occur, as 
was discussed in the previous section), it was also expected that the relationship between recall speed and other personality measures (self and other-reports) would not be statistically significant (i.e., supporting divergent validity). Particular attention was paid to personality measures that explained variation in recall speed and item gain in the second recall task.

The results presented in Table 16 showed that participants who rated their friend as particularly orderly recalled the openness adjectives significantly more quickly than did those who viewed their friend as less orderly. It is possible that participants who viewed their friend as highly orderly recalled the self-referenced openness adjectives more quickly because there was relatively more activation of item-specific information from the other-reference task, eliciting slower recall of those adjectives and artificially speeding-up recall of the openness adjectives. These data hint that the failure to replicate the negative openness-recall speed relationship observed in Study 2 was due to the fact that participants had to make judgments about their own personality and their best friend's personality within the same task (i.e., due to the within-subjects design). The remaining results presented in Table 16 showed that none of the direct personality measures were significantly negatively related to rate of approach or positively related to item gain. 
Table 16

Zero-Order Correlations Among Conscientiousness and Orderliness and Self-Reference Measures of Openness

\begin{tabular}{ccccccc}
\hline $\begin{array}{c}\text { Memory-based } \\
\text { Measures }\end{array}$ & Self & Self & Friend & Friend & Friend & Friend \\
\hline Openness Recall & $-.07^{++}$ & $-.14^{*}$ & $-.08^{++}$ & $-.10^{+}$ & -.03 & .05 \\
Rate of Approach & .05 & .04 & $.08^{++}$ & $.16^{*}$ & $.13^{+}$ & .08 \\
Inverse Item gain ${ }^{\mathrm{a}}$ & -.05 & -.02 & $.09^{++}$ & $.11^{+}$ & $.16^{*}$ & $.09^{++}$ \\
Inverse Item loss $^{\mathrm{a}}$ & .00 & $.10^{++}$ & .06 & .05 & $.08^{++}$ & $.07^{++}$ \\
\hline
\end{tabular}

Note: $n=158$ to 163 .

${ }^{\text {a }}$ Inverse transformed. Must be interpreted accordingly. ${ }^{++} p<.20{ }^{+} p<.10 .^{*} p<.05 .{ }^{* *} p<.01 .{ }^{* * *} p<.001$.

To explore whether the negative relationship between self-reported openness and recall speed of the openness adjectives was affected by participants' rating of their best friend's orderliness, the correlations presented in Table 15 were broken down by whether participants rated their friend as low $(n=55)$, medium $(n=51)$, or high $(n=52)$ on the direct measure of orderliness. Results presented in Table 17 showed that the relationship between the direct measures of openness and imaginativeness and the rate of approach tended to differ depending on participants' rating of their best friend's orderliness. In particular, at a low level of orderliness, the direction of the relationship for openness $(r=$ $.16)$ and imaginativeness ( $r=.21)$ was positive; at a medium level, it was negative for openness $(r=-.14)$ and close to zero for imaginativeness $(r=.03$; which is similar to the pattern observed in Study 2); but at a high level, it was close to zero for openness ( $r=$ 
$.02)$ and negative for imaginativeness $(r=-.21)$. These results suggest that the level of participants' best friend's orderliness (which was hypothesized to slow down recall of the other-referenced adjectives) may have interfered with recall speed of the openness adjectives. To test this idea formally, the rate of approach parameter for the selfreferenced openness adjectives was analyzed in a one-way analysis of covariance (ANCOVA), with other-reported orderliness (low, medium, high) as a between-subjects variable and self-reported openness as a continuous covariate. The interaction between other-reported orderliness and self-reported openness was marginally significant, $F(2$, $157)=2.75, \eta^{2}=.03, p=.07$, supporting the observation that the association between self-reported openness and recall speed tended to differ as a function of the participants' rating of their best friend's orderliness. In sum, the data hint that the failure to replicate may be due to the within-subjects design that required participants to tap into their selfconcept and their concept of one of their best friends within the same memory task.

Table 17

Zero-Order Correlations Among the Direct and Self-Reference Measures of Openness as a Function of Friend's Level of Orderliness

\begin{tabular}{cccccccc}
\hline Friend's & Self-Report and & & & & & & \\
Orderliness & Memory-based & 1 & 2 & 3 & 4 & 5 & 6 \\
& Measures & & & & &
\end{tabular}

\begin{tabular}{|c|c|c|c|c|}
\hline Low & 1. Openness & - & & \\
\hline & 2. Imaginativeness & $.57^{* * *}$ & - & \\
\hline & 3. Encoding-Openness & $.44^{* * *}$ & $.53^{* * *}$ & - \\
\hline & 4. Openness Recall & $.20^{+}$ & $.37^{* *}$ & .01 \\
\hline
\end{tabular}


5. Rate of Approach $\quad .16^{++} \quad .21^{+} \quad .34^{* *} \quad-.03 \quad$ -

$\begin{array}{lllllll}\text { 6. Inverse Item gain }{ }^{\mathrm{a}} & .07 & .01 & .26^{*} & -.38^{* *} & .53^{* * * *} & - \\ \text { 7. Inverse Item loss }{ }^{\mathrm{a}} & -.06 & .01 & -.32^{* *} & .28^{*} & -.24^{*} & -.19^{+}\end{array}$

Medium 1. Openness

2. Imaginativeness

$.62^{* * *}-$

3. Encoding-Openness $.41^{* *} \quad .48^{* * *} \quad-$

4. Openness Recall $\quad .45^{* * *} \quad .32^{*} \quad .15^{++} \quad$ -

5. Rate of Approach $\quad \begin{array}{llllll}.14 & .03 & .17^{++} & -.15^{++} & -\end{array}$

6. Inverse Item gain ${ }^{\mathrm{a}} \quad \begin{array}{lllllll}-.06 & .01 & .06 & -.36^{* *} & .29^{*} & -\end{array}$

7. Inverse Item loss ${ }^{\text {a }} \quad \begin{array}{lllllll}-.12 & .08 & -.15^{++} & -.43^{* * *} & .15^{++} & .17^{++}\end{array}$

High $\quad 1$. Openness

2. Imaginativeness $\quad .69^{* * *} \quad-$

3. Encoding-Openness $.52^{* * *} .72^{* * *} \quad-$

4. Openness Recall $\quad .20^{+} \quad .19^{+} \quad .08 \quad$ -

5. Rate of Approach $\quad \begin{array}{lllll}.02 & -.21^{+} & -.18^{++} & .29^{*} & -\end{array}$

6. Inverse Item gain ${ }^{\mathrm{a}} \quad-.17^{++} \quad-.22^{+} \quad .00 \quad-.50^{* * *} \quad .47^{* * *} \quad-$

7. Inverse Item loss ${ }^{\mathrm{a}} \quad \begin{array}{lllllll} & -.04 & -.11 & -.14^{++} & .11 & .20^{+} & -.03\end{array}$

Note: $n=51$ to 55 . One-tailed tests.

${ }^{a}$ Inverse transformed. Must be interpreted accordingly.

${ }^{++} p<.20 .{ }^{+} p<.10 .{ }^{*} p<.05 .{ }^{* *} p<.01 .{ }^{* * *} p<.001$.

It is noteworthy that the pattern of interrelationships in Table 17 among the openness measure and rate of approach to asymptote was similar to that observed in Study 2, but only at the medium level of participants' friend's orderliness. That is, the 
direct measure of openness was negatively associated with the rate of approach parameter ( $r=-.14$; although the magnitude was smaller), but item gain was not. In order to compare the time course of recall to that of Study 2 at the medium level of participants' best friend's orderliness in Study 3, Figure 11 plots cumulative recall for the selfreferenced adjectives separately for those who were low $(n=17)$, medium $(n=22)$, or high $(n=12)$ on the direct measure of openness. Interestingly, the qualitative pattern of cumulative recall appears similar to that observed in Study 2 (see Figure 5). Specifically, the low openness group had the nominally lowest cumulative recall at minute 2 onwards, with minimal item gain after minute 5 . The medium and high openness groups had a similar time course of recall, with the important exception that the high openness group tended to have a lower level of cumulative recall early in the first recall task (i.e., minutes 2 and 3 ) but a higher level of cumulative recall later in the recall period (i.e., minutes 6 onwards), suggesting that the high openness group processed more item-specific information (which slowed down recall). The fact that the pattern of correlations and cumulative recall are similar to that observed in Study 2, but only at the medium level of participants' best friend's orderliness, supports the idea that the time course of recall for the self-reference task was affected by the participants' perception of their best friend's orderliness. The pattern may only surface at the medium level because the trait does not differentiate their friend from most other people (e.g., it is not what stands out about them). 


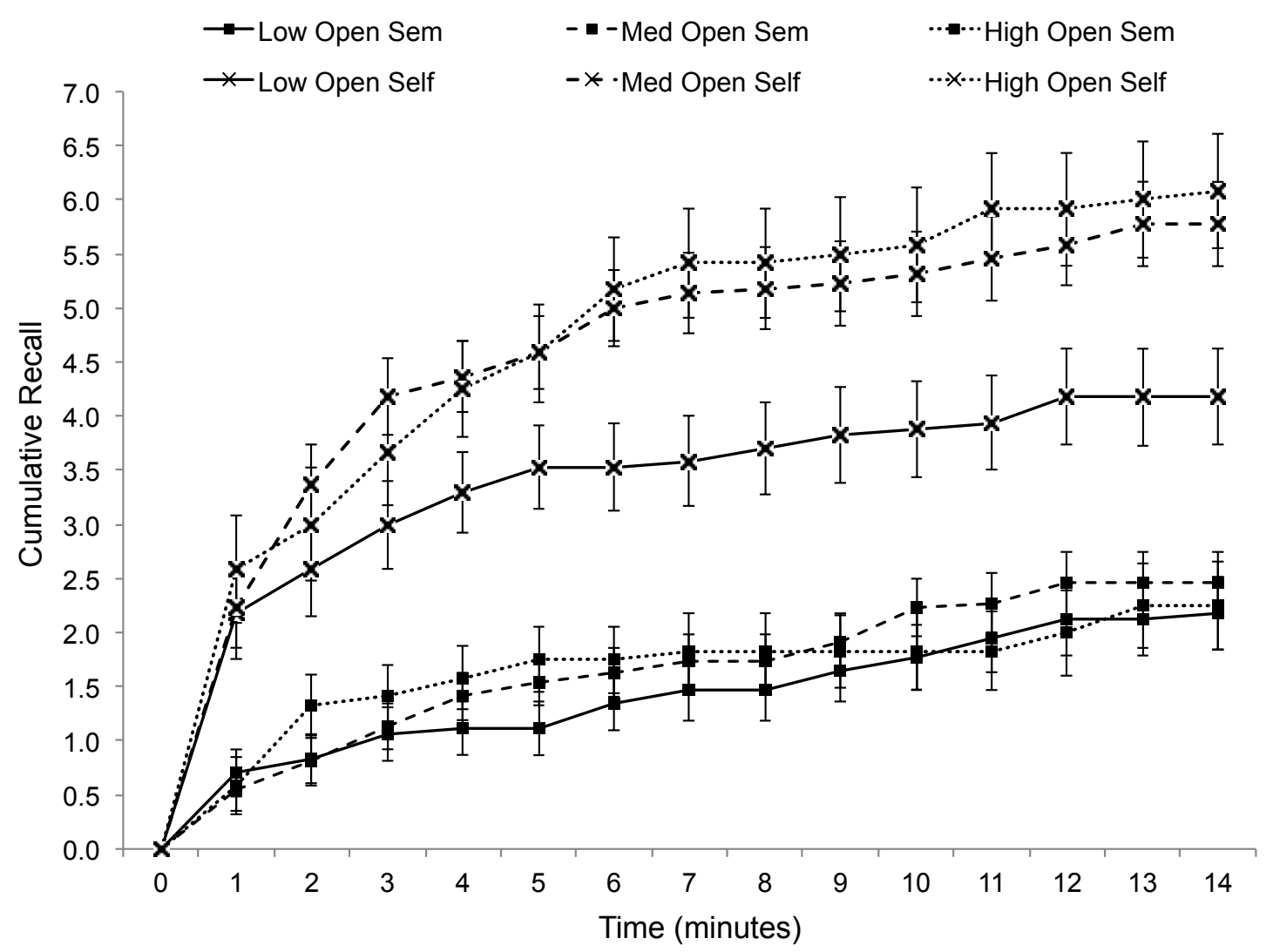

Figure 11. Cumulative recall in Study 3 as a function of openness at the medium level of best friend's orderliness presented separately for the self-reference and semantic tasks. Error bars represent standard error of the mean. Open $=$ Openness. Sem $=$ Semantic. Self $=$ Self-reference.

Self-reported and observed behaviour. One-tailed correlations and independent samples t-tests were computed to address the hypotheses concerning predictive validity of the memory-based measures of personality self-concept. The self-report items examined in Study 2 were included in the analysis. Whether participants decided to take the debriefing sheet was examined as the sole measure of observed openness behaviour. The continuous self-report measures of openness attitudes were examined for normality and outliers. All distributions were approximately normal. In the following analyses, outliers and missing data identified earlier were excluded. Table 18 presents the zero- 
order correlations among the direct, indirect, and memory-based measures of personality and the continuous self-report measures of openness attitudes.

Table 18

Zero-Order Correlations Between Direct, Indirect, and Self-Reference Memory Measures and Self-Reported Openness Attitudes

\begin{tabular}{|c|c|c|c|}
\hline Personality Measure & $\begin{array}{c}\text { General } \\
\text { Intelligence }\end{array}$ & $\begin{array}{c}\text { Romantic } \\
\text { Partner }\end{array}$ & $\begin{array}{c}\text { Voting } \\
\text { Conservative }\end{array}$ \\
\hline 1. Openness & $.17^{*}$ & $.21^{* *}$ & $-.44^{* * *}$ \\
\hline 2. Imaginative & $.15^{*}$ & $.24^{* * *}$ & $-.22^{* *}$ \\
\hline 3. Encoding-Openness & $.28^{* * *}$ & $.15^{*}$ & -.08 \\
\hline 4. Openness Recall & $.22^{* *}$ & $.12^{+}$ & $-.19^{* *}$ \\
\hline 5. Rate of Approach & $-.12^{+}$ & .01 & .00 \\
\hline 6. Correct Recognition & .06 & -.02 & -.06 \\
\hline 7. False Recognition & -.09 & -.07 & -.03 \\
\hline 8. Openness-IAT & $.10^{+}$ & .02 & -.04 \\
\hline 9. Inverse Item gain ${ }^{a}$ & $-.17^{*}$ & -.05 & .01 \\
\hline 10. Inverse Item loss ${ }^{a}$ & .02 & $.14^{*}$ & $-.12^{+}$ \\
\hline
\end{tabular}

Note. $n=159$ to 163 . One-tailed tests.

${ }^{a}$ Inverse transformed. Must be interpreted accordingly.

${ }^{+} p<.10 .^{*} p<.05 .^{* *} p<.01 .^{* * *} p<.001$.

The direct measures of openness, imaginativeness, and encoding-openness were significantly associated with self-reported general intelligence. Specifically, as has been found in previous research, participants who were highly open, imaginative, and endorsed more openness adjectives as self-descriptive reported higher levels of general intelligence relative to their peers. Further, participants who reported higher levels of general intelligence also recalled significantly more openness adjectives, gained significantly 
more openness adjectives in the second recall task, and had marginally slower recall speed and stronger openness-IAT effects. Further results showed that participants who indicated a preference for a romantic partner who is open to experience reported higher levels of openness, imaginativeness, and encoding-openness, and recalled marginally more openness adjectives and had significantly fewer item losses. Directly measured openness and imaginativeness, as well as openness recall, were significantly negatively related to the probability of voting for the conservative party, while more item losses were marginally related to a higher probability of voting for the conservative party. All the associations noted above were in the predicted direction.

Table 19 presents the means and standard deviations of the direct, indirect, and memory-based measures of openness as a function of the binary self-reported openness attitudes and behaviours. Results show that participants who decided to take the debriefing sheet at the end of the experimental session had a significantly stronger imaginativeness-IAT effect, $M_{\mathrm{diff}}=.10, t(152)=1.96, p=.026$, but lower self-reported imaginativeness, $M_{\text {diff }}=.33, t(153)=-2.18, p=.031$. Participants who indicated that they enjoy reading classic literature were more highly open to experience $\left(M_{\mathrm{diff}}=.65\right.$, $t(155.14)=6.98 p<.001)$, imaginative $\left(M_{\text {diff }}=.56, t(161)=5.67 p<.001\right)$, endorsed more openness adjectives as self-descriptive $\left(M_{\text {diff }}=.11, t(159)=3.88 p<.001\right)$, recalled more openness adjectives $\left(M_{\text {diff }}=.57, t(161)=1.74, p=.04\right)$, and correctly recognized more openness adjectives $\left(M_{\text {diff }}=.04, t(156.91)=3.26, p<.001\right)$. Participants who reported that they play a musical instrument were significantly more open to experience $\left(M_{\mathrm{diff}}=.24, t(161)=2.14, p=.02\right)$, marginally more imaginative $\left(M_{\mathrm{diff}}=.16, t(161)=\right.$ $1.63, p=.07)$, and had a significantly strongly imaginativeness-IAT effect $\left(M_{\text {diff }}=.07\right.$, 
$t(160)=1.97, p=.03)$. Lastly, participants who indicated that their program of study was in the liberal arts recalled the openness adjectives more quickly $\left(M_{\text {diff }}=.13, t(156)=\right.$ $1.84, p=.03)$, falsely recognized fewer openness adjectives $\left(M_{\text {diff }}=-.06, t(160)=-2.86\right.$, $p<.01)$, and correctly recognized marginally more openness adjectives $\left(M_{\text {diff }}=.02\right.$, $t(157)=1.26, p=.10)$. Overall, as has been observed thus far, the predictive validity of the memory-based measures was fairly poor. 
Table 19

Means and Standard Deviations of the Direct, Indirect, and Self-Reference Memory Measures as a Function of Self-Reported Openness Attitudes and Behaviours

\begin{tabular}{lcccccccc}
\hline & \multicolumn{2}{c}{ Debriefing Sheet } & \multicolumn{2}{c}{ Classic Literature } & \multicolumn{2}{c}{ Musical Instrument } & Liberal Arts Degree \\
\hline Personality Measure & No & Yes & No & Yes & No & Yes & No \\
$(\mathrm{n}=23)$ & $(\mathrm{n}=132)$ & $(\mathrm{n}=94)$ & $(\mathrm{n}=64)$ & $(\mathrm{n}=105)$ & $(\mathrm{n}=53)$ & $(\mathrm{n}=69)$ & $(\mathrm{n}=89)$ \\
\hline 1. Openness & $3.74^{++}(.75)$ & $3.58(.67)$ & $3.36(.65)$ & $4.00^{* * *}(.52)$ & $3.54(.67)$ & $3.77^{*}(.68)$ & $3.61(.65)$ & $3.62(.71)$ \\
2. Imaginativeness & $3.96^{*}(.72)$ & $3.63(.66)$ & $3.45(.65)$ & $4.02^{* * *}(.59)$ & $3.62(.65)$ & $3.79^{+}(.72)$ & $3.64(.69)$ & $3.71(.67)$ \\
3. Encoding-Openness & $.62(.19)$ & $.66^{++}(.18)$ & $.62(.18)$ & $.73^{* * *}(.16)$ & $.65(.18)$ & $.69^{+}(.17)$ & $.65(.16)$ & $.67^{++}(.19)$ \\
4. Openness Recall & $6.09^{++}(2.78)$ & $5.33(1.92)$ & $5.21(2.01)$ & $5.78^{*}(2.02)$ & $5.44(2.09)$ & $5.44(2.02)$ & $5.54(1.96)$ & $5.37(2.15)$ \\
5. Rate of Approach & $-.26(.40)$ & $-.30(.45)$ & $-.25(.44)$ & $-.33(.42)$ & $-.27(.44)$ & $-.31(.43)$ & $-.36(.43)$ & $-.23^{+}(.43)$ \\
6. Correct Recognition & $.92(.09)$ & $.91(.09)$ & $.90(.10)$ & $.94^{* *}(.07)$ & $.91(.09)$ & $.93(.09)$ & $.91(.10)$ & $.93(.08)$ \\
7. False Recognition & $.21^{++}(.17)$ & $.18(.14)$ & $.18(.14)$ & $.19(.15)$ & $.20^{++}(.15)$ & $.17(.13)$ & $.22^{* *}(.15)$ & $.16(.14)$ \\
8. Openness-IAT & $.36(.22)$ & $.46^{*}(.23)$ & $.44(.20)$ & $.46(.26)$ & $.42(.22)$ & $.50^{*}(.24)$ & $.42(.22)$ & $.47^{++}(.23)$ \\
9. Item gain ${ }^{\text {a }}$ & $.95(.05)$ & $.95(.06)$ & $.95(.06)$ & $.95(.06)$ & $.95(.06)$ & $.95(.06)$ & $.94(.06)$ & $.95^{++}(.06)$ \\
10. Item loss ${ }^{\text {a }}$ & $.88(.15)$ & $.90(.13)$ & $.91^{++}(.13)$ & $.88(.13)$ & $.91^{++}(.12)$ & $.88(.15)$ & $.89(.14)$ & $.91(.12)$ \\
\hline
\end{tabular}

Note. $n=155$ to 163 . One-tailed significance tests.

${ }^{a}$ Inverse transformed. Must be interpreted accordingly.

${ }^{++} p<.20 .{ }^{+} p<.10 .{ }^{*} p<.05 .{ }^{* *} p<.01$. 
Personality and other-reference. The distributions for conscientiousness, orderliness, encoding-orderliness, and orderliness recall were approximately normal. The distribution for the estimated rate of approach to asymptote was log transformed. The item gain and loss measures for other-referenced adjectives were inverse transformed. There were six outliers for the rate of approach to asymptote $(z \mathrm{~S}<-3.48)$, one outlier for correct recognition $(z=-5.22)$, and one outlier for false recognition $(z=3.97)$. In the following analyses, the transformed variables were examined and outliers were only excluded when the respective variable was involved in a particular correlation.

Table 20 below displays a zero-order correlation matrix with the direct and indirect personality measures as well as the elaborative and organizational processing measures for other-referenced orderliness adjectives. As one would expect, the direct measure of conscientiousness and orderliness derived from other-report ratings of dispositional phrases were significantly positively correlated. Additionally, the otherreference judgments made regarding the orderliness adjectives (i.e., encodingorderliness) correlated positively with the direct measures of conscientiousness and orderliness, suggesting a similar source of knowledge was tapped when making personality judgments on all three measures. 
Table 20

Zero-Order Correlations Among Direct Measures of Orderliness and Other-Reference Memory Processes in Study 3

\begin{tabular}{|c|c|c|c|c|c|c|c|c|c|}
\hline Measure & 1 & 2 & 3 & 4 & 5 & 6 & 7 & $8^{\mathrm{a}}$ & $9^{\mathrm{a}}$ \\
\hline 1. Conscientiousness & - & & & & & & & & \\
\hline 2. Orderliness & $.73^{* * *}$ & - & & & & & & & \\
\hline 3. Encoding-Orderliness & $.61^{* * *}$ & $.51^{* * *}$ & - & & & & & & \\
\hline 4. Orderliness Recall & -.06 & -.08 & -.02 & - & & & & & \\
\hline 5. Rate of Approach & $-.15^{*}$ & $-.14^{*}$ & $-.12^{+}$ & $-.22^{* *}$ & - & & & & \\
\hline 6. Correct Recognition & -.01 & .02 & $.12^{+}$ & $.40^{* * *}$ & -.01 & - & & & \\
\hline 7. False Recognition & .07 & $.11^{+}$ & $.11^{+}$ & $-.13^{*}$ & $-.22^{* *}$ & $.11^{+}$ & - & & \\
\hline 8. Inverse Item gain & -.03 & .01 & $-.11^{+}$ & $-.44^{* * *}$ & $.49^{* * *}$ & $-.15^{*}$ & .00 & - & \\
\hline 9. Inverse Item loss & .01 & -.01 & $-.12^{+}$ & -.03 & $-.10^{++}$ & $.14^{*}$ & .06 & -.03 & - \\
\hline
\end{tabular}

Note: One-tailed significance tests. $\mathrm{n}=154$ to 162 .

a Inverse transformed. Must be interpreted accordingly.

${ }^{++} p<.20 .{ }^{+} p<.10 .{ }^{*} p<.05 .{ }^{* *} p<.01 .{ }^{* * * *} p<.00$ 
Participants who rated their best friend as highly conscientious, orderly, or who endorsed more orderliness adjectives as descriptive of their friend did not recall more orderliness adjectives by the end of the 14-minute recall period. As was hypothesized, participants who viewed their best friend as highly conscientious or orderly exhibited significantly slower recall speed, with encoding-orderliness being marginally associated with slower recall speed (a similar magnitude as was observed in Study 1). Hence, the main finding of Study 1 was successfully extended in Study 3 to the other-reference task, suggesting that making personality judgments about a best friend who is high on the trait reflected by the memory list materials rendered more elaborative processing of the items during encoding, which slowed down successful retrieval of list items. As has been typically observed in the present studies, higher recall was associated with slower recall speed.

Personality and cumulative recall. Figure 12 below plots cumulative recall for participants who judged their best friend's orderliness as low $(n=54)$, medium $(n=52)$, or high $(n=51)$. A close examination of cumulative recall suggests that recall speed was slower among those who judged their friend as being highly orderly, although the differences in recall speed appeared to be more subtle (and the magnitude of the relationship was smaller than that observed in Study 1). In particular, the low orderliness group recalled the other-referenced orderliness adjectives more quickly than did the medium and high orderliness groups early in the recall period and consistently across the first 12 minutes of recall (the largest differences were in the 3 to 7 minute range), whereas the high orderliness group recalled as many adjectives as the low orderliness group by the end of the recall period (their cumulative recall overlaps from minutes 12 to 
14). This pattern is qualitatively similar to that observed in Study 1 (see Figure 3), although the pattern is less pronounced, and cumulative recall for the high orderliness group was not clearly lower than the low and medium orderliness groups early in the first recall trial. Given that participants' direct measures of their friends' conscientiousness and orderliness were not associated with item loss (see Table 20), the results favour the elaborative processing explanation for the differences in recall speed. It is notable that item gain was not associated with the direct measures of personality either (except a marginal negative association with encoding-orderliness) perhaps because the personality differences in cumulative recall were slightly larger in the first recall task (i.e., up to minute 7) and that the estimated rate of approach to asymptote is a more sensitive measure of elaborative processing than is item gain. The consistency in the pattern of the correlations among the direct personality measures, the rate of approach, item gain, and item loss between Study 1 and Study 3 suggests that the representations comprising the self and the representations comprising a close other are both capable of eliciting more elaborative processing when the memory list adjectives are seen as highly descriptive of the self or the close other. ${ }^{25}$

\footnotetext{
${ }^{25}$ Group level estimates for the best fitting cumulative recall curve (Equation 1) were calculated for the orderliness groups' other-reference task performance in Study 3 as presented in Figure 12. The low orderliness group's asymptote was $\mathrm{n}(\alpha)=4.33(95 \%$ CI $[3.96,4.70])$ at a rate of $\lambda=.27(95 \%$ CI [. 19 , $.35])$; the medium orderliness group's asymptote was $\mathrm{n}(\alpha)=4.19(95 \% \mathrm{CI}[3.80,4.57])$ at a rate of $\lambda=.25$ $(95 \%$ CI $[.18, .33])$; and the high orderliness group's asymptote was $\mathrm{n}(\alpha)=4.36(95 \% \mathrm{CI}[3.96,4.77])$ at a rate of $\lambda=.24(95 \% \mathrm{CI}[.17, .30])$. These fits suggest that the rate of approach for the orderliness groups did not differ. The continuous individual difference measure of orderliness likely provided a more powerful hypothesis test of association with recall speed. However, the results here echo that the orderliness-recall speed association was indeed small.
} 


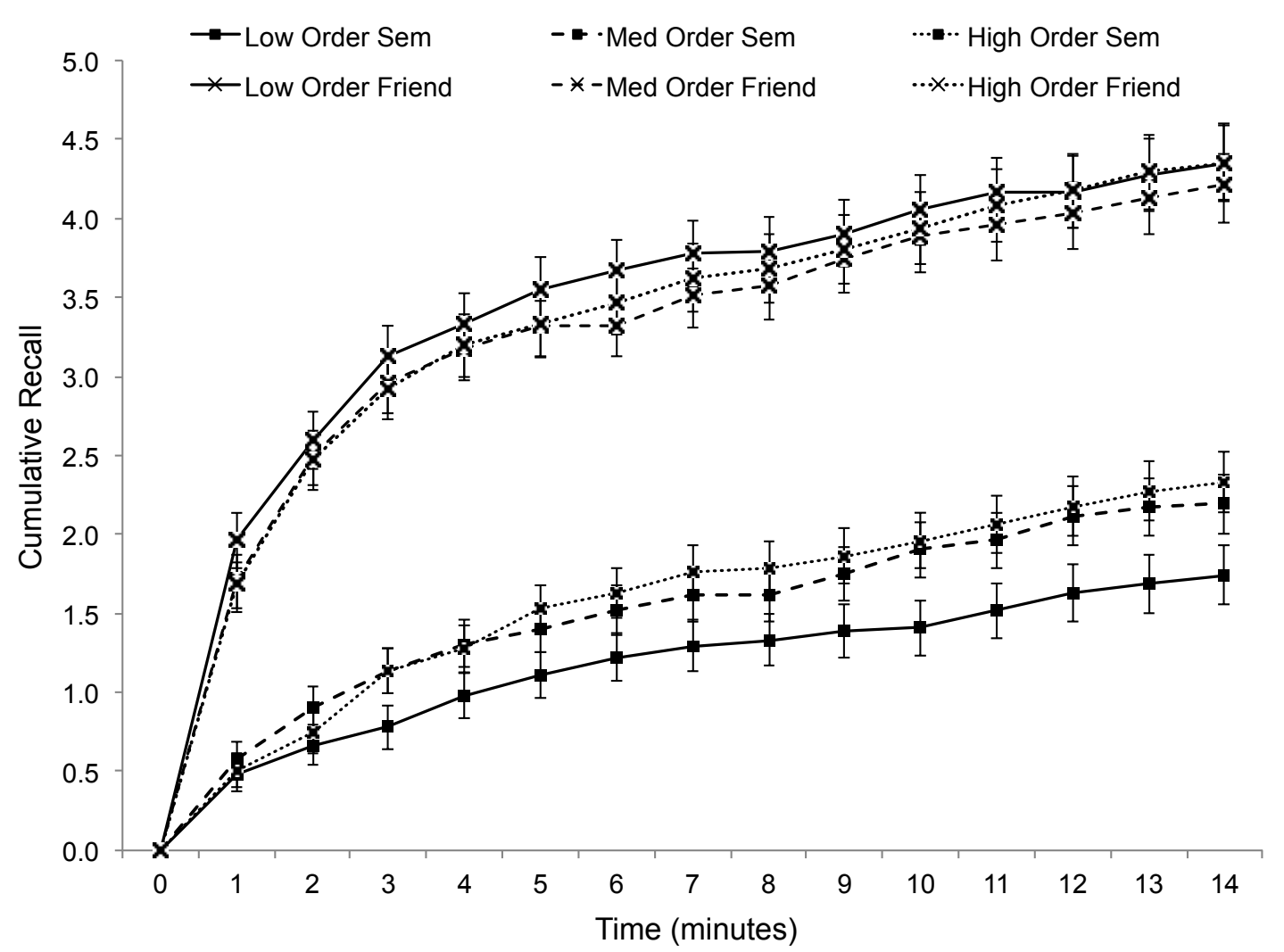

Figure 12. Cumulative recall in Study 3 as a function of orderliness presented separately for the other-reference and semantic task. Error bars represent standard error of the mean. Order $=$ Orderliness. Sem $=$ Semantic. Friend $=$ Other-reference.

Divergent Validity. It was hypothesized that there would be a significant negative relationship between participants' best friend's orderliness and recall speed of other-referenced orderliness adjectives (which did occur, as discussed in the previous section) and that the relationships between other personality measures (self- and otherreports) and recall speed would not be significant. To test the hypotheses concerning divergent validity, correlations were examined between the memory-based measures from the other-reference task and the participants' ratings of their best friend's openness and imaginativeness as well as reports of their own conscientiousness, orderliness, openness, and imaginativeness. Particular attention was paid to personality measures that 
explain variation in recall speed of the orderliness adjectives and item gain in the second recall task.

The results presented in Table 21 showed that participants who rated themselves as highly open tended to recall the orderliness adjectives more slowly, they gained significantly more orderliness adjectives in the second recall task, and they recalled significantly more orderliness adjectives than did participants who rated themselves as less open. These results do not seem to support the divergent validity of the rate of approach measure, in that these associations should have only been evidenced in relation to participants' ratings of their best friend's orderliness (i.e., in Table 20). However, as was observed with the self-reference data, it is possible that these associations were a product of the within-subjects design, wherein participants brought to mind a best friend's personality as well as their own. The remaining results presented in Table 21 show that none of the personality measures were significantly negatively related to the rate of approach parameter or positively related to item gain.

Table 21

Zero-Order Correlations Among Participants' Best Friend's Openness and Imaginativeness and Direct, Indirect, and Other-Reference Measures of Orderliness

\begin{tabular}{ccccccc}
\hline $\begin{array}{c}\text { Memory-Based } \\
\text { Measures }\end{array}$ & Friend's & Friend's & Self & Self & Self & Self- \\
& Open & Imagine & Consc. & Order & Open & Imagine \\
\hline 1. Orderliness Recall & .02 & .03 & -.07 & -.02 & $.16^{*}$ & $.13^{*}$ \\
2. Rate of Approach & -.00 & $-.09^{++}$ & -.03 & $.07^{++}$ & $-.10^{++}$ & .06 \\
3. Inverse Item gain ${ }^{\text {a }}$ & -.04 & -.04 & -.04 & -.03 & $-.16^{*}$ & -.08 \\
4. Inverse Item loss $^{\text {a }}$ & .00 & $-.08^{++}$ & .09 & $.10^{++}$ & .02 & -.05 \\
\hline
\end{tabular}

Note: $n=158$ to 163 . One-tailed tests. 
${ }^{\text {a }}$ Inverse transformed. Must be interpreted accordingly.

${ }^{++} p<.20 .{ }^{+} p<.10 .^{*} p<.05 .{ }^{* *} p<.01 .{ }^{* * *} p<.001$.

To explore whether the negative relationship between participants' rating of their best friend's orderliness and recall speed of the orderliness adjectives was affected by the participants' ratings of their own openness, the correlations presented in Table 20 were presented as a function of whether participants rated themselves as low $(n=54)$, medium $(n=55)$, or high $(n=48)$ on the direct measure of openness (outliers for the rate of approach identified earlier were excluded). Results presented in Table 22 showed that the relationship between conscientiousness and orderliness and the rate of approach differed slightly depending on participants' rating of their openness. In particular, at a low level of openness, the direction of the relationship for conscientiousness was negative $(r=-.15)$, but was close to zero for orderliness $(r=-.04)$; at a medium level, it was negative for conscientiousness $(r=-.20)$ and orderliness $(r=-.20$; similar to what was observed in Study 1); at a high level, it was close to zero for conscientiousness $(r=-.04)$ and negative for orderliness $(r=-.20)$. These results hint that the level of participants' openness may have interfered with recall speed of the orderliness adjectives. To test that idea formally, the estimated rate of approach parameter for the other-referenced adjectives was analyzed in a one-way ANCOVA, with self-reported openness (low, medium, high) as a betweensubjects variable and self-reported orderliness as a continuous covariate. The interaction between other-reported orderliness and self-reported openness was not statistically significant, $F(2,151)=.52, \eta^{2}=.01, p=.60$, suggesting that the association between self-reported openness and recall speed did not differ as a function of the participant's rating of their best friend's orderliness. 
Table 22

Zero-Order Correlations Among the Direct, Indirect, and Other-Reference Measures of Orderliness as a Function of Participants' Level of Openness

\begin{tabular}{|c|c|c|c|c|c|c|c|}
\hline $\begin{array}{l}\text { Participant } \\
\text { Openness }\end{array}$ & $\begin{array}{c}\text { Other-Report and } \\
\text { Memory-based Measures }\end{array}$ & 1 & 2 & 3 & 4 & 5 & 6 \\
\hline \multirow[t]{7}{*}{ Low } & 1. Conscientiousness & - & & & & & \\
\hline & 2. Orderliness & $.79^{* * *}$ & - & & & & \\
\hline & 3. Encoding-Orderliness & $.51^{* * *}$ & $.43^{* *}$ & - & & & \\
\hline & 4. Orderliness Recall & $-.13^{++}$ & $-.17^{++}$ & .04 & - & & \\
\hline & 5. Rate of Approach & $-.15^{++}$ & -.04 & -.06 & $-.39^{* *}$ & - & \\
\hline & 6. Inverse Item gain ${ }^{a}$ & $.15^{++}$ & $.22^{+}$ & -.06 & $-.51^{* * *}$ & $.57^{* * *}$ & - \\
\hline & 7. Inverse Item loss ${ }^{a}$ & .04 & $.15^{++}$ & $-.21^{+}$ & $-.18^{+}$ & -.07 & $.24^{*}$ \\
\hline \multirow[t]{7}{*}{ Medium } & 1. Conscientiousness & - & & & & & \\
\hline & 2. Orderliness & $.65^{* * *}$ & - & & & & \\
\hline & 3. Encoding-Orderliness & $.62^{* * *}$ & $.50^{* * *}$ & - & & & \\
\hline & 4. Orderliness Recall & .03 & -.08 & -.07 & - & & \\
\hline & 5. Rate of Approach & $-.20^{+}$ & $-.20^{+}$ & $-.20^{+}$ & $-.16^{++}$ & - & \\
\hline & 6. Inverse Item gain ${ }^{a}$ & -.04 & -.06 & -.03 & $-.49^{* * * *}$ & $.39^{* *}$ & - \\
\hline & 7. Inverse Item loss ${ }^{a}$ & .10 & .00 & -.07 & .11 & $-.20^{+}$ & $-.18^{+}$ \\
\hline \multirow[t]{4}{*}{ High } & 1. Conscientiousness & - & & & & & \\
\hline & 2. Orderliness & $.70^{* * *}$ & - & & & & \\
\hline & 3. Encoding-Orderliness & $.68^{* * *}$ & $.58^{* * *}$ & - & & & \\
\hline & 4. Orderliness Recall & .06 & $.15^{++}$ & .02 & - & & \\
\hline
\end{tabular}




\begin{tabular}{|c|c|c|c|c|c|}
\hline 5. Rate of Approach & -.04 & $-.20^{+}$ & -.06 & -.01 & - \\
\hline 6. Inverse Item gain ${ }^{a}$ & $-.18^{++}$ & $-.15^{++}$ & $-.22^{+}$ & $-.35^{* *}$ & $.53^{* * *}$ \\
\hline 7. Inverse Item loss ${ }^{a}$ & -.02 & -.07 & .04 & -.12 & -.01 \\
\hline
\end{tabular}

Note: $n=51$ to 55 . One-tailed tests.

${ }^{\text {a }}$ Inverse transformed. Must be interpreted accordingly.

${ }^{++} p<.20{ }^{+} p<.10 .^{*} p<.05 .^{* *} p<.01 .^{* * * *} p<.001$.

Nevertheless, it was noteworthy that the pattern of interrelationships in Table 22 among the orderliness measures and rate of approach to asymptote was similar to that observed in Study 1 at the medium level of participants' openness. That is, the direct measures of conscientiousness, orderliness, and encoding-orderliness were negatively associated with the rate of approach parameter with a similar magnitude $(r \mathrm{~s}=-.20)$, and item gain and loss were not associated with the direct measures. In order to compare the time course of recall to that of Study 1 at the medium level of participants' openness, Figure 13 plots cumulative recall for the other-reference task separately for those who rated their friend as low $(n=17)$, medium $(n=22)$, or high $(n=16)$ on the direct measure of orderliness. Interestingly, the qualitative pattern of cumulative recall appears similar to that observed in Study 1 (see Figure 3). Specifically, the high orderliness group had the nominally lowest cumulative recall at minute 1 , but over the recall period, their level of cumulative recall eventually matched that of the low and medium orderliness groups (e.g., by minute 13). The fact that the pattern of correlations and cumulative recall are similar to that observed in Study 1 only at the medium level of participants' openness and not at the low or high levels provide some degree of support for the idea that the time 
course of recall for the other-reference task was affected by the participants' perception of their own openness.

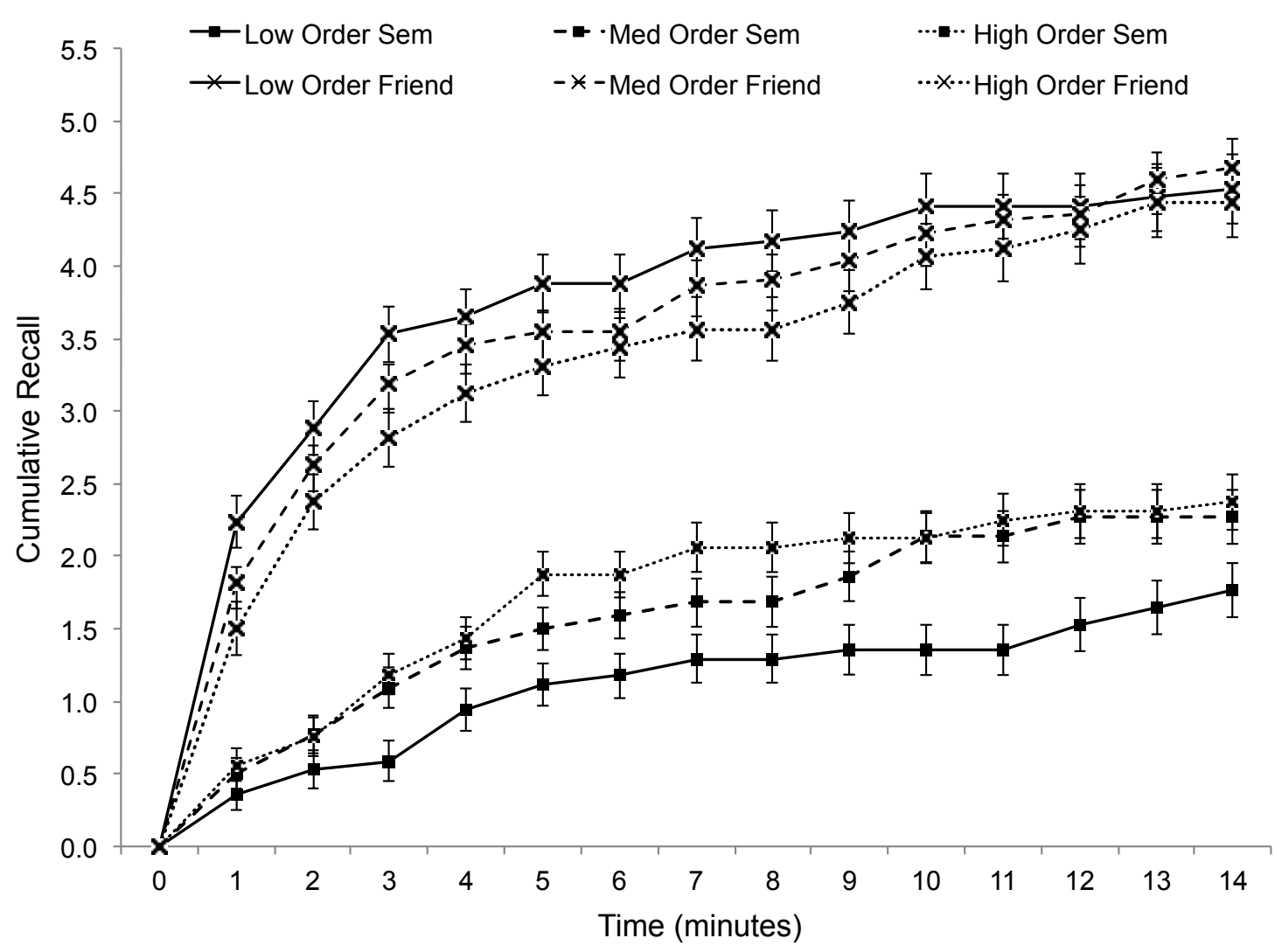

Figure 13. Cumulative recall in Study 3 as a function of orderliness for the medium openness group presented separately for the other-reference and semantic tasks. Error bars represent standard error of the mean. Order $=$ Orderliness. Sem $=$ Semantic. Friend $=$ Other-reference.

Other-report attitudes and behaviour. Correlations were examined to address the hypotheses concerning the predictive validity of the memory-based measures. The self-report items related to conscientiousness and orderliness that were examined in Study 1, and were also collected in the present study, were high school GPA and window cleaning behaviour. The friend-report measures were examined for normality and outliers using the criteria adopted in the previous sections. The high school GPA distribution was 
approximately normal with one outlying case $(z=-4.56)$. The window cleaning distribution was positively skewed, but a log transformation rendered an approximately normal distribution. In the following analyses, outliers identified in the previous section were also excluded. Table 23 presents the zero-order correlations between the personality and memory-based measures and orderliness attitudes.

Table 23

Zero-Order Correlations Between Direct and Other-Reference Measures of Orderliness and Other-Reported Orderliness Attitudes

\begin{tabular}{|c|c|c|}
\hline Friend's Personality Measure & $\begin{array}{l}\text { Friend's High } \\
\text { School GPA }\end{array}$ & $\begin{array}{c}\text { Friend's Window } \\
\text { Cleaning }\end{array}$ \\
\hline 1. Conscientiousness & $.44^{* * *}$ & $.19^{*}$ \\
\hline 2. Orderliness & $.29^{* * *}$ & $.27^{* * *}$ \\
\hline 3. Encoding-Orderliness & $.36^{* * *}$ & $.15^{*}$ \\
\hline 4. Orderliness Recall & -.03 & $-.09^{++}$ \\
\hline 5. Rate of Approach & $-.10^{++}$ & .00 \\
\hline 6. Correct Recognition & .01 & $-.16^{*}$ \\
\hline 7. False Recognition & $.13^{+}$ & .00 \\
\hline 8. Inverse Item gain ${ }^{\mathrm{a}}$ & $-.09^{++}$ & $.17^{*}$ \\
\hline 9. Inverse Item loss ${ }^{\mathrm{a}}$ & .01 & .03 \\
\hline
\end{tabular}

Note. $n=156$ to 158 . Significance tests are one-tailed.

${ }^{\text {a }}$ Inverse transformed. Must be interpreted accordingly.

${ }^{++} p<.20 .{ }^{+} p<.10 .{ }^{*} p<.05 .{ }^{* * *} p<.001$.

Participants' ratings of their friend's high school GPA were significantly positively correlated with their ratings of their friend's conscientiousness, orderliness, and encoding-orderliness. Furthermore, the ratings of high school GPA were marginally correlated with higher false recognition and approaching a marginal correlation with 
slower recall speed and higher item gain. The trends toward slower recall speed and higher item gain provide a certain degree of support for the predictive validity of the memory-based measures. The marginal positive relationship between false recognition and high school GPA is in the opposite direction than what was expected (i.e., lower false recognition implies higher orderliness, which should predict higher GPA). Similarly, although higher ratings of participants' friend's conscientiousness, orderliness, and encoding-orderliness were associated with higher ratings of participants' friend's window cleaning behaviour at home, the significant negative relationship with correct recognition, item gain, and the marginal negative association with orderliness recall are all in the opposite direction than what was predicted. Hence, although there were trends in the predicted direction in support of predictive validity for the rate of approach and item gain measures, there was a lack of consistency in the pattern of associations across the two behavioural indicators, suggesting overall low levels of predictive validity (and small effect sizes).

\section{Discussion}

Study 3 combined the self-reference and semantic task implemented in Study 1 and Study 2 with an other-reference task in a within-subjects design. Specifically, participants self-referenced the openness adjectives from Study 2, other-referenced the orderliness adjectives from Study 1, and semantically encoded a new set of adjectives describing trust. There was a clear self-reference effect, wherein self-referencing elicited a higher proportion recalled and correct recognition than did other-referencing and encoding adjectives semantically. Furthermore, there was also a clear other-reference effect, wherein other-referencing rendered a higher proportion recalled and correct 
recognition than did encoding adjectives semantically. Analysis of item gain and item loss suggested that these effects were due to higher levels of elaborative and organizational processing, supporting conclusions made in Symons and Johnson's (1997) meta-analysis. The analyses of item gain, item loss, and rate of approach, however, were equivocal in explaining why the self-reference task produced higher recall than did the other-reference task. Examination of the time course of recall suggested that the selfreference task may have elicited organizational processing that more effectively narrowed the search of memory for item-specific information, producing approximately one-half of the recall advantage by minute 1 and the other half by minute 6 .

The results examining individual differences in memory performance were mixed, in that the hypothesized negative relationship between recall speed and openness that was observed in Study 2 did not replicate, but the tendency for the direct measures to be related to higher recall of the openness adjectives did replicate. Furthermore, the hypothesized negative relationship between recall speed and orderliness that was found in the self-reference task of Study 1 was successfully extended to the other-reference task. Analyses of divergent validity supported the idea that the failure to replicate the openness-recall speed association observed in Study 2 was due to the within-subjects design, wherein participants were required to tap into their self-concept and their concept of one of their best friends within the same memory task. In particular, participants who rated their best friend as highly orderly recalled the self-referenced openness adjectives faster and other-referenced orderliness adjectives slower than did their counterparts, suggesting there may have been a trade-off in recall of the self- and other-referenced adjectives. When correlations among openness and self-reference memory performance 
were examined separately at low, medium, and high levels of participants' best friend's orderliness, the pattern of interrelationships suggested their opinion of their friend's orderliness moderated the openness-recall speed relationship. Indeed, an ANCOVA on the rate of approach parameter found a marginally significant moderation (interaction) effect. Further support was noted in that the correlations between openness and the memory-based measures at the medium level of their best friend's orderliness were similar to that observed in Study 2, as was the qualitative pattern of cumulative recall for self-referenced adjectives among the low, medium, and high openness groups (i.e., comparing Figure 5 and Figure 11).

The analyses of divergent validity of the memory-based measures for the otherreference task also supported the trade-off hypothesis. In particular, when correlations among orderliness and other-reference memory performance were examined separately as a function of whether participants considered themselves low, medium, or high on the direct measure of openness, the pattern of interrelationships hinted that their own level of openness may have moderated the orderliness-recall speed relationship, although a formal inferential test did not support that conclusion. Nevertheless, the pattern of correlations between the memory-based measures of the other-reference task and orderliness at the medium level of participants' openness were similar to that observed in Study 1, as was the qualitative pattern of cumulative recall for the other-referenced adjectives among the low, medium, and high orderliness groups (i.e., comparing Figure 3 and Figure 13).

The set of additional analyses examining the potential trade-off in recall speed showed that the hypothesized pattern of cumulative recall that was found in Study 1 and 
Study 2 could be observed in Study 3 most clearly at the medium level of the opposing trait (i.e., medium level of friend's orderliness when examining self-reference recall speed, and medium level of participant's openness when examining other-reference recall speed). Lastly, the memory-based measures derived from the self- and other-reference memory performance exhibited weak predictive validity of the criterion attitudes and behaviours.

\section{General Discussion}

The present research was inspired by Rogers et al.'s (1977) classic memory study that demonstrated a recall advantage on a surprise memory test for personality trait adjectives that were encoded in reference to the self relative to adjectives that were encoded in other ways (e.g., semantically). They argued that this self-reference effect stemmed from relating the adjectives to the self-concept, which afforded a rich source of elaborative processing upon encoding. Memory researchers have further described elaborative processing as providing item-specific information (i.e., idiosyncratic retrieval cues) that supports recall and recognition of encoded items (Burns, 1993; Burns \& Hebert, 2005; Einstein \& Hunt, 1980; Hunt \& Einstein, 1981; Hunt \& McDaniel, 1993; McCabe et al., 2004; see also Craik \& Tulving, 1975). I argued that the compelling question of whether the self-concept as a memory structure in fact provides more elaborate processing in support of recall has not been adequately tested. The main goal of the present research was therefore to address this question by examining whether individual differences in personality self-concept are associated with more elaborate processing following self-reference encoding, when the trait adjectives were congruent 
with that memory structure (i.e., only when self-referenced adjectives matched a personality dimension).

Further work conducted after Rogers et al. (1977) determined that under certain conditions organizational processing alone could account for the self-reference effect (Klein \& Kihlstrom, 1986), in that self-referencing provided a means to organize the encoded material into categories (e.g., words that describe me vs. those that do not), something other comparison encoding tasks had not encouraged. However, using the additive effects logic of Einstein and Hunt (1980), Klein and Loftus (1988) showed that both elaborative and organizational processing were important factors in explaining the self-reference effect, a conclusion that was supported in a meta-analytic review using the same approach (Symons \& Johnson, 1997). Thus, the literature to date suggested that elaborative and organizational processing are responsible for the self-reference effect.

One major impediment to the elaborative processing explanation of the selfreference effect, or levels-of-processing in general, has been to obtain a measure of elaboration that is independent from the to-be-explained phenomenon. Baddeley (1978) and Eysenck (1978) were quick to point out that it is circular to claim that elaborative processing causes the higher recall observed in certain encoding conditions, when elaborative processing is evidenced by the higher amount recalled. In an effort to obtain measures of elaborative and organizational processing that were independent from the total amount recalled, Klein et al. (1994) examined the impact of self-reference encoding on free recall using a repeated-testing paradigm. They found that self-referencing produced fewer item losses and marginally more item gains on the second recall trial than did semantic encoding. These results suggested that self-reference elicited relational 
information that was effective at preventing item loss (either by way of a retrieval plan or common category information; e.g., Raajimakers \& Shiffrin, 1981; Slamecka, 1968) and item-specific information that allowed for item gain in the second recall trial (idiosyncratic retrieval cues were not exhausted by the end of the first 5 minute recall trial).

The work of Burns (1993; Burns \& Hebert, 2005; Burns \& Schoff, 1998) convincingly demonstrated that item gain changes dramatically depending on how much time has been provided for recall, limiting it as a measure of item-specific processing. The authors proposed examining cumulative recall curves to circumvent the problem (Bousfield \& Sedgewick, 1944; see Wixted \& Rohrer, 1994 for an excellent review), with the rate of approach to asymptote providing insight into the amount of item-specific processing engaged during encoding. Burns and Hebert (2005; see also Burns \& Schoff, 1998) suggested examining a plot of cumulative recall in addition to the rate of approach to asymptote to better understand how the complex interplay of item-specific and relational information has affected recall. The novel approach taken to address the main research question of the present studies was to combine the repeated-testing paradigm with a cumulative recall curve analysis of self-reference memory performance using measures of item-specific and relational processing that were largely independent of amount recalled. The main hypothesis was that participants who were high on a personality trait that matched the self-referenced trait adjectives would recall those adjectives more slowly than their counterparts, suggesting that they engaged in more elaborative processing (i.e., encoded more item-specific information). 


\section{The self- and other-reference effects}

Before discussing the personality differences in memory performance, let us examine the overall free recall and then recognition memory results. Study 1 and 2 confirmed a strong levels-of-processing effect on free recall, wherein self-reference encoding rendered a higher proportion recalled than did semantic encoding, which in turn produced a higher proportion recalled than the structural and rhyming tasks. Study 3 also confirmed a strong levels-of-processing effect, wherein self-reference encoding yielded a higher proportion recalled than did other-reference encoding, which in turn produced higher recall than did the semantic task. Thus, the three studies replicated the selfreference and other-reference effects on proportion recalled (Symons \& Johnson, 1997).

\section{Memory processes responsible for the self- and other-reference effects}

The item gain, item loss, and cumulative recall results help to understand the contributions of elaborative and organizational processing in the self- and other-reference effects. The self-reference task in Study 1 and Study 3 produced higher item gain than did the semantic task, whereas the difference in Study 2 was not significant. The logtransformed rate of approach to asymptote for the self-reference task, however, was higher in Study $2(M=.33,95 \%$ CI $[.26, .39])$ than it was in Study $1(M=.06,95 \%$ CI [$.24, .36])$ and Study $3(M=-.28,95 \%$ CI $[-.35,-.22])$, suggesting that encoded relational information may have produced faster recall in Study 2. Indeed, an examination of cumulative recall for the semantic and self-reference tasks showed that most of the recall advantage occurred at minute 1 and 2 (see Figure 4), whereas an examination of cumulative recall in Study 1 and 3 showed that the recall advantage persisted across the recall period (see Figure 2 and Figure 9). Hence, it is possible that the item gain measure 
in Study 2 was not significant because the additional encoded item-specific information in the self-reference task produced successful retrieval of list items earlier in the recall period (i.e., before minute 5) due to the deployment of more effective relational information (Burns \& Hebert, 2005; Burns \& Schoff, 1998). The item loss data corroborate this explanation, in that the nominal rate of item loss for self-referentially encoded adjectives was lower in Study $2(M=.09,95 \%$ CI $[.07, .12])$ than it was in Study $1(M=.13,95 \%$ CI $[.09, .17])$ and Study $3(M=.14,95 \%$ CI $[.11, .18])$. The otherreference task in Study 3 produced higher item gain than did the semantic task indicating that the other-reference effect was driven in part by elaborative processing. In contrast, the self- and other-reference tasks did not differ in item gain, suggesting the difference in recall was not due to item-specific information, a point I return to shortly.

Turning to the proportion of item loss on the second recall trial, the results showed that self-referentially encoded adjectives in Study 1 and Study 2 yielded significantly lower item loss than did semantic encoding, and the difference in Study 3 was marginally significant. These results replicate Klein et al.’s (1994; Experiment 1) item loss finding and agree with the conclusion reached by Symons and Johnson's (1997) meta-analysis that the self-reference effect was driven in part by more effective relational information acquired through organizational processing. The other-reference task in Study 3 elicited significantly lower item loss than did the semantic task, supporting Symons and Johnson's (1997) conclusion that the other-reference effect was also in part due to more effective relational information. In contrast, the difference in item loss between the self- and other-reference tasks was not significant, suggesting that the recall 
advantage of self-referencing over other-referencing (of a best friend) was not due to organizational processing.

Given that item gain, item loss, and rate of approach to asymptote did not differ between the self- and other-reference tasks, why did self-referencing elicit higher recall than other-referencing? The pattern of cumulative recall hinted that self-reference might have provided more effective relational information, in that approximately one-half of the recall advantage was evidenced by minute 1 and the remainder was realized by minute 6 (see Figure 9). However, relational processing differences typically impact cumulative recall very early on (e.g., minutes 1 and 2; Burns \& Schoff, 1998), whereas there seemed to be a somewhat gradual separation of the curves until minute 6 . It is possible that this gradual increase was due to item-specific information, but that the relational information effectively narrowed the search of memory and produced successful retrieval earlier in the time course of recall than usual. A way to explore this possibility in future research is to provide more time for recall in order to allow item-specific processing differences to surface beyond the 14 minutes provided in Study 3 (i.e., Figure 9 shows that recall has not reached asymptote for either task). Another approach is to increase the list length (i.e., more trait adjectives) in order to exaggerate any item-specific processing differences between the two tasks. For example, Rohrer and Wixted (1994; Experiment 2) found that a longer list produced slower recall relative to a shorter list, implying that more itemspecific information was engaged when encoding a longer list of items. If self-reference indeed produces more item-specific information than does the other-reference task used here, increasing the list length should slow down recall more for self-referenced items than for other-referenced items, because of a disproportionate increase in encoded item- 
specific information in the self-reference task (i.e., exaggerating rate of approach differences). Further research would be required to explore possibilities such as these.

In terms of the recognition test performance, the levels-of-processing and selfreference effects on correct recognition were robust across the three studies, and Study 3 revealed an other-reference effect on correct recognition. In contrast, the rates of false recognition for the self-reference and semantic lures in Study 2 were equivalent, whereas in Study 3 there was a lower rate of false recognition for self-reference lures than the semantic and other-reference lures. The correct and false recognition results taken together suggest that the processing that occurred in the self-reference task in Study 3 produced item-specific and relational information that was more distinctive (i.e., allowed for a high level of correct recognition and better rejection of lures), whereas in Study 2 the item-specific and relational information was less capable of successfully differentiating lures from previously encoded items. ${ }^{26}$

One explanation for the difference in false recognition between studies is that the self-reference task elicited more effective relational information in Study 2 than it did in Study 3, which served to increase the rate of false recognition (e.g., Hege \& Dodson, 2004). Free recall performance discussed earlier supports that explanation, in that the rate of approach to asymptote was lower and item loss was higher in Study 3 than it was in Study 2, indicating that more relational information was encoded in Study 2. A plausible alternative explanation that cannot be ruled out with the present data is that more itemspecific information was encoded in Study 3 than it was in Study 2, which has been shown to elicit lower levels of false recognition (Einstein \& Hunt, 1980; McCabe, et al.

\footnotetext{
${ }^{26}$ False recognition in Study 1 could not be examined as a function of encoding task because the list items and lures were all selected from the same superordinate category (i.e., conscientiousness). Hence, lures associated with the semantic task were equally good lures for the self-reference task.
} 
2004). Consistent with that explanation is the slower rate of approach and nominally higher item gain in Study 3. Of course, there are other potential differences between the two studies that may explain the different false recognition results (e.g., 10 vs. 14 minute recall test length and 13 vs. 10 list adjectives in Study 3 relative to Study 2).

Regardless of differences in recognition test performance between the studies, the data were likely contaminated by a testing effect, which makes for tenuous conclusions about the processing that was engaged during self-reference. For example, research on the testing effect conducted by Chan and McDermott (2007; Experiment 3) found that engaging in free recall in a testing condition, relative to studying the list items in a no testing condition, prior to taking a recognition test increased the rate of correct recognition and affected the processing involved in making recognition decisions. Specifically, engaging in retrieval during free recall served to increase the contribution of conscious recollection of list items when making subsequent recognition decisions, while leaving the relatively automatic contribution of familiarity unaffected (Jacoby, 1991). Given that the encoding manipulation in the present studies is known to affect the activation of item-specific and relational information in memory and that retrieval of list items during the two free recall tasks likely impacted recognition decisions, it is not clear how differences in recollection and familiarity may have obscured the contributions of item-specific and relational information during the subsequent recognition test.

Furthermore, it is not clear whether engaging in retrieval prior to the recognition test interacted with the encoding conditions; that is, whether retrieval of items disproportionally affected recollection and familiarity of semantically and selfreferentially encoded items. If participants were given the recognition test before 
engaging in free recall, research suggests that the pattern of data would likely appear different and could lead to different conclusions. Given that the main purpose of the present research was to link validated personality trait measures to free recall performance, and that the recognition data served as a secondary indicator of itemspecific processing, the focus of the discussion moving forward will be on free recall performance.

The most consistent explanation for the free recall performance across the studies is that elaborative and organizational processing produced the self- and other-reference effects, as was predicted (Klein \& Loftus, 1988; Klein et al., 1994; Symons \& Johnson, 1997). The organizational processing elicited relational information that more effectively narrowed the search of memory and the elaborative processing rendered item-specific information that elicited successful retrieval of more list items from within the narrowed search set. It is entirely possible that the self-concept as a memory structure has the capacity to invoke more item-specific information and effective relational information during self-reference encoding than do other memory structures, including that comprising the personality of a best friend. Although Rogers et al. (1977) may not have anticipated that relational information contributes to the self-reference effect (Klein \& Kihlstrom, 1986), their explanation (or rather, hypothesis) that the self-concept provides rich item-specific information through elaborative processing brings us to a discussion of the results that address the main objective of the present thesis.

\section{Personality and self-reference recall speed}

In particular, the focal goal of Study 1 and 2 was to examine whether individual differences in personality self-concept are associated with the encoding of more item- 
specific information during self-reference of corresponding trait adjectives, closely following Rogers et al.'s (1977) procedure. It was hypothesized that participants who viewed themselves as highly orderly (Study 1) and highly open to experience (Study 2) would encode more item-specific information when making self-reference decisions, which would counter-intuitively slow down free recall speed. Furthermore, it was expected that self-reference judgments would be necessary for the negative personalityrecall speed relationships to emerge (i.e., they would not emerge among semantically encoded items), and that the pattern would only emerge when the trait adjectives encoded in the self-reference task matched a corresponding personality measure (i.e., orderliness in Study 1 and openness in Study 2).

The results favoured the negative personality-recall speed hypothesis, and between the two studies, there was an uncrossed double dissociation (Dunn \& Kirsner, 1988) in self-reference recall speed between the target and non-target traits (i.e., orderliness and openness). Specifically, in Study 1, participants who were high on the direct measure of conscientiousness and orderliness (and who endorsed more orderliness adjectives as self-descriptive) recalled the self-referenced orderliness adjectives more slowly over the 10-minute recall period than did their counterparts $(r=-.23)$. In contrast, in Study 2, when a new list of adjectives describing openness was self-referenced, the correlation between orderliness and recall speed was small and not significant $(r=.03)$. Moreover, when comparing the pattern of cumulative recall as a function of orderliness in Study 1 versus Study 2 (i.e., comparing Figure 3 and Figure 8), it was apparent that slower recall of the high orderliness group that occurred in Study 1 did not also occur in Study 2 (i.e., in Study 2, the high orderliness group had the nominally highest recall from 
minute 2 onwards, whereas in Study 1 it was not until minute 8 that they achieved nominally higher recall). Furthermore, additional estimates of cumulative recall curves for the low, medium, and high orderliness groups uncovered that the low and medium groups had a similar rate of approach in Study 1 (i.e., low: $\lambda=.82,95 \%$ CI $[.60,1.05]$ vs. medium: $\lambda=.88,95 \%$ CI $[.61,1.15])$ and Study 2 (i.e., low: $\lambda=.59,95 \%$ CI $[.45, .73]$ vs. medium: $\lambda=.53,95 \%$ CI $[.38, .69])$, whereas recall speed for the high orderliness group was slower in Study $1(\lambda=.49,95 \%$ CI $[.37, .61])$ than in Study $2(\lambda=.60,95 \%$ CI $[.52$, $.68])$. This first dissociation supported hypotheses of convergent and divergent validity of the orderliness-recall speed measure following self-reference encoding, and it suggested that the observed pattern in Study 1 was not simply due to orderly (i.e., slow and careful) behaviour per se.

The second dissociation was observed when examining the relationship between openness and self-reference recall speed. Specifically, participants who were high on the direct measure of openness in Study 2 recalled the openness adjectives more slowly over the 10-minute recall period than did their counterparts $(r=-.24)$. In contrast, in Study 1, the correlation between openness and recall speed of the orderliness adjectives was smaller and not significant $(r=-.12)$. Importantly, although the pattern of cumulative recall was very similar between the studies for the low and medium openness groups (potentially explaining the small negative correlation observed in Study 1), the high openness group was clearly much slower in Study 2 than in Study 1 (i.e., Figure 5 vs. Figure 6). Furthermore, additional group-level estimates of cumulative recall curves supported that observation, with the low and medium openness groups approaching asymptote at similar rates in Study 1 (low: $\lambda=.62,95 \%$ CI $[.43, .80]$ vs. medium: $\lambda=$ 
$.68,95 \%$ CI $[.52, .83]$ ) and Study 2 (low: $\lambda=.61,95 \%$ CI $[.45, .76]$ vs. medium: $\lambda=.69$, $95 \%$ CI $[.56, .82])$, whereas the high openness group approached asymptote much slower in Study $2(\lambda=.45,95 \%$ CI $[.37, .53])$ than in Study $1(\lambda=.87,95 \%$ CI $[.58,1.16])$. This second dissociation supported the convergent and divergent validity of the opennessrecall speed measure following self-reference, and it extended the findings of Study 1 to a different Big Five trait. Although the uncrossed double dissociation was not demonstrated within the same sample (i.e., study) and personality was an observed (i.e., not manipulated) variable, the results nevertheless support the hypotheses of convergent and divergent validity, indicating that a correspondence between the direct personality measure and the encoded trait adjectives may be necessary to observe the negative personality-recall speed relationship. Stated differently, Study 2 can be thought of as demonstrating a conceptual replication of the negative personality-recall speed relationship across personality traits.

A plausible alternative explanation is that these findings were due to differences in the processing of relational information. That is, slower recall among those who were high on a trait may have been due to the use of less effective relational information during retrieval. However, in Study 1, item loss of the self-referenced adjectives was not related to the direct measures of orderliness, conscientiousness, or encoding-orderliness $(r \mathrm{~s}<.06)$, nor was item loss associated with recall speed $(r=-.01)$. Similarly, in Study 2 , item loss was not related to the direct measure of openness $(r=-.07)$ or recall speed $(r=$ .08). These results do not support the alternative explanation. In fact, these findings are particularly important given that the differences in cumulative recall among the orderliness groups in Study 1 were most clearly observed early in the first recall task, 
where relational information would have had its most notable impact on cumulative recall.

A possible counter-argument is that correlations with item loss were not significant because item loss was simply not a good indicator of relational information, especially in light of the relatively low level of cumulative recall that was observed in the studies (i.e., a potential floor effect). However, item loss was sensitive enough to detect the hypothesized encoding task differences in each study (i.e., semantic vs. selfreference) and the observed proportion of item loss for self-referenced adjectives in Study $1(M=.13)$, Study $2(M=.09)$, and Study $3(M=.14)$ was similar to that observed in Klein et al. (1994; $M=.13)$, whose participants had a nominally higher level of recall at the end of the first recall trial, thus more opportunity to forget items on the second trial ( $M=5.5$ adjectives vs. $M \mathrm{~s} \sim 3.5$ to 4.5 adjectives in the present studies). Thus, the results suggest that item loss tapped relational processing and that relational processing was not responsible for the negative personality-recall speed relationships that were observed. However, an important objective for future research would be to increase the amount recalled by the end of the first recall trial (e.g., by increasing the recall test length or list length) in order to allow an opportunity for more item loss on the second recall trial and rule out the alternative explanation of relational processing differences.

\section{Personality and other-reference recall speed}

Study 3 explored the possibility that a memory structure other than the selfconcept could elicit the negative personality-recall speed relationship. Specifically, one goal of Study 3 was to extend the orderliness-recall speed association observed in Study 1 to participants' concept of their best friend's orderliness. A second goal was to 
simultaneously replicate the negative openness-recall speed association following selfreference observed in Study 2. The within-subjects design allowed for a test of uncrossed double dissociation within the same sample, with the expectation that participants' ratings of their best friend's orderliness (but not their own openness) would be negatively related to other-reference recall speed and that participants' openness (but not their view of their best friends' orderliness) would be negatively related to self-reference recall speed.

The personality and recall speed results were mixed, in that the hypothesized negative openness-recall speed association did not replicate, but the hypothesized negative orderliness-recall speed association was successfully extended to the otherreference task. Additional analyses examining divergent validity suggested that the within-subjects design that required participants to bring to mind their own personality and the personality of a best friend might have been responsible for the failure to replicate. In support of that idea, there was a marginally significant moderation effect of participants' ratings of their best friend's orderliness on the openness-recall speed association. In particular, the pattern of interrelationships between openness and selfreference memory measures, as well as the pattern of cumulative recall, that most closely matched that observed in Study 2 was obtained only at the medium level of participants' best friend's orderliness (the rate of approach was faster $r=.16$ at the low level, slower $r$ $=-.14$ at the medium level, and null $r=.02$ at the high level). These results hint that there might have been less retrieval interference (or competition) from the item-specific and relational information elicited by the other-referenced adjectives when participants did not view their friend as particularly orderly (i.e., the more natural time course of recall proceeded when participants did not view their friend as particularly orderly). A second 
piece of evidence in support of this trade-off hypothesis was that participants who viewed their friend as highly orderly recalled the other-referenced orderliness adjectives more slowly (as was predicted) but recalled the self-referenced openness adjectives more quickly than did their counterparts. The trade-off was asymmetric, in that the moderation effect of participants' openness on the relationship between orderliness and otherreference recall speed was not significant. When the correlations were examined as a function of openness, the negative orderliness-recall speed association could be observed at the low $(r=-.13)$ and medium $(r=-.20)$ level of participants' openness, but not the high level $(r=-.04)$. Hence, participants' own level of openness did not seem to interfere with recall speed in the same way as their view of their best friend's orderliness.

It is important to note that the choice of experimental design has been shown to affect whether certain memory phenomena will be observed. For example, Greene (1996) demonstrated that the impact of a retention interval (i.e., the time between learning a list and a free recall test) on the proactive interference observed in the Brown-Peterson paradigm (i.e., the progressively poorer recall of random three letter strings, selected from a fixed set of letters, presented in a series of trials) was eliminated with the use of a between-subjects design instead of a within-subjects design. The impact of bizarre imagery elicited by stimulus materials (i.e., bizarre vs. common sentences) on free recall can be observed in a within-subjects design, but not a between-subjects design (McDaniel \& Einstein, 1986). Another example is the generation effect, wherein better recall for self-generated material than perceived (i.e., heard or read) material is typically only observed in a within-subjects design, whereas a reversal of the generation effect (i.e., better memory for perceived than self-generated material) is typically only observed in a 
between-subjects design (Mulligan \& Duke, 2002). These results have had important theoretical implications and have stimulated further research to determine why the design is important for eliciting the phenomena.

In contrast, in the present studies, the theoretical implications from a memory perspective of whether the negative personality-recall speed association was affected by the design may be less interesting, as the dependent variable of interest (i.e., recall speed) was likely obscured by the fact that participants were forced by the task to choose to recall self-referenced or other-referenced adjectives first (i.e., simultaneous recall of both types of adjectives was not possible). Given that both tasks elicited relational information, research suggests responding was likely clustered as a function of encoding task. Indeed, Burns, Hwang, and Burns (2011) found that when they used a withinsubjects design and blocked stimuli by orienting task (as was done in the present experiments), participants tended to cluster their recall as a function of the orienting task, which obscured their patterns of cumulative recall. In a between-subjects design, participants would not be required to prioritize, and the natural time course of recall driven only by the item-specific and relational information elicited in the self-concept (or the concept of a best friend) would be observed. In a similar vein, the personality-recall speed associations in the three studies were likely also somewhat obscured by having to recall adjectives encoded in the semantic and shallow tasks in the same recall period. Aside from the issues concerning the research design, it is interesting that there seemed to be an asymmetric trade-off favouring participants' view of their best friend.

One possible explanation for the asymmetric trade-off was the focus that was placed on the best friend at the start of the experimental procedure. Just prior to the 
levels-of-processing task, participants were asked to bring to mind a best friend and then complete a brief questionnaire about this specific individual. They were also informed that they would need to make judgments about the best friend at times during the experiment. It is possible that this focus on the best friend induced a priority to recall other-referenced adjectives before self-referenced ones, but only under certain combinations of self- and other-relevance of the trait adjectives (e.g., when participants viewed the orderliness adjectives as particularly relevant to the personality of their best friend).

\section{Why did open participants recall more adjectives?}

A relatively consistent result across the studies was that openness was associated with higher recall. To summarize, openness was positively associated with higher recall of the self-referenced orderliness adjectives $(r=.31)$ in Study 1, but not recall of the adjectives encoded in the combined tasks (see Figure 6). In Study 2, there was a marginal positive association between openness and recall of the self-referenced openness adjectives $(r=.20)$, and there was a trend toward higher recall of the adjectives in the combined tasks (see Figure 5). In Study 3, openness was again positively associated with recall of the self-referenced openness adjectives $(r=.26)$, but not recall of the adjectives from the combined tasks (see Figure 10). Furthermore, open participants recalled more of the other-referenced adjectives than did their less open counterparts $(r=.16)$. Thus, openness was generally associated with higher levels of recall, but this was not necessarily limited to the self-reference task or to adjectives describing openness.

An examination of cumulative recall across the studies showed that the recall difference among the low, medium, and high openness groups occurred early in the recall 
period (e.g., minutes 1 through 3; see Figure 6 and Figure 10), suggesting that open participants utilized more effective relational information in the search of memory than did their less open counterparts. There was no evidence that the recall advantage stemmed from gaining items later in the recall period, except when the openness adjectives were self-referenced in Study 2 (but that was predicted). Thus, the recall advantage did not appear to be due to increased item-specific information, but instead seemed to be due to use of more effective relational information. It may be that open participants engage with the material differently in general than less open participants, wherein they tend to focus more of inter-item similarity (relational information), sometimes providing more effective relational information to narrow the search of memory. However, further research would be required to test that possibility.

The most directly relevant study concerning openness and recall was conducted by Katz (1987), who examined the relationship between creativity, measured by a remote association test (RAT), and free recall following structural, semantic, and self-reference encoding of creative-relevant and creative-irrelevant trait adjectives. In the first study, Katz reported that participants who scored high on the RAT recalled more creativerelevant than creative-irrelevant adjectives following self-reference, but not following structural or semantic encoding, and not among those who scored low on the RAT (i.e., a three-way interaction). The second study excluded the structural and semantic encoding tasks and focused on recall of self-referenced adjectives. Similar to the first study, participants who scored high on the RAT recalled more creative-relevant than creative irrelevant adjectives, but that was not the case among those who scored low on the RAT. Analyses determined that the free recall effect could not be observed as a function of 
verbal intelligence or self-reference processing time. On the basis of these data, Katz concluded that the self-schema of creative participants produced the selective recall advantage in the self-reference task, arguing that the self-schema allowed for deeper processing (i.e., more elaboration) of the creative-relevant adjectives.

The main conclusion of the present studies was consistent with that put forward by Katz (1987). Interestingly, however, the results are not compatible. Specifically, in Katz's second study, 118 trait adjectives were self-referentially encoded and a 5-minute recall test was administered (the recall test length in the first study was not reported, but 84 adjectives were subjected to the various encoding tasks). Given that self-referencing would have likely elicited far more item-specific information (i.e., elaboration) than that elicited by the 10 or 13 adjectives used in the present studies, a much longer recall test than 5 minutes would have been required to isolate an item-specific processing difference as a function of RAT performance. For example, the slow recall of the high openness group observed in Study 2 of the present thesis exceeded the recall of the low group by minute 2 but did not exceed the recall of the medium group until minute 7 (see Figure 5). Thus, it took approximately 7 minutes for the item-specific difference to produce higher recall. In contrast, as discussed above, relational processing differences as a function of openness appeared much earlier in the recall period (e.g., minutes 1 through 3 ). Hence, it does not seem likely that the recall difference noted in Katz was due to deeper processing (i.e., more elaboration); rather it was likely due to more effective relational information. If that was the case, however, it is not clear why the recall advantage among participants who scored high on the RAT only occurred in the self-reference task. In the present studies, although the item-specific processing difference was isolated to self-referencing 
openness adjectives, the evidence suggested that relational processing differences were responsible for the higher recall observed when self-referencing orderliness adjectives (Study 1) and other-referencing orderliness adjectives (Study 3). Hence, the present results are not consistent with Katz' results, but our conclusions concerning elaboration agree.

Care must be taken, however, in making direct comparisons between openness and the RAT. Harris (2004) found that the modified version of the RAT used in Katz (1987) did not load onto an openness factor $(\lambda=.02)$ that was indicated by the ideas scale of the NEO-PI-R, the sentience and understanding scales of the Personality Research Form (Jackson, 1989), and the breadth of interest, complexity, and innovation scales of the Jackson Personality Inventory (Jackson, 1976; correlations were in the $r=.09$ to $r=$ .22 range), but instead loaded onto an intelligence factor $(\lambda=.63)$ indicated by the spatial, object assembly, information, and vocabulary subscales of the Multidimensional Aptitude Battery (Jackson, 1984; correlations in the $r=.28$ to $r=.41$ range). However, there was a weak correlation between the openness and intelligence factors (i.e., .18). Nevertheless, the openness measure used in the present studies may in fact capture a more attitudinal aspect of dispositional creativity than the cognitive aspect captured by the RAT. Further research using a longer recall period and analysis of cumulative recall would be required to tease apart the contributions of item-specific and relational processing in Katz' work.

Other research in the social cognitive literature has documented that openness is an important factor for autobiographical memory. For example, Rasmussen and Bernsten (2010) reported that openness, as measured by the NEO-FFI (Costa \& McCrea, 1992), 
was positively associated with the use of autobiographical memories to guide thinking, behaviour, and problem solving (a directive function of autobiographical memory) and to define the self and identity (a self function). Although the use of autobiographical memory seems plausible, Klein, Loftus, and Burton (1989; see also Klein, Loftus, Trafton, \& Fuhrman, 1992) have shown that a self-descriptiveness task such as the one used in the present studies (i.e., "Describes Me?”) does not involve autobiographical memory retrieval unlike another commonly used self-reference task in the literature (e.g., "Think of a personal experience in which you manifested the presented trait"). The authors used a task facilitation paradigm to determine if the amount of time to make a self-descriptiveness judgment would decrease if participants were asked to retrieve an autobiographical memory first (and vice-versa). The results showed no facilitation of response times, suggesting that the self-descriptiveness and autobiographical tasks do not share processes (i.e., they are independent). Follow-up work conducted by Klein, Loftus, and Sherman (1993) found facilitation of response times for the self-descriptiveness task when a more generic autobiographic memory was retrieved before making the selfdescriptiveness judgment (e.g., "I enjoy parties" instead of "I enjoyed singing karaoke at John's party last weekend"). It is possible that open participants tend to retrieve more generic autobiographical memories when engaged in self-reference, which could serve as effective relational information. More specifically, a generic autobiographical memory subsumes a whole class of self-relevant behaviour, which could narrow the search of memory considerably. However, it is not clear why open participants would engage in generic autographical retrieval outside of a self-reference task. Nevertheless, exploration 
around this possibility may provide an explanation for the relational processing that was responsible for the higher recall among open participants.

\section{Does recall speed have predictive validity?}

One objective of the present studies was to test the predictive validity of the recall speed measure (rate of approach and item gain). To summarize, the results of Study 1 showed that slower recall of the orderliness adjectives was associated with significantly higher self-reported window cleaning behaviour and marginally fewer omission errors during the $\mathrm{d} 2$ test of concentration and attention. Item gain in the second recall trial was significantly associated with fewer omission errors in the $\mathrm{d} 2$ test, as well as marginally less lateness to the experimental session. The relationships with the other indicators of conscientiousness were smaller and not significant (i.e., the week within the semester, participants' arrival time, commission errors in the $\mathrm{d} 2$ test, and high school GPA). Thus, orderliness recall speed and item gain were associated with some of the behavioural and attitudinal measures of conscientiousness, but the associations were fairly weak (e.g., $|r s|$ $=.15$ to .29$)$.

Study 2 found that slower recall of the openness adjectives was associated with a lower probability of voting conservative and with taking a liberal arts degree. Participants who took the debriefing sheet at the end of the experimental session had significantly more item gains. Participants who indicated that they enjoy reading classic literature tended to have higher item gain in the second recall task $(p<.20)$. Higher self-reported general intelligence was associated with fewer item gains, which was in the opposite direction than was expected. The associations with the other behavioural and self-report measures of openness and imaginativeness (i.e., spontaneous curiosity, romantic partner 
who is open to experience, daydream duration, daydream vividness, and playing a musical instrument) were not statistically significant. Hence, openness-recall speed and item gain were only associated with some of the behavioural and attitudinal measures, and again the associations were fairly weak (e.g., $|r s|=.28$ to .29 ).

The results of Study 3 showed that self-reference recall speed was not significantly associated with any of the indicators of openness attitudes and behaviours. However, slower recall was marginally associated with higher self-reported general intelligence $(r=-.12)$, but was marginally associated with taking a non-liberal arts degree (which is in the opposite direction than was expected). Higher item gain was significantly associated with higher self-reported general intelligence. For the other-reference task, slower recall speed of the orderliness adjectives tended to be associated with higher reports of participants' best friend's high school GPA, but higher item gain was associated with lower ratings of participants' best friend's window cleaning behaviour, which is in the opposite direction than was expected. It is possible that the predictive validity of the recall speed measure was poorer in Study 3 than it was in Study 1 and Study 2 because it was affected by having to recall items from the self- and otherreference tasks at the same time (i.e., the poorer predictive validity is consistent with the trade-off hypothesis discussed earlier).

Overall, the results across the studies suggest that predictive validity of the recall speed measure was weak. It should be noted, however, that the predictive validity of other indirect measures of personality, such as conscientiousness and openness-IATs, has also been shown to be weak and inconsistent (Back, Schmukle, \& Egloff, 2009; Steffens \& Schulze- König, 2006). The IATs used in the present studies echo that conclusion. For 
example, out of all the tests of predictive validity for the IATs, only two reached statistical significance and they were not consistent between studies: higher implicit openness was associated with taking the debriefing sheet and playing a musical instrument in Study 3 but not in Study 2. Thus, on the whole, the results suggest that the predictive validity of the recall speed measure was actually superior to that of the personality-IATs used in the present studies. Furthermore, several refinements to the procedure may reduce construct-irrelevant variance (e.g., use of a between-subjects design), which could improve predictive validity.

\section{Is the personality-recall speed finding consistent with social-cognitive theories of self-concept?}

Theories of the self that propose a semantic network consisting of linked (associated) concepts could be combined with the assumptions of a basic random search model of memory retrieval to accommodate the present findings in an interesting way. Let us consider Greenwald et al.'s (2002) united theory of attitudes, stereotypes, selfesteem, and self-concept, in which a social knowledge structure is proposed as a semantic network encompassing concepts representing the self, others, attributes, and valence. The self-concept in this theory is the collection of associations between the self and other concepts represented by nodes (e.g., intelligent). Concepts are activated by external stimuli or by the activation of associated concepts. This type of semantic associative network has roots in the memory literature (e.g., Collins \& Quillian, 1969). For illustrative purposes, Figure 14 displays a copy of the social knowledge structure discussed in Greenwald et al. (2002). 


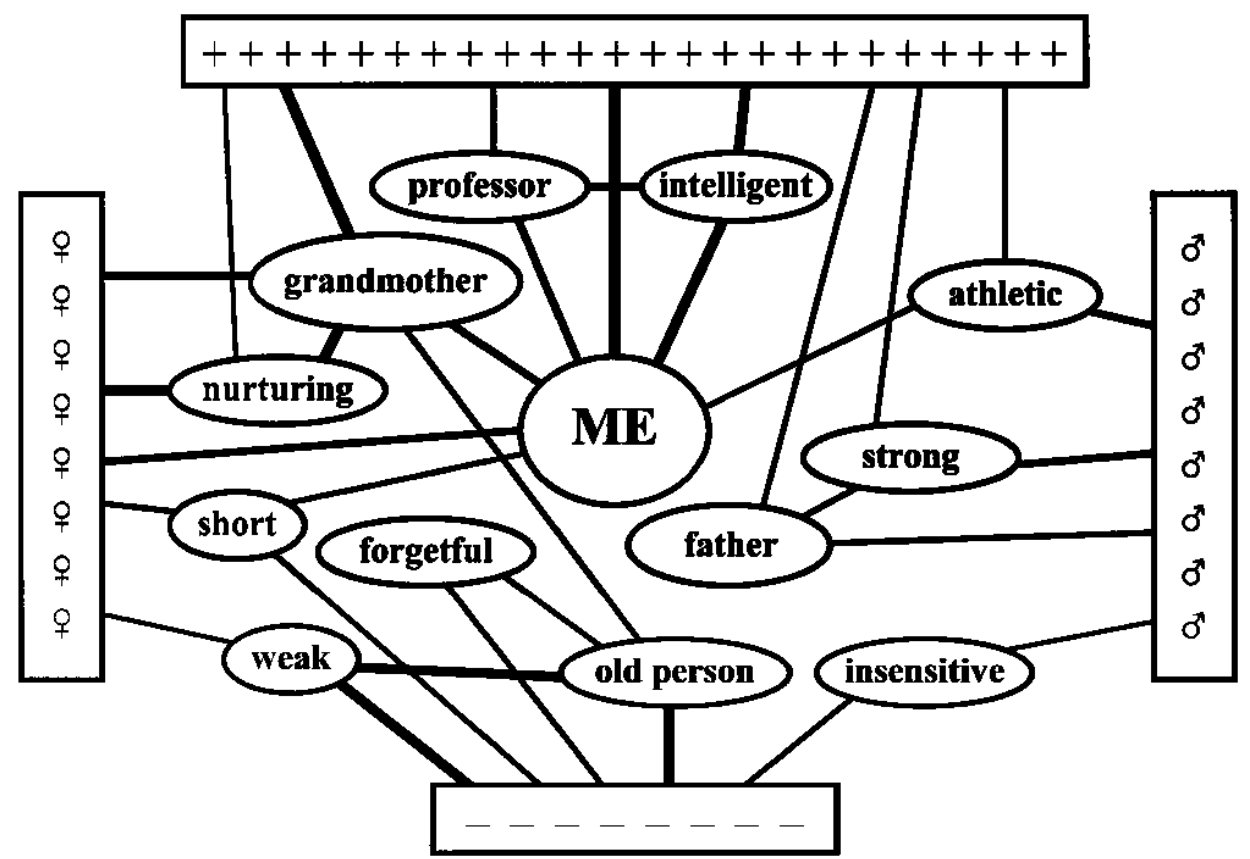

Figure 14. A social knowledge structure depicting the self-concept as a collection of associations (connections) between the "ME" node and traits (e.g., "intelligent"). Copied from Greenwald et al. (2002) for illustrative purposes.

Although the united theory does not specify how memories are retrieved, it does specify memory structure and associations. Let us consider the self-reference task and how engaging in that task may activate concepts within this structure. When making a self-reference judgment concerning a specific trait adjective, say intelligent, there would be activation of the "ME" and "Intelligent" nodes in the semantic network, and those nodes would activate other linked concepts (e.g., "intelligent" would activate "professor") to varying degrees (i.e., the thickness of the lines represent strength of association). After making a self-reference decision, concepts that were activated during the process may constitute episodic memory traces that comprise the encoded itemspecific information. For example, the activated concept "professor" may be one memory trace that could serve as an item-specific cue for the adjective intelligent. Relational information in this structure can be represented as general category nodes that are 
common to multiple nodes that were activated during the task (e.g., "ME", "positive valence", or perhaps "openness", which is not shown).

When asked to recall the adjectives that were encoded, the task instructions and encoded relational information may be used to define a search set (Hunt \& McDaniel, 1993). Thus, participants might ask themselves: "Which words were related to me and which ones were not?”, "Which words were positive and which ones were negative?", "Which words were related to openness and which ones were not?", and so on. The whole collection of episodic memory traces relevant to the considered relational cues would vary in size across participants. That is, participants who had more activated associations in their self-concept when making self-reference decisions would have more memory traces, hence a larger search set (hypothesized to be people who are high on a trait that corresponds to the self-referenced adjectives). When searching memory, a relational cue would constrain the search set to the traces relevant to the relational cue, and switching to a different relational cue at some point may constrain the search set to another collection of relevant traces. In this way, relational information can be effective if it reduces the search set size being considered at a given point in time.

Wixted and Rohrer's (1994) review of studies examining cumulative recall showed that search set size is negatively associated with the rate of approach to asymptote, with a larger search set producing slower recall. Basic random search models of memory predict this finding (e.g., McGill, 1963). Specifically, assuming random sampling with replacement from a finite search set, successful retrieval of list items based on sampled memory traces is most probable at the beginning of the recall task. As recall proceeds with traces being randomly sampled and replaced, however, there become fewer 
traces in the search set that will elicit retrieval of a new item (i.e., one that has not already been recalled). This property of the model explains the exponential slow down of recall (Equation 1) as search set size increases. When the search set is larger because some target adjectives have multiple traces that can produce successful retrieval (e.g., if many associations of the target adjective were activated during self-reference), recall would be slower because the probability of randomly selecting a trace that would elicit retrieval of a different adjective would be smaller. The pattern of cumulative recall in Study 1 is consistent with this scenario, wherein recall for highly orderly participants was slower but overall amount recalled was equivalent to their counterparts. In contrast, when the search set size is larger because more of the presented adjectives had associated memory traces that could lead to successful retrieval (e.g., more trait adjectives activated other concepts during self-reference), recall would not only be slower but would eventually elicit a higher asymptotic level (assuming enough time is given for recall). The pattern of cumulative recall as a function of openness in Study 2 is consistent with this scenario.

The social knowledge structure of Greenwald et al.'s (2002) theory could be reasoned about the same way to provide an account for the slower recall in the otherreference task of Study 3 (i.e., by assuming spread of activation of concepts associated with a best friend and then random sampling with replacement from item-specific, episodic memory traces). While the discussion around how a memory structure like that proposed by Greenwald et al. (2002) illustrates one way to look at the results of the present studies, the results of their studies suggested that the associations in the semantic network were activated automatically, such that indirect measures like the IAT captured the strength of associations within it, but not corresponding direct measures. 


\section{Is item-specific information in the personality self-concept propositional or}

\section{associative?}

An exploratory objective of the present research was to examine whether the additional item-specific information elicited by the self-reference task among those high on a corresponding trait is akin to the propositions of the explicit personality self-concept or the associations of the implicit personality self-concept (Schnabel \& Asendorpf, 2010; Gawronski \& Bodenhausen, 2011). Study 1 found that self-reference recall speed of the orderliness adjectives was negatively associated with the direct measures of conscientiousness, orderliness, and encoding-orderliness ( $r \mathrm{~s}=-.22$ to -.26$)$, but was not significantly associated with the indirect measure (i.e., orderliness-IAT; $r=-.12$ ). ${ }^{27}$

Similarly, Study 2 found that recall speed of the self-referenced openness adjectives was negatively related to the direct measure of openness $(r=-.24)$, but was not related to an indirect measure (i.e., openness-IAT; $r=-.03) .{ }^{28}$ In Study 3, recall speed of the selfreferenced openness adjectives was not related to either the direct or indirect measures of openness. In sum, the results indicate that the item-specific information that slowed down

\footnotetext{
${ }^{27}$ It is noteworthy that the orderliness-IAT in Study 1 did in fact seem to capture implicit orderliness, in that it correlated with the direct personality measures (conscientiousness, $r=.19$; orderliness, $r=.16$; encoding-orderliness, $r=.22$ ) with similar magnitude as that reported in previous research. Specifically, Grumm and Colani's (2007) conscientiousness-IAT was positively correlated with a direct measure of conscientiousness (NEO-FFI-30; $r=.18$ ) and another direct measure of the trait adjectives used in the IAT $(r=.24)$. Similarly, Schmukle, Back, and Egloff's (2008) conscientiousness-IAT was positively correlated with ratings of the adjectives used in the IAT $(r=.26)$ and with the NEO-FFI $(r=.22)$. Lastly, Steffens and Schulze-König (2006) reported $r=.22$ between their conscientiousness-IAT and directly measured conscientiousness by the NEO-FFI.

${ }^{28}$ It is also noteworthy that the openness-IAT in Study 2 and 3 generally correlated with the direct personality measures with a similar magnitude as has been reported in previous research (Study 2: openness, $r=-.01$; imaginativeness, $r=-.04$; encoding-openness, $r=-.17$; Study 3: openness, $r=.03$; imaginativeness, $r=.07$; encoding-openness, $r=.20$ ). Specifically, Grumm and Colani's (2007) opennessIAT correlated with a direct measure of openness (NEO-FFI-30; $r=.21$ ) and another direct measure obtained from ratings of the adjectives used in the IAT $(r=-.04)$. Similarly, Schmukle, Back, and Egloff (2008) reported weak correlations between their openness-IAT and ratings of the adjectives used in the IAT $(r=.04)$ and the NEO-FFI $(r=.09)$. Lastly, Steffens and Schulze-König (2006) reported $r=.02$ between their openness-IAT and NEO-FFI openness.
} 
recall was akin to the propositions of the explicit personality self-concept, not the strength of the associations that gave rise to the propositions. However, in contrast to the excellent psychometric properties of the direct personality measures that were used, it is possible that the lower level of reliability of the personality-IATs, and presumably the recall speed measure, reduced the likelihood of finding a consistent relationship between the IAT and recall speed across studies. Notwithstanding that issue, the present research tentatively suggests that the associative-propositional evaluation model proposed by Gawronski and Bodenhausen $(2006,2011)$ may be a better account of the present data than Greenwald et al.'s (2002) united theory, in that it may be the propositions that were elicited by the activation of associations in the self-concept that served as the itemspecific information searched for in memory.

It may seem counterintuitive that more activated item-specific information in a propositional (or associative) format would slow down recall, and may not even produce higher levels of recall. Let us turn to an early theory of recall that provides a way to think about how this could be.

\section{When more is less: cue-overload theory and recall speed}

Several memory paradigms have shown that free recall performance is impaired when events become associated with more retrieval cues. Watkins and Watkins (1976) proposed cue-overload theory to explain a growing list of such phenomena at that time. The theory suggests that recall performance of some episodic event is a function of the number of retrieval cues associated with it. One example of a phenomenon that the theory offers an explanation for is the impaired recall in the part list-cuing paradigm, wherein categorized word lists are presented as to-be-remembered material. In Watkins (1975), 
participants listened to the categorized words read aloud and were later asked to free recall the words. They were provided a booklet with a series of pages, and each page had a relevant category name printed at the top, with either zero, three, or six words that were heard earlier (intra-list cues). Recall probability was highest when the category label was present with zero cues, lower when presented with three cues, and lowest when presented with six cues. Cue-overload theory suggested that re-presentation of the list items at recall created additional redundant memory traces that were relevant for recall (i.e., they were associated with the category that served as relational information) thus reducing the probability of recalling other list items (Slamecka, 1968; Roediger, 1974).

Watkins (1975) found that this cueing effect on recall held even when the cues were not part of the memory list, but instead were new category instances (extra-list cues). The fact that extra-list cues also impaired recall probability was difficult to account for by the alternative explanation that presentation of the cues at recall strengthened itemcategory associations in memory (i.e., because extra-list cues were not presented earlier, they should not strengthen the particular item-category associations that were formed). Instead, the idea that new memory traces related to the label were formed and reduced the probability of recall of the other list items was a better fit with the results. In support of that idea, Rodiger, Stellon, and Tulving (1977; Experiment 1) compared the time course of free recall of 48 unrelated nouns in conditions of 0 (i.e., standard free recall), 16 intralist, or 32 intra-list cues. Participants were given 10 minutes for recall. Roediger et al. found that recall of the 16 critical items (i.e., ones that did not serve as recall cues in any condition) was slower in the 32 intra-list cue condition than in the other two conditions (recall was nominally slower in the 16 intra-list cue condition relative to free recall). A 
second experiment examined the impact of both intra- and extra-list cues on cumulative recall performance in five conditions: free recall; 32 intra-list cues (same as first experiment); 32 intra-list cues (studied for one minute just prior to recall); 32 extra-list cues (which were categorically unrelated to the list material), and 16 intra- and 16 extralist cues. Consistent with cue-overload theory, recall of a critical set of items was highest in the free recall condition, significantly reduced in the 32 intra-list (studied for one minute) and 32 extra-list cue conditions, and reduced further in the 32 intra-list (same as experiment 1) and 16 intra- and 16 extra-list cue conditions. Furthermore, recall of these critical items was significantly slower in all conditions that included intra-list cues relative to free recall and the 32 extra-list cue condition. In sum, the results concerning recall speed and amount recalled were consistent with cue-overload theory, which suggested that when relevant cues were presented at recall, or when studied for one minute and then taken away before recall, additional memory traces increased the search set size which slowed down recall and in these cases lead to fewer recalled items even after a 10 minute recall period (see also Mueller \& Watkins, 1977).

The cue-overload theory also offers an explanation for other effects on free recall. The list length effect, for example, shows that the probability of recalling any given item on a list is lower when the item is embedded in a longer list than in a shorter list. In this case, cue-overload theory suggests that the additional memory traces acquired when processing a longer list lowers the probability of recall of any given item on the list. Another example is the build-up of proactive interference, wherein a series of studyrecall trials (e.g., in one study-recall trial, participants would be shown 3 items and then tested on free recall) of new but related words on each list renders decreasing free recall 
performance across successive trials (e.g., Watkins \& Watkins, 1975). A release from this proactive interference can be obtained by presenting items from a new category in a subsequent study-recall trial. Cue-overload theory suggests that the number of relevant memory traces increases across the successive study-recall trials (i.e., search set size increases), reducing the probability of retrieval of list items shown on later trials relative to earlier ones, thus creating the proactive interference. When new category members are presented in a subsequent study-recall trial, there is a release from pro-active interference (i.e., recall probability rebounds to initial levels) because the memory traces from the preceding study-recall trials that caused the interference are no longer relevant (i.e., the relational cue guiding memory retrieval would now be the new category label, not the old one). All these examples are consistent with the idea that an increase in search set size reduces performance (any one memory trace is less likely to be sampled and lead to successful retrieval).

Wixted and Rohrer's (1994) review of studies examining cumulative recall curves concluded that a basic random search model of memory offers a simple explanation for the exponential slow down of free recall over time. In particular, the random search model suggests that there is an inverse relationship between search set size and asymptotic recall. That is, the larger the defined search set of memory traces, the slower recall proceeds over time, which is in accord with cue-overload theory The importance of search set size must be stressed, in that it is the search set size not the total amount recalled that is related to recall speed. In support of that idea, research has shown that total amount recalled has a negative, positive, or null relationship with recall speed under various conditions (Burns \& Schoff, 1998; Wixted \& Rohrer, 1994). Thus, based on this 
model, recall speed is a measure of search set size, and arguably of elaborative processing, that is independent from amount recalled and it offers insight into the breadth of search in long-term memory during recall.

What is important for the present research is whether the encoding of more itemspecific information creates a larger search set size. Burns and Schoff (1998) suggested that the results of their experiments were not consistent with recall speed being a good proxy for search set size, in that they found rate differences between conditions that were assumed to have an equal size search set (i.e., the number of encoded items in each condition was the same). However, it is possible that the encoding of more item-specific information is not all in one memory trace, but instead, may be a collection of traces. Intuitively, it may be that several propositions come to mind when deciding whether a trait adjective is self-descriptive. For example, when presented with the task of deciding whether the adjective conscientious is self-descriptive, activated associations in the selfconcept may give rise to generic propositions such as "sometimes I am late", "I work all the time", "people say I am conscientious", and so on. If these generic propositions are captured as separate episodic memory traces that can elicit retrieval of the adjective conscientious, then the build-up of item-specific information in this case could be detrimental to recall performance (i.e., a cue-overload), in that now there are multiple traces to retrieve the same list item, effectively increasing search set size without increasing the number of items that can be retrieved (and slowing down recall).

This situation is consistent with the cumulative recall performance of Burns and Schoff (1998; Experiment 1 and 2; see Figure 1), in which a list of items from ad hoc categories were either sorted (relational processing) or multiple item-specific processing 
tasks were conducted on the items. Recall was much slower for the item-specific processing group, which presumably processed a lot of item-specific information and did not have efficient relational information to narrow the search set (because neither the ad hoc category items nor the encoding task would have naturally encouraged relational processing). It took approximately 15 minutes for the item-specific processing group to reach nominally equivalent recall as the relational processing group. A cue-overload interpretation suggests that several memory traces were acquired when engaging in the multiple item-specific processing tasks, which slowed down recall considerably for this group, whereas the relational processing group encoded fewer item-specific cues (only those naturally encoded when sorting the unique ad hoc category items) and would have been able to constrain the search of memory using the relational information acquired during the sorting task (i.e., the ad hoc categories labels; e.g., Bower, 1970; Bower, Clark, Lesgold, \& Winzenz, 1969; Raajimakers \& Shiffrin, 1981).

The cue-overload idea is also consistent with the cumulative recall performance of Study 1 (see Figure 3), but under a different combination of item-specific and relational processing. Given that the materials afforded minimal relational information (i.e., all adjectives were related to conscientiousness), it is assumed that any relational information that would have been acquired by the task (e.g., "words that described me vs. those that did not") or by the general category binding the material (e.g., "words that were related to conscientiousness") would be inefficient to subdivide the acquired memory traces by a relevant category for more efficient search. In the context of ineffective relational information, additional item-specific memory traces encoded by the high orderliness group would have slowed down recall initially (i.e., cue-overload) but 
eventually would have caught up to the medium and low groups, who were hypothesized to have encoded less item-specific information (i.e., a smaller search set). Further research examining whether encoding more item-specific information from materials does in fact create multiple memory traces is warranted, but the idea is consistent with the data considered here and the predictions of a random search model of memory retrieval (Wixted \& Rohrer, 1994; Raajimakers \& Shiffrin, 1981; McGill, 1963).

One interesting avenue of approach to test that idea would be to use the part-list cuing procedure described earlier with the self-reference task of the present studies. In particular, participants could be presented a number of adjectives from the self-reference task as cues during recall (e.g., zero, three, or six cues). If it is true that self-referencing trait adjectives that are personality congruent renders more item-specific information in the form of multiple memory traces, one would expect participants who are low on the trait and are presented three or six cues at recall to have similar recall speed as participants who are high on the trait and presented zero cues at recall. The logic being that the latter group would have formed multiple traces during the self-reference task, whereas the former group would gain additional relevant traces during cued recall. Furthermore, it would be expected that the fastest recall of all conditions would be among those who are low on the trait and presented zero cues at recall, whereas and the slowest recall of all conditions would be among participants who are high on the trait and presented three or six cues at recall.

\section{Limitations and strengths}

The present studies have notable limitations and strengths. One limitation was that cumulative recall curves could not be adequately fit to participants' recall of adjectives 
encoded in the semantic and shallow tasks because amount recalled was too low and a high proportion of participants did not recall any of these adjectives. Thus, it was not possible to test a second set of research hypotheses concerning divergent validity of the recall speed measure. Specifically, it was expected that recall speed of the adjectives encoded in the self-reference task would be related to the target trait, but that recall of the adjectives in the semantic and shallow tasks would not be related to the target trait. In an effort to test this extra dissociation, recall curves were fit to group-level (low, medium, and high on the target trait) recall performance for the combined structural, rhyming, and semantic tasks in Study 1 and Study 2. The estimated rate of approach to asymptote showed that the negative personality-recall speed association observed in the selfreference task of Study 1 and Study 2 did not occur in the combined encoding conditions (i.e., high orderliness was not related to slower recall speed of the combined encoding tasks in Study 1 nor was high openness related to slower recall speed of the combined encoding tasks in Study 2). While these fits supported divergent validity by confirming the hypothesized negative personality-recall speed association was isolated to the selfreference task, the poor recall in the combined encoding conditions made the conclusions drawn from these analyses tentative.

A related limitation was the use of a within-subjects design in Study 3. Although the design was a good choice in that it allowed for a test of uncrossed double dissociation between the target and non-target traits of the self- and other-reference tasks within the same sample, the data and previous research (e.g., Burns et al., 2011; Burns et al., 2013) suggested that asking participants to recall the adjectives encoded in the two tasks at the same time obscured the recall speed measures, which limited tests of convergent, 
divergent, and predictive validity. Further research using either a between-subjects design or providing separate recall trials for the self- and other-referenced adjectives could help determine whether the failure to replicate was due to the design.

Participants in the present studies had not quite reached asymptotic recall by the end of the 10 minute (Study 1 and 2) or 14 minute (Study 3) recall period. Unfortunately, this aspect of the experimental procedure may have weakened measurement of recall speed somewhat by not allowing for additional items to be recalled beyond the recall period among those who had not exhausted item-specific cues (especially in Study 3). For example, Roediger and Thorpe (1978; see also Roediger, Payne, \& Gillespie, 1982) showed that in certain conditions recall persists well beyond 10 minutes, especially if many items have been encoded and there are high levels of item-specific processing. It should be noted, however, that in these studies many more words were presented in each encoding task (e.g., 20 words per task in Experiment 1) than in the present studies (i.e., 10 or 13 trait adjectives per task), which would extend the time required to reach asymptotic recall (all other things being equal; Wixted \& Rohrer, 1994). Furthermore, the plots of cumulative recall in the present studies suggest that participants were not far from asymptotic recall by the end of the 10 minutes provided in Study 1 and 2 (e.g., see Figure 3 and Figure 5 respectively), however the 14 minutes may not have been long enough in Study 3 (see Figure 9). Thus, although the use of a longer recall period in future research could be profitable, it is unlikely that the recall test length negatively impacted the main results of the present studies.

Another potential limitation worth discussing is that the present studies did not manipulate the adjective list structure (i.e., a related vs. an unrelated list). As was 
reviewed in the Introduction, an important factor to consider when examining cumulative recall is whether the items would naturally encourage acquisition of relational or itemspecific information. The work of Einstein and Hunt (1980; Hunt \& Einstein, 1981) showed that encoding tasks that encourage item-specific processing (e.g., a semantic task) benefit more in terms of recall from a list of items that belong to several distinct categories (i.e., a related list), whereas encoding tasks that encourage relational processing (e.g., category sorting) benefit more from a list of unique items that do not share an obvious category structure (i.e., an unrelated list). Klein and Loftus (1988) showed that the self-reference effect was eliminated when the list structure and encoding task together encouraged acquisition of relational and item-specific information. Hence, self-referencing was of benefit to memory because it provided both relational and itemspecific information (but list structure per se may not have affected self-reference recall, because the task may provide relatively redundant information, but may still increase search set size if differences among items are noticed).

With that in mind, the reported self-reference effect in the present studies was likely exaggerated. Consider the trait conscientiousness adjectives from Study 1, particularly those assigned to the semantic and self-reference tasks (i.e., the controlled and orderliness lists, respectively). These trait adjectives did not differ in latent semantic association to the impulsivity and orderliness concepts, and likely would not have elicited any relational information other than that the items generally described conscientiousness. Moreover, the list certainly did not promote acquisition of itemspecific information either (i.e., all the items were highly related, thus differences between them would likely not be naturally encoded). Hence, the list used in this study 
was atypical, in that it did not provide useful item-specific or relational information. The self-reference effect was therefore likely exaggerated by the atypical list structure (i.e., lists used in other studies have encouraged processing of one type of information, which would serve to boost recall more in the semantic task than in the self-reference task). Indeed, recall of the semantic adjectives in Study 1 was half that reported by Rogers et al. (1977), who selected adjectives from a wide range of trait categories, likely providing some useful relational information to narrow the search of memory. In contrast, in Study 2, the orderly and imaginative lists applied to the self-reference and semantic tasks were categorically distinct, acting more like a related list with only two categories. Recall of the semantically encoded items doubled in this study relative to Study 1, suggesting participants noticed the categorical separation of the lists and used that as a relational cue in the search of memory. Consistent with that idea, self-reference recall was also faster in Study 2, suggesting relational information helped speed-up recall. It should be noted that the categorical relation between the lists was likely somewhat redundant with the encoding task manipulation. That is, participants could have organized retrieval by encoding task (adjectives that were judged for meaning vs. self-referenced) or by trait categories (adjectives describing conscientiousness vs. openness).

Indeed, the materials used in the present studies were carefully selected to limit the relational and item-specific information that they may naturally elicit. It was important to ensure that the self-referenced adjectives (and the other-referenced ones) were all associated with one trait category, as the goal was to test whether participants who were high on the target trait category would recall personality congruent adjectives more slowly. It may in fact be beneficial to limit such information because it is known 
that item-specific and relational information afforded by the materials affects recall speed (Burns \& Hebert, 2005), thus creating construct-irrelevant variance for the personalityrecall speed relationships of interest in the present studies. Although list structure was indeed an important consideration, manipulating it was not pivotal to reach the goals of the present studies, but could be an interesting avenue to pursue in future research. For example, if openness is truly related to encoding more relational information, open participants should achieve higher asymptotic recall when encoding an unrelated list of items relative to their less open counterparts, but that should not happen when encoding a related list of items that naturally affords effective relational information.

Although there were key a priori predictions in the present studies, there were also a vast number of additional exploratory analyses. For example, the pursuit of establishing predictive validity of the recall speed and other memory-based measures involved a "kitchen sink" approach that included many indicators of trait-related behaviour. The statistical approach (i.e., zero-order correlations) did not limit the number of tests that were required. Given the vast number of inferential tests that were conducted, it must be acknowledged that the family-wise error rate for many of the sets of analyses was much higher than the standard rate of $\alpha=.05$. A related limitation was that the size of the observed convergent validity coefficients between recall speed and the direct personality measures in Study 1 and 2 were fairly small (i.e., $r=-.22$ to -.26). However, it must be noted that these coefficients are of similar magnitude to the population correlation between self-reports and corresponding IATs $(\rho=.24$; observed in Hofmann et al.'s, 2005 meta-analysis). Moreover, the correlations were likely subjected to considerable method variance (Cote \& Buckley, 1987; e.g., direct trait measures were captured using 
five-point rating scales of dispositional phrases and yes or no responses to trait adjectives, while indirect trait measures were captured using response times from opposing conditions in the IAT as well as recall speed). Further research would benefit from employing structural equation modeling, not only to limit the number of inferential tests, but also to account for method and error variance (Podsakoff, MacKenzie, Lee \& Podsakoff, 2003).

A particular strength of the present studies was the novel approach taken to connect research on the self-reference effect in memory (Rogers et al., 1977; Symons \& Johnson, 1997), measurement of personality self-concept (Schnabel \& Asendorpf, 2010), the item-specific relational framework (Hunt \& McDaniel, 1993), and random search models of memory retrieval (Wixted \& Rohrer, 1994). The predicted item-specific difference in recall speed among those high on a trait is the type of evidence that can more concretely link self-reference memory and elaborative processing. Specifically, prior attempts have inferred elaboration based on amount recalled (which creates a circular cause-and-effect relationship), except for one study conducted by Klein et al. (1994; Experiment 1) that failed to find a significant item gain difference (and item gain is still a problematic measure of elaborative processing; Burns \& Hebert, 2005). Linking personality self-concept to item-specific processing in a self-reference task also helps to link a memory structure to the cognitive elaboration that has been repeatedly advocated for as a causal factor in producing the memory advantage, since Rogers et al.'s (1977) seminal publication.

The unified theory of Greenwald et al. (2002) and Gawronski and Bodenhausen's (2011) associative-propositional evaluation model were examples that highlight how 
there is potential for connecting influential social-cognitive theories with random search models of memory retrieval to better understand individual differences in the itemspecific and relational information afforded by the self-concept (or person memory in general). One strength of the present thesis is that it uses dimensions of the Big Five personality traits as a proxy for individual differences in the structure of personality selfconcept. Linking this structure to cumulative recall performance in carefully controlled self-reference memory experiments moves the literature forward by specifying a priori predictions about how the structure should affect cumulative recall curves under certain conditions. The selection of individual differences in personality self-concept was a natural choice, as traits have been shown to be stable and relatively enduring with high test-retest reliability and well-established construct validity, and the majority of studies on the self-reference effect have used trait adjectives as materials.

Another strength of the present thesis was that the results suggested that recall speed of self-referenced trait adjectives that bear on one trait holds potential as a novel trait measurement tool. The convergent and divergent validity implied by the uncrossed double dissociation established preliminary construct validity. More validation studies, however, are required to determine the conditions that elicit a stronger personality-recall speed relationship, some of which have already been discussed. The results also suggest that the personality measure can be applied to other people, as was observed in the otherreference task, tapping the participants' concept of their best friend's orderliness. This finding also suggests that the self does not have special properties in the sense that it engages qualitatively different processes or structure. It may be that self-referencing facilitates item-specific and relational processing because the memory structure that it 
taps happens to be more complex (i.e., more item-specific and relational information inherently available in the structure). As research has shown, circumstances can be produced wherein the list structure and encoding task naturally elicit the same level of processing as the self-reference task (as in Klein \& Loftus, 1988; see also research on the survival processing effect; Burns et al., 2011; Klein, 2012b). Nevertheless, the present research shows that memory structure is indeed an important factor to consider more closely, as was suggested by Lockheart and Craik (1990) and Einstein and Hunt (1980).

The level of experimental control over the materials is a notable strength of the present studies. The stimuli were selected from studies on the lexical approach to personality measurement and indirect personality measures, while taking into account the latent semantic association of the adjectives to the target concepts. Furthermore, the adjective lists applied to the semantic, self-reference, and other-reference tasks were equated for word frequency, word length, likeableness, and meaningfulness, and color words were used to buffer against primary and recency effects on memory. Thus, these confounding variables were likely not responsible for the observed differences in memory performance between lists.

Lastly, collection of the direct personality measures in mass testing in Study 2 was a notable strength. This change in procedure reduced the impact of demand characteristics on the observed relationship between directly measured personality and memory performance, which cast doubt on whether the main personality-recall speed results observed in Study 1 were due to demand characteristics. More generally, the change in procedure demonstrated that the negative openness-recall speed association held when the measures were separated in time and space. 


\section{Conclusions}

I argued that previous attempts to connect the elaborative processing involved in the self-reference effect to personality dimensions have been unsuccessful (e.g., Sullivan, 1998), or have lead to tenuous conclusions (e.g., Katz, 1989; see also Jones \& Brunell, 2014), due to a focus on the total amount recalled at the end of a relatively brief recall test (i.e., relative to the number of encoded items). The present studies took a novel approach comparing individual differences in personality traits to the full time course of free recall and to the item gains and losses on a second recall task. Recall performance across the studies supported the elaborative and organizational processing account of the self- and other-reference effects. More importantly, by focusing the stimulus materials on specific personality traits, a hypothesized and novel result surfaced, wherein slower recall was observed when personality congruent adjectives were self-referenced, suggesting that these participants engaged in more elaborate processing (i.e., encoded more item-specific information). It was suggested that the additional item-specific information might have rendered separate memory traces, which is consistent with an increase in the search set size known to slow down recall and with cue-overload theory. The negative personalityrecall speed association showed convergent and divergent validity in an uncrossed double dissociation between the target and non-target traits (orderliness and openness) in Study 1 and 2. Measures of item loss on the second recall trial suggested that the personalityrecall speed associations were not due to relational processing differences. Study 3 extended the phenomenon to the other-reference task, indicating that the association was not due to a special property of the self, but instead was due to a quantitative difference in the item-specific information elicited by a memory structure in a given encoding task. 
The within-subjects design of Study 3 was likely responsible for: the failed replication of the openness-recall speed association of Study 2, the relatively weakened personalityrecall speed association observed in the other-reference task, and the relatively poor predictive validity in Study 3. The item-specific information that slowed down recall appeared to be due to memory traces of propositions that were elicited by activation of associations in memory (i.e., a self-report measure), as opposed to being due directly to the strength of raw associations in memory (i.e., an IAT). Overall, the convergent and predictive validity coefficients were small, however that may have been due to substantial method variance that was not accounted for, poor reliability of the indirect measures (IAT and recall speed), and the use of a within-subjects design in the memory task. Nevertheless, the present studies offered a significant contribution in that they successfully linked a memory structure that is central to the self to the elaborative processing that occurs during self-reference using a principled analysis of cumulative recall that does not render a circular cause-and-effect relationship. Further work to explore this relationship within the item-specific relational framework, and the cueoverload interpretation of it, may bring a new angle to research on the self-reference effect and a potential novel trait measurement tool. 


\section{References}

Allport, G. W., \& Odbert, H. S. (1936). Trait-names: A psycho-lexical study. Psychological Monographs, 47 (1, Whole No. 211).

Anderson, N. H. (1968). Likeableness ratings of 555 personality trait words. Journal of Personality and Social Psychology, 9, 272-279.

Aron, A., Aron, E. N., \& Smollan, D. (1992). Inclusion of Other in the Self Scale and the structure of interpersonal closeness. Journal of personality and social psychology, $63,596$.

Asendorpf, J. B., Banse, R., \& Mucke, D. (2002). Double dissociation between implicit and explicit personality self-concept: The case of shy behavior. Journal of Personality and Social Psychology, 83, 380-393. doi:10.1037/0022-3514.83.2.380

Ashton, M. C. (1998). Personality and job performance: the importance of narrow traits. Journal of Organizational Behavior, 19, 289-303.

Aviv, A. L., Zelenski, J. M., Rallo, L. \& Larsen, R. J. (2002). Who comes when: Personality differences in early and later participation in a university subject pool. Personality and Individual Differences, 33, 487-496.

Back, M. D., Schmukle, S. C., \& Egloff, B. (2006). Who is late and who is early? Big Five personality factors and punctuality in attending psychological experiments. Journal of Research in Personality, 40, 841-848. doi:10.1016/j.jrp.2005.11.003

Back, M. D., Schmukle, S. C., \& Egloff, B. (2009). Predicting actual behavior from the explicit and implicit self-concept of personality. Journal of Personality and Social Psychology, 97, 533-548. doi:10.1037/a0016229 
Baddeley, A. D. (1978). The trouble with levels: A reexamination of Craik and Lockheart's framework for memory research. Psychological Review, 85, 139-152.

Banse, R., Seise, J., \& Zerbes, N. (2001). Implicit attitudes towards homosexuality: Reliability, validity, and controllability of the IAT. Zeitschrift fur Experimentelle Psychologie, 48, 145-160.

Bates, M. E., \& Lemay, E. P. (2004). The d2 test of attention: Construct validity and extensions in scoring techniques. Journal of the International Neuropsychological Society, 10, 392-400. doi: 10.1017/S135561770410307X

Block, J. (2005). A contrarian view of the five-factor approach to personality description. Psychological Bulletin, 117, 187-215.

Block, J. (2010). The five-factor framing of personality and beyond: Some ruminations. Psychological Inquiry, 21, 2-25. doi: 10.1080/10478401003596626

Bousfield, W. A., \& Sedgewick, C. H. W. (1944). An analysis of restricted associative responses. Journal of General Psychology, 30, 149-165.

Bousfield, A. K., \& Bousfield, W. A. (1966). Measurement of clustering and of sequential constancies in repeated free recall. Psychological Reports, 19, 935-942.

Bower, G. H. (1970). Organizational factors in memory. Cognitive Psychology, 1, 18-46.

Bower, G. H., Clark, M. C., Lesgold, A. M., \& Winzenz, D. (1969). Hierarchical retrieval schemes in recall of categorized word lists. Journal of Verbal Learning and Verbal Behavior, 8, 323-343.

Bower, G. H. \& Gilligan, S. G. (1979). Remembering information related to one's self. Journal of Research in Personality, 13, 420-432. 
Bradely, B., Mogg, K., Galbraith, M., \& Perrett, A. (1993). Negative recall bias and neuroticism: state vs trait effects. Behavior Research Therapy, 31, 125-127. doi: $0005-7967 / 93$

Brickencamp, R. (1981). Aufmerksamkeits-Belastungs-Test d2 [d2 Test of Attention]. Göttingen: Hogrefe.

Brickenkamp, R., \& Zillmer, E. (1998). The d2 test of attention. Hogrefe \& Huber Pub.

Brysbaert, M., \& New, B. (2009). Moving beyond Kucera and Francis: A critical evaluation of current word frequency norms and the introduction of a new and improved word frequency measure for American English. Behavior Research Methods, 41, 977-990.

Burns, D. J. (1989). Proactive interference: An individual-item versus relational processing account. Journal of Memory and Language, 28, 345-359.

Burns, D. J. (1993). Item gains and losses during hypermnesic recall: Implications for the item-specific-relational distinction. Journal of Experimental Psychology: Learning, Memory, and Cognition, 19, 163-173.

Burns, D. J., Jenkins, C. I., \& Dean, E. E. (2007). Falsely recalled items are rich in itemspecific information. Memory \& Cognition, 35, 1630-1640.

Burns, D. J., Martens, N. J., Bertoni, A. A., Sweeney, E. J., \& Lividini, M. D. (2006). An item gains and losses analysis of false memories suggest critical items receive more item-specific processing than list items. Journal of Experimental Psychology: Learning, Memory, \& Cognition, 32, 277-289. 
Burns, D. J., \& Hebert, T. (2005). Using cumulative-recall curves to assess the extent of relational and item-specific processing, Memory, 13, 189-199. doi:10.1080/09608210344000670

Burns, D. J., Hwang, A. J., \& Burns, S. A. (2011). Adaptive memory: Determining the proximate mechanism responsible for the memorial advantages of survival processing. Journal of Experimental Psychology: Learning, Memory, \& Cognition, 37, 206-218. doi: 10.1037/a0021325

Burns, D. J., \& Schoff, K. M. (1998). Slow and steady often ties the race: Effects of itemspecific and relational processing on cumulative recall. Journal of Experimental Psychology: Learning, Memory, \& Cognition, 24, 1041-1051.

Chan, J. C., \& McDermott, K. B. (2007). The testing effect in recognition memory: A dual process account. Journal of Experimental Psychology: Learning, Memory, and Cognition, 33, 431-437.

Collins, A. M., \& Quillian, M. R. (1969). Retrieval time from semantic memory. Journal of verbal learning and verbal behavior, 8, 240-247.

Conway, A. R. A., Kane, M. J., Bunting, M. F., Hambrick, D. Z., Wilhelm, O., \& Engle, R. W. (2005). Working memory span tasks: A methodological review and user's guide. Psychonomic Bulletin \& Review, 12, 769-786.

Costa, P. T., Jr., \& McCrae, R. R. (1992). Revised NEO Personality Inventory (NEO-PIR) and NEO Five-Factor Inventory (NEO-FFI) professional manual. Odessa, FL: Psychological Assessment Resources.

Cote, J. A., \& Buckley, M. R. (1987). Estimating trait, method, and error variance: Generalizing across 70 construct validation studies. Journal of Marketing 
Research, 24, 315-318.

Craik, F. I. M., Moroz, T. M., Moscovitch, M., Stuss, D. T., Winocur, G., Tulving, E., Kapur, S. (1999). In search of the self: A positron emission tomography study. Psychological Science, 10, 26-34.

Craik, F. I. M., \& Lockheart, R. S. (1972). Levels of processing: A framework for memory research. Journal of Verbal Learning and Verbal Behavior, 11, 671-684.

Craik, F. I. M., \& Tulving, E. (1975). Depth of processing and the retention of words in episodic memory. Journal of Experimental Psychology: General, 11, 268-294.

Crowne, D. P., \& Marlowe, D. (1960). New scale of social desirability independent of psychopathology. Journal of Consulting Psychology, 24, 349-354.

De Raad, B., \& Peabody, D. (2005). Cross-culturally recurrent personality factors: Analyses of three factors. European Journal of Personality, 19, 451-474. doi: $10.1002 /$ per. 550

De Houwer, J. (2006). What are implicit measures and why are we using them? In R. Wiers \& a. Stacy (Eds.), Handbook of implicit cognition and addiction (pp. 1128). New York: Sage.

Dunn, J. C., \& Kirsner, K. (1988). Discovering functionally independent mental processes: the principle of reversed association. Psychological review, 95, 91-101.

Ebersole, C. R., Atherton, O. E., Belanger, A. L., Skulborstad, H. M., Adams, R. B., Allen, J., ... Nosek, B. A. (2015). Many Labs 3: Evaluating participant pool quality across the academic semester via replication. Retrieved from osf.io/ct89g

Egloff, B., \& Schmukle, S. C. (2002). Predictive validity of an implicit association test for assessing anxiety. Journal of Personality and Social Psychology, 83, 1441- 
1455. doi:10.1037/0022-3514.83.6.1441

Einstein, G. O., \& Hunt, R. R. (1980). Levels of processing and organization: Additive effects of individual items and relational processing. Journal of Experimental Psychology: Human Learning and Memory, 6, 588-598.

Eysenck, M. W. (1978). Levels of processing: A critique. British Journal of Psychology, 68, 157-169.

Figueredo, A. J., Sefcek, J. A., \& Jones, D. N. (2006). The ideal romantic partner personality. Personality and Individual Differences, 41, 431-441.

Gawronski, B. (2002). What does the Implicit Association Test measure? A test of the convergent and discriminant validity of prejudice-related IATs. Experimental Psychology, 49, 171-180. doi: 10.1027//1618-3169.49.3.171

Gawronski, B., \& Bodenhausen, G. V. (2006). Associative and propositional processes in evaluation: An integrative review of implicit and explicit attitude change. Psychological Bulletin, 132, 692-731.

Gawronski, B., \& Bodenhausen, G. V. (2011). The associative-propositional evaluation model: Theory, evidence, and open questions. In M. P. Zanna (Ed.), Advances in Experimental Social Psychology (pp. 59-127) doi: 10.1016/B978-0-12-385522$0.00002-0$

Glazner, M., \& Cunitz, A. R. (1966). Two storage mechanisms in free recall. Journal of Verbal Learning and Verbal Behavior, 5, 351-360.

Goldberg, L. R. (1990). An alternative "description of personality": The Big-Five Factor structure. Journal of Personality and Social Psychology, 59, 1216-1229.

Goldberg, L. R., Johnson, J. A., Eber, H. W., Hogan, R., Ashton, M. C., Cloninger, C. R., 
\& Gough, H. G. (2006). The international personality item pool and the future of public-domain personality measures. Journal of Research in Personality, 40, 8496. doi: 10.1016/j.jrp.2005.08.007

Greenwald, A. G., Banaji, M. R., Rudman, L. A., Farnham, S. D., Nosek, B. A., \& Mellott, D. S. (2002). A unified theory of implicit attitudes, stereotypes, selfesteem, and self-concept. Psychological review, 109, 3.

Greenwald, A. G., McGhee, D. E., \& Schwartz, J. L. K. (1998). Measuring individual differences in implicit cognition: The implicit association test. Journal of Personality and Social Psychology, 74, 1464-1480.

Greenwald, A. G., Nosek, B. A., \& Banaji , M. R. (2003). Understanding and using the implicit association test: I. An improved scoring algorithm. Journal of Personality and Social Psychology, 85, 179-216. doi: 10.1037/0022-3514.85.2.197

Grumm, M., \& Collani, G. (2007). Measuring Big-Five personality dimensions with the implicit association test - Implicit personality or self-esteem? Personality and Individual Differences, 43, 2205-2217. doi: 10.1016/j.paid.2007.06.032

Halpin, J. A., Puff, C. R. Mason, H. F., \& Martson, S. P. (1984). Self-reference and incidental recall by children. Bulletin of the Psychonomic Society, 22, 87-89.

Harris, J. A. (2004). Measured intelligence, achievement, openness to experience, and creativity. Personality and Individual Differences, 36, 913-929.

Hege, A. C., \& Dodson, C. S. (2004). Why distinctive information reduces false memories: evidence for both impoverished relational-encoding and distinctiveness heuristic accounts. Journal of Experimental Psychology: Learning, Memory, and Cognition, 30, 787. Retrieved from: 
http://digitalcommons.butler.edu/cgi/viewcontent.cgi?article=1181\&context=facs ch_papers

Hofmann, W., Gawronski, B., Gschwendner, T., Le, H., \& Schmitt, M. (2005). A metaanalysis on the correlation between the Implicit Association Test and explicit selfreport measures. Personality and Social Psychology Bulletin, 31, 1369-1385.

Hunt, R. R., \& Einstein, G. O. (1981). Relational and item-specific information in memory, Journal of Verbal Learning and Verbal Behavior, 20, 497-514.

Hunt, R. R., \& McDaniel, M. A. (1993). The enigma of organization and distinctiveness. Journal of Memory and Language, 32, 421-445.

Hunt, R. R., Elliot, M., \& Spence, M. J. (1979). Independent effects of process and structure on encoding. Journal of Experimental Psychology: Human Learning, and Memory, 5, 339-347.

Hyde, T. S., \& Jenkins, J. J. (1969). Differential effects of incidental tasks on the organization of recall of a list of highly associated words. Journal of Experimental Psychology, 82, 472-491.

Jackson, D. N. (1976). Jackson personality inventory manual. Goshen, NY: Research Psychologists Press.

Jackson, D. N. (1984). Multidimensional aptitude battery manual. Port Huron, MI: Research Psychologists Press.

Jackson, D. N. (1989). Personality research form manual (3rd ed.). Port Huron, MI: Sigma Assessment Systems.

Jacoby, L. L. (1991). A process dissociation framework: Separating automatic from intentional uses of memory. Journal of memory and language, 30, 513-541. 
James, W. (1890). Principles of psychology (Vol. 1) New York, NY: Henry Holt and Company.

Johnson, J. A., \& Ostendorf, F. (1993). Clarification of the five-factor model with the abridged Big Five dimension circumplex. Journal of Personality and Social Psychology, 65, 563-576.

Jones, L. L., \& Brunell, A. B. (2014). Clever and crude but not kind: Narcissism, selfesteem, and the self-reference effect. Memory, 22, 307-322.

Katz, A. N. (1987). Self-reference in the encoding of creative-relevant traits. Journal of Personality, 55, 97-120.

Kelly, W. M., Macrea, C. N., Wyland, C. L., Caglar, S. Inati, S., \& Heatherton, T. F. (2002). Finding the self? An event-related fMRI study. Journal of Cognitive Neuroscience, 15, 785-794.

Kircher, T. T. J., Senior, C., Phillips, M. L., Benson, P. J., Bullmore, E., Brammer, M., Simmons, A., Williams, S. C. R., Bartels, M., David, A. S. (2000). Towards a functional neuroanatomy of self processing: effects of faces and words. Cognitive Brain Research, 10, 133-144.

Klein, S. B. (2012a). Self, memory, and the self-reference effect: An examination of conceptual and methodological issues. Personality and Social Psychology Review, 16, 283-300. doi: 10.1177/1088868311434214

Klein, S. B. (2012b). A role for self-referential processing in tasks requiring participants to imagine survival on the savannah. Journal of Experimental Psychology: Learning, Memory, and Cognition, 38, 1234.

Klein, S. B., \& Kihlstrom, J. F. (1986). Elaboration, organization, and the self-reference 
effect in memory. Journal of Experimental Psychology: General, 115, 26-38.

Klein, S. B., \& Loftus, J. (1988). The nature of self-referent encoding: The contributions of elaborative and organizational processes. Journal of Personality and Social Psychology, 55, 5-11.

Klein, S. B., Loftus, J., \& Burton, H. A. (1989). Two self-reference effects: The importance of distinguishing between self-descriptiveness judgments and autobiographical retrieval in self-referent encoding. Journal of Personality and Social Psychology, 56, 853.

Klein, S. B., Loftus, J., Kihlstrom, J. F. \& Aseron, R. (1989). Effects of item-specific and relational information on hypermnesic recall. Journal of Experimental Psychology: Learning, Memory, and Cognition, 15, 1192-1197.

Klein, S. B., Loftus, J., \& Schell, T. (1994). Repeated testing: A technique for assessing the roles of elaborative and organizational processing in the representation of social knowledge. Journal of Personality and Social Psychology, 66, 830-839.

Klein, S. B., Loftus, J., \& Sherman, J. W. (1993). The role of summary and specific behavioral memories in trait judgments about the self. Personality and Social Psychology Bulletin, 19, 305-305.

Klein, S. B., Loftus, J., Trafton, J. G., \& Fuhrman, R. W. (1992). Use of exemplars and abstractions in trait judgments: A model of trait knowledge about the self and others. Journal of Personality and Social Psychology, 63, 739.

Landauer, T. K., Foltz, P. W., \& Laham, D. (1998). Introduction to latent semantic analysis. Discourse Processes, 25, 259-284.

Lee, K., \& Ashton, M. C. (2004). Psychometric properties of the HEXACO Personality 
Inventory. Multivariate Behavioral Research, 39, 329-358.

Lockheart, R. S., \& Craik, F. I. M. (1990). Levels of processing: A retrospective commentary on a framework for memory research. Canadian Journal of Psychology, 44, 87-112.

Mandler, G. (1967). Organization and memory. In K. W. Spence \& J. T. Spence (Eds.), The psychology of learning and motivation, Vol.1 New York: Academic Press.

Markus, H. (1977). Self-schemata and processing information about the self. Journal of Personality and Social Psychology, 35, 63-78.

McCrea, R. R., \& Costa, P.T. (1987). Validation of the five-factor model of personality across instruments and observers, Journal of Personality and Social Psychology, $52,81-90$.

McCrea, R. R., \& Costa, P.T. (1997). Personality trait structure as a human universal, American Psychologist, 52, 509-516.

McCrea, R. R., \& Sutin, A. R. (2009). Openness to Experience. In M. R. Leary \& R. H. Hoyle (Eds.), Handbook of individual differences in social behaviour (pp. 257 273).

McCabe, D. P., Presmanes, A. G., Robertson, C. L., \& Smith, A. D. (2004). Item-specific processing reduces false memories. Psychonomic Bulletin \& Review, 11, 10741079 .

McGill, W. J. (1963). Stochastic latency mechanisms. In D. Luce (ed.), Handbook of Mathematical Psychology. John Wiley \& Sons.

Mueller, C. W., \& Watkins, M. J. (1977). Inhibition from part-set cuing: A cue-overload interpretation. Journal of Verbal Learning and Verbal Behavior, 16, 699-709. 
Norman, W. T. (1963). Toward an adequate taxonomy of personality attributes: Replicated factor structure in peer nomination personality ratings. Journal of Abnormal and Social Psychology, 66, 574-583.

Norman, W. T. (1967). 2800 personality trait descriptors: Normative operating characteristics for a university population. Ann Arbor: University of Michigan, Department of Psychology.

Paulhus, D. L. (1984). Two-component models of socially desirable responding. Journal of Personality and Social Psychology, 46, 598-609.

Paunonen, S. V. (1998). Hierarchical organization of personality and prediction of behavior. Journal of Personality and Social Psychology, 74, 538-556.

Paunonen, S. V. (2003). Big Five factors of personality and replicated prediction. Journal of Personality and Social Psychology, 84, 411-424. doi: 10.1037/00223514.84 .2 .411

Paunonen, S. V., \& Ashton, M. C. (2001a). Big Five factors and facets and the prediction of behavior. Journal of Personality and Social Psychology, 81, 524-539.

Paunonen, S. V., \& Ashton, M. C. (2001b). Big Five predictors of academic achievement. Journal of Research in Personality, 35, 78-90.

Peters, K. R., \& Gawronski, B. (2011). Mutual influences between the implicit and explicit self-concepts: The role of memory activation and motivated reasoning. Journal of Experimental Social Psychology, 47, 436-442.

Podsakoff, P. M., MacKenzie, S. B., Lee, J. Y., \& Podsakoff, N. P. (2003). Common method biases in behavioral research: a critical review of the literature and recommended remedies. Journal of applied psychology, 88, 879. 
Raajimakers, J. W, \& Shiffrin, R. M. (1981). Search of associative memory. Psychological Review, 88, 93-134.

Rasmussen, A. S., \& Berntsen, D. (2010). Personality traits and autobiographical memory: Openness is positively related to the experience and usage of recollections. Memory, 18, 774-786.

Roediger, H. L. (1974). Inhibiting effects of recall. Memory \& Cognition, 2, 261-269.

Roediger, H. L., Payne, D. G., Gillespie, G. L., \& Lean, D. S. (1982). Hypermnesia as determined by level of recall. Journal of Verbal Learning and Verbal Behavior, $21,635-655$.

Roediger, H. L., Stellon, C. C., \& Tulving, E. (1977). Inhibition from part-list cues and rate of recall. Journal of Experimental Psychology: Human Learning and Memory, 3, 174.

Roediger, H. L., \& Thorpe, L. A. (1978). The role of recall time in producing hypermnesia. Memory and Cognition, 6, 286-305.

Roenker, H. L., Thompson, C. P., \& Brown, S. C. (1971). Comparison of measures for the estimation of clustering in free recall. Psychological Bulletin, 76, 45-48.

Rogers, T. B. (1977). Self-reference in memory: Recognition of personality items. Journal of Research in Personality, 11, 295-305.

Rogers, T. B., Kuiper, N. A., \& Kirker, W. S. (1977). Self-reference and the encoding of personal information. Journal of Personality and Social Psychology, 35, 677-688.

Rogers, T. B., Rogers, P. J., \& Kuiper, N. A. (1979). Evidence for the self as a cognitive prototype: The "False alarm effect". Personality and Social Psychology Bulletin, 5, 53-56. doi: 10.1177/014616727900500111 
Rohrer, D., \& Wixted, J. T. (1994). An analysis of latency and interresponse time in free recall. Memory \& Cognition, 22, 511-524.

Rusting, C. L., \& Larsen, R. J. (1998). Personality and cognitive processing of affective information. Personality and Social Psychology Bulletin, 24, 200-213. doi: $10.1177 / 0146167298242008$

Schnabel, K., \& Asendorpf, J. B. (2010). The self-concept: New insights from implicit measurement procedures. In B. Gawronski \& B. K. Payne (Eds.). Handbook of Implicit Social Cognition: Measurement, Theory, and Applications (pp. 408-425). New York: Guilford Press.

Schmukle, S. C., Back, M. D., \& Egloff, B. (2008). Validity of the Five-Factor Model for the implicit self-concept of personality. European Journal of Psychological Assessment, 24, 263-272. doi: 10.1027/1015-5759.24.4.263

Slamecka, N. J. (1968). An examination of trace storage in free recall. Journal of Experimental Psychology, 76, 504-513.

Steffens, M. C. (2004). Is the implicit association test immune to faking? Experimental Psychology, 51, 165-179. doi: 10.1027/1618-3169.51.3.165

Steffens, M. C., \& Schulze-König, S. (2006). Predicting spontaneous Big Five behavior with implicit association tests. European Journal of Psychological Assessment, 22, 13-20. doi: 10.1027/1015-5759.22.1.13

Strack, F., \& Deutsch, R. (2004). Reflective and impulsive determinants of social behavior. Personality and Social Psychology Review, 8, 220-247.

Sullivan, M. (1998). The five-factor model and the processing of self-relevant information (Doctoral dissertation). Retrieved from 
http://digitool.library.mcgill.ca/R/?func=dbin-jump-

full\&object_id=35631\&local_base=GEN01

Symons, C. S., \& Johnson, B. T. (1997). The self-reference effect in memory: A metaanalysis. Psychological Bulletin, 121, 371-394. Retrieved from http://digitalcommons.uconn.edu/chip_docs/9/

Tabachnick, B. G., \& Fidell, L. S. (2007). Using Multivariate Statistics (5th ed.). Boston: Pearson Education.

Trapnell, P. D., \& Wiggins, J. S. (1990). Extension of the Interpersonal Adjective Scales to include Big Five dimensions of personality. Journal of Personality and Social Psychology, 59, 781-790.

Tulving, E. (1962). Subjective organization in free recall of "unrelated" words. Psychological Review, 69, 344-354.

Tulving, E. (1966). Subjective organization and effects of repetition in multi-trial freerecall learning. Journal of Verbal Learning and Verbal Behavior, 5, 193-197.

Tupes, E. C., \& Christal, R. E. (1961). Recurrent personality factors based on trait ratings (USAF ASD Tech. Rep. No. 61-97). Lackland Air Force Base, TX: U.S. Air Force.

Unsworth, N., \& Engle, R. W. (2007). The nature of individual differences in working memory capacity: Active maintenance in primary memory and controlled search from secondary memory. Psychological Review, 114, 104-132. doi: 10.1037/0033-295X.114.1.104

Unsworth, N., Redick, T. S., Heitz, R. P., Broadway, J. M., \& Engle, R. W. (2009). Complex working memory span tasks and higher-order cognition: A latent- 
variable analysis of the relationship between processing and storage. Memory, 17, 635-654. doi: 10.1080/09658210902998047

Watkins, M. J. (1975). Inhibition in recall with extralist “cues”. Journal of Verbal Learning and Verbal Behavior, 14, 294-303.

Watkins, M. J., \& Watkins, O. C. (1976). Cue-overload theory and the method of interpolated attributes. Bulletin of the Psychonomic Society, 7(3), 289-291.

Watkins, O. C., \& Watkins, M. J. (1975). Buildup of proactive inhibition as a cueoverload effect. Journal of Experimental Psychology: Human Learning and Memory, 1, 442.

Witt, E. A., Donnellan, M. B., \& Orlando, M. J. (2011). Timing and selection ef- fects within a psychology subject pool: Personality and sex matter. Personality and Individual Differences, 50, 355-359.

Wixted, J. T., \& Rohrer, D. (1993). Proactive interference and the dynamics of free recall. Journal of Experimental Psychology: Learning, Memory, \& Cognition,19, 1024-1039.

Wixted, J. T., \& Rohrer, D. (1994). Analyzing the dynamics of free recall: An integrative review of the empirical literature. Psychonomic Bulletin \& Review, 1, 89-106.

Young, O. C. D., \& Martin, M. (1981). Processing of information about self by neurotics. British Journal of Clinical Psychology, 20, 205-212.

Zelenski, J. M. (2007). Experimental approaches to individual differences and change: Exploring the causes and consequences of extraversion. In A. D. Ong and M. Van Dulman (Eds.), Oxford Handbook of Methods in Positive Psychology (pp. 205219). Oxford University Press. 
Zelenski, J. M., Rusting, C. L., \& Larsen, R. J. (2003). Consistency in the time of experiment participation and personality correlates: A methodological note. Personality and Individual Differences, 34, 547-558.

Zelenski, J. M., Santoro, M. S., \& Whelan, D. C. (2012). Would introverts be better off if they acted more like extraverts? Exploring emotional and cognitive consequences of counter-dispositional behavior. Emotion, 12, 290-303. doi: 10.1037/a0025169

Zhiyan, T., \& Singer, J. L. (1996). Daydreaming styles, emotionality and the Big Five personality dimensions. Imagination, Cognition, and Personality, 16, 399-414. 


\section{Appendices}

\section{Appendix A: Procedure for Creating the Levels-of-Processing Lists}

All conscientiousness adjectives from De Raad and Peabody's (2005) impulse control and orderliness facets were examined. The two other facets (responsibleness and work) were excluded because of the low number of adjectives describing those facets, particularly on the negative poles. There were 18 adjectives for the positive pole of impulse control and 25 for the negative pole, and 22 adjectives for the positive pole of orderliness and 25 for the negative pole. Word length and word frequency (measured in $\log 10$ [raw frequency count +1 ] units) were then computed following Brysbaert and New (2009) on the Ghent University website (http://expsy.ugent.be/subtlexus/ and http://subtlexus.lexique.org/moteur2/). All adjectives with a relatively low frequency (less than $1.50 \log 10$ units) were excluded, leaving a pool of 49 adjectives to select from, with over 10 adjectives for the four poles of the two facets.

To acquire four lists of exactly 10 adjectives, two considerations were made.

First, a decision was made to eliminate terms in one list that were simply the negated version of a term in another list (e.g., organized and disorganized), in an effort to eliminate alternative processing strategies in the recall task (e.g., organizational processing of memory list items by the root word) and the IAT (e.g., adopting alternative categorization strategies based on more negated words for one concept relative to another; Schmukle, Back, \& Egloff, 2008). When one term had been used in a previous conscientiousness-IAT (e.g., organized, Steffens, 2004; Steffens \& Schulze-König, 2006), the corresponding negated term was excluded. Otherwise, the decision to exclude one and not the other was made based on trying to equate lists in terms of word length 
and word frequency. Second, using a many-to-one latent semantic analysis tool on the University of Colorado website (http://lsa.colorado.edu/) with a topic space of "General Reading up to 1 st year college (300 factors)", the latent semantic associations of the impulsive, impulse control, impulsivity, order, orderly, and orderliness concepts were computed for all 49 adjectives. The average association for each adjective with its corresponding concepts (e.g., cautious with impulsive, impulse control, and impulsivity) was computed, as was the average association for the other concepts (e.g. cautious with order, orderly, and orderliness). The final criterion for including adjectives was to maximize the distance between the orderliness and impulse control concepts, by excluding those with a higher association with an opposing concept (e.g., cautious having a higher association to orderliness than impulse control), while checking word frequency when facing a decision between two items with similar associations to the two concepts.

Eight items were selected to buffer against the primacy and recency effects on memory. When selecting these items, it was deemed most important to choose adjectives that were unrelated to the adjectives in the memory lists and were also from the same category. Word frequency and word length were not deemed important, as these words were simply ignored when examining recall. As such, color words were chosen because they were largely unrelated to the items in the lists and were highly categorically related in an LSA analysis. In particular, the primary color words (red, green, blue), secondary color words (cyan, magenta, yellow) as well as two arbitrarily chosen color words (orange and brown) were selected. These eight words were highly related to each other $(M=.47, S D=.23)$, but largely unrelated to the items in the controlled $(M=.065, S D=$ $.056)$, orderly $(M=.044, S D=.042)$, impulsive $(M=.053, S D=.067)$, and disorderly $(M$ 
$=.052, S D=.050)$ lists. The words occur highly frequently in the English language $(M=$ 2.93, $S D=1.07)$ and are relatively short in length $(M=5.00, S D=1.31)$. 


\section{Appendix B: Rhyming and Semantic Orienting Questions}

The words selected to replace XXXX in the rhyming orienting questions ("Rhymes with XXXX?") were collected from RhymeZone (http://www.rhymezone.com/). Rhymes were considered appropriate when they were categorically unrelated to the memory list and recognition items. It was important to select these stimuli from diverse categories to ensure that participants could not learn to press "No" when they see an exemplar from a specific category within the orienting question. For instance, if all orienting questions that required a "No" response were exemplars from the vegetable category, participants could accurately respond to the question without processing the trait adjective (i.e., simply by seeing an exemplar of the vegetable category in the orienting question).

The words selected to replace XXXX in the semantic orienting questions ("'Means the same as XXXX?") were collected from the Merriam Webster's on-line thesaurus (http://www.merriam-webster.com). In particular, "Yes" responses were from the lists of synonyms or near synonyms and the "No" responses were from the lists of antonyms or near antonyms. The "Yes" responses necessarily had to be adjectives that describe people because a synonym for a trait adjective must also be a trait adjective. The "No" responses were therefore also adjectives that described people, in order to ensure that participants would not learn that a "Yes" response is required when a trait adjective is presented in the orienting question and that a "No" response is required otherwise. Careful attention was paid to not replicate or use the root of one of the words in the memory or recognition lists. The stimuli selected for the rhyming and semantic orienting questions are displayed in Appendix H. 


\section{Appendix C: Reading Span Stimuli and Order of Presentation}

\begin{tabular}{|c|c|c|c|c|}
\hline Span & Sentence & Yes & No & Letter \\
\hline \multirow[t]{3}{*}{ P1 } & $\begin{array}{l}\text { Just because there's a XXXX on his finger } \\
\text { doesn't mean he's married }\end{array}$ & ring & lake & - \\
\hline & $\begin{array}{c}\text { Finding a whole XXXX in an apple is better } \\
\text { than finding half of one }\end{array}$ & worm & fad & - \\
\hline & $\begin{array}{l}\text { Anyone who dares open the XXXX shall } \\
\text { suffer the mummy's curse }\end{array}$ & tomb & nap & - \\
\hline \multirow[t]{3}{*}{$\mathrm{P} 2$} & $\begin{array}{c}\text { Make sure the pockets on your XXXX are big } \\
\text { enough for your wallet }\end{array}$ & jeans & list & $\mathrm{P}$ \\
\hline & $\begin{array}{l}\text { Before hunting in winter, hunters must cut } \\
\text { through the ice to place } \mathrm{XXXX}\end{array}$ & decoys & laundry & $\mathrm{Z}$ \\
\hline & $\begin{array}{c}\text { The lawnmower is in the XXXX with the } \\
\text { other gardening tools }\end{array}$ & shed & bed & A \\
\hline \multirow[t]{2}{*}{2} & $\begin{array}{l}\text { The boy's mother bought him new shoes at } \\
\text { the } X X X X\end{array}$ & shoe store & $\begin{array}{l}\text { bus } \\
\text { stop }\end{array}$ & V \\
\hline & $\begin{array}{l}\text { They were on the XXXX when they saw the } \\
\text { car crash }\end{array}$ & bus & exam & $\mathrm{S}$ \\
\hline \multirow[t]{3}{*}{3} & $\begin{array}{c}\text { I placed the XXXX in the newspaper last } \\
\text { Monday morning }\end{array}$ & $a d$ & wave & $\mathrm{D}$ \\
\hline & $\begin{array}{c}\text { As a prank, Terry's friends shaved his XXXX } \\
\text { while he was asleep }\end{array}$ & head & car & W \\
\hline & $\begin{array}{l}\text { The route was clear as soon as the XXXX was } \\
\text { unrolled }\end{array}$ & map & milk & I \\
\hline \multirow[t]{3}{*}{4} & $\begin{array}{l}\text { I will meet you at the food court in twenty } \\
\qquad \mathrm{XXXX} \text {. minutes }\end{array}$ & minutes & carrots & $\mathrm{O}$ \\
\hline & $\begin{array}{l}\text { To adjust the level up or down, turn the } \\
\text { XXXX marked 'Volume' }\end{array}$ & knob & grass & $\mathrm{H}$ \\
\hline & $\begin{array}{l}\text { The children had been asleep for hours, } \\
\text { snuggled comfortably in their XXXX }\end{array}$ & beds & forks & $\mathrm{J}$ \\
\hline
\end{tabular}




\begin{tabular}{|c|c|c|c|c|}
\hline & $\begin{array}{l}\text { The cookies were still out of reach, but the } \\
\qquad \text { XXXX was on the table }\end{array}$ & milk & floor & $\mathrm{T}$ \\
\hline \multirow[t]{5}{*}{5} & $\begin{array}{l}\text { Janice kicked the XXXX over the goalie's } \\
\text { head and into the net }\end{array}$ & ball & house & $X$ \\
\hline & $\begin{array}{l}\text { Like he had done every Christmas morning, } \\
\text { Johnny unwrapped every XXXX }\end{array}$ & present & coma & $\mathrm{R}$ \\
\hline & $\begin{array}{l}\text { It was hard to stay quiet with the jingling of } \\
\qquad \mathrm{XXXX} \text { in her pocket }\end{array}$ & coins & leaves & Q \\
\hline & $\begin{array}{l}\text { Through the window Barb thought she saw a } \\
\text { cloaked XXXX walking toward her }\end{array}$ & figure & pasta & $\mathrm{L}$ \\
\hline & $\begin{array}{l}\text { Reaching for the doorknob, he realized that } \\
\text { his XXXX were in his other coat }\end{array}$ & keys & cows & $\mathrm{C}$ \\
\hline \multirow[t]{6}{*}{6} & $\begin{array}{l}\text { A sign of a poorly maintained engine is dark } \\
\text { XXXX billowing from the tailpipe }\end{array}$ & exhaust & flowers & M \\
\hline & $\begin{array}{l}\text { Tourists are kindly asked to refrain from } \\
\text { taking } \mathrm{XXXX} \text { from the beaches }\end{array}$ & shells & fonts & $\mathrm{G}$ \\
\hline & $\begin{array}{l}\text { Since I was sick the day they assigned } \\
\text { instruments, I had to play french XXXX }\end{array}$ & horn & toast & $\mathrm{K}$ \\
\hline & $\begin{array}{l}\text { The XXXX was unable to stop before the } \\
\text { tracks ended, resulting in a major wreck }\end{array}$ & train & rose & I \\
\hline & $\begin{array}{l}\text { The border official will request to see your } \\
\text { XXXX upon entering the country }\end{array}$ & passport & autumn & $\mathrm{U}$ \\
\hline & $\begin{array}{c}\text { At some } \boldsymbol{X X X X} \text {, a potential employer may ask } \\
\text { you to list your weaknesses }\end{array}$ & interviews & lullabies & $\mathrm{F}$ \\
\hline \multirow[t]{3}{*}{7} & $\begin{array}{l}\text { MP3s were okay for some people, but she } \\
\text { preferred listening to XXXX on compact disc }\end{array}$ & music & rainbows & $\mathrm{O}$ \\
\hline & $\begin{array}{l}\text { Randy took postal work seriously, making } \\
\text { sure the XXXX was always on time }\end{array}$ & mail & beach & B \\
\hline & $\begin{array}{l}\text { Last Halloween, she carved a XXXX to look } \\
\text { like a ghost }\end{array}$ & pumpkin & faucet & W \\
\hline
\end{tabular}




\begin{tabular}{|r|r|r|c|}
\hline $\begin{array}{r}\text { Please keep all arms and legs inside the } \\
\text { XXXX at all times }\end{array}$ & vehicle & pharmaci \\
st & Y \\
\hline $\begin{array}{r}\text { The XXXX is where I get all of my meat and } \\
\text { fresh fruit }\end{array}$ & $\begin{array}{r}\text { farmer's } \\
\text { market }\end{array}$ & $\begin{array}{c}\text { fire } \\
\text { station }\end{array}$ & L \\
\hline $\begin{array}{r}\text { Looking down to check his XXXX, Craig } \\
\text { realized how long he'd been waiting } \\
\text { I spent the weekend sick in bed, watching TV, } \\
\text { and drinking hot XXXX }\end{array}$ & watch & branch & S \\
\hline
\end{tabular}

Note: Bold italics indicate the quasi-randomly selected stimuli presented within the sentence in place of XXXX. 


\section{Appendix D: Procedure for Creating Recognition Memory Lists}

For the recognition memory items, the 40 lures were the trait adjectives that remained after selecting the memory list items from the impulse control and orderliness facets of conscientiousness presented in De Raad and Peabody's (2005) study. To create the list, compound adjectives and hyphenated adjectives were first excluded because the memory list items did not contain either of those types of adjectives, and that difference would presumably facilitate correct rejections in the recognition memory test (i.e., participants could simply remember that hyphenated or compound adjectives were not presented in the levels-of-processing task). Furthermore, when adjectives shared the root of a word and only differed in a prefix such that one adjective was a negated version of the other (e.g., cautious and incautious), only one of the two was selected. Finally, adjectives that were not defined in the SUBTLEXus database were excluded (e.g., digressive, discontinuous, and thrifty). The remaining adjectives comprised the lures for each list, with 4 impulse control, 10 orderly, 8 impulsive, and 7 disorderly lures.

The remaining 11 lures were taken from the adjectives listed under the two other facets of conscientiousness in De Raad and Peabody's (2005) study. In particular, three lures were selected from the positive pole of responsibleness and three from the negative pole of work. Correspondingly, three were selected from the positive pole of work and the only two available adjectives from the negative pole of responsibleness were selected. In total, there were 20 lures from the positive pole of the facets of conscientiousness and 20 from the negative pole of those facets. Across the 80 memory list and lure items, there were no instances where one item was the negated version of another item.

Finally, to keep the categorically unrelated items consistent with the fact that all 
the memory list and lure adjectives describe something about people, adjectives that also describe people in other ways were selected. More precisely, a list of 38 adjectives that differed in whether they described height, weight, strength, or some combination of these was collected. Word frequency and word length were calculated. The 20 longest words in the list were selected to try and match the other stimuli on word length, but as the unrelated items were meant to be relatively obvious foils, word frequency and length were not considered important. Table D1 presents the categorically unrelated items, and at face value they appear to be distinct from the memory list and lure stimuli.

Table D1

Recognition Memory Lures and Categorically Unrelated Items

\begin{tabular}{|c|c|c|c|c|c|}
\hline \multicolumn{6}{|c|}{ Lures } \\
\hline $\begin{array}{l}\text { Impulse } \\
\text { Control }\end{array}$ & Orderly & Impulsive & Disorderly & $\begin{array}{c}\text { Responsible } \\
\text { ness }\end{array}$ & Work \\
\hline Circumspect & Thorough & Impetuous & Neglectful & Reliable & Diligent \\
\hline Provident & Systematic & Precipitous & Ineffective & Dependable & Industrious \\
\hline Frugal & Methodical & Carefree & Bungling & Dutiful & Productive \\
\hline \multirow[t]{7}{*}{ Parsimonious } & Businesslike & Nonchalant & Untidy & Irresponsible & Lazy \\
\hline & Painstaking & Unbridled & Inattentive & $\begin{array}{c}\text { Unconscienti } \\
\text { ous }\end{array}$ & Idle \\
\hline & Punctilious & Lustful & Inconsistent & & Sluggish \\
\hline & Fussy & Extravagant & Inconstant & & \\
\hline & Rigorous & Chattering & & & \\
\hline & Severe & & & & \\
\hline & Concise & & & & \\
\hline \multicolumn{6}{|c|}{ Categorically Unrelated } \\
\hline Slender & Stumpy & Brawny & Powerful & Skinny & \\
\hline Tallish & Petite & Stocky & Delicate & Gangly & \\
\hline
\end{tabular}




\begin{tabular}{clllc}
\hline Little & Robust & Small & Muscular & Giant \\
Strong & Stubby & Short & Towering & Scrawny \\
\hline
\end{tabular}

Note. Italicized items are on the negative pole of their corresponding constructs. 


\section{Appendix E: Customized Self-Paced d2 Test}

Although each trial (line) was not timed, the standard time limit for each line of 47 characters was reflected in the total time allowed for the test. That is, participants were given 20 seconds for each of the nine lines of 47 characters, rendering exactly a 3-minute test (the time required for a distracter task before free recall).

Each of the nine rows of 47 characters was generated in the following manner. To make the test fairly difficult, twice as many similar distracters (i.e., "d"s with one or three marks and "p"s with two marks) were assigned to each row than targets (i.e., "d"s with two marks). Specifically, each row consisted of 12 targets, 25 similar distracters, and 10 dissimilar distracters (i.e., "p"s with one or three marks). The 10 possible targets appeared once along with two randomly selected targets. From the 31 possible similar distracters, 25 were randomly selected without replacement. From the 21 possible dissimilar distracters, 10 were randomly selected without replacement. Within each row of 47 characters, the targets were randomly allocated to the possible serial positions. Then, the similar distracters were randomly allocated to the remaining positions, followed by the dissimilar distracters. Nine rows were created in this fashion. Unfortunately, the 9 rows could not be placed on the sheet of paper in a legible font. Therefore, they were placed in the order that they were generated within a 17 by 25 matrix (with only 23 characters on the $17^{\text {th }}$ row). Figure E1 displays the customized $d 2$ test. 


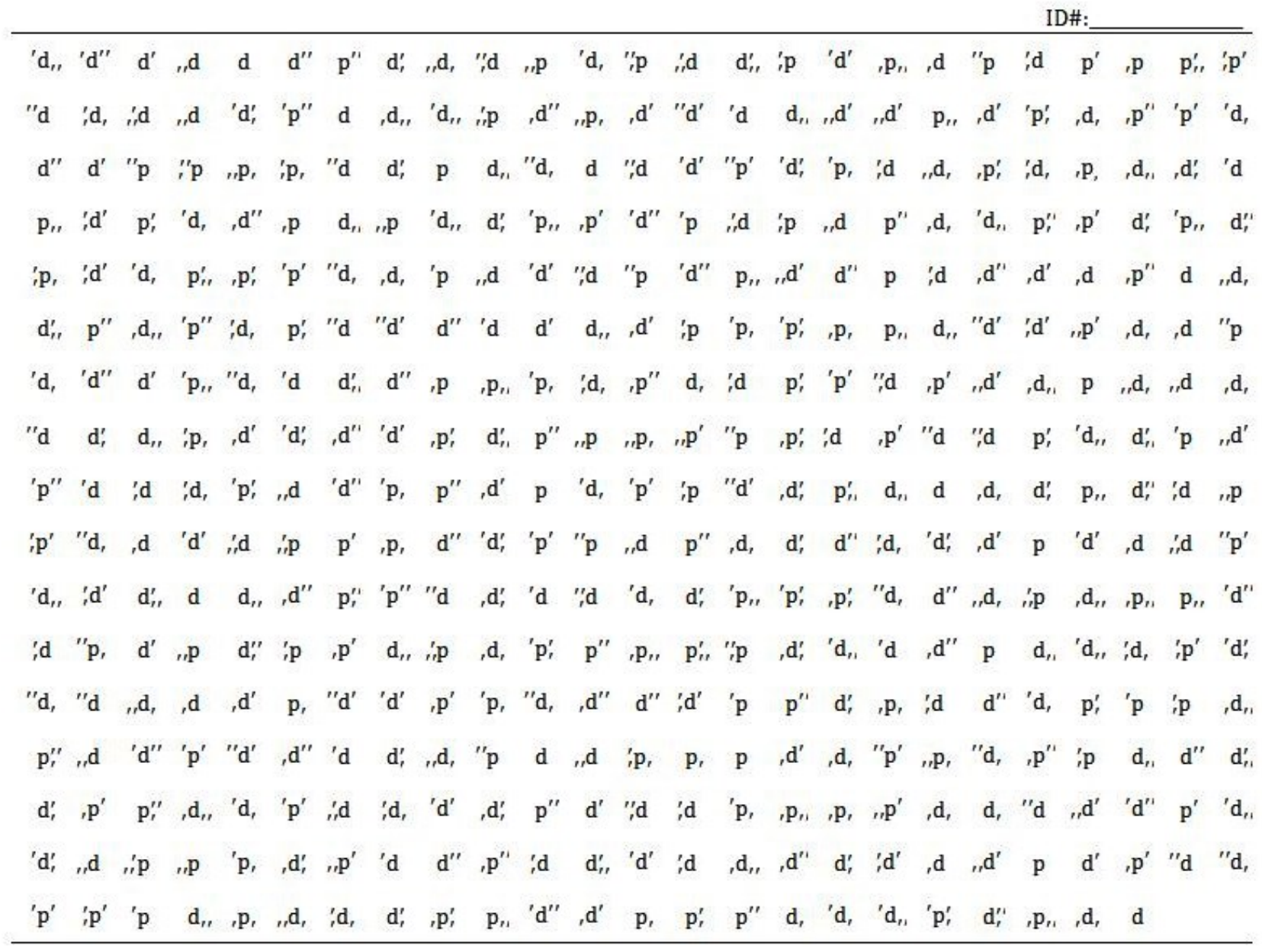

Figure E1. The customized self-paced d2 test. 


\section{Appendix F: Personality Questionnaire}

\section{Personality Questionnaire}

ID\#:

Date:

On the following pages, there are phrases describing different behavior. Please use the rating scale below to describe how accurately eachstatement describes you.

Describe yourself as you are nowin general, not as you wish to be in the future. Describe yourself as you honestly see yourself, in comparison to other people you know of the same sex and roughly the same age as you. Your responses will be kept in absolute confidence, so that you can describe yourself in an honest manner.

Please read each statement carefully, judge to what degree the statement applies to you, and write down the corresponding scale number in the space to the left of the statement number.

\begin{tabular}{|ccccc|}
\hline 1 & 2 & 3 & 4 & 5 \\
Very Inaccurate & $\begin{array}{c}\text { Moderately } \\
\text { Inaccurate }\end{array}$ & $\begin{array}{c}\text { Neither } \\
\text { Inaccurate } \\
\text { nor Accurate }\end{array}$ & $\begin{array}{c}\text { Moderately } \\
\text { Accurate }\end{array}$ & Very Accurate \\
\hline
\end{tabular}

1. Avoid philosophical discussions.

2. Have a vivid imagination

3. Carry out my plans.

4. Waste my time.

5. Leave a mess in my room.

6. Know how to captivate people.

7. Keep in the background.

8. Rarely get iritated.

9. Have frequent mood swings.

10. Dislike myself.

11. Tend to vote for conservative political candidates.

12. Feel comfortable aroundpeople.

13. Don't like to draw attention to myself.

14. Love order and regularity. 
ID\#:

\begin{tabular}{|ccccc|}
\hline 1 & 2 & 3 & 4 & 5 \\
Very Inaccurate & $\begin{array}{c}\text { Moderately } \\
\text { Inaccurate }\end{array}$ & $\begin{array}{c}\text { Neither } \\
\text { Inaccurate } \\
\text { nor Accurate }\end{array}$ & $\begin{array}{c}\text { Moderately } \\
\text { Accurate }\end{array}$ & Very Accurate \\
\hline
\end{tabular}

15. Go on binges.

16. Don't talk a lot.

17. Never spend more than I can afford

18. Am not bothered by disorder.

19. Believe in the importance of art.

20. Often feel blue.

21. Get chores done right away.

22. Carry the conversation to a higher level.

23. Do not enjoy going to art museums.

24. Shirk my duties.

25. Don't know why I do some of the things I do.

26. Do not like art.

27. Do things according to a plan.

28. Find it difficult to get down to work.

29. Am the life of the party.

30. Do just enough work to get by.

31. Have little to say.

32. Enjoy hearingnewideas.

33. Never splurge.

34. Would describe my experiences as somewhat dull.

35. Am not easily bothered by things.

36. Am skilled in handling social situations.

37. Am always prepared

38. Panic easily. 


\begin{tabular}{|ccccc|}
\hline 1 & & & & ID\#: \\
\hline Very Inaccurate & $\begin{array}{c}\text { Moderately } \\
\text { Inaccurate }\end{array}$ & $\begin{array}{c}\text { Neither } \\
\text { Inaccurate } \\
\text { nor Accurate }\end{array}$ & $\begin{array}{c}\text { Moderately } \\
\text { Accurate }\end{array}$ & Very Accurate \\
\hline
\end{tabular}

39. Am not bothered by messypeople.

40. Make plans andstick to them.

41. Pay attention to details.

42. Often eat too much.

43. Am able to controlmy cravings.

44. Rarely overindulge.

45. Leave my belongings around

46. Make friends easily.

47. Am not interested in abstractideas.

48. Seldom feel blue.

49. Am often down in the dumps.

40. Want everything to be "just right"

51. Love to eat.

52. Easily resist temptations.

53. Like order.

54. Don't see things through.

55. Often forget to put things back in their proper place.

56. Tend to vote for liberal political candidates.

57. Do things I later regret.

58. Feel comfortable with myself.

59. Like to tidy things up.

60. Am very pleased with myself. 


\section{Appendix G: Behaviour Report Questionnaire}

ID $\#$ :

\section{BR Questionnaire}

On the following pages, there are some questions asking your opinion on certain personal matters and others asking about personal facts. Some questions require a yes or no response, while others require you to use the provided rating scale or to provide a number.

For yes-no responses and the rating scales, please CIRCLE your selection. For the responses that require a number, please write the number in the space to the left of the question.

Please answer the questions in a way that accurately describes you right now. Remember, your responses will be kept in absolute confidence.

1. $\mathrm{M} \quad \mathrm{F}$ Yourgender?

2. Yes No Doyou enjoy reading classic literature?

3. Yes No Doyouplay any musicalinstroment(s)?

4. Yes No Doyouplay any organized sports?

5. Yes No Doyou exercise regularly (aerobics, weights, running, etc.)?

6. Yes No Is English your first language?

7a. Yes No Are you currently a fraternity/sorority member or pledge?

7b. If you are not currently a fraterrity/soronity member or pledge, how much interest would you have in joining a fratemity/soronity?

$\begin{array}{ccccccc}1 & 2 & 3 & 4 & 5 & 6 & 7 \\ \begin{array}{c}\text { Very } \\ \text { Uninterested }\end{array} & & & \text { Average } & & & \begin{array}{c}7 \text { Very } \\ \text { Interested }\end{array}\end{array}$




\section{ID\#:}

8. How would you rate your level of physical attractiveness compared to the average student?

$\begin{array}{ccccccc}1 & 2 & 3 & 4 & 5 & 6 & 7 \\ \begin{array}{c}\text { Very } \\ \text { Unattractive }\end{array} & & \text { Average } & & & \begin{array}{c}\text { Very } \\ \text { Attractive }\end{array}\end{array}$

9. How would you rate your level of general intelligence compared to the average student?

$\begin{array}{ccccccc}1 & 2 & 3 & 4 & 5 & 6 & 7 \\ \begin{array}{c}\text { Very } \\ \text { Unintelligent }\end{array} & & & \text { Average } & & & \begin{array}{c}\text { Very } \\ \text { Intelligent }\end{array}\end{array}$

10. In relation to people you know, how would you describe your popularity amongyour peers?

$\begin{array}{llcccccc}1 & 2 & 3 & 4 & 5 & 6 & 7 \\ \begin{array}{c}\text { Very } \\ \text { Unpopular }\end{array} & & & \text { Average } & & & \text { Very } \\ \text { Popular }\end{array}$

11. How would you describe your honesty compared to that of your friends?

$\begin{array}{ccccccc}1 & 2 & 3 & 4 & 5 & 6 & 7 \\ \begin{array}{c}\text { Very } \\ \text { Dishonest }\end{array} & & & \text { Average } & & & \begin{array}{c}\text { Very } \\ \text { Honest }\end{array}\end{array}$

12. Do you consider yourself (check one box):
ㅁ. Non-Smoker
$\square_{2}$ Infrequent Smoker
$\square_{3}$ Light Smoker
$\square_{4}$ Moderate Smoker
$\square_{5}$ Heavy Smoker 
ID \#:

13. Do you consider yourself (check one box):

ㅁ: Non-Drinker

$\square_{2}$ Infrequent Drinker

$\square_{3}$ Light Drinker

$\square_{4}$ Moderate Drinker

$\square_{5}$ Heavy Drinker

14. If you had to choose one program of study (or, if you have already chosen one), which of the two below would you (did you) choose? Please check one box.

$\square_{1}$ Liberal Arts (Arts, Fine Arts, Humanities, etc.)

$\square_{2}$ Non-liberal Arts (Natural Sciences, Social Sciences, etc.)

15. Indicate your overall average grade last year (as a $\%$ percentage).

16. Estimate your average number of dates per month that you

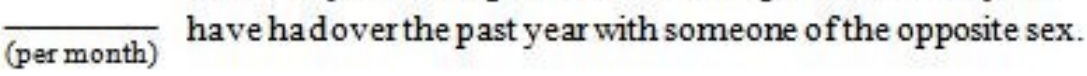

17. How many different people did you date over the course of the past year?

18. Please indicate your graduating average grade from $\mathrm{High}$ $\%$ School (as a percentage).

19. Please estimate the average number of parties per month that $\overline{(\text { per month) }}$ you attend.

20. How frequently do you clean your windows/mirrors at home (peryear) peryear? 


\section{Appendix H: Presentation Order for the Levels-of-Processing Task}

\begin{tabular}{|c|c|c|c|c|c|c|}
\hline \multirow[b]{2}{*}{ Trial } & \multirow[b]{2}{*}{ List } & \multirow[b]{2}{*}{ Stimuli } & \multicolumn{4}{|c|}{ Encoding Task (Orienting Question) } \\
\hline & & & $\begin{array}{l}\text { Structural } \\
\text { (Two or } \\
\text { more e's?) }\end{array}$ & $\begin{array}{c}\text { Rhyming } \\
\text { (Rhymes with } \\
\text { XXXX?) }\end{array}$ & $\begin{array}{c}\text { Semantic } \\
\text { (Means the same } \\
\text { as XXXX?) }\end{array}$ & $\begin{array}{c}\text { Self- } \\
\text { Reference } \\
\text { (Describes } \\
\text { me?) }\end{array}$ \\
\hline 1 & \multirow{4}{*}{ Color } & Green & Yes & & & \\
\hline 2 & & Cyan & & Began (yes) & & \\
\hline 3 & & Blue & & & Garlic (no) & \\
\hline 4 & & Magenta & & & & Yes or no \\
\hline 5 & \multirow{10}{*}{ Impulsive } & Impulsive & No & & & \\
\hline 6 & & Rash & No & & & \\
\hline 7 & & Reckless & Yes & & & \\
\hline 8 & & Hasty & No & & & \\
\hline 9 & & Frivolous & No & & & \\
\hline 10 & & Excessive & Yes & & & \\
\hline 11 & & Insatiable & No & & & \\
\hline 12 & & Restless & Yes & & & \\
\hline 13 & & Exaggerated & Yes & & & \\
\hline 14 & & Lavish & No & & & \\
\hline 15 & \multirow{10}{*}{ Disorderly } & Disorderly & & Quarterly (yes) & & \\
\hline 16 & & Negligent & & Prayerful (no) & & \\
\hline 17 & & Careless & & Student (no) & & \\
\hline 18 & & Lax & & Cracks (yes) & & \\
\hline 19 & & Confused & & Bruised (yes) & & \\
\hline 20 & & Chaotic & & Aquatic (yes) & & \\
\hline 21 & & Sloppy & & Bash (no) & & \\
\hline 22 & & Forgetful & & Delirious (no) & & \\
\hline 23 & & Contradictory & & Victory (yes) & & \\
\hline 24 & & Unpredictable & & Omniscient (no) & & \\
\hline 25 & \multirow{5}{*}{ Controlled } & Cautious & & & Bold (no) & \\
\hline 26 & & Careful & & & Random (no) & \\
\hline 27 & & Prudent & & & Wise (yes) & \\
\hline 28 & & Deliberate & & & Intentional (yes) & \\
\hline 29 & & Moderate & & & Reasonable (yes) & \\
\hline
\end{tabular}




\begin{tabular}{|c|c|c|c|c|c|c|}
\hline 30 & & Restrained & & & Flamboyant (no) & \\
\hline 31 & & Disciplined & & & Loose (no) & \\
\hline 32 & & Composed & & & Flustered (no) & \\
\hline 33 & & Serious & & & Earnest (yes) & \\
\hline 34 & & Practical & & & Pragmatic (yes) & \\
\hline 35 & \multirow{10}{*}{ Orderly } & Punctual & & & & Yes or no \\
\hline 36 & & Organized & & & & Yes or no \\
\hline 37 & & Efficient & & & & Yes or no \\
\hline 38 & & Particular & & & & Yes or no \\
\hline 39 & & Meticulous & & & & Yes or no \\
\hline 40 & & Strict & & & & Yes or no \\
\hline 41 & & Precise & & & & Yes or no \\
\hline 42 & & Accurate & & & & Yes or no \\
\hline 43 & & Measured & & & & Yes or no \\
\hline 44 & & Prompt & & & & Yes or no \\
\hline 45 & \multirow{4}{*}{ Color } & Red & no & & & \\
\hline 46 & & Brown & & Crown (yes) & & \\
\hline 47 & & Yellow & & & Jaundiced (yes) & \\
\hline 48 & & Orange & & & & Yes or no \\
\hline
\end{tabular}

Note: The correct response for each trial is located within brackets. 


\section{Appendix I: First Recall Sheet}

ID\#:

\section{FR Sheet (038901)}

This is a surprise memory test. Please write down all words you can recall that you made judgments aboutin the first task. These words were the ones that appeared after you were shown the questions: "Two or more e's?", "Rhymes with XXXX?", "Means the same as XXXX?", and "Describes me?".

You have 5 minutes to recall as many words as possible. Please list recalled words starting on the line below labelled " 1 ." and continuing to higher numbered lines. Every minute, the researcher will call out "Now" which will prompt you to put a SLASH "/" beside the number of the line where you wrote your last recalled word. Please tell the researcher when you are rea dy to start.

1.

2.

3.

4.

5.

6.

7.

8.
9.

10.

11.

12.

13.

14.

15.

16.
17.

18.

19.

20.

21.

22.

23.

24. 


\section{Appendix J: Second Recall Sheet}

$\mathrm{ID} \#$ :

\section{FR Sheet (904582)}

For the second time, please write down all words you can recall from the judgment task. We would like you to try to recall the words you listed the first time, as well as new ones. Like before, list recalled words starting on the line below labelled "1." and continuing to higher numbered lines.

You have 5 minutes for this task. Every minute, the research will call out "Now" which will prompt you to put a $S L A S H$ "/" beside the number of the line where you wrote the last word you recalled. Please tell the researcher when you are ready to start.

1.

2.

3.

4.

5.

6.

7.

8.

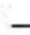

9.

10.

11.

12.

13.

14.

15.

16.
24.

17.

18.

19.

20.

21.

22.

23. 
Appendix K: Zero-Order Correlations Among the Memory-Based, Direct, and Indirect Personality Measures

Table K1

Zero-Order Correlations Among the Direct, Indirect, and Self-Reference Memory Measures (Including Openness)

\begin{tabular}{|c|c|c|c|c|c|c|c|c|c|c|}
\hline Measure & 1 & 2 & 3 & 4 & 5 & 6 & 7 & 8 & $9^{\mathrm{a}}$ & $10^{\mathrm{a}}$ \\
\hline 1. Conscientiousness & - & & & & & & & & & \\
\hline 2. Orderliness & $.65^{* * *}$ & - & & & & & & & & \\
\hline 3. Encoding-Orderliness & $.45^{* * *}$ & $.42^{* * *}$ & - & & & & & & & \\
\hline 4. Orderliness Recall & .11 & .02 & .05 & - & & & & & & \\
\hline 5. Rate of Approach & $-.22^{*}$ & $-.27^{* *}$ & $-.14^{+}$ & $-.31^{* *}$ & - & & & & & \\
\hline 6. Correct Recognition & .06 & -.05 & .03 & $.42^{* * *}$ & -.01 & - & & & & \\
\hline 7. False Recognition & $-.15^{++}$ & -.11 & -.13 & -.05 & .10 & .11 & - & & & \\
\hline 8. Orderliness-IAT & $.18^{+}$ & $.15^{++}$ & $.21^{*}$ & .00 & -.04 & $-.24^{*}$ & $-.17^{++}$ & - & & \\
\hline 9. Inverse Item gain ${ }^{a}$ & -.08 & -.06 & .06 & $-.39^{* * *}$ & $.58^{* * *}$ & -.07 & -.04 & .05 & - & \\
\hline 10. Inverse Item loss ${ }^{a}$ & .06 & .04 & .09 & $.33^{* * *}$ & -.02 & .06 & .00 & -.04 & $-.17^{++}$ & - \\
\hline 11. Openness & .12 & .06 & .09 & $.31^{* *}$ & -.12 & $.19^{+}$ & .08 & $-.16^{++}$ & $-.16^{++}$ & .01 \\
\hline
\end{tabular}

Note. $d f \mathrm{~s}=87-95 .^{\text {a }}$ Inverse transformed. Must be interpreted accordingly.

${ }^{++} p<.20 .{ }^{+} p<.10 .{ }^{*} p<.05 .{ }^{* *} p<.01 .{ }^{* * *} p<.001$. 


\section{Appendix L: Memory List and Recognition Items for Study 2.}

De Raad and Peabody (2005), Goldberg (1990), and thesaurus.com served as the source for imaginativeness adjectives. All hyphenated adjectives and compound adjectives were excluded, as those would provide a confounding cue for retrieval as well as alternative processing strategies (i.e., relational processing based on superficial aspects of the stimuli, e.g., hyphenated versus unhyphenated words). The 13 adjectives that remained from De Raad and Peabody's (2005) imaginativeness facet of intellect were included, and at face value were considered the best indicators of imaginativeness to be used in the present study. However, to increase the pool of available adjectives, Goldberg's (1990) 35 adjectives describing the facets of intellect, 9 unique synonyms and 6 unique antonyms of the word imaginative, and 10 unique synonyms and 8 unique antonyms of the word fanciful from thesaurus.com were included in the master list. In total, there were 81 adjectives. The goal was to reduce this collection of adjectives to 10 that best described the positive pole of imaginativeness and 10 that best described the negative pole. Another objective was to equate each pole of the imaginativeness adjectives and orderliness items used in Study 1 for word frequency, word length, likeability, and meaningfulness. In addition, careful attention was paid to maximize the difference in latent semantic association between the imaginativeness and orderliness concepts between lists.

Word length and word frequency (measured in $\log 10$ [raw frequency count +1$]$ units) were computed following Brysbaert and New (2009) on the Ghent University website (http://expsy.ugent.be/subtlexus/). All adjectives with a relatively low frequency (less than 1.50) were immediately excluded, except for those on the negative pole of imaginativeness because of the limited number to work with. To acquire the four lists of 
10 adjectives exactly, two steps were taken. First, using a many-to-one latent semantic analysis tool on the University of Colorado website (http://sa.colorado.edu/) with a topic space of "General Reading up to 1st year college (300 factors)", the latent semantic associations of the terms imaginative, imagination, fanciful, and creative, as well as order, orderly, and orderliness were computed for all adjectives. Then the average association for each adjective with its corresponding terms (e.g., poetic with imaginative, imagination, fanciful, and creative) was computed as well as the average for the other terms (e.g. poetic with order, orderly, and orderliness). Second, a difference score was created from the average LSA for imaginativeness and the average LSA for orderliness. This difference score served as an index of the separation of the terms in semantic space, with higher values indicating greater separation.

All adjectives were sorted by the size of the difference score, then by the size of the imaginativeness LSA value. The 10 adjectives for the positive pole of imaginativeness presented in Table 7 were selected from those with the highest difference score and LSA value for imaginativeness, with a bias toward selecting adjectives listed in De Raad and Peabody (2005). Also, some of the strongest imaginative terms were not selected for the positive list, because their negated variant (e.g., unromantic for romantic) was required for the negative pole. The 10 adjectives for the negative pole of imaginativeness were selected in the same manner. The orderly and disorderly list from Study 1 were used, except "meticulous" was exchanged for "careful" and the disorderly adjectives "chaotic" and "contradictory" were exchanged for "rash" and "impulsive" because of their strong relation to the imaginativeness concept. Recognition lures were selected following the same procedure. Table L1 presents the recognition lures and Table 
L2 presents the average psycholinguistic properties for each list. The 20 categorically unrelated items that carried the lowest latent semantic association to the trait adjective concepts were selected from the pool developed in Study 1.

Table L1

Recognition Memory Lures for the Imaginative, Orderly, Unimaginative, and Disorderly Lists

\begin{tabular}{cccc}
\hline & \multicolumn{2}{c}{ Recognition Lures } & \\
Imaginative & Orderly & Unimaginative & Disorderly \\
\hline Vivid & Systematic & Literal & Untidy \\
Intellectual & Reliable & Dull & Irresponsible \\
Ingenious & Thorough & Simple & Inconsistent \\
Inquisitive & Businesslike & Ignorant & Lazy \\
Philosophical & Dependable & Naive & Sluggish \\
Perceptive & Practical & Uneducated & Insatiable \\
Gifted & Prudent & Unsophisticated & Neglectful \\
Absurd & Serious & Colorless & Hasty \\
Speculative & Rigorous & Disinterested & Reckless \\
Innovative & Diligent & Ordinary & Unconscientious \\
\hline
\end{tabular}

Table L2

Mean Psycholinguistic Properties for the Imaginativeness and Orderliness Recognition Lures

\begin{tabular}{cccccc}
\hline & Imaginative & Orderly & $\begin{array}{c}\text { Unimaginativ } \\
\mathrm{e}\end{array}$ & Disorderly & \\
\hline Word & $2.00(.44)$ & $2.12(.79)$ & $2.12(.75)$ & $1.94(.54)$ & .19 \\
$\begin{array}{c}\text { Frequency } \\
\text { Word Length }\end{array}$ & $9.30(2.75)$ & $8.70(1.57)$ & $8.50(3.44)$ & $9.10(3.57)$ & .15 \\
\hline
\end{tabular}




\begin{tabular}{cccccc}
\hline Likeableness $^{\mathrm{a}}$ & $423.80^{\mathrm{b}}$ & $430.75^{\mathrm{c}}$ & $231.00^{\mathrm{d}}$ & $156.17^{\mathrm{e}}$ & $28.26^{* * *}$ \\
& $(72.15)$ & $(70.35)$ & $(73.35)$ & $(33.53)$ & \\
Meaningfulness & $352.80^{\mathrm{b}}$ & $358.00^{\mathrm{c}}$ & $344.00^{\mathrm{d}}$ & $371.33^{\mathrm{e}}$ & 1.98 \\
$\mathrm{a}$ & $(22.43)$ & $(21.11)$ & $(14.24)$ & $(11.08)$ & \\
Imaginative & $.26(.09)$ & $.12(.05)$ & $.17(.11)$ & $.10(.03)$ & $9.00^{* * *}$ \\
LSA & & & & & \\
Orderliness & $.11(.06)$ & $.20(.08)$ & $.10(.06)$ & $.09(.04)$ & $6.04^{* *}$ \\
LSA & & & & & \\
\hline
\end{tabular}

Note. Standard deviation is presented within parentheses.

${ }^{a}$ Calculated from Anderson's (1968) personality trait norms. ${ }^{b}$ missing data for vivid, gifted, absurd, speculative, and innovative. ${ }^{\mathrm{c}}$ missing data for businesslike and rigorous ${ }^{\mathrm{d}}$ missing data for literal, ignorant, simple, uneducated, colorless, and disinterested ${ }^{\mathrm{e}}$ missing data for sluggish, hasty, insatiable, and unconsientious.

${ }^{* *} p<.01{ }^{* * * *} p<.001$. 


\section{Appendix M: Friend Questionnaire.}

ID\#:

\section{Friend Questionnaire}

Periodically throughout this experiment, you will engage in tasks that require you to have a close friend in mind (e.g., a best friend, family member, classmate, etc).

Right now, please take a minute to decide who this individual is.

Every time in the experiment when you are asked about your "friend", please refer to this specific individual. All responses will be kept in absolute confidence.

Please take a minute to answer the questions below.

1. What is your friend's first name?

2. How frequently do you currently communicate with your friend?

$\square$ 1 Daily

$\square_{2}$ Weekly

$\square_{3}$ Monthly

$\square_{4}$ Yearly

3. How many years have you known your friend?

4. Please indicate how well you know your friend using the following rating scale (circle your response):

$\begin{array}{ccccccc}1 & 2 & 3 & 4 & 5 & 6 & 7 \\ \text { Not Well } & & & \begin{array}{c}\text { Moderately } \\ \text { Well }\end{array} & & & \\ & & & & & & \text { Extremely } \\ & & & & \text { Well }\end{array}$

5. Please circle the picture that best describes how close you and your friend are.
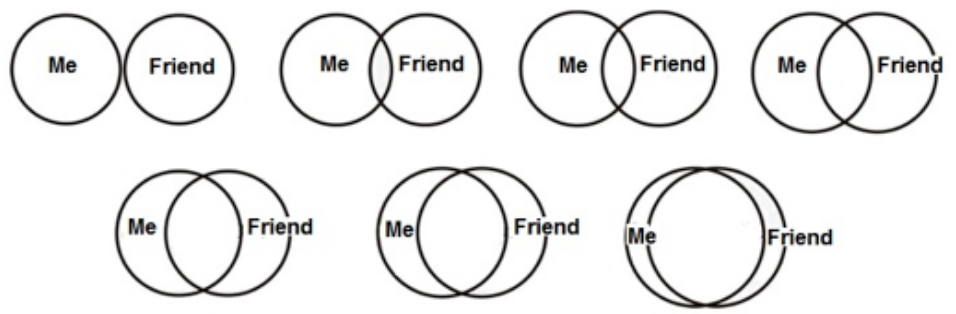


\section{Appendix N: Recognition Items for Study 3.}

Table N1

Recognition Memory Lures for the Trust, Orderly, and Imaginative Lists.

\begin{tabular}{ccc}
\hline Trust & Recognition Lures & \\
Loving & Orderly & Imaginative \\
Genuine & Businesslike & Vivid \\
Affectionate & Dependable & Intellectual \\
Unassuming & Practical & Ingenious \\
Sincere & Prudent & Inquisitive \\
Polite & Serious & Philosophical \\
Considerate & Rigorous & Perceptive \\
Respectful & Diligent & Absurd \\
Forgiving & Provident & Speculative \\
Tender & Severe & Innovative \\
Sensitive & Productive & Stimulating \\
Truthful & Alert & Interested \\
Humble & Competent & Analytical \\
& Reasonable & Brilliant
\end{tabular}

Table N2

Mean Psycholinguistic Properties for the Imaginativeness and Orderliness Recognition Lures

\begin{tabular}{ccccc}
\hline & Trust & Orderly & Imaginative & $F$ \\
\hline Word & $2.16(.69)$ & $2.17(.89)$ & $2.40(.71)$ & .46 \\
Frequency & & & & \\
Word Length & $9.77(2.20)$ & $8.46(1.90)$ & $8.23(2.09)$ & 2.09 \\
& $434.83^{\mathrm{b}}$ & $448.00^{\mathrm{c}}$ & $482.67^{\mathrm{d}}$ & \\
Likeableness $^{\mathrm{a}}$ & $(69.96)$ & $(58.27)$ & $(64.63)$ & 1.18 \\
\hline
\end{tabular}




\begin{tabular}{ccccc}
\hline $\begin{array}{c}\text { Meaningfulness } \\
\mathrm{a}\end{array}$ & $\begin{array}{c}355.00^{\mathrm{b}} \\
(20.77)\end{array}$ & $\begin{array}{c}362.00^{\mathrm{c}} \\
(18.79)\end{array}$ & $\begin{array}{c}365.56^{\mathrm{d}} \\
(13.48)\end{array}$ & .66 \\
$\begin{array}{c}\text { Imaginative } \\
\text { LSA }\end{array}$ & $.24(.09)$ & $.11(.05)$ & $.12(.06)$ & $14.73^{* * *}$ \\
Orderliness & $.12(.05)$ & $.16(.06)$ & $.09(.04)$ & $7.15^{* *}$ \\
LSA & $.11(.06)$ & $.11(.06)$ & $.18(.07)$ & $5.51^{* *}$ \\
Trust LSA & & & & \\
\hline
\end{tabular}

Note. Standard deviation is presented within parentheses.

${ }^{a}$ Calculated from Anderson's (1968) personality trait norms. ${ }^{b}$ loving, genuine, affectionate, and unassuming ${ }^{c}$ missing data for businesslike, rigorous, provident, and severe ${ }^{\mathrm{d}}$ missing data for vivid, innovative, absurd, speculative, interested, analytical, and stimulating.

${ }^{* *} p<.01{ }^{* * *} p<.001$. 